\title{
Physical and Digital Security
}

Mechanisms:

\section{and Trade-offs}

\section{André van Cleeff}

0000000 
Physical and Digital Security Mechanisms:

Properties, Combinations and Trade-offs

André van Cleeff 
Composition of the Graduation Committee:

Prof. dr. P.M.G. Apers

Prof. dr. R.J. Wieringa

Dr. W. Pieters

Prof. dr. P.H. Hartel

Prof. dr. J. van Hillegersberg

Prof. dr. E. Dubois

Dr. L. Coles-Kemp

Prof. dr-Eng. F. Massacci
Universiteit Twente (Chairman and Secretary)

Universiteit Twente (Promotor)

TU Delft (Assistant Promotor)

Universiteit Twente

Universiteit Twente

CRP Henri Tudor / University of Luxembourg

Royal Holloway (University of London)

Universita di Trento

Services, Cybersecurity and Safety

Research Group

UNIVERSITY OF TWENTE. P.O. Box 217, 7500 AE Enschede

The Netherlands

This research is supported by the research

SENTİNELS program Sentinels of STW

(http://www.sentinels.nl)

under the project number 06679.

CTIT PhD Thesis Series Number 15-360

CTIT

Centre for Telematics and Information

Technology (CTIT)

P.O. Box 217, 7500 AE Enschede

The Netherlands

SIKS Dissertation Series No. 2015-17

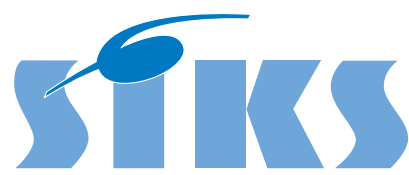

The research reported in this thesis has been carried out under the auspices of SIKS, the Dutch Research School for Information and Knowledge Systems.

ISBN: 978-90-365-3884-8

ISSN: $1381-3617$

DOI: http://dx.doi.org/10.3990/1.9789036538848

Printed by Ipskamp Drukkers B.V. the Netherlands

Copyright (C) 2015 André van Cleeff 


\section{PHYSICAL AND DIGITAL SECURITY MECHANISMS: PROPERTIES, COMBINATIONS AND TRADE-OFFS}

\section{DISSERTATION}

to obtain

the degree of doctor at the University of Twente on the authority of the rector magnificus, Prof. Dr. H. Brinksma, on account of the decision of the graduation committee, to be publicly defended on Wednesday, 3rd of June, 2015 at 12:45

by

André van Cleeff

born on 3rd of July 1977

in Rotterdam, the Netherlands 
The dissertation is approved by:

Prof. dr. R.J. Wieringa Universiteit Twente (Promotor)

Dr. W. Pieters

TU Delft (Assistant Promotor) 
To Haiyan \& Louisa 


\section{Abstract}

The usage of information technology implies the replacement of physical systems with digital systems: we use information technology because some properties of software, such as high speed, low cost and high accuracy, are more desirable than the corresponding properties of physical systems.

Unfortunately, digital systems are not uniformly more secure than physical systems and automation can have a negative effect on the confidentiality, integrity and availability of information. Specifically, the Internet helps to spread information, which makes it harder to keep it confidential. The increased connectivity caused by the Internet makes organizations become "de-perimeterized": the physical barriers that once separated them are breaking down.

We observe however that there is no extensive and structured body of knowledge on the differences between physical and digital systems and the way that de-perimeterization takes place. Obtaining this knowledge becomes more important now that physical and digital systems are merging in the Internet of Things: only when we understand the differences between physical and digital systems can we truly design secure combinations such as smart buildings with cameras and door locks operable by mobile phone.

Developing this knowledge starts with a simple conceptual framework: systems range from being completely physical to completely automated. The former only use physical security mechanisms, whereas the latter only use digital security mechanisms. In between these lies a mixed category of hybrid systems, which can use both digital and physical security mechanisms. Following this framework we study the security of physical, digital and hybrid systems in four domains: access control, voting in elections, IT infrastructure and rights management. We begin with investigating the underlying properties of physical and digital systems: characteristics of a physical or digital object that, under specific conditions, have positive or negative effects on security. In total we present twenty physical and five digital security properties. These properties are then used to identify the differences between physical and digital security in each case. Next we examine 
hybrid systems to understand how to combine physical and digital security, and what the trade-offs are between these two. Finally, these results are used to create two methods that help improve information security:

- A method for assessing security risks of physical, digital and hybrid systems. This method is built around security properties: they are used to understand the security of existing systems (by identifying the properties and how they could change) or to design new systems (by building in those properties and conditions that have positive effects on security).

- A method for assessing the security of hybrid systems through security patterns. These patterns are reusable designs that show how to combine physical and digital security optimally. We present a total of thirteen patterns that are useful both to design and to evaluate the security of hybrid systems.

Both methods were tested successfully in a focus group meeting with security experts. 


\section{Acknowledgements}

"I want to learn how to do good research." These words slipped out of my mouth many years ago in a job interview. I was not hired, and had to wait almost ten years until I got another opportunity at the University of Twente. I feel very fortunate that I was allowed to work on achieving that goal. Not only that, the past years were a wonderful time that I spend teaching, meeting lots of new people and travelling around the globe.

So, some acknowledgements are in order. First of all, I want to go back in time a bit and appreciate the hard work that went into getting funds for the VISPER project. Pascal, Pieter and Roel: without your efforts I would not have been able to come to Twente in the first place. I am very pleased that we managed to get the PISA proposal accepted and the research can continue!

Specifically, I must acknowledge the efforts of my promotor, Roel Wieringa. You always had new ideas and after most of the monthly meetings I walked back with a more clear idea about what I could do next. Furthermore, who else can explain you about Italian finance in the 14th century, and actually use it to clarify the problem at hand?

Thanks also goes out to Wolter, who as the VISPER Postdoc commented on my drafts and helped to structure my thesis. I also greatly appreciated the CPDP workshop in Brussels and Dagstuhl seminar that we organized. Furthermore, I also want to thank Sjouke Mauw for inviting me to give a talk at Luxembourg while I was finishing my thesis. If not for that trip, Figure 1.1 would not have been there. Also the committee members, thank you for taking the time to review my thesis and giving me suggestions for improving parts of it. The implementation of these took a bit more time than expected but the final result certainly improved. Finally I express my appreciation to the case study partners, which I cannot mention here as this would breach the NDA $\odot$.

Next, the research group that I was part of (IS) and that I worked with (DIES) and which is now called SCS: Brahmananda, Chen, Eelco, Emmanuele, Hassan, Jelena, Lianne, Maya, Mohammed, Shahin, Silja, Suse, Trajce, Virginia, Wilco, 
Zornitza. Arjan, Ayse, Begl, Bertine, Christoph, Damiano, Dina, Emmanuele, Frank, Giorgi, Jonathan, Marcin, Michael, Michele, Mohammed, Mohsen, Nienke, Qiang, Richard, Saeed, Stefan, Svetla. I really had wonderful colleagues and to be honest, I actually enjoyed going to our weekly seminar meetings. Thanks for the nice coffee breaks, group outings and the sports event. I have especially happy memories of our trip to Giethoorn. Also it's great that we managed to continue the Sinterklaas tradition up to this date, even though each of us moved so many times in between!

Now the friends I met in Enschede: "TJ" Dimkov, you introduced me to Macandra, we went to Macedonia, to Ibiza and to the gym. All those things were exhausting in their own way, but I would not like to have missed them. Ove $\&$ Shashank, I don't have that much hope for your Dutch now that you left (the) Netherlands, but the four of us can still try to continue the yearly trip to Maarn, of course together with Paula, Lena and Anne. Paris and Frankfurt are not that far away! Esly, thanks for having the energy for organizing so many events and parties. Nima, Katja, Somayeh, Amir, Faiza, Faizan, Ram \& Saba, let's finish that tour around the provinces!

My parents: now that I have became a parent myself, I appreciate more and more the effort that you put in raising and educating me. As for the $\mathrm{PhD}, \mathrm{I}$ am thankful for your support during these years in Enschede, and for your efforts to visit me there and invite me to explore beautiful museums and churches. Annette, Jules, Anne, Theo, thanks for all the help!

Finally of course Haiyan and Louisa. Haiyan, I am so happy we both came to Enschede and met each other. Finishing both of our theses seemed "a bridge too far" at times, but we did pull it off in the end. I am very lucky to have you in my life, thank you for all the love \& support during the past years! Louisa, your birth inspired me to finish the thesis, and soon you can call your father a doctor (). Thanks for being with us! Haiyan and I promise not to be tiger parents, but we are certainly very curious to find out how much study drive you have inherited from us $\odot$.

So dear reader, the result of many years of work is in your hands. I don't expect you to read it all, so I put a lot of effort in my abstract... Hopefully you will appreciate it!

De Meern, May 2015. 


\section{Contents}

I Problem Investigation and Research Design 1

$\begin{array}{lll}1 & \text { Introduction } & 3\end{array}$

1.1 Research Motivation . . . . . . . . . . . . . . . . . . 4

1.2 Research Questions . . . . . . . . . . . . . . . . . . . . 5

1.3 Research Scope . . . . . . . . . . . . . . . . . . . . . . 6

1.4 Research Design and Methods . . . . . . . . . . . . . . . 7

1.5 Contribution to Knowledge $\ldots \ldots \ldots \ldots$

1.6 Implications for Practice $\ldots \ldots \ldots$

1.7 Outline of the Dissertation . . . . . . . . . . . . . . . . . 10

1.8 Summary . . . . . . . . . . . . . . . . . . 10

\begin{tabular}{|lll}
\hline 2 & Terminology & 13
\end{tabular}

$2.1 \quad$ The Physical and the Digital World . . . . . . . . . . . . . . 13

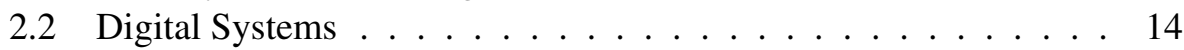

2.3 Information Security $\ldots \ldots \ldots \ldots \ldots \ldots$

\begin{tabular}{llr}
\hline 3 & Historical Background & 19
\end{tabular}

3.1 Introduction . . . . . . . . . . . . . . . . . . . . . . 19

3.2 De-perimeterization . . . . . . . . . . . . . . . . . . . 20

3.3 Analysis of De-perimeterization . . . . . . . . . . . . 22

3.4 Treatment of De-perimeterization . . . . . . . . . . . . . 25

3.5 Conclusion $\ldots \ldots \ldots \ldots \ldots \ldots \ldots \ldots \ldots$

\begin{tabular}{|lll}
\hline & Related Work & 37
\end{tabular}

4.1 Broad-Range Theories . . . . . . . . . . . . . . . . . . . . 37

4.2 Integrated Security Mechanisms . . . . . . . . . . . . . . . . . . 39

4.3 IT Architecture Frameworks . . . . . . . . . . . . . . . . . 40

4.4 Formal Models and Simulations $\ldots \ldots \ldots$. . . . . . . . . 41

4.5 Summary . . . . . . . . . . . . . . . . . 42 
5 Method 45

5.1 Introduction . . . . . . . . . . . . . . . . . . . . 45

5.2 Research Design $\ldots \ldots \ldots$. . . . . . . . . . . . . 45

5.3 Case Selection . . . . . . . . . . . . . . . . . . . . 46

5.4 System Representation . . . . . . . . . . . . . . . . . . . . . . . 49

5.5 System Data Collection . . . . . . . . . . . . . . . . . . . 53

$5.6 \quad$ Outline for Each Case Study $\ldots \ldots \ldots$. . . . . . . . . . . 54

5.7 Analysis of Multiple Cases $\ldots \ldots \ldots \ldots$

5.8 Properties $\ldots \ldots \ldots \ldots \ldots \ldots \ldots \ldots \ldots \ldots$

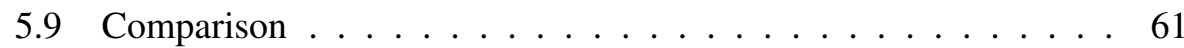

5.10 Combinations . . . . . . . . . . . . . . . 62

5.11 Trade-off Analysis . . . . . . . . . . . . . . . . . 62

5.12 Application of Results . . . . . . . . . . . . . . . . . . 64

5.13 Validity $\ldots \ldots \ldots \ldots \ldots \ldots$

II Case Studies 69

\begin{tabular}{|lll}
6 & Access Control Systems & 71
\end{tabular}

6.1 Introduction $\ldots \ldots \ldots \ldots \ldots \ldots \ldots \ldots$

6.2 Conceptual Framework $\ldots \ldots \ldots$. . . . . . . . . . . 73

6.3 Method . . . . . . . . . . . . . . . . 76

6.4 Goals . . . . . . . . . . . . . . . . . . . . . . . . . . 79

6.5 Physical Access Control _. . . . . . . . . . . . . . . . . 82

6.6 Properties of Physical Entities $\ldots \ldots \ldots \ldots$

6.7 Logical Access Control . . . . . . . . . . . . . . . . . . . . . . . 89

6.8 Properties of Digital Entities $\ldots \ldots \ldots$

6.9 Comparison between Physical and Logical Access Control . . . . 91

6.10 Location-Based Access Control . . . . . . . . . . . . . . . 96

6.11 Trade-off Analysis ． . . . . . . . . . . . . . . . . . . . . 100

6.12 Conclusion . . . . . . . . . . . . . . . . . . 106

\begin{tabular}{lll}
\hline 7 & Voting Systems & 109
\end{tabular}

7.1 Introduction . . . . . . . . . . . . . . . . . . . . . . . . . 109

7.2 Conceptual Framework $\ldots \ldots \ldots$

7.3 Method . . . . . . . . . . . . . . . . 113

7.4 Goals . . . . . . . . . . . . . . . . . . . . 115

7.5 Paper Voting . . . . . . . . . . . . . . . . . . . . . 118

7.6 Properties of Physical Entities $\ldots \ldots \ldots$

7.7 Electronic Voting . . . . . . . . . . . . . . . . . . . . . 127

7.8 Properties of Digital Entities $\ldots \ldots$. . . . . . . . . . . . 132

7.9 Comparison between Paper and Electronic Voting . . . . . . . . . 134 
7.10 Hybrid Voting . . . . . . . . . . . . . . . . . . . . 137

7.11 Combinations and Trade-off Analysis . . . . . . . . . . . . 140

7.12 Conclusion . . . . . . . . . . . . . . . . . 144

$\begin{array}{lll}8 & \text { IT Infrastructure } & 147\end{array}$

8.1 Introduction . . . . . . . . . . . . . . . . . . . . 147

8.2 Conceptual Framework . . . . . . . . . . . . . . . . . . . . . . 148

8.3 Method . . . . . . . . . . . . . . . . . . . . . . . . . 149

8.4 Goals . . . . . . . . . . . . . . . . . . . . . . . . . 154

8.5 Physical IT Infrastructure . . . . . . . . . . . . . . . 156

8.6 Properties of Physical Entities $\ldots \ldots$. . . . . . . . . . . . 157

$8.7 \quad$ Virtualized IT Infrastructure $\ldots \ldots \ldots \ldots$

$8.8 \quad$ Properties of Digital Entities $\ldots \ldots$. . . . . . . . . . 170

8.9 Comparison between Physical and Virtualized IT Infrastructure. . 173

8.10 Hybrid IT Infrastructure . . . . . . . . . . . . . . . 177

8.11 Combinations and Trade-off Analysis . . . . . . . . . . . . 179

8.12 Conclusion . . . . . . . . . . . . . . . . . 182

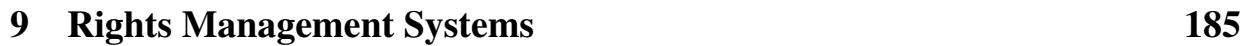

9.1 Introduction . . . . . . . . . . . . . . . . . . . 185

9.2 Conceptual Framework ． . . . . . . . . . . . . . . . . . 186

9.3 Method . . . . . . . . . . . . . . . . . . . . . . . 191

9.4 Goals . . . . . . . . . . . . . . . . . . . . . . . . . . . 193

9.5 Physical Rights Management $\ldots \ldots \ldots$. . . . . . . . . . . . . . . 194

9.6 Properties of Physical Entities … . . . . . . . . . . . . . 197

9.7 Digital Rights Management. . . . . . . . . . . . . . . . . . . . . 199

9.8 Properties of Digital Entities . . . . . . . . . . . . . . . . . 201

9.9 Comparison between Physical and Digital Rights Management . . 203

9.10 Hybrid Rights Management . . . . . . . . . . . . . . . . . 206

9.11 Combinations and Trade-off Analysis . . . . . . . . . . . . 210

9.12 Conclusion . . . . . . . . . . . . . . . . . 213

\begin{tabular}{lll}
\hline III Summary, Application and Validation & 217
\end{tabular}

10 Conceptual Model of Integrated Physical and Digital Security 219

10.1 Introduction . . . . . . . . . . . . . . . . . 219

10.2 Method . . . . . . . . . . . . . . . . . . . 220

10.3 Properties of Physical Entities . . . . . . . . . . . . . . 220

10.4 Properties of Digital Entities . . . . . . . . . . . . . 226

10.5 Comparison between Physical and Digital Systems . . . . . . . 228

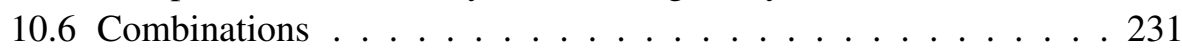


10.7 Trade-off Analysis _ . . . . . . . . . . . . . . . . 235

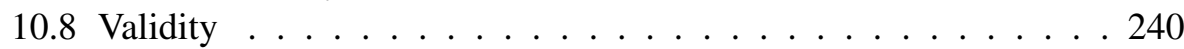

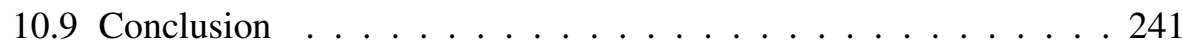

11 Application of Results 245

11.1 Introduction . . . . . . . . . . . . . . . . . . . 245

11.2 Application per Research Question . . . . . . . . . . . . . . 245

11.3 Application in Case Studies . . . . . . . . . . . . . . . . . . . . 248

11.4 Application in Risk Assessment and System Design . . . . . . . . 250

12 Validation in Focus Group Meeting 261

12.1 Introduction . . . . . . . . . . . . . . . . . . 261

12.2 Method . . . . . . . . . . . . . . . . . . 262

12.3 Results . . . . . . . . . . . . . . . . . . . . 265

12.4 Discussion and Conclusions $\ldots \ldots \ldots$. . . . . . . . 273

13 Conclusions 277

13.1 Introduction . . . . . . . . . . . . . . . . . . . . 277

13.2 Summary of Findings . . . . . . . . . . . . . . . . 277

13.3 Reflection . . . . . . . . . . . . . . . . . . . . 278

13.4 Outlook . . . . . . . . . . . . . . . . . . . 282

13.5 Future Work . . . . . . . . . . . . . . . . . . . . . 283

13.6 Conclusion . . . . . . . . . . . . . . . . . 285

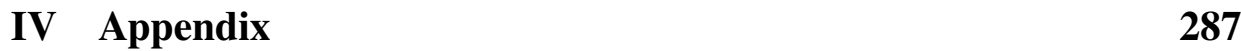

\begin{tabular}{|ll}
\hline A Focus Group Meeting Material & 289
\end{tabular}

A.1 Questionnaire . . . . . . . . . . . . . . . . . . . . . . . . 290

A.2 Linkage between Focus Group Questions and Research Questions 298

A.3 Answers for Method One . . . . . . . . . . . . . . . . . . . . 298

A.4 Integrated Security Patterns . . . . . . . . . . . . . . . . 304

\begin{tabular}{ll}
\hline Bibliography & 309
\end{tabular}

\begin{tabular}{ll}
\hline SIKS Dissertation Series & 331
\end{tabular}

\begin{tabular}{lr}
\hline Samenvatting & 359
\end{tabular} 


\section{Acronyms}

ABE Attribute-Based Encryption.

CIA Confidentiality, Integrity, Availability.

COA Collaboration-Oriented Architecture.

CPS Cyber-Physical System.

CPU Central Processing Unit.

DAT Digital Audio Tape.

DLP Data Loss Prevention.

DOS Denial-Of-Service.

DRE Direct-Recording Electronic.

DRM Digital Rights Management.

HSM Hardware Security Module.

IP Intellectual Property.

ISP Internet Service Provider.

LAC Logical Access Control.

LBAC Location-Based Access Control.

MAC Mandatory Access Control. 
PAC Physical Access Control.

PAP Policy Administration Point.

PDP Policy Decision Point.

PEP Policy Enforcement Point.

PIP Policy Information Point.

PRM Physical Rights Management.

RBAC Role-Based Access Control.

SAN Storage Array Network.

SoD Separation of Duty.

TPM Trusted Platform Module.

UCON Usage Control.

VM Virtual Machine.

VMM Virtual Machine Monitor.

VMMM Virtual Machine Monitors' Management.

VPN Virtual Private Network.

VVPAT Voter-Verified Paper Audit Trail.

XACML eXtensible Access Control Markup Language. 


\section{List of Figures}

$\begin{array}{lll}1.1 & \text { Simple conceptual framework about physical and digital systems. } 5\end{array}$

1.2 Research design with layered case studies. . . . . . . . . . . . . . . 9

1.3 Outline of the thesis. . . . . . . . . . . . . . . 10

$2.1 \quad$ The relations between the physical and digital domain. . . . . . . 14

3.1 The Jericho Forum's view on de-perimeterization. . . . . . . . . . 21

3.2 Alternative view on de-perimeterization. . . . . . . . . . . . . . . 24

3.3 Jericho Forum Commandments . . . . . . . . . . . . . . . . . 25

$5.1 \quad$ Research design with layered case studies. . . . . . . . . . . . . 47

$5.2 \quad$ List of case studies. . . . . . . . . . . . . . . . . . 48

5.3 Schematic overview of a Toulmin argument. . . . . . . . . 50

5.4 KAOS terminology with explanations. . . . . . . . . . . . 52

5.5 Outline for each case study. . . . . . . . . . . . . . . . . 54

$5.6 \quad$ Parts used in constructing the KAOS tree. . . . . . . . . . . . 55

$5.7 \quad$ Example KAOS tree, showing individual parts of its construction. 55

$5.8 \quad$ Finding related model elements for an entity. $\ldots \ldots \ldots . . . .58$

5.9 Example comparison of physical and digital systems. . . . . . . . 62

5.10 Example comparison of hybrid with physical and digital systems. 63

5.11 Assessment scores of comparing hybrid with physical and digital

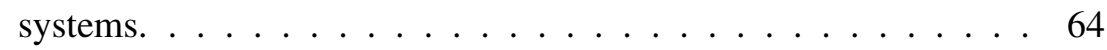

5.12 Elements of a design pattern. . . . . . . . . . . . . 65

$6.1 \quad$ Security mechanisms in different domains. . . . . . . . . . . . 71

6.2 A typical reference monitor. . . . . . . . . . . . . 73

6.3 UCON dimensions. . . . . . . . . . . . . . . . . . . . . . . . 74

$6.4 \quad$ Search terms used in the literature study. . . . . . . . . . . . . 78

$6.5 \quad$ Goals of access control. . . . . . . . . . . . . . . . . 80 
6.6 Typical layout of a facility. . . . . . . . . . . . . . . 82

6.7 Entities used in physical access control. . . . . . . . . . . 83

6.8 Operations used in physical access control. . . . . . . . . . . . 84

6.9 Threats to physical access control goals. . . . . . . . . . . . . . 84

$6.10 \quad$ New hypotheses from the access control case study about the security effects of physical properties of entities. . . . . . . . . 86

6.11 The RBAC model. . . . . . . . . . . . . . . . . . . . . . . 89

6.12 Entities used for logical access control. . . . . . . . . . . . . 90

6.13 Operations used in logical access control. . . . . . . . . . . . . . 91

6.14 New hypotheses from the access control case study about the security effects of digital properties of entities. . . . . . . . . . . 91

6.15 Comparison of physical and digital properties on their ability to realize security goals for the access control case study. . . . . . 92

6.16 Comparison of maintainability for physical and digital access con-

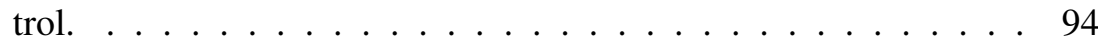

6.17 Threats to location-based access control goals. . . . . . . . . . 99

6.18 Types of hybrid systems in the access control case study. . . . . . 100

6.19 Overview of the four main use cases for LBAC. . . . . . . . . . 102

6.20 Trade-off analysis on physical properties in the access control case study. . . . . . . . . . . . . . . . . 103

6.21 Trade-off analysis on digital properties in the access control case study. . . . . . . . . . . . . . . . . . . 104

6.22 LBAC compared to physical and logical access control on goal

$7.1 \quad$ Top-level goal tree. . . . . . . . . . . . . . . . . . 116

$7.2 \quad$ Entities used in paper voting. . . . . . . . . . . . . . . . . 119

7.3 Operations performed by agents on entities, and their contribution

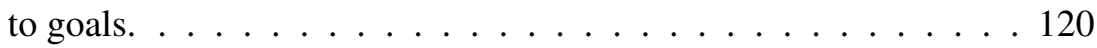

$7.4 \quad$ Threats to voting goals for paper voting. . . . . . . . . . . 122

7.5 Support for existing hypotheses in the voting case study. . . . . . 125

7.6 New hypotheses from the voting case study about the security effects of physical properties of entities. . . . . . . . . . 126

7.7 Additional entities used for electronic voting. . . . . . . . . . 128

7.8 Operations performed by agents and their contribution to goals. . . 129

7.9 Threats to voting goals for electronic voting. . . . . . . . . . . 130

7.10 Support for existing hypotheses in the voting case study. . . . . . 133

7.11 New hypotheses from the voting case study about the security effects of digital properties of entities. . . . . . . . . . . . . 134

7.12 Comparison of physical and digital properties on their ability to realize security goals for the voting case study. . . . . . . . . . 135

7.13 Comparison between physical and digital voting systems. . . . . . 136 
7.14 Threats to voting goals for hybrid voting. . . . . . . . . . . 138

7.15 Types of hybrid systems in the voting case study. . . . . . . . . . 140

7.16 Trade-off analysis on physical properties in the voting case study. .141

7.17 Trade-off analysis on digital properties in the voting case study. . . 142

7.18 Comparison of hybrid voting systems with physical and electronic

systems. . . . . . . . . . . . . . . . . 142

7.19 Trade-off between confidentiality and integrity for voting systems. 145

7.20 Effect of automation on integrity after dropping the confidentiality

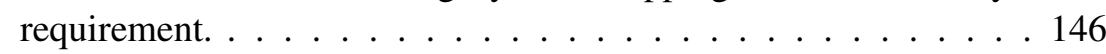

8.1 Virtualization types and corresponding physical and virtualized entities. . . . . . . . . . . . . . . . . 149

8.2 Virtualization entities. . . . . . . . . . . . . . . . 150

8.3 Data sources for sections. . . . . . . . . . . . . . . . . 150

8.4 Interviews for case studies. . . . . . . . . . . . . . . . 153

8.5 Data collected from case studies. . . . . . . . . . . . . . . . 153

8.6 Control categories in relation to COBIT definitions. . . . . . . . 155

$8.7 \quad$ IT Infrastructure goal tree. $\ldots \ldots \ldots \ldots \ldots$

8.8 Selected entities used in physical IT Infrastructures. . . . . . . . . 156

8.9 Physical operations and their contributions to goals. . . . . . . . 157

8.10 Threats to goals for physical IT infrastructure. . . . . . . . . . . 157

8.11 Support for existing hypotheses in the IT infrastructure case study. 159

8.12 New hypotheses from the IT infrastructure case study about the security effects of physical properties of entities. . . . . . . . . . 159

8.13 Overview of virtualization entities. . . . . . . . . . . . . . 160

8.14 Digital operations and their contribution to goals. . . . . . . . . 160

8.15 Virtualization threats. . . . . . . . . . . . . . . . . . 161

8.16 Overview of the impact of virtualization features. . . . . . . . . 163

8.17 Dependencies between virtualization features. . . . . . . . . . . 164

8.18 Support for existing hypotheses in the IT infrastructure case study. 171

8.19 New hypotheses from the IT infrastructure case study about the

security effects of digital properties of entities. . . . . . . . . 172

8.20 Comparison of physical and digital properties on their ability to realize security goals for the IT infrastructure case study. . . . . . 174

8.21 Comparison between physical and virtualized IT infrastructure. . . 175

8.22 Physical and virtual connections. . . . . . . . . . . . . 178

8.23 Types of hybrid systems in the IT infrastructure case study. . . . . 179

8.24 Trade-off analysis on physical properties in the IT infrastructure case study. . . . . . . . . . . . . . . . . . 180

8.25 Trade-off analysis on digital properties in the IT infrastructure

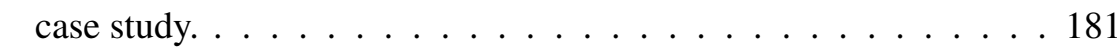

8.26 Comparison of hybrid with physical and virtualized systems. . . . 181 
9.1 Physical, digital and hybrid rights management systems. . . . . 187

9.2 Overview of cases. . . . . . . . . . . . . . . . 191

9.3 Top-level goal tree for rights management. . . . . . . . . . . . . 193

$9.4 \quad$ Entities in physical rights management systems. . . . . . . . . . . 195

9.5 Operations of physical rights management systems and their con-

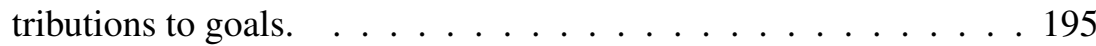

9.6 Threats to physical rights management. . . . . . . . . . . . 196

9.7 Support for existing hypotheses in the rights management case study. . . . . . . . . . . . . . . . . . . 198

9.8 New hypotheses from the rights management case study about the security effects of physical properties of entities. . . . . . . . . 198

9.9 Entities in digital rights management systems. . . . . . . . . . . . 199

9.10 Operations of digital rights management systems and their contributions to goals. . . . . . . . . . . . . . . . . 200

9.11 Threats to digital rights management. . . . . . . . . . . 201

9.12 Support for existing hypotheses in the rights management case

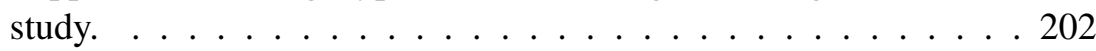

9.13 New hypotheses from the rights management case study about the security effects of digital properties of entities. . . . . . . . . . 202

9.14 Cases for comparison. . . . . . . . . . . . . . . . 203

9.15 Comparison of physical and digital properties on their ability to realize security goals for the rights management case study. . . . . 204

9.16 Comparison between physical and digital rights management sys-

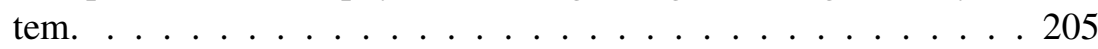

9.17 Entities in hybrid rights management systems. . . . . . . . . . 206

9.18 Operations of hybrid rights management systems and their contributions to goals. . . . . . . . . . . . . . . 207

9.19 Specific mitigations for hybrid rights management. . . . . . . . . 207

9.20 Types of hybrid systems in the rights management case study. . . . 210

9.21 Trade-off analysis on physical properties in the rights management case study. . . . . . . . . . . . . . . . 211

9.22 Trade-off analysis on digital properties in the rights management case study. . . . . . . . . . . . . . . . . . . . 212

9.23 Comparison of hybrid with physical and digital rights management systems. . . . . . . . . . . . . . . . 213

10.1 Complete list of physical properties and their effects. . . . . . . 223

10.2 Clusters of physical properties. . . . . . . . . . . . . . . 224

10.3 Complete list of digital properties and their effects. . . . . . . . . 227

$10.4 \quad$ Clusters of digital properties. $\ldots \ldots \ldots \ldots \ldots$

10.5 Comparison of all physical and digital properties on their ability to realize security goals. . . . . . . . . . . . . . . . . 229 
10.6 Summary of hybrid system types. . . . . . . . . . . . . . 231

20.7 Taxonomy of hybrid systems. . . . . . . . . . . . . . . . . 232

10.8 Explanation of hybrid system taxonomy with applications. . . . . 234

10.9 Trade-off analysis on all physical properties. . . . . . . . . . 236

10.10 Trade-off analysis on all digital properties. . . . . . . . . . . 237

10.11 Hybrid systems compared to physical and digital systems. . . . . 239

10.12 Qualitative representation of trade-offs for realizing security goals in physical, hybrid and digital systems. . . . . . . . . . . . 239

10.13 Newly discovered hypothesis in each subsequent case study. . . . 241

11.1 Schema of a physically configurable switch. . . . . . . . . . . . 248

11.2 Beneficial physical and digital properties of the proposed switch. . 248

11.3 Structure of the integrated risk assessment method. . . . . . . . 250

11.4 Taxonomy of patterns of hybrid systems. . . . . . . . . . . 253

11.5 Elements of a design pattern. . . . . . . . . . . . . . . 254

11.6 Isolated system pattern. . . . . . . . . . . . . . . . . . . . . . . 254

11.7 Location dependent system. . . . . . . . . . . . . . . . . 255

11.8 Person dependent system. . . . . . . . . . . . . . . 255

11.9 Explicit hardware dependent system. . . . . . . . . . . . 256

11.10 Processing power dependent system. . . . . . . . . . . . 256

11.11 Data storage dependent system. . . . . . . . . . . . . . . 257

11.12 Interactivity dependent system. . . . . . . . . . . . . . 257

11.13 Visualization dependent system. . . . . . . . . . . . . . . . 258

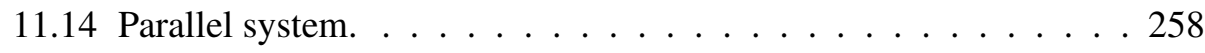

11.15 Serial system. . . . . . . . . . . . . . . . . . . . 259

11.16 Digital monitoring of physical system. . . . . . . . . . . . 259

11.17 Digital validation of physical structure. . . . . . . . . . . . . 260

11.18 Automation of physical process steps. . . . . . . . . . . . 260

12.1 Details of focus group meeting participants. . . . . . . . . . . 266

12.2 Model answers for identifying properties in entities. . . . . . . . . 267

12.3 Correctness percentages for identifying properties. . . . . . . . 268

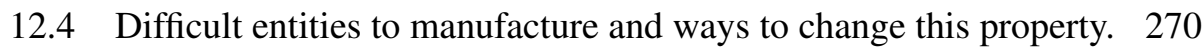

12.5 Impact on security goals when the property difficultly manufacturable changes. . . . . . . . . . . . . . . 2 270

12.6 Results for patterns. . . . . . . . . . . . . . . . 271

13.1 Overview of systems. . . . . . . . . . . . . . . . . . . 279

A.1 Linkage between focus group questions and research questions. . . 298

A.2 Central processing system properties identified by participants. . . 299

A.3 Card reader properties as identified by the participants. . . . . . . 300

A.4 Chip card properties as identified by the participants. . . . . . . . 301 
A.5 Chip properties as identified by the participants. . . . . . . . . . . 302

A.6 Bus, tram or metro properties as identified by the participants. . . 303 


\section{Part I}

\section{Problem Investigation and Research Design}





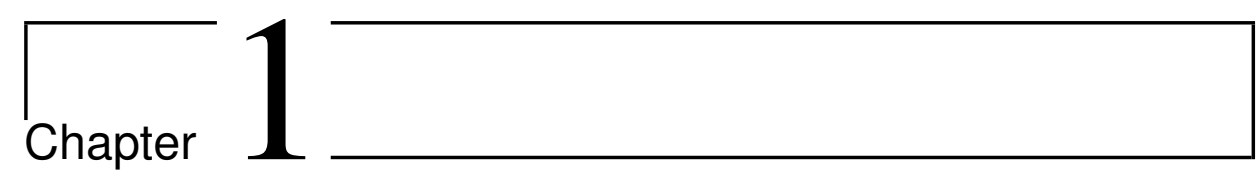

\section{Introduction}

The proliferation of IT in the past decades has been accompanied with an increase in information security risks. These risks affect a large number of people. Consumers can have their identity and credit card details stolen by criminals. Governments can lose secret data, either intentionally due to theft by insiders or by accident such as when USB sticks are lost. Enterprises experience constant attacks by hackers that impact the availability of the infrastructure on which they depend.

Why does the usage of IT go together with so many security risks? We provide a first answer to this question by explaining the concepts in a standard threat model [91]. First, there is the continued existence of vulnerabilities in IT systems. We do not really know how to build secure software, and when we can, it is not economically worth it to put effective safeguards. Second, even if the software is potentially secure, it is difficult and expensive to configure and manage it adequately, and to understand what the necessary protection requirements actually are. Third, there is the presence of threats created by adversaries, people who are motivated and creative enough to seek out and exploit these vulnerabilities. Fourth, the increased usage of IT makes it more tempting for adversaries to attack systems for their own benefit; there are more assets and their value has grown over time. Criminals simply go where people are, and if people are on the Internet the criminals will go with them. Thus, the risks of IT increase.

However this is only a partial answer. What we have witnessed in the past decades is not only the existence and exploitation of flaws in an ever larger set of IT systems. The nature of IT itself has also changed as systems are increasingly becoming interconnected and it is this change that is one of the major underlying causes of the increased vulnerability of IT systems.

In order to collaborate more with businesses partners, customers and clients, organizations expose larger parts of their processes to the outside world. Naturally 
this exposure leads to an increase in information security risks as more data and more vulnerabilities are exposed to outsiders. As the security barriers between organizations break down, the differences between insiders and outsiders blur. This phenomenon is called de-perimeterization. In this thesis, we will explore this phenomenon, examine possible treatments for the problems caused by it, and particularly zoom in on one of them: namely exploiting the security-relevant differences between physical and digital components. This chapter sets the stage for this research.

\subsection{Research Motivation}

The motivation for our research lies in the identification of two knowledge gaps:

1. De-perimeterization has not been defined clearly.

2. Most solutions for it are digital but ignore the physical aspects of it.

Regarding the first gap, although the concept of de-perimeterization is widely used it has not been thoroughly defined, and neither have the possible treatments of the problems caused by de-perimeterization. The term de-perimeterization was originally coined by Measham [60], and subsequently made popular by the Jericho Forum, an industrial consortium part of the Open Group. The main mission of the Jericho Forum is to help develop security standards that address the challenges of de-perimeterization. Since its conception in 2004, the Jericho Forum has published an extensive list of papers about the topic. This includes a list of commandments (security principles) [104], and a brief overview of their solution called the Collaboration-Oriented Architecture (COA) [107]. More recently they have also spoken out about cloud-computing [108] and explained their view on benefits and limitations of this computing model. Outside of the Jericho Forum, the concept of de-perimeterization has gained some attention from the scientific community as well 1 ]

Not surprisingly, our own investigation of de-perimeterization revealed a second research gap. The problem definition by the Jericho Forum is such that the emphasis is on technical and especially on digital solutions. However, outside of the digital domain, there are other solutions possible that are not explored. Especially, we take the view that de-perimeterization is enabled by the replacement of physical connections with digital connections. Physical connections are roads and doors that keep a physical distance or barrier intact. Digital connections break through these physical barriers, so that everything is connected, and

\footnotetext{
${ }^{1}$ A search on the Scopus bibliographic database in 2011 revealed 10 entries.
} 
traditional boundaries disappear. Thus, the security problems of de-perimeterization can be understood in terms of the differences between physical and digital connections. An effective treatment of de-perimeterization might very well lie in reapplying physical security techniques and making smarter choices (trade-offs) between physical and digital components, combining those properties of both domains that are most beneficial to security.

\subsection{Research Questions}

Having outlined the motivation for our research, we are now able to define the research questions. Our overall aim is to deliver results that help professionals and researchers improve information security in a de-perimeterized world by exploiting both physical and digital security mechanisms. This leads to the following research objective:

Understanding how system security can be improved by exploiting the differences between physical and digital security mechanisms.

To this end, we research first how IT systems cause security problems, or positively formulated, how and to what extent they can realize security goals. We then investigate physical system security. Finally, we examine physical and digital security together to understand their differences and how they can be combined optimally.

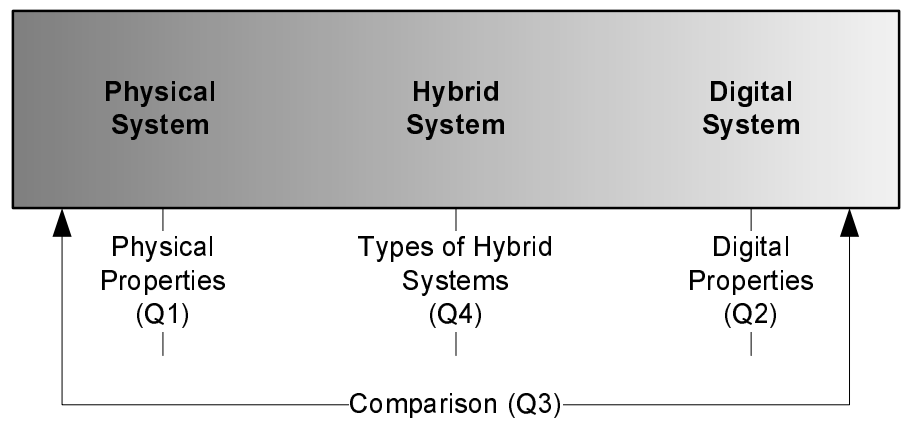

Trade-offs (Q5)

Figure 1.1: Simple conceptual framework about physical and digital systems in relation to the first five research questions.

Figure 1.1 shows a simple conceptual framework together with the first five research questions. In the figure all possible systems are being depicted on a hor- 
izontal bar, ranging from "completely physical" to "completely digital" 2 In the middle there is a category of mixed systems that we call "hybrid". As an illustration we consider voting in an election: a completely physical system is paper voting, with paper ballots and ballot boxes, whereas electronic (digital) voting replaces all of these by computers. In the middle of these two are hybrid systems such as computers printing paper ballots that voters deposit in ballot boxes.

From this framework, the research questions are derived:

RQ1: What are the properties of physical entities that physical security mechanisms depend upon?

RQ2: What are the properties of digital entities that digital security mechanisms depend upon?

RQ3: What are the key differences between physical and digital security mechanisms?

RQ4: How can physical and digital security mechanisms be combined?

RQ5: What are the trade-offs between physical and digital mechanisms?

RQ6: How can we apply the knowledge about physical and digital properties, combinations and trade-offs to improve security in an integrated way?

\subsection{Research Scope}

Having just defined the research questions, we will now consider the scope of our research and consider the limitations of our research. These limitations might not be apparent at first and to manage expectations of our results, we summarize them here.

A first issue is that as we compare physical and digital processes, we also compare situations before and after automation. Because automation is a historic trend, our research can be misunderstood as either a historical comparison or a study on the effects of automation. However it is not our intention to make a comparison between for example an office in the 1910s and one in the 2010s. To begin, much more has changed than automation between these two eras; The structure of organizations and the educational level of employees are very different and these differences would have to be included in the research to make a proper historical comparison. We are only interested in direct effects of automation on security. Our scope is thus limited to examining the differences of physical and digital systems concerning information security in the present.

\footnotetext{
${ }^{2}$ Naturally IT systems also have a physical basis, we will elaborate on this in Chapter 2
} 
Second, we have to consider the breadth of our research concerning these security-relevant differences. There are many different types of physical and digital systems in use. A priori, we deem it infeasible to research all types of systems, or to deliver results that apply to all of them. We cannot create an exhaustive list of physical and digital security mechanisms and their differences: compiling such a body of knowledge would require an enormous amount of time, and we do not see any option for proving that such a list is actually complete, nor do we know of any utility for such a list. Instead, we will examine a number of existing processes that are difficult to implement securely and that are realizable both physically and digitally. From these cases we try to extract mechanisms, which can be freed from a particular context and so generalized to a similar context, where this similarity should be operationalized.

Third, concerning the depth of our research, it is also impossible to consider the detailed workings of physical mechanisms down to the application of physical laws of nature. In fact, such research would also be difficult to utilize for information security practitioners, which is one of our objectives. The same holds true for digital systems that we cannot examine in detail down to the exact bits and bytes that are being processed or that constitute the computer program itself. We must base our investigation about the physical and digital domain on relatively simple mechanisms and properties. (See also Chapter 2 where we further explain the relation between high-level policies and low-level mechanisms.)

Fourth, as a person is also a physical entity, her behavior, both as an individual as a member of a group can potentially be placed under physical security. Social processes can also be relevant for digital security mechanisms, for example concerning monitoring activities that would trigger a response from an analyst working in a security operations center. When relevant, we will discuss how persons collaborate together on security relevant tasks, but we define psychological processes or the dynamics of social systems to be out of scope.

\subsection{Research Design and Methods}

With the scope defined, we can provide an overview of the research design and methods, which are explained in more detail in Chapter 5. To begin, the design consists of nested case studies. At the top level we investigate four particular types of systems, namely access control, voting, IT infrastructure and rights management. These systems have either known security problems (voting, IT infrastructure or rights management) or are used to secure other systems (access control). Within each such case we have three sub-cases:

- physical systems

- digital systems 
- hybrid systems (systems that combine physical and digital components)

We will now explain the data collection, representation and analysis methods bottom up. First, within each sub-case, data collection is done using literature studies, the study of primary documents, and interviews on-site. Regarding literature studies, we base our approach on the method described by Webster and Watson [223]. Here, literature is retrieved from well-known sources such as leading journals and additional literature is found by tracing back the cited papers and forward towards conference papers. The findings are presented concept-centric, meaning that all literature on a certain concept is discussed in one section.

Second, we performed an inquiry into selecting a way to represent systems in a uniform way across all case studies. In the end we chose KAOS because it has four important elements:

E1 Relate low-level system structures to business goals.

We related specific entities and process steps to business goals.

E2 Reason about social and physical system context.

This concerns the domain properties (digital and physical) that are identified in the case studies.

E3 Express violations of security goals.

This concerns the actual attacks that can take place.

E4 Express multi-step attacks.

This concerns series of steps that an attacker executes to attack a system.

Third, within each top-level case, data from the physical, digital and hybrid systems is compared in a cross-case study analysis. The results of this analysis answer the research questions on the differences of physical and digital system (RQ3) and their trade-offs (RQ5).

Fourth, throughout the case studies, data is analyzed in a step-wise fashion, where an initial hypothesis is checked against all available data. If the hypothesis does not fit the new data it is changed, particularly by altering the conditions under which it holds true. This is important because the effects of security mechanisms in a system can depend on specific conditions, either contextual or as part of the system. This approach is similar to analytical induction [180] and is used for the case studies. Thus at the end of case study four all knowledge developed from the case studies is codified.

After completion of the case studies, the results are used to construct two methods that can be used directly by practitioners (RQ6):

1. Method for analyzing the security of existing systems.

We present a method for using the identified physical and digital properties to identify threats and vulnerabilities. 
2. Method for designing new systems.

We present means to integrate physical and digital security mechanisms (the hybrid systems) as security patterns [51], based on the taxonomy developed in answer to (RQ4).

These results are then validated in a focus group meeting [138]. Figure 1.2] illustrates the research design and validation.

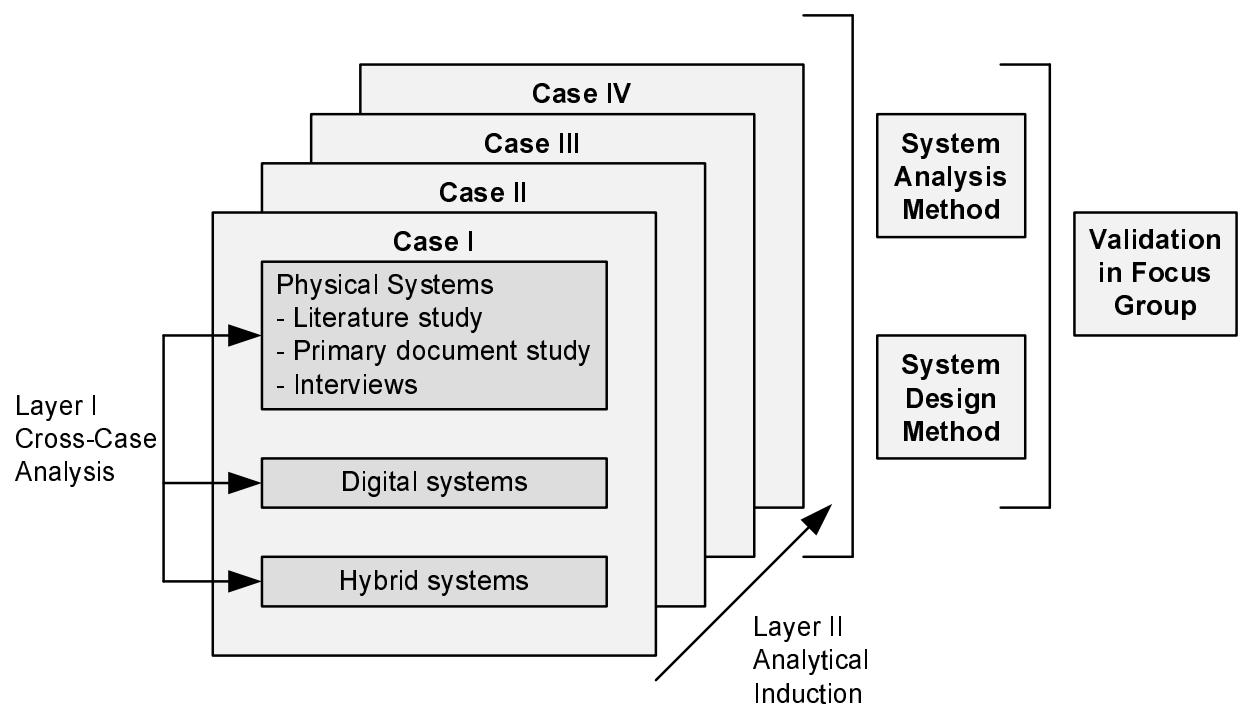

Figure 1.2: Research design with layered case studies.

\subsection{Contribution to Knowledge}

Our research contributes to knowledge in several ways:

- We provide a deeper understanding of the concept of de-perimeterization, what it is and what possible solutions are.

- We present mechanisms for analyzing, explaining and predicting the workings of physical and digital security.

- We present a set of properties of physical and digital entities that physical security depends upon.

- We present a taxonomy of hybrid systems, which combine physical and digital components.

- We present insight into the physical-digital security trade-offs for the goals of confidentiality, integrity and availability. 


\subsection{Implications for Practice}

The knowledge that results from this is useful for information security practitioners in three ways:

- We provide conceptual solutions for designing systems to minimize the security impact of de-perimeterization.

- We provide a method for analyzing the security of systems with digital and physical components based on security properties.

- We provide a method for designing secure systems with digital and physical components based on patterns.

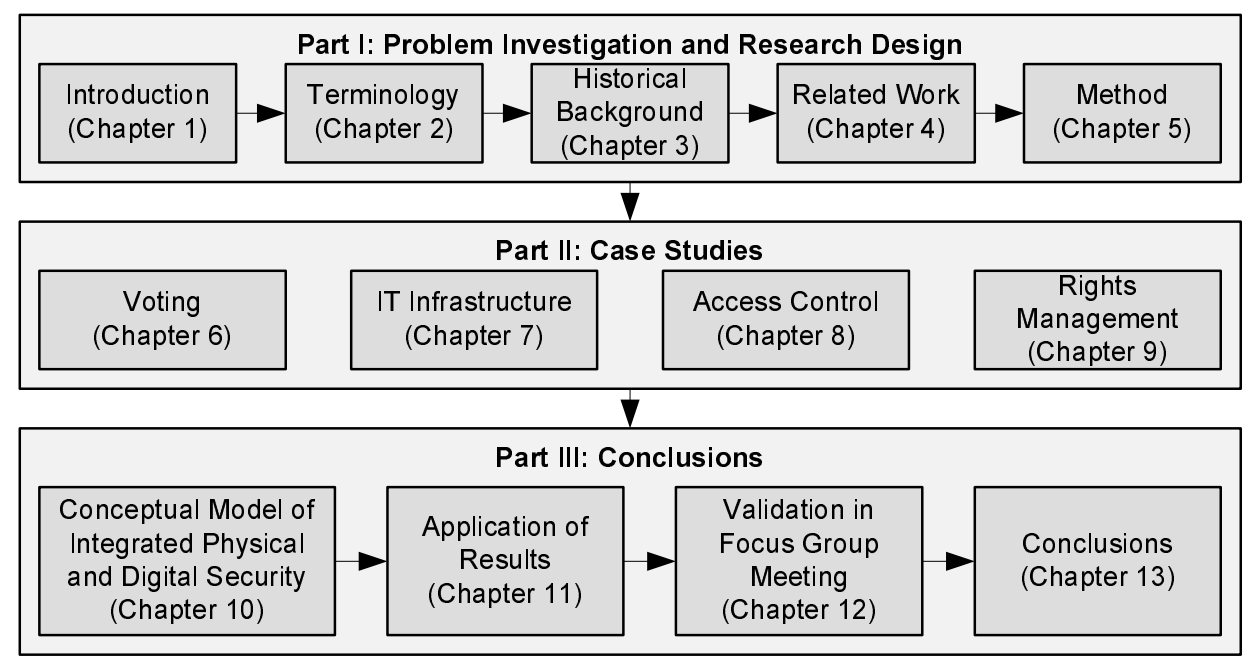

Figure 1.3: Outline of the thesis.

\subsection{Outline of the Dissertation}

Finally, we show the outline of the thesis in Figure 1.3. The thesis consists of three parts. In Part I, we introduce the context of our research. Part II contains the detailed methods and research findings, and Part III holds the system analysis and design methods, validation and conclusions.

\subsection{Summary}

In this chapter, we gave an introduction to our research, which concerns the security problems caused by de-perimeterization. We briefly explained the de-pe- 
rimeterization concept, outlined our research motivation and listed the research questions. Next we discussed our methodology and explained our terminology. Furthermore we explained the usefulness of our findings for exploiting the securityrelevant differences between physical and digital components. In the next chapter, we will further investigate terminology regarding information security as well as the physical and digital world. 


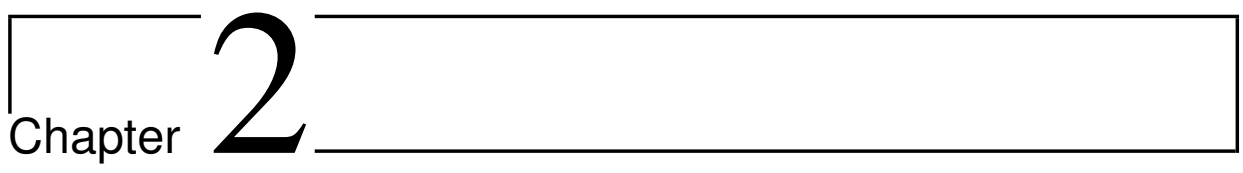

\section{Terminology}

\subsection{The Physical and the Digital World}

Previously, in our comparison of physical and digital processes we implicitly assumed that the physical and the digital world are clearly separated. That is not strictly true as everything in the digital world is ultimately physical. Computing is physical because without a physical processing unit, without electric current, there can be no digital world. All digital systems are thus physical-digital systems. However, there is a need to reason about the digital world independently from the physical world. The digital world concerns the storage, processing and communication of information represented in bits and bytes and the understanding of this does not usually require knowledge of the physical world, which concerns physical matter. (Exceptions where the digital world does require physical knowledge are related to for example energy usage, user interfaces and printers.)

Properties of the digital world are imposed on it rather than derived from the physical world. The physical and the digital world are domains: parts of the world that are convenient to treat as a whole [226]. Underlying the convenience to speak about the digital domain are several assumptions about the physical implementation. First, the physical implementation should not break down. Second, the physical implementation should be powerful enough for the system that executes on top of it (for example in terms of processing speed or storage capacity). A complication arises when the digital system does not simply use the physical world but is designed to interact with it. For example in an industrial processing system, a digital system controls a physical pump and it monitors the physical flow through a pipe. In such cases the digital and the physical domain are difficult to treat conveniently as separate worlds, because the understanding of the entire system requires knowledge of both domains. 
This also depends on how one defines the boundaries of a digital system; the larger the system is defined to be, the more likely it is to have interactions with the physical domain. In the example of the industrial processing system, a narrow system definition would include only the software communicating with the pump process, whereas a wider definition could include the pump itself as well as the person operating it.

Figure 2.1 illustrates the relations between the physical and digital domain.

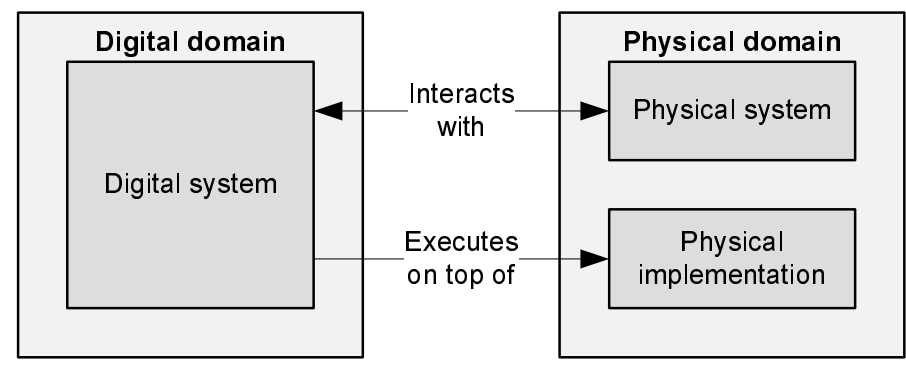

Figure 2.1: The relations between the physical and digital domain.

Concerning the research question about the trade-offs between physical and digital systems (RQ5), what does it mean to choose between a physical and a digital implementation? This is a choice between a system that can be understood purely in terms of its physical behavior and a system that can partly be understood by its digital behavior, which is made possible by the underlying physical system. For example we have the choice between protecting a secret by storing it in print inside physical vault (a physical security mechanism), or by storing it as an encrypted file on a computer system (a digital security mechanism).

\subsection{Digital Systems}

We will now consider terminology related to the digital domain. This is necessary, as our research concerns several areas in which different terms are used. A first set of terms is closely related to "digital" and consists of the adjectives logical, electronic and cyber. Originally these terms have different meanings:

- "Digital" emphasizes the discrete properties of a system, in contrast to continuous or analogue properties. (digital sound processing, digital rights management)

- "Electronic" (or "electric" or "e") emphasizes the fact that electrons play a role in a system, such as in electric current. (e-mail, electronic voting)

- "Logical" emphasizes the formality or the manipulation rules of a system. (logical security mechanisms, logical access control) 
- "Cyber" emphasizes the control of a system. A cyber-physical system is a controlled physical system.

Although related, these terms do not necessarily refer to the same. For example, a digital system does not have to be electronic because we can transfer data through optical fiber rather than through a copper wire. Likewise, there are electronics that are not digital, such as for receiving FM radio signals. A cyber-physical system can be controlled through analogue rather than digital electronics. In colloquial speech, the distinctions blur: e-mail is e-mail regardless of the medium through which it is transferred or on which it is stored. We will consider all of these terms as equivalents of "digital". In the remainder of this thesis, we simply use the most appropriate terms for each case, such as "electronic voting", or "digital rights management". If however there is a choice we will use the term "digital".

A second set of terms (related to research question RQ4 about trade-offs) concerns three types of system transformations from physical to digital.

- "Automation" involves replacing a physical system with a digital system.

- "Virtualization" concerns the construction of a system using software from underlying physical resources. For example a virtual private network (VPN) is a private network created from the shared resource Internet.

- "Simulation" concerns the imitation of an existing process using software.

Again being aware of these differences and similarities, we will use the most appropriate term for each case study in our research.

\subsection{Information Security}

It is necessary to investigate information security as it is the subject of our research. We examine two things:

- the embedding of security goals in an organizational context

- the operationalization of security goals themselves

\subsubsection{Embedding of Security Goals in an Organizational Context}

Ensuring security should be a top down process, where business requirements determine how assets should be secured. The motivation for this is that budgets are always finite, and the importance of an information asset should determine how much effort is put in protecting it. If the information asset is critical to the organization, it should be better secured than if it is unimportant. This top-down process begins with establishing broad, organization-wide information security policies 
(for example based on ISO 27001) that link information security to business requirements - the "why" of information security. Next, for each system or type of data, more detailed policies are established, which specify how they should be handled. In turn, these are translated into lower level policies (passwords should expire after 6 months), down to procedures (make a list of non-expiring passwords every 6 months) and technical implementations (the actual configuration of an identity and access management system). These implementations are the "how" part of information security. In Chapter 5, we will explain the focus our the research regarding the "why" and the "how".

\subsubsection{Operationalization of Security Goals}

Whereas terminology about physical and digital systems is ambiguous, the operationalization of information security goals is more straightforward because ISO standards offer precise definitions for information security. To begin, ISO defines information security as the preservation of confidentiality, integrity and availability of information [92] 1] Other concerns include authenticity, accountability, non-repudiation and reliability.

Confidentiality, integrity and availability (abbreviated the CIA properties) are defined in ISO standard 7498-2:1989 [90]:

- Confidentiality: the property that information is not made available or disclosed to unauthorized individuals, entities, or processes.

- Integrity: the property that information has not been altered or destroyed in an unauthorized manner.

- Availability: the property of information being accessible and usable upon demand by an authorized entity.

Information security has other aspects that are not directly related to data [91]:

- Authenticity: the property that ensures that the identity of a subject or resource is the one claimed.

- Accountability: the property that ensures that the actions of an entity may be traced uniquely to the entity.

- Non-repudiation: the ability to prove that an action or event has taken place, so that this event or action cannot be repudiated later. [90].

- Reliability: the property of consistent intended behavior and results [94].

${ }^{1}$ Cf. Avizienis et al. [9] for a taxonomy of security properties. 
A meta-goal that can be added is assurance, the confidence of actors that these properties actually hold.

Authenticity, accountability and non-repudiation are of especially great importance in a de-perimeterized world, where it is difficult to assess identities of systems and individuals, hold them accountable and prevent them from denying their involvement in transactions.

As for privacy, this is a goal related to confidentiality. We define privacy as "ownership and control of personal information" [76]. In this view, we see privacy as one specific type of confidentiality, when it concerns a specific person that is interested in owning and controlling access to information about her. As such, the concept of privacy has no meaning for a corporation, we can only speak of company confidentiality, and not of company privacy.

\subsubsection{Differences between Security Properties}

Turning back to security properties, it is important to realize that these properties are very different, especially when considering trade-offs between physical and digital security mechanisms: we cannot perform trade-off analysis with the objective to optimize the general effect on information security, without detailing which properties are concerned. Without discussing technicalities, we will consider three such differences for the CIA properties, related to (i) realizing these security goals, (ii) detecting violations of these goals and (iii) recovering from violations.

Goal Realization Concerning the realization of these goals, ensuring confidentiality of data implies limiting the number of copies in circulation, as even one stolen copy is sufficient to cause a confidentiality breach. Alternative approaches are to spread partial copies between different systems to prevent a single malicious entity from retrieving the whole, or to encrypt the data and distribute the storage of data and the key management over different systems to prevent collusion. Integrity is realized by many different techniques in use for transaction systems, such as double record keeping and checkpointing (keeping parts of a transaction $\log$ and validating series of transactions to prevent a system to enter an invalid state). In contrast to confidentiality, availability is realized by keeping as many copies as feasible, and separating them as much as possible.

Detecting Violations As for the detection of violations of these goals, the confidentiality of information is hard to prove by itself. Simply by having access to the raw information we cannot know, by looking at this information, who else might have had prior access to it. Rather, we require contextual information such as logs about the live system handing the information. Alternatively, we can use a security proof that the software handling the information satisfies our confidentiality 
goals (Cf. Jacobs et al. [100]). Reading data does not alter its state, therefore we never know if someone accessed it (unless other mechanisms are put in place). It can be disproven easily; When someone knows something she is not entitled to, it is clear there is a breach of confidentiality. The difficulty of proving and disproving the integrity of data is entirely context dependent: a draft email can be easily changed without this being detected, whereas manipulation of a photo or a bank account is detectable in many cases. Availability is the easiest to prove and disprove, as we can simply try to access the information.

Recovery Recovery is also different for the three properties. Unless in specific situations, recovering from a confidentiality breach is impossible as data can be published on the entire Internet and cannot be removed thereafter. Recovery of a breach of integrity is only possible when there is a prior version or a transaction $\log$ that is known to be correct. Loss of availability of data at a certain moment can be undone through restoring a backup.

\subsubsection{Dependencies}

Although distinct, the CIA properties are dependent in real systems. First there is a logical dependency: before integrity and confidentiality of information are of concern it must be available in the first place. Second, attacks can involve violation of different CIA properties for different types of information. For example an administrative password must remain confidential, because with it the access records can be changed (violating the integrity of the access control system), so that someone can access specific data that she would not be allowed to normally (confidentiality). After the attack, the hacker removes any traces from the system log (violating integrity). Whenever possible, we will constrain ourselves to a first order analysis of security impacts and exclude long chains of attacks from our results. With this in mind, we continue to the next chapter about the historical background of de-perimeterization. 


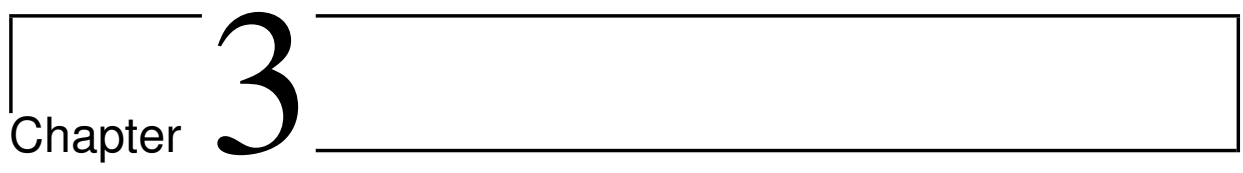

\section{Historical Background}

\subsection{Introduction}

In 2010 two landmark events showed how incredibly difficult it is to protect information systems 1 Below, we will give an overview of what occurred, analyze these events in further detail and place them in the context of de-perimeterization 2

The first event was the publication of over 250,000 diplomatic messages from US Embassies by the Wikileaks organization [202]. Reportedly, an US army soldier named Bradley Manning stole these documents from SIPRNet, a closed network maintained by the US Department of Defense.

The second landmark event was the Stuxnet computer attack on Iranian nuclear installations [201], specifically on the Bushehr nuclear plant. Reportedly, a sophisticated software program, attacked the software controlling the centrifuges needed by Iran to enrich uranium. This resulted in the destruction of about a fifth of these devices.

These events show the extraordinary weakness of information systems and networks. First, even the United States, one of the most technologically advanced nations, was found to be incapable of keeping its secrets. Second, Iran, being well aware of the likelihood of attacks on its nuclear facilities, could not protect its computers. These are extreme cases that we can use to generalize: both the United States and Iran had a tremendous interest in protecting their systems, and especially the United States should have had enough resources to accomplish this. Following this line of reasoning, because they both failed, it is unlikely that any other nation (or enterprise) can succeed in keeping information secure when faced

\footnotetext{
${ }^{1}$ This chapter is based on Van Cleeff and Wieringa 211

${ }^{2}$ Given the enormous interests of parties involved, we cannot know for certain that these events exactly took place in the way we describe. The accounts given here are based on public sources that we deem credible.
} 
with a determined and resourceful adversary. Is it then impossible to protect secrets in the digital age? There may be specific factors involved in both cases, which are not present in other situations. In the next sections, we will further analyze these events in an attempt to uncover the underlying mechanisms that caused them and find potential solutions for these and other security incidents.

\subsection{De-perimeterization}

Our analysis starts with the observation that in both cases the boundaries between organizations, and between organizations and persons had broken down. As for Wikileaks, this event was precluded by the analysis that after the 9/11 attacks, the US intelligence agencies were not sharing enough data with each other to prevent terrorist attacks. To "mitigate" this lack of information sharing, an architecture was designed to facilitate information exchange between several organizations, including the US Department of Defense and the US State Department. The risk that this sharing would cause confidentiality breaches was well known, and was simply the "cost of doing business" [14]. Crumbling security perimeters (this time being unable to keep attackers outside) were also present in the case of Stuxnet. A likely source of the infection were Russian consultants from contractor Atomstroyexport, who were involved in the construction of Iran's nuclear powerplant [229]. Even without being aware of it, the consultants could easily have infected the plant's computer systems because the virus spread via USB sticks.

Thus, these security breaches can be placed under the umbrella of what Bruce Schneier called the ugliest word in IT [190]: de-perimeterization, or the dissolution of the boundaries between an organization's internal and external network. This term was made popular by the Jericho Forum - an industry consortium part of the Open Group, who defined it as "the erosion of the network perimeter" $[106]^{3}$ In 2004, the Jericho Forum observed that security approaches made two assumptions about their environment that were increasingly becoming invalid [103]:

- Organizations own, control and are accountable for the IT systems they use.

- Every individual is employed by exactly one organization.

To better understand the problem, we examine the history of de-perimeterization and Figure 3.1 shows the Jericho Forum's view of its historical development [105]. According to this model, the trend is toward more connectivity. Technologies and business processes changed and increased collaboration led to more sharing of information. In a networked world "inside" and "outside" could

\footnotetext{
${ }^{3}$ In other cases, members of the Jericho Forum have also defined de-perimeterization as a potential solution. For an overview of terminology and definitions we refer to Walker [221] and the Forum itself [59], [103], [196].
} 
no longer be clearly distinguished. Connectivity between systems increased, became more flexible and harder to track and understand.

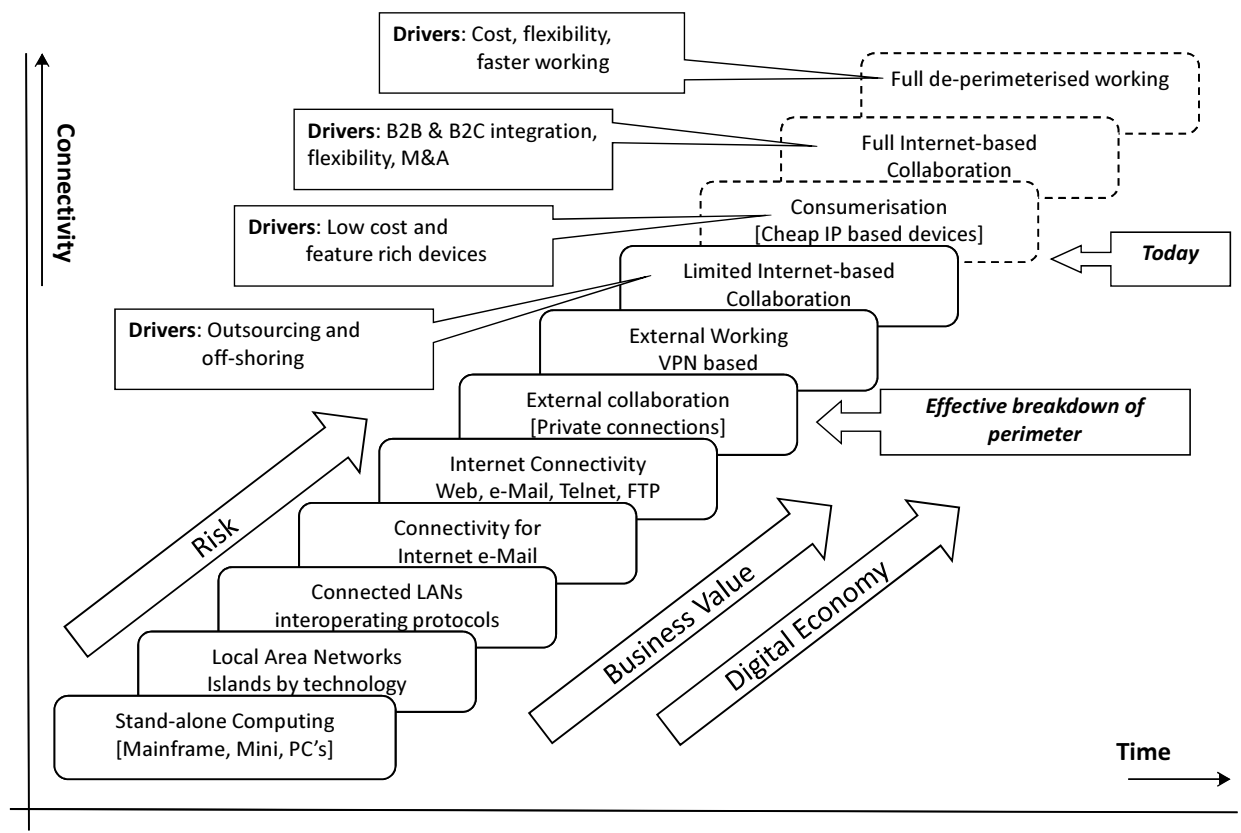

Figure 3.1: The Jericho Forum's view on de-perimeterization [103].

Negating the aforementioned two assumptions, we can state that de-perimeterization occurs when (i) an organization does not own or control its IT infrastructure or is not accountable for it, or (ii) when individuals are either self-employed or hired by more than one organization. We distill two essential elements of deperimeterization as explained by the Jericho Forum:

- There is increased system connectivity between organizations. The reason is that de-perimeterization involves multiple legal entities that jointly own, control or are accountable for information systems.

- The security effects of the increased system connectivity (and possibly even the connection themselves) are unknown. This is caused by the shared responsibilities between organizations for systems.

We will illustrate this by several examples. First, a classic example of de-perimeterization occurs when organizations outsource part of their IT infrastructure. In such a case, there are people in the outsourcing provider that have access to the servers and the data of the outsourcing client. These people are thus neither completely inside or outside the outsourcing client, and we can call them "external insiders" [61] of the client, or persons with specialized access. In an outsourcing 
situation, both the outsourcing client and outsourcing provider have partial access to each other's systems. Of course, each of their systems is in turn connected to other systems and neither the outsourcing client nor the outsourcing provider has a complete overview of these connections. Second, a recent phenomenon is cloud computing [83], where an IT service provider shares its IT resources among customers. As such cloud computing is different from traditional outsourcing situations where each customer has dedicated and tailored systems. As in outsourcing, the cloud provider has full access to the data stored by these customers. Additionally, in case of security flaws, customers can also have access to each other's data. For example, customers from Amazon's EC2 cloud computing platform were able to map Amazon's internal network and detect the presence of other customers [176].

\subsection{Analysis of De-perimeterization}

De-perimeterization threatens organizations in several ways. First, the unknown dependencies can be the source of new security problems. In a networked world, it is not hard to create very complex systems of systems, which work flawlessly for some time. However, these systems have structural dependencies that only become apparent when something goes wrong. For example, in 2007, the Skype communication service went offline for some time after its users massively rebooted their Microsoft Windows machines on which they had installed Skype, following the release of a Windows update [186]. In this case, the perimeters of Skype users, Skype itself and Microsoft clearly blurred. Obviously, Skype was dependent on the patching process of Windows, but this was unknown before the event. As de-perimeterization continues, such events become more likely.

Second, assessing security risks becomes harder. At the technical level, we want to assess the security of IT products, and this is possible using the ISO/IEC 15408 framework, also known as the Common Criteria (CC) [38]. The CC assumes that there is an identifiable product or system to be evaluated, called the Target Of Evaluation (TOE). Once evaluated, the product can receive a certification that it has certain desirable security properties. Potential customers can then check whether their own requirements can be satisfied with the product. Unfortunately, this approach is undermined by the fact that the benefits of a certified product are less clear in a networks of systems: a composition of certain security products is not necessarily secure itself, and networks are likely unique and dynamic. For example, an IT service provider might want to change its employee authentication mechanisms, but fail to understand the impact of this for its customers, to which its employees have access. Additionally, the customers will also change their systems over time about which the service provider has little 
knowledge. Thus, unknowingly, the authentication service's effectiveness might be reduced.

A similar problem is faced when certifying organizations. For example the ISO 2700x series [92]-[94] specify a long list of security requirements that an organization should meet in order to be "in control" of its information security. This requires a wide range of technical and organizational controls, such as authorization procedures and hiring practices. One of these concerns the assessment of connections with third parties (Section 6.2 in ISO 27002:2005), but the standard does not specify in great detail what the requirements should be for these organizations. In a de-perimeterized world, it is therefore questionable that an organization that has an ISO 27001 certificate is really in control, as it may depend on many other organizations for this, and it might not even know these dependencies 4

In order to effectively secure today's information systems and build the next generation securely, we need to understand better how de-perimeterization occurs. Our view differs from the Jericho Forum's view. First, we believe that de-perimeterization is not a linear trend towards more connectivity but rather a cyclic process of connecting and disconnecting systems. Second, de-perimeterization cannot be understood as a pure digital phenomenon, as it essentially entails the exchange of physical connections for digital connections. Digital networks break down boundaries between systems that were once isolated. We next substantiate these claims.

If we examine the history of the Internet, we can indeed find that once physically isolated systems of universities and businesses were connected. But this brought along security problems, and the networks were subsequently separated using firewalls. This process took over two decades, as the Internet started in the 1960s and the first commercial firewalls (that separated organizations from each other) appeared in 1989 [179]. Over time, the internal organizational networks themselves grew until they were too big to be secure: they had to be compartmentalized again. Finally, new technologies such as mobile devices and the service-oriented-architecture led to new connections, and we can expect new security perimeters to be established for these in the future.

In contrast with the view of the Jericho Forum, we believe thus that de-perimeterization is not a straightforward cumulative process towards more connected systems, which ultimately become completely de-perimeterized. Instead, as connectivity increases, we observe a cyclic process in which the basic trade-off between accountability and business opportunities are balanced based on risk assessments. Our version of events is depicted in Figure 3.2. The drivers towards

${ }^{4}$ ISO is currently working on a standard about information security for supplier relationships, ISO/IEC 27036-3 [95]. 
de-perimeterization replace physical by digital connections, whereas the drivers limiting de-perimeterization replace digital by physical connections.

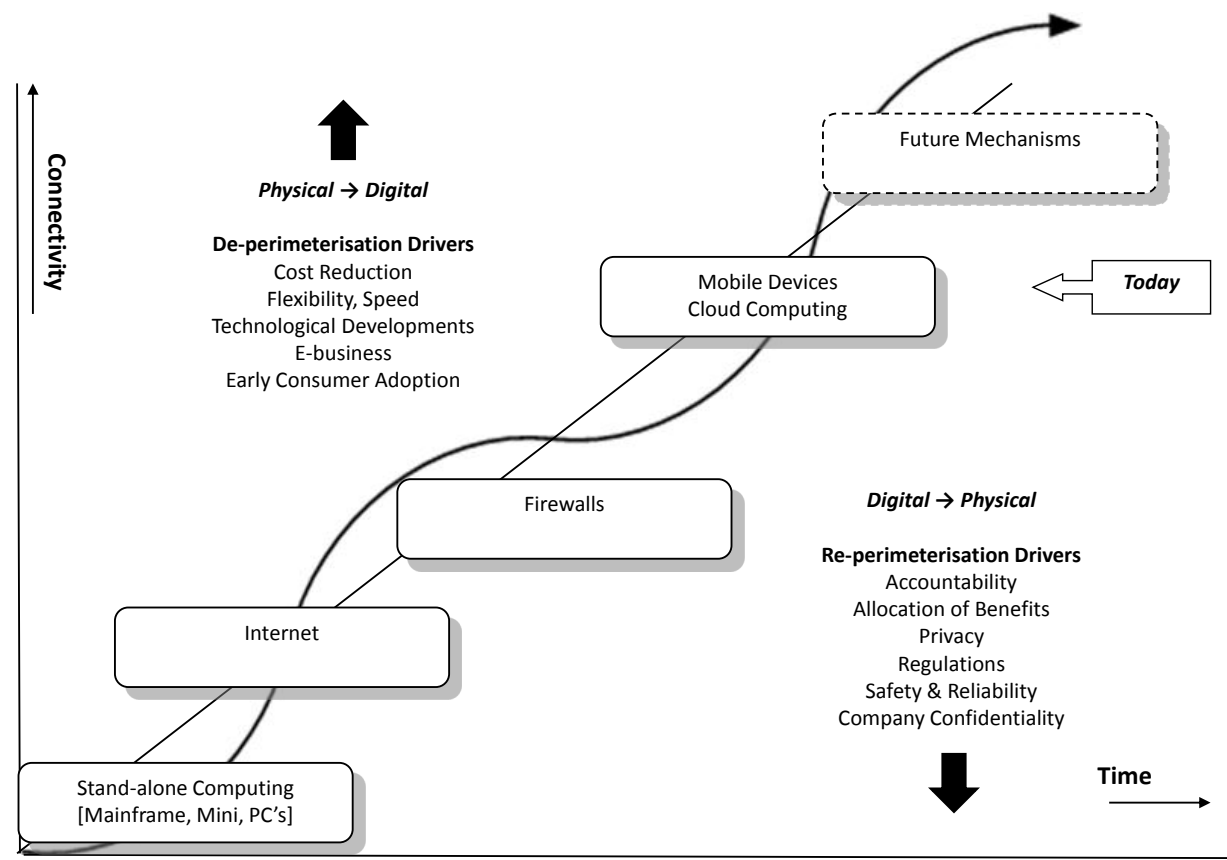

Figure 3.2: Alternative view on de-perimeterization.

The oscillation is due to the interplay of two sets of opposing forces, one in the direction of connectivity (de-perimeterization), and one in the direction of reperimeterization. The forces in the direction of connectivity include the desire for cost reduction, flexibility and speed of business, facilitated by technological developments. Enterprises see the advantages of e-business and open up their networks. Universities want to develop and utilize the new infrastructures for their own research. For many new technologies, their impact on security is not clear. Organizations and individuals that are not aware of this, or do not consider it a problem (such as consumers), will take the risk and adopt the technologies. IT is first made to work and there is no incentive to invest early on in security features. (Recent technologies that drive de-perimeterization include smartphones, tablets as well as virtualization and cloud computing.)

Once technologies become mainstream, different forces come into play, leading to re-perimeterization. Vulnerabilities will be found, risks will increase and potential users will demand security features to be fitted to the new technology, before integrating it in their systems. Additional forces for re-perimeterization thus include the need for accountability, proper allocation of benefits in a business network, privacy, safety and reliability and company confidentiality. Then the in- 
dustry creates a new wave of products and services and the cycle starts again. Our prediction is that this oscillatory trend, rather than the linear Jericho trend, will continue because none of these forces in play will go away.

Even though we cannot "solve" de-perimeterization, we might still be able to manage it. In the next section, we will examine several approaches for dealing with de-perimeterization, and select one of these to investigate in more detail in the remaining chapters.

\subsection{Treatment of De-perimeterization}

To manage de-perimeterization, the Jericho Forum initially published a set of eleven "commandments" [104] or principles, shown in Figure 3.3.

\begin{tabular}{|c|c|c|}
\hline Category & \# & Description \\
\hline \multirow[t]{3}{*}{ Fundamentals } & 1 & $\begin{array}{l}\text { The scope and level of protection should be specific and } \\
\text { appropriate to the asset at risk. }\end{array}$ \\
\hline & 2 & $\begin{array}{l}\text { Security mechanisms must be pervasive, simple, scalable, } \\
\text { and easy to manage. }\end{array}$ \\
\hline & 3 & Assume context at your peril. \\
\hline \multirow[t]{2}{*}{$\begin{array}{l}\text { Surviving in a } \\
\text { Hostile World }\end{array}$} & 4 & $\begin{array}{l}\text { Devices and applications must communicate using open, } \\
\text { secure protocols. }\end{array}$ \\
\hline & 5 & $\begin{array}{l}\text { All devices must be capable of maintaining their security } \\
\text { policy on an un-trusted network. }\end{array}$ \\
\hline \multirow[t]{2}{*}{ The Need for Trust } & 6 & $\begin{array}{l}\text { All people, processes, and technology must have declared } \\
\text { and transparent levels of trust for any transaction to take } \\
\text { place. }\end{array}$ \\
\hline & 7 & Mutual trust assurance levels must be determinable. \\
\hline $\begin{array}{l}\text { Identity, } \\
\text { Management, and } \\
\text { Federation }\end{array}$ & 8 & $\begin{array}{l}\text { Authentication, authorization, and accountability must } \\
\text { interoperate/exchange outside of your locus/area of con- } \\
\text { trol. }\end{array}$ \\
\hline \multirow[t]{3}{*}{ Access to Data } & 9 & $\begin{array}{l}\text { Access to data should be controlled by security attributes } \\
\text { of the data itself. }\end{array}$ \\
\hline & 10 & $\begin{array}{l}\text { Data privacy (and security of any asset of sufficiently } \\
\text { high value) requires a segregation of duties/privileges. }\end{array}$ \\
\hline & 11 & $\begin{array}{l}\text { By default, data must be appropriately secured when } \\
\text { stored, in transit, and in use. }\end{array}$ \\
\hline
\end{tabular}

Figure 3.3: Jericho Forum Commandments [104].

Next, they provided a solution framework called the "Collaboration-Oriented Architecture" (COA) [107]. The COA framework consists again of set of principles, as well as key processes to be managed, services that must be offered, attributes to be measured (KPIs) and finally supporting technologies. For the de- 
tails we refer to the white papers of the Jericho Forum. As a general direction, the Jericho Forum emphasizes protection of the smaller and more valuable units of IT systems. In the information age, this means a focus on data security rather than network security. The final phase of de-perimeterization is thus accompanied by data level authentication, where "data is inherently secured and will only operate in validated secure environments by authorized people" [196].

There is no proof whether the COA will actually help to manage de-perimeterization, in the first place because parts of it are still in the conceptual phase, and second because it too broad to be validated as a whole. Concerning technical (IT) solutions, we will therefore focus on the possibilities for perimeter placement and especially the options for data-centric security (Section 3.4.1), as this solution would be used in the last phase of de-perimeterization.

In addition to providing definitions of what de-perimeterization entails, we will also examine potential treatments. This leads to a total of three conceptual solutions in the digital, legal and physical domain. Concerning the digital domain, apart from perimeters around an organization's own IT infrastructure, there are many other places to put perimeters, for example at an Internet Service Provider (ISP) or around individual devices. As for the legal domain, de-perimeterization involves different legal entities. By changing organizational boundaries of responsibility, accountability, and right to know we can thus also influence de-perimeterization. For example if two organizations require access to each other's systems (and are thus de-perimeterized) we can create a new legal entity that encompasses these systems and is not de-perimeterized, or to a lesser extent. Finally, we can view de-perimeterization as a trend enabled by automation, which is the replacement of physical processes and entities by digital processes and entities. To limit de-perimeterization we can therefore also de-automate some of these processes and entities. Next we will discuss these and other treatments in further detail, remaining at the conceptual level.

\subsubsection{Digital Domain}

\section{Data-centric Security}

The Jericho forum has proposed to go with the flow of de-perimeterization by placing a perimeter around the data, rather than around the entire infrastructure of the organization ( [104], [158]) ! $^{5}$ Agrawal et al. [1] and Grandison et al. [75] propose several technical mechanisms to realize data-centric security. There are various arguments for implementing security on the data itself. Firstly, as stated by Grandison et al., security must be data-centric because only the data has real business value: the IT infrastructure of an organization is of limited value if there is no sensitive data on it (meaning data of which the loss of confidentiality, in-

\footnotetext{
${ }^{5}$ The Jericho Forum has now renamed data-centric security to "micro perimeterization" |109|.
} 
tegrity or availability would cause serious problems). Secondly, it does not make sense to have a security perimeter include more than is strictly necessary. In general, perimeters do not scale. If a system grows, the difference in security levels between the inside and the outside diminishes. On a network of ten servers all of them could possibly be trusted, but this is not the case if the network is expanded to include a thousand servers. Thirdly, business opportunities must be considered. The Jericho Forum argued that organization-level security perimeters actually hinder business rather than facilitate it. To enable cooperation with other organizations it makes sense to facilitate access to IT assets at the lowest possible level, and use these assets as building blocks for new business constellations. Fourthly, there are many situations in which organizations change shape, whereas the data will remain relatively stable. For example, a hospital might be reorganized and restructured many times, whereas there will be little need to change existing data about patients. Placing security policies at the data level can be a form of futureproofing security. Wherever the data will be in the future - it will be known how it should be secured. We identified two main types data-level security.

Database-centric The illustrative example here is the Hippocratic database [1], which takes its name from the Hippocratic oath that doctors must take to protect their patients and keep their information confidential. Aided by strong security policies and mechanisms, a database should adhere to the same principles. On top of it, other applications can be built. Because they derive their data from the Hippocratic database, they automatically adhere to the database's policies. The principle is used in client-server solutions where valuable data is stored at the server that enforces the security policies.

Free-flow In contrast to the Hippocratic database, where the data is stored centrally, we can associate a data item with "sticky policies" [10] that cannot be separated from the data, no matter where it goes. Sticky policies require a trusted environment in which the data can flow; provided by special hardware (trusted platform modules or TPM), or more general by encrypting data. Attribute Based Encryption (ABE) schemes allow data to be encrypted in such a way that specific policies are automatically enforced (Cf. Cheung and Newport [31]).

Limitations of Data-centric Security We identify four main issues with datalevel security:

1. Accountability checks at the organizational level remain necessary. Even though information flows freely between organizations, organizational accountability still exists: an individual organization, as a distinct legal entity, has to pay tax over its assets and receives accreditations and certifications. These activities require knowledge about what is inside and what is 
outside of the organization and who owns which data. For example, a bank is accredited as a whole for performing financial transactions and it will be required to prove its performance at the organization level. Some form of organizational perimeter should always exist.

2. The security of data itself cannot be assessed at face value.

Data is always generated by applications and by users, whose interactions can be very complex. If we can only study the end result, we cannot assess its confidentiality or integrity. We need to understand the computational and business processes that created the data. As a practical example, consider the security mechanisms for a banking application: first, observe that data integrity cannot be determined at the level of individual bank accounts: if a sum of money is transferred, integrity is at least determined at the level of those accounts involved: the total amount of money before and after the transactions should be the same. In fact the expected level will be higher because we also must consider the interest that is generated by the two accounts and the time it takes to transfer the money between the two accounts. Thus, accounting principles such as double-entry bookkeeping should be incorporated in data-centric security, leading to coarser grained security mechanisms.

3. Data needs to be exchanged with other systems.

Even if we can store data securely in a Hippocratic database or in a trusted environment, we will still need to export it at some time: in a networked world, communication of data with other organizations and individuals is a necessity and not everything can be placed under control of one agency. Again considering the example of banks, we must be able to transfer money between different banks. However, assessing the security measures of the other parties is beyond the capabilities of any database. Thus the problem of maintaining a secure data exchange cannot be addressed at the data level.

4. Encryption is not a panacea.

Encryption of data in the centralized or free-flow model cannot fully protect data. A first problem is that encryption requires key management, and thus shifts the problem to managing key data [21] (something the Jericho Forum also observed). For individuals key management is relatively easy. For example, they can encrypt their entire laptop hard disk using one passphrase. This is however in stark contrast with the situation for enterprises: especially in an environment where multiple parties must have access to the data, key management can be challenging as they must consider how trustworthy each party is and incorporate this knowledge into the key management structure. Second, in spite of advances in cryptography [70] it is still infeasible to perform extensive computations on encrypted data. Thus for any 
computation to be done, the data must be decrypted, which leaves the data exposed to attacks. As an example, PCI-DSS (the Payment Card Industry Data Security Standard) requires that credit card numbers are stored in encrypted form in a database [37]. However, it does not consider in detail how the decryption keys should be managed that are necessary for processing.

\section{Change Perimeter Placement}

Next to data-centric security, another option is to shift the placement of existing organizational network perimeters, First we will show options for making the set of systems that the organizational perimeter encloses smaller, and then we discuss options for perimeters around different organizations, or crossing organizational boundaries. The Jericho Forum calls these latter "macro perimeters" [109].

End Point Perimeters The end point security principle dictates that each device must be responsible for maintaining its own security. Forms of end point security are server and client firewalls, anti-virus software and intrusion detection systems on laptops. In case the network infrastructure is fragmented and communications between devices are ad-hoc, end point perimeters can be used to ensure security: the network does not need to contain any security mechanisms, it simply must be available. End points can then simply encrypt their communication using Virtual Private Network (VPN) technology or transport layer security (TLS). Ideally, each end point is a very simple system that is easy to secure. Unfortunately, an end point is often a complex system, for example a laptop or tablet with dozens of installed programs that all require Internet access. In such a situation it can be difficult to secure it adequately. In any case, end point security can also be a single point of failure: if the security of one device is breached there is no fallback security mechanism.

Zoning Some have argued that security can only be achieved by partitioning an organization's information systems into different zones, each having different security levels, allowing for a defense in-depth. Typically, the inner-most part is the best secured part. In situations where services are outsourced, this model can be extended to include an extended security perimeter [221]. The reason for using multi-layer security is that there are distinct parts of a system or organization that need to be better secured than others; while the parts that surround it are also under the control of the organization. As such, it allows for defense-in-depth. The drawback of zones is that their design can become very complicated, especially when a layer has to be partitioned itself, for example because each business partner requires its own environment. Furthermore, the classification of which system is most important is a challenge in itself. 
Cloud Perimeters With the rise of cloud computing, a new option are perimeters around clouds. The cloud provides a form of security-as-a-service. For example, cloud service provider Amazon has a tight control over its own infrastructure and can offer a strictly regulated working environment inside its own cloud, which is shared by multiple customers at the same time. A disadvantage of clouds is that they can also be used as resources by criminals. So far, clouds have been used for activities such as cracking passwords, controlling botnets and delivering malware [130]. Strong separation between processes of different customers is required to prevent criminals from accessing customer data.

Country Perimeters Nations also are involved in the creation of their own perimeters. For example China has a extensive firewall system around its systems [45]. The United States is also examining options, for example to implement an Internet kill switch in case of foreign attacks [121]. Concerning the effectiveness, these country perimeters can offer limited protection against attacks. A potential ethical issue is whether such perimeters are desirable, as they can impede the free flow of information (and thus of free speech).

ISP Perimeters Internet service providers can also create perimeters by filtering traffic [159]. They can filter out malicious traffic for their customers, or remove viruses from emails. ISP perimeters are easier to implement and control than country perimeters, as users of ISPs typically have a control panel to enable or disable certain security features. Drawbacks again include the option to limit the free flow of information.

Internet Perimeter Security A more radical (and currently only hypothetical) approach is to put a perimeter around the entire Internet. Such an approach is foreseen by Lessig [129]. "Ideally", Internet access is restricted to those systems and individuals that have identified themselves properly. With a functioning identity layer, trust becomes easier to organize, as organizations do not have to build the trust network themselves. There have been a number of initiatives that would support such an architecture but so far, attempts to create even limited forms of identity layers (for example Microsoft Passport [135]) have failed. The drawbacks are also clear: the entire infrastructure of the Internet would need to be changed, and privacy would be lost if everyone and every device is identified.

\subsubsection{Legal Domain}

In the previous section, we discussed technical solutions to vulnerabilities created by de-perimeterization, and their applications and limitations. These are not the only mechanisms for re-perimeterization: because de-perimeterization is a 
phenomenon that involves blurring boundaries between organizations, the solutions can also involve the restructuring of the perimeters between organizations, and between organizations and individuals.

\section{Organizational Re-perimeterization}

With organization re-perimeterization, we try to reverse de-perimeterization. For example, when the management of applications was outsourced, it can be insourced again, and placed under control of the organization. The main advantage of this approach is that it reduces the number of legal parties involved. This perimeter type is typically used in financial institutions and governments. An important motivation for this approach is that certain risks are simply unacceptable and must always be prevented, and cost is less of an issue. For example an intelligence agency cannot outsource its data center and have it hosted in a foreign country. Organizational re-perimeterization can be done on the condition that the organization has control over its network and has the available expertise to maintain it. The largest problems with this solution are cost and lost business opportunities: it is very difficult to manage applications properly; it requires highly trained and expensive personnel.

\section{Individual Perimeters}

An alternative to organization level security is to make every individual responsible for keeping herself and her own devices secure. A system uses "individual-centric" security if the burden of maintaining security is put down on individual users. If a person works on her own laptop on her own project, on her own document, in her own free time, there is no organizational security perimeter needed and de-perimeterization cannot occur.

Examples of individual-centric security are the usage of individual laptops and mobile phones: rather than having a uniform set of desktops in place, where everyone can login at any system, organizations are now shifting towards a concept where users all have their own dedicated devices. Some systems that store medical information also use individual-centric solutions, for example Microsoft Healthvault [134] or the now defunct Google Health [74]. Here individuals can manage their own medical information and decide themselves who will get access to these records (for example which doctor or which family member).

The concept is not always applicable as it can be at odds with organizational alignment. Organizations are a natural way to structure economic activity (Cf. Coase [34]) and not every transaction occurs between individuals, or can be designed in such a way. In the aforementioned healthcare domain, a medical file could be controlled by one patient, but she still has to give some authorizations to a hospital, because she cannot authorize all nurses individually. The need for 
organizational alignment is clearly visible in individual-centric systems such as the online social network Facebook, where "business accounts" appear. For example, Warner Bros, a multi-billion dollar company, has its own "user" account on YouTube called "warnerbrosrecords". This leads to the conceptual problem of which employee (or employees) of Warner Bros has access to this account, and whether user rights are managed by either Warner Bros or by YouTube. A similar problem is currently occuring on the microblog service of Twitter, where "users" are actually company employees who have full access to a corporate account, without any restrictions imposed on them. In these cases, de-perimeterization does occur and individual perimeters cannot solve the problem.

A final issue is that users can be incapable of securing their systems. This limits corporations tolerance for allowing employees to use their own devices, the so-called Bring-Your-Own Device (BYOD) phenomenon [6]. It is already hard for a healthy individual to maintain her own laptop, install patches and configure the firewall, but it is even harder for an ill person to manage the authorizations to her medical file. One approach to deal with user-owned devices is virtualization. The phone then runs two separate virtual machines, one for accessing personal data and one for accessing corporate systems, the latter being managed by the corporation itself [13].

\section{Virtual Organization Perimeters}

Concerning organizational perimeters, we can also create new organizational structures, or so called virtual organizations [139], entities comprised of members of other organizations that work together on goals important to each participating organization. (In the context of cloud computing, this is called a "community cloud" [131]) These effectively reduce the number of connections between different legal entities. Virtual organizations are extensively used in grid computing, where separate institutions use resources from yet other organizations or even individuals. By combining the institutions into one virtual organization, the resource owners only need to deal with one entity. Another typical case is in outsourcing relations, where a separate unit is created to monitor a service-level agreement. On the downside, virtual organizations require trust in the other parties and a virtual organization is still yet another organization, and can thus also introduce new complexity, because employees become a member of two organizations rather than just one.

\section{Federated Perimeters}

A final legal solution direction is to use federated systems [68]. If security cannot be addressed on the organizational level, maybe it could be implemented at the inter-organizational level, by putting a security perimeter around multiple 
organizations. In a federated solution, several organizations agree to use a shared security solution, the features of which can be used by all participating organizations. Federated systems can especially help with identification and authorization. If someone is identified at one organization, she can use his credentials at another organization. Just as with virtual organizations, the advantage of federated security is that the number of connections between organizations can be reduced drastically. An example of a solution that supports federation is Eduroam, a roaming service that allows users from higher education institutions to use each others' Internet connections ${ }^{6}$ For federations to work, multiple organizations must come to some form of agreement and sometimes legal problems prevent information sharing.

\subsubsection{Physical Domain}

Underlying the previously proposed treatments were the assumptions that by applying existing digital security mechanisms, or rearranging organizational structures, we can reduce the problems of de-perimeterization. However, as mentioned in Section 3.3. de-perimeterization is the replacement of physical by digital connections. Thus, outside of the legal and digital domain solutions might exist in another domain, namely in the physical domain. Information technology has both benefits and deficiencies, and after decades of automation, we can assume that some of these are innate: a business process realized in IT fundamentally differs from a process realized in the physical world. Taking this view, the question arises if a re-introduction of physical processes can improve information security. There are three conceptual strategies for this re-introduction.

\section{De-automation}

First is a defensive strategy that we call "de-automation" where the emphasis is on reducing the harmful impacts of IT. Such a strategy might be expensive from a business opportunities point of view, but in some cases the loss of business opportunity is outweighed by the gain in security.

One specific case where business processes could not be realized easily by IT is democratic voting. After adopting electronic voting systems, both the Netherlands and Germany banned these machines for being insecure, finding that the opportunities of e-voting (such as timely results) did not outweigh the threats to democracy (fraud and violations of vote secrecy). Thus they switched back to a physical, paper-based approach [228].

6wW . eduroam.org 


\section{Recombination}

Second is an offensive strategy that we call "recombination", where the objective is to combine physical and digital mechanisms to improve information security, rather then discontinuing the use of IT.

However, combinations are not always more secure. We observe that digital systems are actually becoming more intertwined with the physical environment, and this actually creates new security threats. For example, smartphones and tablets are becoming increasingly resourceful, and use motion sensors and cameras to observe their environment. These systems thus threaten existing security mechanisms by combining physical and digital attacks in new ways. A smartphone with camera can be used to identify the password as it is typed by a user on the screen of another device [133]. RFID chips allow information processing capacities to be embedded into everything physical, leading to a whole new range of privacy threats, as they can be used to track physical items [63].

\section{Simulation}

Third is a strategy that we call "simulation". Rather than de-automatic IT systems, we can simulate the positive aspects of physical security, while we can reduce the negative consequences of de-automation. For example if we are concerned about data leakage there is no need to go back to paper: instead we can reduce the transmission speed of our systems so that no more than a couple of pages of text can be transfered at a time. The obvious drawback of this approach is that such simulations are less "hard" than real physical security. A hacker would be able to break them more easily.

\subsection{Conclusion}

We derived the essential characteristics of the de-perimeterization trend. In our view, this trend is not linear but cyclic, and entails the replacement of physical by digital connections. Next, we listed several treatments for it. We found that data-centric security, one of the cornerstones of the Jericho Forum's solution called the collaboration-oriented architecture, suffers from several problems. Although it can be a partial solution for security problems, it cannot be the logical conclusion of de-perimeterization.

Can we actually re-engineer ourselves out of the current-day security problems with legal or digital solutions? Obviously, de-perimeterization is brought about by IT, as it played a crucial role in the case of both Wikileaks and Stuxnet. Without IT it would never have been possible to share so many documents so easily with so many people, or attack a nuclear facility remotely. Maybe the lesson from the Wikileaks case is that secure and digital sharing of so many secrets is 
impossible. and - benefits set aside - they would have been more secure printed on paper and kept in a vault.

To improve system security, we should therefore understand the different capabilities of both physical and digital systems such that we know how to combine them securely and how to perform trade-off analysis between security and business opportunity. This knowledge is even more important because digital systems are merging more and more with their physical environment. In the remaining chapters we will try to build up this knowledge about physical and digital security. 


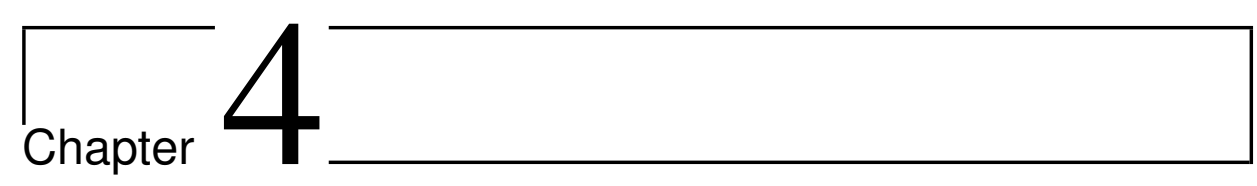

\section{Related Work}

In this chapter we will discuss related work about physical and digital security. The purpose is to investigate what is already know about this topic and to find approaches that can be used for our own research. First we examine broad-range theories about the effects of automation and security. Next we consider integrated security mechanisms, IT architecture frameworks and formal models about physical and digital security. Once this information is gathered we can perform a gap analysis: what knowledge is missing to answer our research questions?

\subsection{Broad-Range Theories}

Sieghart was one of the first researchers to consider the security consequences of automation. In 1976 he noted a potentially negative impact of automation on privacy. [194]

More transactions will tend to be recorded; the records will tend to be kept longer; information will tend to be given to more people; more data will tend to be transmitted over public communications channels; fewer people will know what is happening to the data; the data will tend to be more easily accessible; and data can be manipulated, combined, correlated, associated and analyzed to yield information which could not have been obtained without the use of computers.

Expressed in terms of the security properties of Chapter 2, Sieghart claims that IT causes a decrease in the confidentiality of information and an increase in availability.

For Floridi [58], the starting point for understanding the impact of IT is the "2P2Q hypothesis", which encompasses widely held beliefs. First, automation 
make it easier to process data at a faster pace (the two P"s), and second it increases the quantity and quality of that data (the 2 Q"s). Thus, IT leads to increased integrity (quality), availability (quantity) at the expense of privacy and thus of confidentiality.

This view is not universally valid as there are exceptions to it; some specific tasks are not faster in IT and not everything can be done better by computers. For example human vision is far superior to digital cameras in daily life. Furthermore the "pace" of computers makes them also susceptible to making mistakes at a much larger scale than persons. Floridi too argues that IT causes both a decrease and an increase in informational privacy. One specific way in which automation reduces privacy is because it reduces the difference between processors and the processed: digital programs can easily deal with digital data, whereas physical manipulations are more cumbersome. Alternatively, IT offers the opportunity for privacy enhancing technologies as well.

Yet another viewpoint is given by Blakley, who sees physical and digital security (not only privacy) as a difference between inherent and imposed properties [21]. Physical systems have inherent properties, whereas logical systems have imposed properties. He gives the example of cash: in physical form it is hard to steal millions of dollars because their combined weight and size make them hard to carry. By contrast, theft in digital form can mean as little as swapping several bits.

Thus security in the digital domain is "inherently" more brittle than in the physical domain. Blakley further argues that integrity and confidentiality are hard to achieve. First integrity requires almost perfect security itself, as any security gap threatens it. Second it has been demonstrated that keys (both passwords and cryptographic) are hard to keep secret.

From the legal domain, Lessig discusses digital rights management systems in his book CodeV2, and how they evolved after technology made it easier to copy [129]. One specific effect of automation is easy copyability. The actual effect of IT depends on laws and policies, which can be codified in software and run at little effort. For example, code allows corporations and governments to monitor and restrict actions of citizens. Thus, Lessig also observes an increase in availability and integrity and a reduction of confidentiality and privacy. 1

Analysis The aforementioned theories point in the general direction of increased availability and reduced confidentiality. Although the theories are backed up by evidence (IT causes privacy violations), the mechanisms that lead to increased availability and decreased confidentiality are not explained clearly by them, and the possibility remains (motivated later in this thesis) that other outcomes are possible with these mechanisms or that other mechanisms might exist. Concerning

\footnotetext{
${ }^{1}$ Cf. Chapter 9 for a more detailed explanation of Lessig's theories.
} 
the difference between inherent physical and imposed digital properties that Blakley observed, the same information can also be used to conclude that there actually are specific properties of digital systems, namely that they have the inherent property of malleability, which is not always a desirable property.

\subsection{Integrated Security Mechanisms}

Location-based Access Control Denning and MacDoran (1996) advocated the "anchoring" of IT systems to the physical environment. They see IT systems as too volatile and they must be "grounded" into physical processes, forcing them to go through a specific physical location or device [47]. One specific example of grounding is Location-Based Access Control (LBAC). This is a form of access control that takes the user's physical location as input for its access control decisions. For example, an LBAC system can ensure that a user only gets access to a document if she is physically present in a certain office (ignoring any vulnerabilities that might be present in this system).

Physical Captchas To make sure a physical person is in the loop, captchas are a well-known method. Captchas are digital puzzles given to users to provide evidence that they are human, for example by identifying difficult-to-read letters in an image. Golle and Ducheneaut propose "physical captchas" as an extension [72]: tests that differentiate between humans and bots based on person's capabilities to interact with the physical world. For example to ensure that only real persons play in online games, users can be asked to perform physical actions on a device, such as moving fingers on a touch screen. Hardware that can mimic and automate such tasks is difficult and expensive to design and the device thus serves as a physical captcha. (Two requirements are that the device that delivers the output is tamper-proof and that the output itself is authenticated).

Server Virtualization Server virtualization, turning physical servers into virtual machines, brings along a new set of attacks and defenses [84], [118], [215]. Apart from scientific studies, virtualization software vendors such as VMware have also written extensively on virtualization security, guiding enterprises on how to perform infrastructure hardening [216] and server configuration [219]. A general advice is that virtual machines should be secured as physical machines, and be equipped with intrusion detection systems and anti-virus. To further improve security, Perez et al., combine virtualization with hardware-based Trusted Platform Module (TPM) chips [166]. They use the TPM chip in two ways. First, the TPM can check the security status of a virtual machine, serving as a root of trust. Second, virtual machines can also invoke the TPM to run security checks. 
For this the TPM chip itself must be virtualized (meaning: shared between different virtual machines), resulting in a complicated mixture of physical and digital security.

Analysis Obviously, the usefulness of location-based access control depends on the environment in which the users are present, but researchers do not clearly explain the role of the environment and what the benefits are over normal logical access control [33]. As for physical captchas, it is not clear how much security is actually increased, compared to logical security mechanisms.

If we consider virtualization security, the advice to employ the same security mechanisms for a virtual machine as for a physical machine is sound if we consider that their value (consisting of the live processes involved in business processes and the data stored on it) is similar. However at a more conceptual level, a VM cannot be secured in the same way, because there are differences between physical and logical security. The threats are different and we must understand what physical properties are important to security and how they differ from logical properties.

\subsection{IT Architecture Frameworks}

Enterprise Architecture Design Normal business process design methodologies abstract from the physical world and place an emphasis on the digital domain. If we consider any design method for enterprise architecture (the structure of an enterprise and the relationships of units inside the enterprise) we would find a common division of domains [77]:

1. business processes and activities

2. applications that implement business processes and process information

3. technology that supports the applications

Designing an enterprise is seen as a top-down process where the emphasis is on realizing goals with IT. First we design a conceptual business process, second we select and configure the applications to execute it, and third we consider how to implement it technically (and physically).

Cyber-Physical System Design In contrast to enterprise architecture design that was discussed in the previous paragraph, the physical domain is of much more importance for the design of cyber-physical systems (CPS), which regulate physical processes, such as engines in cars or industrial manufacturing processes. CPS are specifically designed to interact with and/or control the physical environment. Security challenges are discussed in the context of cyber-physical system 
research [4], [123]. Security problems include privacy, as CPS typically gather data from sensors that can be traced back to individual users, the availability of network communications (risk of jamming) and trust issues. It is necessary to understand how to compose CPS, taking into account their physical and computational properties. [150] Concerning security, Cárdenas et al. investigate points of attack in a typical CPS, consisting of a physical system and a controller with feedback loop. [26] Partial solution designs are proposed by Zimmer at al. [232], who propose time-based intrusion detection for CPS, especially for embedded systems.

Analysis The aforementioned enterprise design method is only rational if the lower levels can indeed realize the designed business processes securely. However, it is also common knowledge that security is an aspect (a cross-cutting concern that is hard to isolate) and therefore security should be an integral part of system design and is difficult to implement afterwards [191]. If some security properties can only be realized in a specific physical way, this complicates the top-down approach. As for CPS, integrated design methodologies are lacking as well. Solutions are only partial and realized in the digital domain, and do not actually combine physical and digital security mechanisms.

\subsection{Formal Models and Simulations}

Models A first body of related work on physical security concerns modeling procedures that span across the physical, digital and social domain. In these models security is determined by all these domains: physical and digital security are mutually dependent. Probst et al. [168] and Dimkov et al. [48] have developed such models. Threats can span different domains, for example an employee receives an USB stick from a friend (social domain), and plugs it into a computer (physical domain), causing a security breach in the server (digital domain). The models allow formal verification of certain security characteristics, to determine if certain attacks are possible, taking into account existing security policies.

Weldemariam and Villafiorita propose a method for analyzing procedural security [225]. Procedures are actions executed by agents on assets that can belong to both the digital and the physical domain. To this effect, they create UML activity models to represent procedures and describe possible actions on assets, either digital or physical. They define threat actions such as replacement and removal on these assets. Next, they extend the model with threats and asset flows and define the security objectives. A model checking approach is used to assess the security of the procedures.

In a similar approach, Bryl et al. evaluate procedural alternatives concerning the introduction of e-voting [23]. They combine process modeling in UML (use case, activity and object diagrams) with goal-driven reasoning in the agent- 
oriented modeling tool Tropos. In particular, UML is used to model both existing "as is" and proposed "to be" processes, and they use Tropos to reason about design alternatives and investigate security issues. Finally the Tropos model is transformed into a formal Datalog model to automatically verify model properties. If different models where security is realized in both digital and physical ways are available, the procedural analysis approach could help to perform physical and digital trade-off analysis.

Simulations A different approach to understand physical and digital security is to simulate systems. Rather than proving (im)possibilities, events with probabilities are simulated. This approach is proposed by Pardue et al. in the context of e-voting. They advocate using simulation methods to determine the security of voting systems [160], both electronic and paper-based. The first step is to create a threat tree: a hierarchical structure that expresses how (starting with the leaves of the three) a threat (the root node) can be realized. Nodes are connected by special AND nodes (all leaves must be realized) or OR nodes (one of the leaves must be realized). Experts then estimate the likelihood of the leaves (called TERMINAL nodes), which are tied to the motivation of attackers and the complexity of the attack itself. In turn, this information is used to run simulations with a Monte Carlo method, which is input for a risk assessment and trade-off analysis between specific systems, both paper and electronic.

Analysis There are two assumptions underlying the previous models and simulations. First, security is always a mixture of physical of digital security and second, improving security is possible by developing fine-grained representations of existing situations, and analyzing them for security weaknesses. Even though they are fine-grained, these representations are also abstractions and idealizations from the enormous complexity of physical processes. Whether these models are useful depends in part on the validity of the abstractions they make. However these key design decisions are not made explicit. We do not know from which properties we can safely abstract away while still deriving meaningful results, and which properties are important and must be expressed in the models.

\subsection{Summary}

In this chapter related work about the differences between physical and digital security is investigated. Literature concerning broad-range theories, integrated security mechanisms, IT architecture frameworks and formal models and simulations were all examined for knowledge about these differences. This investigation did not lead to clear detailed knowledge about these differences. In fact, our investigation leads us to conclude that the answers are so "obvious" that there 
is limited scientific literature on the subject. Knowledge about the differences between physical and digital security is only partially available, and is scattered across various branches of computer science. Integrated design methods and modeling tools are based on insufficient problem understanding and are therefore not sufficient for a good trade-off analysis. Thus, we are in need of more knowledge on this topic, and a method to derive this and perform trade-off analysis. This will method will be presented in the next chapter, where we explain our research approach. 
Related Work 


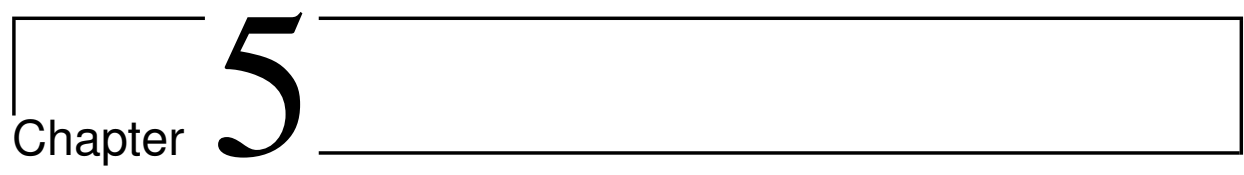

\section{Method}

\subsection{Introduction}

In the previous chapter we examined what is currently known about physical and digital security and concluded that more knowledge is required to make a trade-off analysis. We will now develop our research method for deriving this knowledge. The first section explains the overall research design, which is explained in more detail in the next sections. The chapter finishes with an investigation of the validity of this research design.

\subsection{Research Design}

Given the lack of usable information about the differences between physical and digital security found in the related work, we chose to take a "greenfield" approach and create theories bottom-up from case studies, discover heuristics and solution concepts. Such an approach requires the study of multiple cases of systems that involve physical and/or digital security, from which we can extract mechanisms at work. As such, our research design is that of a multiple case-study. A case study is defined here as the analysis of a class of systems as a whole. Analysis can be performed through a variety of methods including literature studies, interviews and reviews of primary documents.

There are two levels of case studies. At the highest level, there are four cases that each concern a specific business process, for example voting or rights management. Each of these case studies has its own chapter. Within each of those chapters, there are three sub-cases: physical, digital, and hybrid systems, which are implementation of the business process. First, we studied one or more cases of systems that were maximally physical. Second we examined cases where the 
systems were maximally automated. Third, we investigated cases that were for a large part both digital and physical. We call these hybrid systems.

In each sub case study, we extracted information about the threats that deperimeterization causes, the physical and digital means to mitigate those threats and the combinations that can be made. Between every two sub case studies, we performed a cross-case analysis (Cf. Eisenhardt [53] to understand the differences between physical and digital systems as well as their trade-offs. We compare:

1. Physical vs. Digital systems

2. Hybrid vs. Physical systems

3. Hybrid vs. Digital systems

Data from multiple high level cases is put together in a step-wise fashion, where an initial hypothesis is checked against all available data. If the hypothesis does not fit the new data it is changed, particularly by altering the conditions under which it holds true. This approach is similar to analytical induction [180] and is used for the case studies. Thus at the end of case study four, all knowledge developed from the case studies is codified.

After completion of all the case studies, this knowledge is used to construct two methods that can be used directly by practitioners:

1. Method for analyzing the security of existing systems.

We present a method for using the identified physical and digital properties to identify threats and vulnerabilities.

2. Method for designing new systems.

We present means to integrate physical and digital security mechanisms (the hybrid systems) as security patterns [51], based on the taxonomy developed in answer to (RQ4).

These results are then validated in a focus group meeting (Cf. Morgan [138]). Figure 5.1 illustrates the research design. In the next sections we will explain the research design in more detail.

\subsection{Case Selection}

A multiple case study design requires criteria for which cases to include and for deciding how many cases are needed to understand the differences between physical and digital security. First, as to what constitutes a case, we defined a case loosely as a class of systems used for the same purpose.

Cases were selected through theoretical sampling [145]: we selected heterogeneous cases in which trade-offs between digital and physical security mitigations have been made, to identify the recurring patterns in these trade-offs. This 


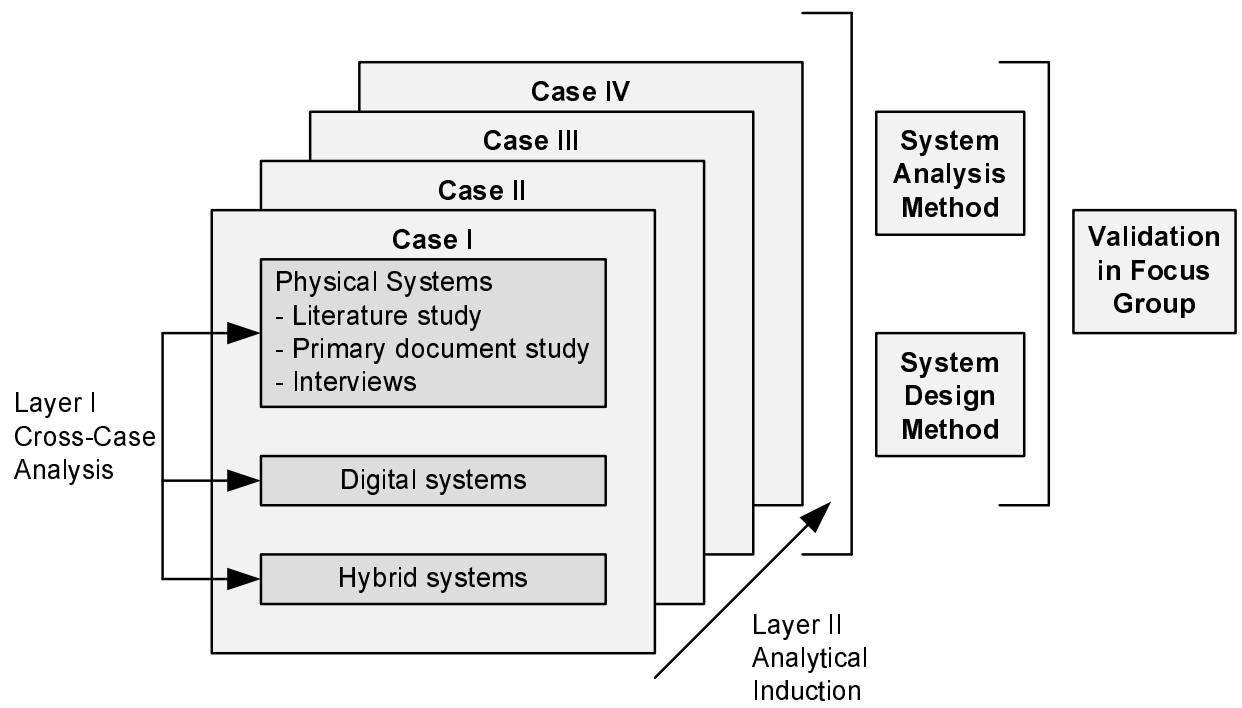

Figure 5.1: Research design with layered case studies.

enhances the generalizability of our findings. If a new case did not yield new patterns of trade-offs, this increased the support for the claim that we had found the most important patterns in these trade-offs that would occur in different cases where such trade-offs have to be made.

Case-based reasoning aims at identifying structures in a case that produced phenomena observed in the case, and that are likely to produce similar phenomena in similar cases. The structures we focused on concern the architecture of a case, meaning the composition of a case in terms of entities and their possible interactions, and the mechanisms of these interactions.

At the top level, we tried to apply the following criteria for selecting cases:

1. The systems should have different but equivalent physical and digital realizations, or consist of a combination of physical and digital components.

2. The systems should have known security problems or should be used to secure other systems themselves.

3. The classes of systems that we investigate should be heterogeneous, to achieve a good theoretical spread.

We will now examine our top-level cases in more detail. To begin, Figure 5.2 gives an overview of the cases in our study. This shows that criterion 1 is satisfied. Security problems of these systems (criterion 2) will be discussed in each individual case study chapter. As for criterion 3, this is discussed in Section 5.13 on Validity. 


\begin{tabular}{|c|c|c|c|c|}
\hline Case & Chapter & Physical & Digital & Hybrid \\
\hline Access control & 6 & $\begin{array}{l}\text { Locks and } \\
\text { doors }\end{array}$ & $\begin{array}{l}\text { Logical } \\
\text { access control }\end{array}$ & LBAC \\
\hline Voting & 7 & Paper voting & E-voting & $\begin{array}{l}\text { VVPAT } \\
\text { Vote printer } \\
\text { Optical scan }\end{array}$ \\
\hline IT infrastructure & 8 & $\begin{array}{l}\text { Physical } \\
\text { servers }\end{array}$ & $\begin{array}{l}\text { Virtual } \\
\text { servers }\end{array}$ & $\begin{array}{l}\text { Partial } \\
\text { virtualization }\end{array}$ \\
\hline Rights management & 9 & Paper books & Watermarking & $\begin{array}{l}\text { Software } \\
\text { activation }\end{array}$ \\
\hline
\end{tabular}

Figure 5.2: List of case studies.

\section{- Access Control Systems (Chapter 6)}

We examined means to control user access to digital and/or physical resources, and combinations thereof. Specific attention is given to a combination of logical and physical access control based on a user's location: Location-Based Access Control (LBAC).

\section{- Voting Systems (Chapter 7)}

We examined different means for democratic voting, including paper voting and electronic voting. Hybrid systems include those that allow the voter to check a printed audit trail (Voter-Verified Paper Audit Trail or VVPAT), those that print votes or that scan the votes optically.

\section{- IT Infrastructure (Chapter 8)}

We examined means to arrange IT infrastructures and examine the impact of virtualization technologies on security, which decouple applications from the underlying hardware. Hybrid systems include virtualized servers, hardware-enabled security mechanisms using Trusted Platform or Hardware Security Modules (TPMs, HSMs).

\section{- Rights Management Systems (Chapter 9)}

We examined systems that limit what end-users can do with content, such as movies and music. Such limitations are "automatically" realized in the physical domain, but are more difficult to replicate in the digital domain, where a wide range of Digital Rights Management (DRM) systems have been developed, for example to secure PDF files. Hybrid systems include game consoles and E-books that are only readable on specific E-readers. 


\subsection{System Representation}

In order to answer the research questions we need to represent physical and digital systems. We list what we view to be four core requirements for these representations. Any approach should minimally incorporate four elements:

E1: argument structures that can relate low-level system structures to business goals

E2: ability to reason about social and physical system context

E3: means to express violations of security goals

E4: means to express multi-step attacks

First, hierarchical arguments are necessary to indicate how, in complex enterprise systems, low-level system structures contribute to higher level business goals such as confidentiality. Second, contextual factors are required because systems achieve security in cooperation with the social and physical environment, and this dependency should be made explicit. Third, no security argument is complete without considering how attacks might violate security goals by bypassing security mechanisms. Fourth, attacks can take multiple steps: steal a smartcard, gain access to a webserver and copy sensitive data from a patent application database. Such steps must be represented as well.

Ideally, such an approach should be formal, to allow security proofs, simulations and probabilistic risk management. However, formalization of social, digital and physical structures requires abstractions and assumptions that are at least partly motivated by the capabilities and limitations of the formal system. Our goal in this thesis is to avoid such abstractions and assumptions, and be able to express properties of the physical, digital and social worlds, that are relevant for security trade-offs, without being limited by the properties of a specific formal reasoning system. Below we explore several generic methods that can realize one ore more of these requirements and assess their capabilities in more detail.

\subsubsection{Toulmin Arguments}

One means of reasoning is through so-called Toulmin arguments [146]. A Toulmin argument begins with a specific claim, which is supported on certain grounds, preceded by a modal qualifier (always, possibly) about the strength of the claim. A warrant provides further arguments about the support of the grounds to the claim. In turn warrants themselves can have backing. Finally rebuttals describe what could invalidate the claim. Toulmin arguments have been used for security requirements engineering by Haley et al. [80] By constructing Toulmin arguments, architects can investigate the security of a system, hypothesizing about 
potential threats (rebuttals) and mitigations (new grounds or warrants) until they have assessed the security in sufficient detail. Figure 5.3 shows the structure of a Toulmin argument 1

Note that Toulmin arguments are static structures and do not represent sequential steps that an attacker or defender executes. A potential workaround is to represent attack steps as rebuttals and mitigations as new grounds or warrants in a long chain, but this representation can easily become very complicated.

Toulmin arguments thus satisfy the requirement E1 concerning the hierarchical structures and $\mathbf{E 3}$, the means for expressing the violation of goals. Toulmin arguments do not have an explicit way to reason about the social and physical context $\mathbf{E} 2$ and neither can they properly express multi-step attacks $\mathbf{E} 4$.

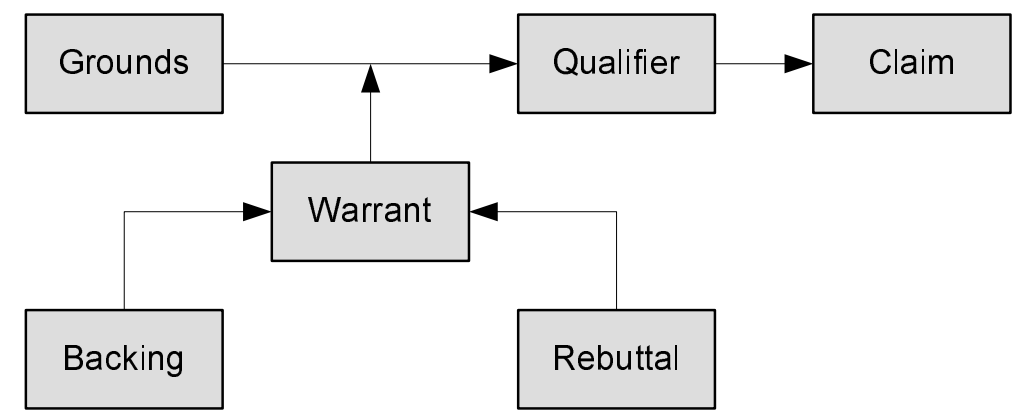

Figure 5.3: Schematic overview of a Toulmin argument.

\subsubsection{The Systems Engineering Argument}

Systems do not realize security goals in a vacuum. Rather, security is realized in collaboration with the environment on which it depends. The logical expression for this is the system engineering argument:

a system in a certain context that satisfies assumptions will produce emergent properties [2], [226].

If the emergent properties are desired, we can also designate them as goals. Thus, when designing systems, it is important to understand the assumptions that are made about the context. (Cf. Section 1.4) Thus, the system engineering argument offers a means to consider the context in which physical or digital security is most effective and why this is the case. Systems engineering thus satisfies requirement $\mathbf{E} 2$ but not the other requirements.

${ }^{1}$ There are various ways in which a rebuttal can be connected to an argument [146]. We describe it here as the conditions under which a warrant no longer holds. 


\subsubsection{Attack Trees}

In the previous sections we examined approaches for understanding the impact of a system, by examining its structures and the contributions of the environment or, in the case of Toulmin arguments, examining reasons (rebuttals) why it would be insecure. One potential rebuttal is an attack, but the problem is that such attacks can require a series of steps, which are not originally represented in Toulmin arguments. However, they can be represented with attack trees. Attack trees are diagrams that model the various ways in which an attack can be realized [132]. The final step in the attack is the root node, whereas the child nodes contribute directly to realizing their parents.

Attack trees can thus directly represent cause and effect relations necessary for our analysis ( $\mathbf{R 4})$ but cannot satisfy the other requirements.

\subsubsection{KAOS}

The KAOS requirements engineering method [214] satisfies all our requirements as follows: concerning the context (E2), KAOS has the notion of domain properties, characteristics of the environment such as invariants (for example physical laws) or hypotheses that contribute to goal realization. As for the hierarchical structure (E1) and chains of events (R4), a goal is achieved in KAOS if all its subgoals are achieved. Decomposition of a goal into subgoals is possible on various grounds, among those definitions (for example: security is defined as confidentiality, integrity and availability) but also process steps that need to take place to reach the parent goal. Typically the higher level goal decompositions are based on definitions, while lower level goals involve particular operations taken in a certain order. Threats can also be expressed in KAOS, and thus it satisfies also $(\mathbf{E 3})$. This makes KAOS the only approach that satisfies all the requirements and it is thus the method we will use in the case studies. KAOS concepts are defined in Figure 5.4

A KAOS requirements model starts from goals, the objectives that need to be achieved. Goals can be decomposed into subgoals, resulting in a tree-structure $2^{2}$ By definition, a goal is achieved if either all subgoals are achieved (AND decomposition) or one subgoal is achieved ( $O R$ decomposition). In some cases, goal realization also depends on the aid of the environment, which has certain essential characteristics called domain properties. Goals conflict when the achievement of one goal makes the realization of another goal harder. Obstacles hinder the realization of goals and can be resolved by setting other goals that prevent obstacles from occurring, which is called resolution. (In security terminology, the term threat is somewhat similar to obstacle, and the term mitigation to resolution.) Finally, agents execute operations on entities that contribute to the realization of the

\footnotetext{
${ }^{2}$ For brevity, we will not elaborate on the distinction between KAOS goals and requirements.
} 


\begin{tabular}{|c|c|}
\hline KAOS term & Explanation \\
\hline Object & Thing of interest in a composite system. \\
\hline Entity & Identifiable and independent object. \\
\hline Agent & $\begin{array}{l}\text { Active participant in a process (an agent is a special type of } \\
\text { entity). }\end{array}$ \\
\hline Conflict & $\begin{array}{l}\text { Situation when the realization of one goal hinders another } \\
\text { goal's realization. }\end{array}$ \\
\hline Domain Property & $\begin{array}{l}\text { Descriptive assertion about objects in the environment of the } \\
\text { system. }\end{array}$ \\
\hline Goal & Objective to be met by cooperation of agents. \\
\hline Obstacle & $\begin{array}{l}\text { Condition (other than a goal) of which satisfaction may prevent } \\
\text { another goal. }\end{array}$ \\
\hline Operation & State transitions of objects performed by agents. \\
\hline
\end{tabular}

Figure 5.4: KAOS terminology with explanations (adapted from Van Lamsweerde [214]).

goals. For future reference, we here present four elements of KAOS that we will use in several case studies in this thesis.

(i) $\{$ Subgoals, DomainProperties $\} \models$ Goal

This model expresses the role of the context (domain properties) in realizing security goals. For example in the physical world, the secrecy of the vote in a voting process is realized by the ability of the paper ballot to be folded (which is a domain property).

Actors (persons, software programs) execute operations that contribute to the realization of the goals:

(ii) $\{$ Specifications $($ Operations $)\} \models$ Goal

This model expresses that operations should be specified and contribute to the realization of goals. We can use this in two ways, first top-down to check whether all goals are operationalized, and second bottom-up to investigate which operations contribute to which goals. For example, in a voting process we can first investigate to what goals the folding of a paper ballot contributes. Second, we can list all security goals and find out if our specification of operations is precise enough to realizes these goals.

KAOS has two representations of negative impacts on goal realization. First, goals can conflict, when "under some boundary condition the goals cannot be achieved altogether" [174, p.46]. Second, obstacles can threaten the realization of goals. Obstacles can be resolved by setting goals that prevent them from taking place, which is called resolution. For a given goal, the list of obstacles is complete when their absence, together with the domain properties, realizes the goal: 
(iii) $\{\neg$ Obstacles, DomainProperties $\} \models$ Goal

We use this model to search for possible ways in which obstacles can be prevented and thus to investigate how security goals are realized. For example in a voting process we can search for ways to prevent the stealing of ballots.

An obstacle is simply the negation of a subgoal:

(iv) $\{\neg \neg$ Subgoals, DomainProperties $\} \models$ Goal

This property gives insight into the structure of the KAOS tree as every negation of a goal is a potential obstacle. We can use this model to search for obstacles: if in a voting process the secrecy of the vote is a goal, then we can search for obstacles that threaten this secrecy, such that we can include them in the KAOS tree. Thus, having shown how KAOS can fulfill the requirements listed above, we choose the KAOS requirements engineering method for our own research.

\subsection{System Data Collection}

Data collection within each (sub-)case was done using literature studies, but also included the study of primary documents, and interviews on-site. Regarding literature studies, we based our approach on the method described by Webster and Watson [223]. Here, literature is retrieved from well-known sources such as leading journals and additional literature is found by tracing back the cited papers and forward toward other journal papers, as well as more recent conference papers. Literature studies are used in two ways: first they are a source of background knowledge on the case study. Second, they are one of the methods for gathering data about the case itself. For example, we gathered data about electronic voting through a literature study. In addition, we studied primary documents, which are documents such as audit reports used in the primary processes being studied. Finally we interviewed stakeholders in organizations that contained the systems or processes studied in the case. For example, we interviewed an employee of the municipality of Enschede for the case study on voting, and a system administrator for the case study on virtualization of IT infrastructure.

Regarding the differences between security policies and mechanisms discussed in Chapter 2, we chose to focus on low-level "how" mechanisms, the building blocks that help to realize higher level policies and implementations. These mechanisms do not necessarily have to be specified by system architects: they can be implicitly assumed. For example, as we will see in Chapter7, the inertness of paper plays an important role in paper voting, even though the inertness mechanism is not explicitly addresses in many voting regulations. It is the objective of this thesis to discover such mechanisms, and we will thus not focus on high level policies: we construct KAOS models of low-level operations and explain how they relate to security goals. Exceptions can be made for low-level policies that are 
directly connected to mechanisms, such as in the case of location-based access control (Chapter 6).

The findings are presented concept-centric, meaning that all literature on a certain concept is discussed in one section. Further detail about which data was collected can be found in the method sections of the case studies (Sections $6.3|7.3| 8.3$, and 9.3 .

\subsection{Outline for Each Case Study}

Having presented the overall research design and the cases to be examined, we will now zoom in to the outline for each individual case study. This is shown in Figure 5.5 .

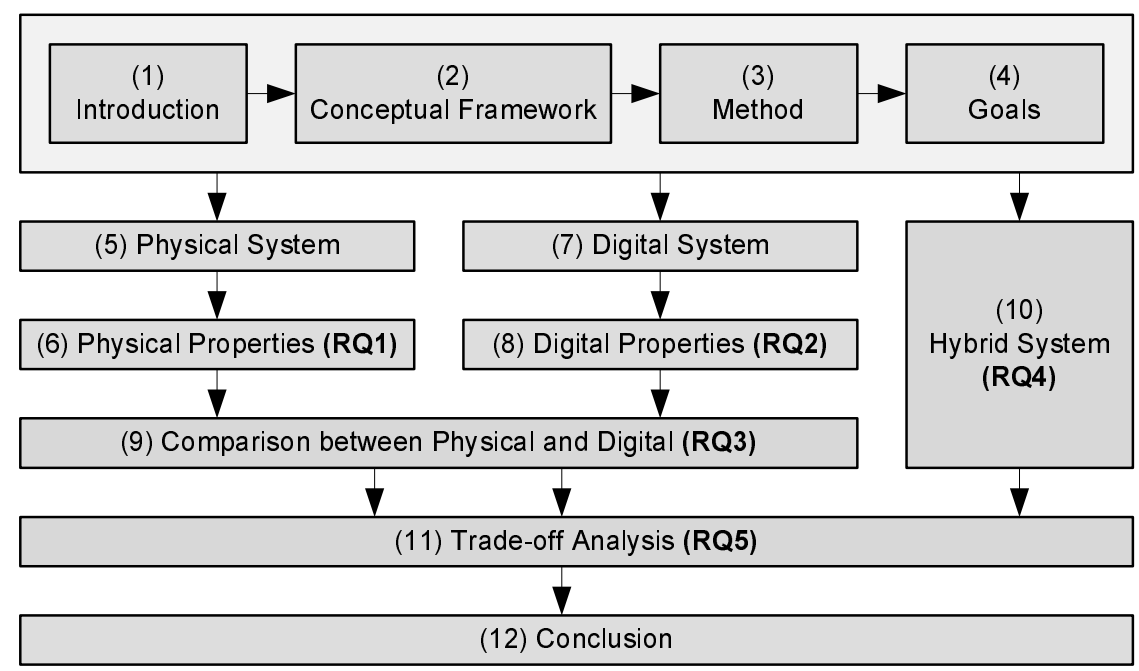

Figure 5.5: Outline for each case study. Numbers indicate the sections.

For readability, we do not present the output (the KAOS tree) as a whole. Instead we structure each case chapter as a running example and present parts of the tree when needed, throughout the chapter (even though it actually is a case study). Every case begins with an introduction of the topic and an investigation of the related work $(1,2)$. Next we discuss how we performed the research in the method section (3). We begin to create the KAOS model starting with goals, which are independent of the realization (physical, digital or hybrid) (4). Having identified the goals, we create the first KAOS model for a given physical system (5) and elicit the properties of physical entities (RQ1) (6). Next we examine a particular digital system (7), elicit the properties of digital entities (RQ2) (8) and compare this to the physical system (RQ3) (9). The final system to be researched is given hybrid system (10), after which we elicit different types of combinations 
of physical and digital security (RQ4). Finally we perform the trade-off analysis between physical and digital mechanisms (RQ5) (11).

Figure 5.6 shows in which parts we build the KAOS graph in most of the case studies, and Figure 5.7 shows how these parts connect to each other in a small example KAOS tree.

\begin{tabular}{lll}
\hline Part & Representation & Object Types \\
\hline Goals & Tree & Goals, Goal conflicts \\
Entities & Table & Entities \\
Operations & Table & Operation, Goal, Agent, Entities \\
Threats & Table & Threat, Goal \\
Properties & Table & Entity, Property, Threat \\
\hline
\end{tabular}

Figure 5.6: Parts used in constructing the KAOS tree.

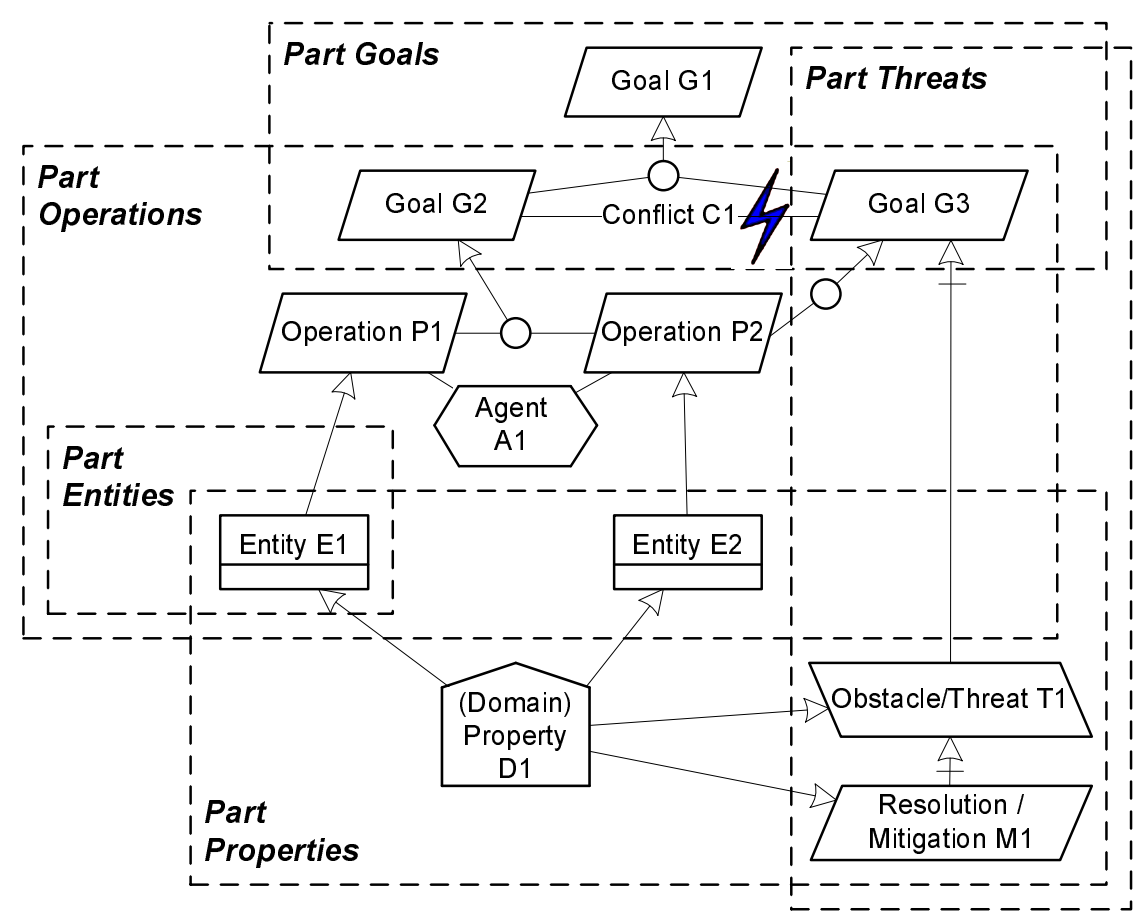

Figure 5.7: Example KAOS tree, showing individual parts of its construction.

We thus split the tree into five parts: to begin, we present the goals, where we show the relations between different goals, as well as goal conflicts. Next, we list the entities. After that, we show a list of operations, to which goals they contribute, who executes them (the agent) and what entities are involved. We 
continue with a list threats to the goals. Finally, we show how properties of entities contribute to goal realization (or the mitigation of threats to these goals).

\subsection{Analysis of Multiple Cases}

Combining data from multiple case studies can be done in two ways:

- by analyzing and integrating all data after completion of all cases

- by analyzing and integrating data after each case study

The first approach is typical for a cross-case analysis (Cf. Eisenhardt [53]). Here cases are compared to find similarities and differences between them. For similar cases, we examine differences, whereas for different cases we attempt to discover similarities. From this analysis arise categories, concepts and constructs, which after a series of iterations are further refined.

The second approach is taken by analytical induction (Cf. Robinson [180]). This methods concerns the study of a phenomenon and the conditions under which it occurs. The researcher starts with an initial hypothesis and then checks that information against the data that is available. When new data does not support the hypothesis two approaches can be taken:

- Redefine phenomenon.

This can mean the exclusion of cases, such that the hypothesis holds for a smaller set of situations.

- Modify existing hypothesis.

Change the hypothesis in such a way that it fits all the evidence.

At the highest level, we choose to perform analytical induction rather than cross-case analysis afterward. The reason is that cross-case analysis is most suitable for a situation where the phenomenon is not well understood in terms of concepts and categories and hypothesis: these are developed during the crosscase analysis. However, in this research, the structure of the hypotheses that is developed is already clear at the beginning of the research: namely a physical or digital property has a positive or negative effect on information security. (See Section 5.8.3.) Analytical induction allows us to take full advantage of this, by testing these hypothesis directly and in detail. We will however deviate from the normal approach of analytical induction in two ways:

- We do not a-priori select cases in which a phenomenon has occurred. (See Section 5.3.) Because the cases are selected from the entire population rather than from a subset this improves the external validity. 
- We do not start with an initial hypothesis; rather we create the first hypotheses after the first case study. The reason is that, as the related work shows, there are few theories about physical and digital security that can serve as a source for the initial hypotheses.

Thus, rather than presenting a cross case analysis at the end, we will therefore analyze the data case after each case, and check whether previous hypotheses (impact of physical properties on security goals, differences between physical and digital security and hybrid types) fit the data of the new case.

\subsection{Properties}

In this section we will explain how properties of physical and digital entities are derived. As for the goal, we stress that this is not to create a complete ontology of properties. Instead, the objective is more limited, namely identifying recurring characteristics of entities in order to understand more about how physical and digital security works. We will now begin to explain what we define as properties.

\subsubsection{Characteristics of Properties}

As mentioned in Section 5.4.4 KAOS defined domain properties as descriptive assertion about objects in the environment of the system. In this section we will further refine this definition, such that the analysis will yield structured results: properties are characteristics of entities that are represented in the form of adjectives.

For physical security, examples are "movable", "visible", "identifiable", "inert". Physical properties relevant for security relate to capabilities of persons: an object is "movable" because a person can move it. Likewise, an object is "identifiable" because a person has the capability to identify it. We chose to focus on the properties of physical and digital entities, and not on the capabilities of persons to observe these properties. Therefore we do not say that a person has the property "capable to see" but rather that an object is "visible".

For digital security, similarly to the way in which physical properties are tied to physical objects in the physical domain, digital properties are tied to the digital domain, which has conceptual entities such as files, data, processes, and network packets. Thus, even though identifying entities in the digital domain is less straightforward than in the physical domain, it is possible to understand specific characteristics that entities in it exhibit $3^{3}$ Concerning the method of identifying and defining digital properties, we must point out that often characteristics of the

\footnotetext{
${ }^{3}$ We start with the assumption that there actually are such digital properties - and take a different viewpoint than Blakley, who argued that digital systems are so malleable that they don't have fixed ways of behaving: in our view malleability could be a digital property as well.
} 
digital domain (RQ2) are actually described in terms of the differences (RQ3) between the digital and the physical domain. For example Floridi's 2P2Q hypothesis (see Chapter 4) states that the pace, processing, quality and quantity of information have increased with digital systems. As such this hypothesis says as much about the physical domain as it says about the digital domain. We will approach properties and differences strictly separate.

\subsubsection{Discovering Properties}

Having defined what properties are, a systematic method is necessary to find the relevant domain properties that play a role in realizing the security goals. As we assume that domain properties affect security through the entities, we therefore investigate the role of each of each entity, how properties

1. determine its possible states;

2. help to realize operations on the entity;

3. contribute to realizing security goals;

4. help to resolve related conflicts between security goals;

5. play a part in the occurrence of threats;

6. contribute to the mitigation of threats.

This is illustrated in Figure 5.8, which shows a schematic overview of a simple KAOS model, which has one instance of each object type. The connectors between the objects (lines, triangles) are specific for connecting different types of objects. However, they do not convey any special meaning and they will not be discussed further here.

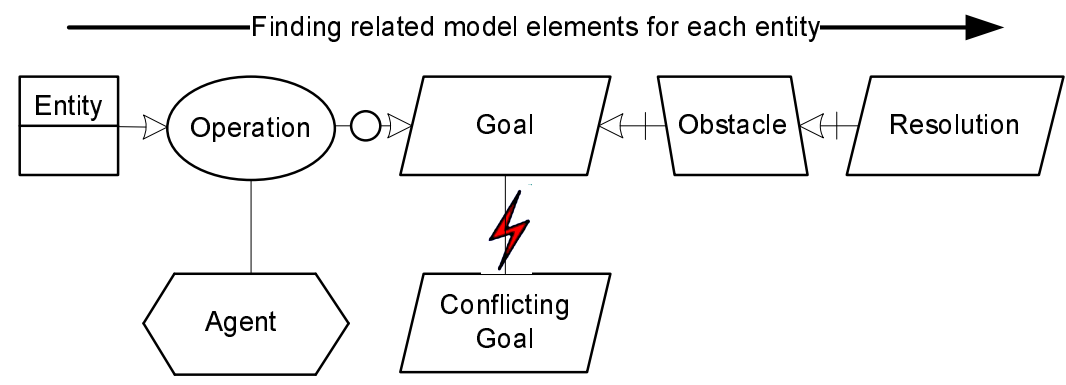

Figure 5.8: Finding related model elements for an entity. 


\subsubsection{Creating and Updating Hypotheses}

After having created a method how to find domain properties, it is necessary to explain the definition of the hypotheses that can be built from these, and how they can be updated.

To begin, each hypothesis consists of a property of a physical or digital entity, which has a specific impact on security under certain conditions. Security is here defined as confidentiality, integrity and availability (see Section 2.3 of information and each impact can be either negative or positive. The hypotheses are therefore of the following form:

$H x$ : physical security property has \{positive|negative $\}$ impact on security $\{$ confidentiality, integrity, availability $\}$ under conditions $\{C\}$

To ensure that each hypothesis is consistent with all case study data, they must be changed when new contradictory data becomes available. In our case, this occurs in two steps:

1. The hypothesis' conditions are changed, such that certain situations are excluded.

2. A new hypothesis is formulated that is consistent with all cases analyzed so far, to be used for the next cases.

Example Consider that there is evidence that visibility allows to detect theft, so that it contributes to confidentiality. This leads to the following hypothesis, unbounded by any conditions:

H1 : Visibility has a positive impact on confidentiality.

However, in another case study a counter example is found: in an election someone can observe the voter when he puts the mark on the paper, which is a threat to confidentiality. Thus the conditions of $\mathrm{H} 1$ must change, such that it only holds for entities that do not directly display confidential information, leading to hypothesis $\mathrm{H} 1$ '.

$H 1^{\prime}$ : Visibility has a positive impact on confidentiality under the condition that entities do not directly display confidential information.

In addition to that, we have a new hypothesis:

$H 2$ : Visibility has a negative impact on confidentiality for objects that display confidential information openly. 
In each of the case studies we document this process with two types of tables:

1. First we explain the newly found properties, their effects on security, as well as counterexamples and conditions.

2. Second we examine the support for hypothesis found in previous cases, again including counterexamples and conditions.

\subsubsection{Relation between Positive and Negative Effects}

As mentioned, a property can improve or reduce security. In both cases, a counterexample to the hypothesis can spawn a new hypothesis about an opposite effect. A counterexample to the hypothesis that a property improves security, can lead to a second hypothesis that the property also reduces some aspect of security. This dual nature of effects is not absolute: not all counterexamples lead to a new hypotheses, and not all hypotheses have opposite relations:

- Whether a counterexample leads to the creation of a new hypothesis is dependent on the domain: as mentioned we will not investigate the social domain in detail and focus on the physical and digital domain. Only if the conditions remain into the domain of physical security can it lead to a new hypothesis.

- It can also be that a hypothesis simply does not hold under certain conditions, but that these conditions do not lead to the opposite effect - in that case no new hypothesis is added, but instead the current hypothesis is refined by adding conditions to it.

\subsubsection{Inverse Properties}

Some properties are the inverse of other properties, for example visibility and invisibility. The relation is indicated in the cluster: thus both properties "visible" and "invisible" belong to "visual" cluster.

\subsubsection{Data Structure}

Each property is represented in two parts:

- A general part, which lists the general characteristics of the property:

- Property name: the name of the property

- Cluster: a collection of hypotheses concerning one concept (such as visibility)

- Effect: the impact on confidentiality, integrity and availability 
- Hypothesis: explanation why the property produces the effect

- Conditions: the conditions under which the hypothesis is claimed to hold

- Case first observed: the case study in which the property was first observed

- A case study part, which lists relevant observations from the case studies. In the case study part, we distinguish between three types of observations:

- The first (initial) observation of the effect in a case study.

- Observations supporting the initial hypothesis.

- Counterexamples that contradict the original hypothesis.

In turn, each observation has the following form:

- Example: an observation of the effect in the case study.

- Effect: the impact of the observed property on confidentiality, integrity and availability in this example.

- Conditions: conditions under which the hypothesis is true. In the case the observation is a counterexample, this concerns the conditions that exclude the counterexample, under which the hypothesis is true and the factors that contribute to the effect.

Ideally, the final case studies would not introduce any new conditions or contributions, indicating theoretical saturation.

\subsection{Comparison}

For each case, physical and digital systems are compared regarding how they realize security goals. Such a comparison is challenging and it is not possible to give a final verdict on which approach to security is best, physical or digital; the effectiveness of each approach is context-dependent and we try to identify the context-sensitive arguments that can be used to make this trade-off. Physical and digital systems can have strengths and weaknesses that are difficult to compare. We cannot simply count threats and mitigations, nor can we do a risk assessment (based on likelihoods and impacts) because in our cases, and in many real-world case similar to them, there is a lack of information to base such a judgment on. For the comparison, the assumption is made that both digital and physical systems are adequately designed and managed; no meaningful results can come from comparing a badly designed digital system with a sophisticated physical system, or a sophisticated digital system with a badly designed physical system. 
Our reasoning is therefore primarily analytical and begins by listing the security goals and consider for physical and digital systems the hypotheses (hereafter called mechanisms) that impact each security goal.

The results of this analysis are tables with a structure like that in Figure 5.9

\begin{tabular}{lll}
\hline Goal & Physical System & Digital System \\
\hline Confidentiality & + Physical Mechanism M1 & + Digital Mechanism M3 \\
Integrity & \pm Physical Mechanism M2 & + Digital Mechanism M4 \\
Availability & - Physical Mechanism M3 & + Digital Mechanism M5 \\
\hline
\end{tabular}

Figure 5.9: Example comparison of physical and digital systems. Effects of the mechanisms can be positive $(+)$, negative $(-)$, or both depending on the context $( \pm)$.

\subsection{Combinations}

Concerning the combinations of physical and digital security mechanisms (RQ4), we analyzed the different types of hybrid systems in the case study. These combinations are represented in the form of a taxonomy with possibilities to integrate physical and digital security. One combination does not represent one type of system but rather one aspect of it, and multiple combinations can be combined into one system, for example for different components. Thus, these combinations are in effect design patterns [51] that are used for the application of results by practitioners. The construction of design patterns is explained in Section 5.12 .

\subsection{Trade-off Analysis}

We will now further analyze the concept of trade-off analysis that is used to assess the benefits and limitations of physical, digital and hybrid systems. To begin, in a trade-off analysis options are evaluated based on their desired properties, with the objective to find the option that most adheres to the desired properties. For example for the development of different types of power plants, their construction cost and environmental pollution would be examined with the objective to find a power plant that has both a low cost and little environmental pollution. Such an analysis is often done when desired properties are known to conflict, meaning that achievement of one goal makes the realization of another goal more difficult.

In the case of security goals, we already observed in Section 2.3 that these goals can be realized in mutually conflicting ways and thus conflicts are to be expected. Therefore, the trade-off analysis in this research concerns a situation 


\begin{tabular}{lcc}
\hline Goal & Hybrid Compared to Physical & Hybrid Compared to Digital \\
\hline Confidentiality & ++ & + \\
Integrity & - & $=$ \\
Availability & - & $=/+$ \\
\hline
\end{tabular}

Figure 5.10: Example table structure for the comparison of hybrid with physical and digital systems. (The example is not based on any real data.)

where the desired system properties are security goals, and the possible options are determined by the part of the system that is digital or physical.

The objective is then to find those combinations of physical and digital systems and security mechanisms that best realize security goals. In this situation it is not clear what the problem structure is as both physical and logical security mechanisms have strengths and weaknesses. For example it is possible that combinations of physical and digital security amplify each other's weaknesses, but it is also possible that they compensate for each other. A priori we have three hypotheses about these combinations.

H1 Accumulation of negative properties:

A combination amplifies the worst properties of physical and digital systems. For example the attack surface may be increased if a system has both physical and logical components.

H2 Compensation:

Negative properties are limited by a positive property or vice versa. For example a physical security weakness can be compensated by stronger digital security, or a physical security strength can be reduced because of a digital security weakness.

H3 Accumulation of positive properties:

A combination amplifies the best properties of physical and digital systems. For example an attacker may have to hack both the physical and the digital system to mount a successful attack.

To test these hypotheses we compare hybrid systems to digital and physical systems. For each case this is done using a table of the structure shown in Figure 5.10.

In essence we look at every hybrid system and assess for each goal if it performs better or worse than a physical system (Column 2) or a digital system (Column 3). The assessment is based on the effects of the properties on the hybrid system. For example, a physical system will be affected by properties that have 


\begin{tabular}{|c|c|c|}
\hline Score & Definition & Operationalization \\
\hline++ & $\begin{array}{l}\text { Hybrid is much } \\
\text { better }\end{array}$ & $\begin{array}{l}\text { The physical or digital system cannot adequately } \\
\text { satisfy the goal, whereas hybrid can. }\end{array}$ \\
\hline+ & Hybrid is better & Most arguments are in favor of hybrid. \\
\hline$=/+$ & $\begin{array}{l}\text { Hybrid is equal or } \\
\text { better }\end{array}$ & $\begin{array}{l}\text { Logically, hybrid cannot be worse than physical or } \\
\text { digital, and there are arguments in favor of it. }\end{array}$ \\
\hline$=$ & Hybrid is equivalent & $\begin{array}{l}\text { Arguments of hybrid and physical or digital security } \\
\text { are similar. }\end{array}$ \\
\hline \pm & $\begin{array}{l}\text { Depends on } \\
\text { circumstances }\end{array}$ & $\begin{array}{l}\text { There are strong arguments in favor of hybrid, but } \\
\text { also against hybrid. }\end{array}$ \\
\hline$=/-$ & $\begin{array}{l}\text { Hybrid is equal or } \\
\text { worse }\end{array}$ & $\begin{array}{l}\text { Logically, hybrid cannot be better than physical or } \\
\text { digital, and there are arguments against it. }\end{array}$ \\
\hline- & Hybrid is worse & Most arguments are against hybrid. \\
\hline-- & $\begin{array}{l}\text { Hybrid is much } \\
\text { worse }\end{array}$ & $\begin{array}{l}\text { The physical or digital system can adequately satisfy } \\
\text { the goal, whereas hybrid cannot. }\end{array}$ \\
\hline
\end{tabular}

Figure 5.11: Assessment scores of comparing hybrid with physical and digital systems.

both positive and negative effects. The hybrid system can be subject to the same effects and we assess which of the following possibilities is applicable:

- Positive effects of physical properties are not or to a lesser extent present in the hybrid system as compared to physical systems. In this case the hybrid system is worse.

- Negative effects of physical properties are not or to a lesser extent present in the hybrid system as compared to physical systems. In this case the hybrid system is better.

Next, the results of this comparison are aggregated, and Figure 5.11 shows the values of assessments, how they are defined and operationalized. It must be stressed though that, even though it was attempted to provide clear guidelines, these elements will remain expert judgments based on the data in the case studies, and future research may give reasons to improve these judgments. For each table presented in the case studies, we indicate the section in which the motivations for each assessment can be found.

\subsection{Application of Results}

The results of the case studies are applied in two methods for the assessment of existing systems and for the design of new systems. The analysis method for the first follows the identification of the relevant properties for that system that was 
explained in Section 5.8. The foundation of the design method is the taxonomy of hybrid systems, which are presented as security patterns. In presenting the patterns, we will follow the notation by Gamma et al. [110](Figure 5.12).

\begin{tabular}{|c|c|}
\hline Element & Description \\
\hline Intent & $\begin{array}{l}\text { The problem solved by the design pattern and its general } \\
\text { rationale and purpose. }\end{array}$ \\
\hline Also Known As & Other names for the pattern, if any are known. \\
\hline Example & $\begin{array}{l}\text { A real-world example demonstrating the existence of the } \\
\text { problem and the need for the pattern. }\end{array}$ \\
\hline Motivation & $\begin{array}{l}\text { A description of situations in which the pattern may apply and a } \\
\text { more detailed description of the problem that the pattern is } \\
\text { intended to solve. }\end{array}$ \\
\hline Applicability & $\begin{array}{l}\text { A general description of the characteristics a program must } \\
\text { have for the pattern to be useful in its design or implementation. }\end{array}$ \\
\hline Structure & $\begin{array}{l}\text { A textual or graphical description of the relationship between } \\
\text { the various participants in the pattern. }\end{array}$ \\
\hline Participants & The entities involved in the pattern. \\
\hline Consequences & The benefits the pattern provides and any potential liabilities. \\
\hline Implementation & Guidelines for implementing the pattern. \\
\hline Example Resolved & $\begin{array}{l}\text { An example of how a problem described in the Example section } \\
\text { may be resolved through the use of the pattern. }\end{array}$ \\
\hline Known Uses & Examples of the use of the pattern, taken from existing systems. \\
\hline
\end{tabular}

Figure 5.12: Elements of a design pattern (adapted from Dougherty et al. [51])

The applicability of the two methods (which build on other results of the thesis) is tested in a focus group meeting [138] with security experts. Several security experts from academia and industry were invited to discuss the findings. In the meeting, participants received a presentation about these results, tried to apply these and debated the outcomes in a discussion led by the facilitator.

\subsection{Validity}

Having explained the research methods and designs in the previous sections, the overall validity of the thesis is discussed below. We will begin to explain the different types of validity. In overall, we consider four types 4 .

1. Construct validity concerns justification of the conceptual framework and the constructs that were measured or identified in this thesis, and whether they could be different.

\footnotetext{
${ }^{4}$ C.f. Wieringa, 2004 [227] Part V
} 
2. Conclusion validity concerns whether the conclusions reached are supported by evidence, i.e. can be traced back to the evidence.

3. Internal validity concerns the validity of causal explanations of the observations and whether alternative explanations are possible.

4. External validity concerns the issue whether the results can be generalized to other cases.

Validity is addressed in several locations in the thesis.

1. First in this section, we discuss how the four types of validity apply to the results.

2. Second, each of the four cases has a separate method section. Here the selection of sub-cases (physical, digital and hybrid) is considered, as well as the method of data collection and the scope of the research, and its possible limitations.

3. Third, in the Chapter 10, we look back at all the case studies and again consider the validity of the first five research questions: the properties, the combinations of physical and digital systems (hybrid systems), their comparison and the trade-offs.

4. Fourth, the validity of the results of the focus group meeting is discussed in Chapter 12

We will now discuss each type of validity in more detail.

\subsubsection{Construct Validity}

Throughout the research, we use several basic constructs:

1. security goals

2. physical, digital and hybrid systems

The security goals of confidentiality, integrity, and availability mentioned are explained in Chapter 2. This operationalization is incomplete (as there are other aspects of security) but is widely accepted and has been found to be sufficient for security assessments. Physical, digital and hybrid systems are operationalized in this thesis itself. First, in Chapter 2 the meaning of "physical" and "digital" systems were discussed. A physical system can be understood by understanding its physical behavior, whereas a digital system can be understood by its digital behavior (even though it is running on physical hardware). Hybrid systems were earlier explained as those systems that combine both digital and physical components and must be understood through both their physical and digital behavior. 


\subsubsection{Conclusion Validity}

Regarding conclusion validity, there are four main conclusions that each answer one of the four research questions: (1) about the properties of physical and digital entities, (2) the combinations of physical and digital systems, (3) the comparison between physical and digital systems and the (4) trade-offs between them. Conclusion validity is dependent on the soundness of the research design (the traceability of the data used in the study until the conclusions are reached) and on the execution of that design, namely whether errors were made during the data processing steps (the "coding"). Through the research, data is collected from case studies in a KAOS tree. This is then analyzed. Because KAOS is used, the data is structured in a uniform format, which allows defining a uniform way of processing that data, which is described in Section 5.6. For the properties, combinations and trade-offs this method has been discussed in the Sections 5.8, 5.9, and 5.11. This reduces the chances that the conclusions are not supported by the evidence. The chance of errors in data processing are further reduced by triangulation (using a variety of research methods such as interviews and literature studies) and by carefully verifying the results. For example, after interviews were taken, the stakeholders were asked to comment on the results. A large portion of the actual results was loaded in a special database for consistency checking. This made it also possible to autogenerate many tables, reducing the chances of errors in the final thesis and improving consistency of result reporting in all four main case studies.

Furthermore, much data was collected from the four case studies, and this makes it less likely that individual errors or omissions would have had a great impact on the results. If we for example consider a list of operations for paper voting (Figure 7.3, we find many different threats, of which the omission of one would not severely impact the end result.

\subsubsection{Internal Validity}

Internal validity is the degree of support for a causal explanation of a phenomenon. This translates into three requirements:

1. There is temporal precedence between the "cause" and the "effect".

2. The cause and effect are related.

3. There are no other plausible explanations for the observed relation.

In a randomized controlled trial internal validity can be managed using sampling techniques and a research design with control and treatment groups. However, this is not possible in a case study, because this has a sample of one and we cannot calculate a difference between the difference of getting a treatment or not. Instead 
we can consider the relation between system architecture and security. Thus we investigate the architecture of systems and how components interact to realize security goals. For example we could identify a system architecture that depends on interactions with persons and observe that in such a case it is more difficult for an attacker to launch fully automated attacks, which has a positive effect on security. We will consider internal validity for such arguments in each of the case studies.

\subsubsection{External Validity}

As for our combined case studies and the goal of theoretical sampling, not in all cases did we succeed to apply the case criterion of independence of other case studies. IT infrastructure management depends on access control systems. Furthermore, access control systems and rights management systems are specific variants of what is called "Usage Control" [128]. Finally we have to consider the limited number of cases that we examined. Given time and budget constraints, we researched as many cases as possible, but possibly additional cases could have resulted in different conclusions in the cross-case analysis. The likelihood of this can be assessed with the concept of "theoretical saturation" associated with theoretical sampling. As more cases are added, the amount of new information found in each subsequent case diminishes [53] until saturation occurs. We will return to this issue in Chapter 10, and examine if theoretical saturation has occurred.

Returning to our limited goals for generalizability and our objective of usefulness, our objective was not universal validity, but rather usability of the findings through the aforementioned case-based reasoning (identifying structures in a case that produced phenomena observed in the case, and that are likely to produce similar phenomena in similar cases). Specific generalizable features include the properties of physical entities and the types of hybrid systems as well as their effects on information security. Each of these constructs is different with respect to how it can be validated and this will also be considered in Chapter 10 . In addition to that, once we have analyzed the architecture in the cases and have discovered components with specific capabilities and limitations, we have found generalizable components:

- The components can also occur in other cases, where they can have the same capabilities and limitations.

- The architecture can (with other components) also occur elsewhere, where it is possible to reason in the same way about architecture.

The usefulness of the results is explicitly examined in the focus group meeting, on which we report at the end of the thesis in Chapter 12 . 


\section{Part II}

\section{Case Studies}





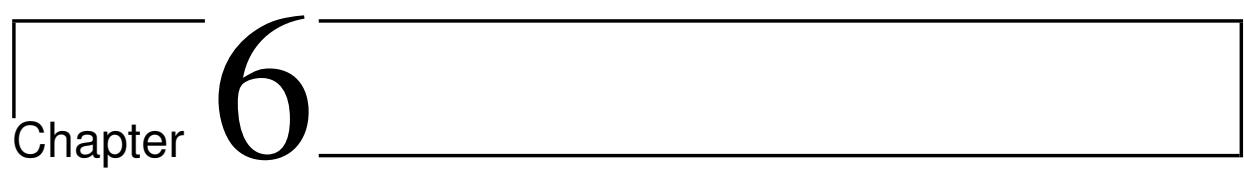

\section{Access Control Systems}

\subsection{Introduction}

In this chapter 1 we will examine several types of access control systems with the purpose of understanding physical and digital security mechanisms. Access control is defined as "the means to ensure that access to assets is authorized and restricted based on business and security requirements" [94]. An asset can be anything of value, even intangible such as company reputation. However, as we are investigating information security, we will assume that the assets to which access must be restricted are information assets. Information assets can be physical (paper) or digital (database records), and access control mechanisms can be realized both in the physical and in the digital domain. As our objective is also to understand combinations of physical and digital security, we will also investigate hybrid access controls. Figure 6.1 shows several examples of how different mechanisms can protect assets.

\begin{tabular}{ll}
\hline Domain & Security Mechanisms \\
\hline Physical & Vault, lock \\
Digital & Encryption, password \\
Hybrid & Digital door lock \\
\hline
\end{tabular}

Figure 6.1: Security mechanisms in different domains.

Physical access control is commonly understood to be the physical protection of physical assets. For example, documents stored in a vault are protected by a lock. Sometimes a physical mechanism can also secure a digital asset, such as

\footnotetext{
${ }^{1}$ This chapter is based on Van Cleeff et al. [209].
} 
when a vault secures an USB stick. Similarly, logical access control typically protects digital assets. For example, digital files are protected by encryption. A logical asset can also secure a physical asset, for example when a passwordprotected web interface controls access to the remotely controlled pumps of a water treatment plant. Hybrid security mechanisms are mixtures of physical and digital protection mechanisms. An example of a hybrid mechanism is a digital door lock that can be opened using a smartphone.

The primary motivation for investigating access control is that it is a core technology for information security: without restricting access and the operations that can be done on information, security cannot be realized. With respect to the standard security properties of confidentiality, integrity and availability, it is clear that especially confidentiality and integrity require restrictions on who accesses data.

A specific focus of this chapter is a form of hybrid access control called "location-based access control". Here a user is granted access based on her location. It is intuitively clear that it should improve logical access control, because a user's location is correlated to the access rights she is entitled to. For example, an HR manager typically has no need for confidential employee data outside of her office and home. Any access to this data from other locations is likely malicious. In addition, location information can help with the authentication of users, because for example a user located in Russia is unlikely to be an employee of an US governmental organization ${ }^{2}$ However, although the benefits of LBAC are intuitively understood, its research and application are hindered by the fact that there is no clear theory that explains how precisely LBAC integrates with and depends on the physical environment [42]. Hulsebosch et al. [88] argue that benefits mainly depend on the value of the resources that LBAC protects, but no general framework exists for deciding which LBAC model is more suitable in which context or for which resource, or in fact, explaining why LBAC actually improves access control at all. We are thus interested in determining its potential, assessing how the integration with physical locations can strengthen logical access control.

The outline of this chapter (and of all subsequent case studies) is straightforward: we begin with an examination of the background and related work, placing the case study into context. Next we discuss our research method, including the selection of sub case studies and data collection methods. We then build up reverse-engineered KAOS models of the systems that were studied, starting with the goals and the systems themselves. We also consider threats to goal realization and potential mitigations. This is done for each type of system: physical, digital and hybrid. After the study of physical systems we answer RQ1 about properties of physical entities. Next we investigate the properties of digital entities (RQ2). Once we understand logical systems we can compare physical and logical

\footnotetext{
${ }^{2}$ Cf. Denning and MacDoran for an early proposal for location-based authentication $47 \mid$.
} 
access controls (RQ3). A subquestion $\left(\mathbf{R Q 3}\right.$ AccessControl $_{\text {) concerns how physical }}$ and logical access control systems make access control decisions. Having reverse engineered a hybrid system, we list possible types of combinations of physical and digital mechanisms (RQ4) and do a trade-off analysis (RQ5). Here we are particularly interested in the benefits of LBAC and its integration with the context

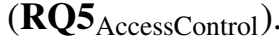

\subsection{Conceptual Framework}

We will now discuss related work concerning access control systems. Although the literature mainly concerns logical access control, the presented concepts are generally applicable, and we will also use them for physical and hybrid forms of access control. After the introduction of several definitions and concepts we explain how we will use these in our research.

\subsubsection{Definition and Concepts}

\section{Reference Monitor}

To begin, the archetypical design for a (logical) access control mechanism is a reference monitor, which mediates between access control requests and the requested objects. Figure 6.2 shows the archetypical reference monitor design. A security administrator manages a database with user authorizations. When users try to access an object, their access requests are sent to the reference monitor. Using the authorization database, the reference monitor makes an access control decision. Optionally, access to objects is logged and can be audited later.

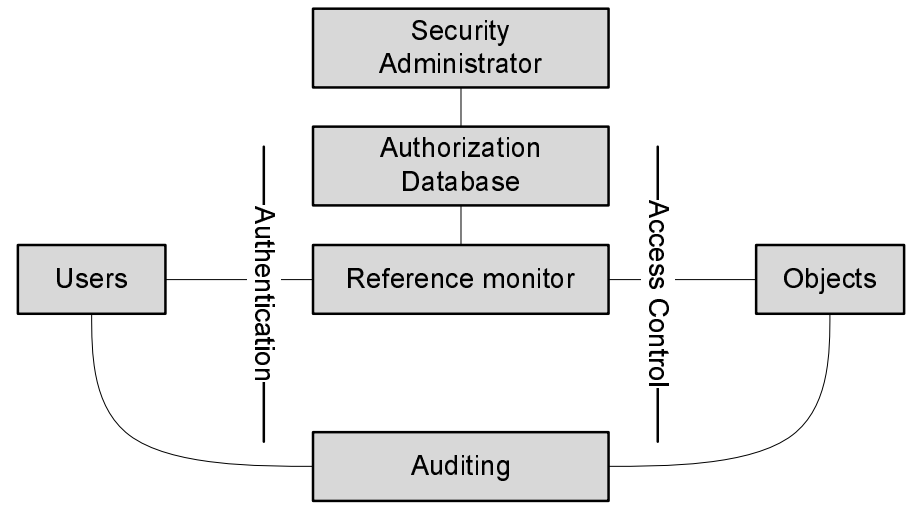

Figure 6.2: A typical reference monitor (adopted from Sandhu and Samarati [185]). 


\section{Usage Control}

Park and Sandhu [161] place access control in the context of usage control (UCON), a broad framework for controlling access to resources. UCON divides access control in two dimensions: payment dissemination and the location of reference monitors (Figure 6.3).

\begin{tabular}{l|l|l}
\hline & $\begin{array}{l}\text { Server-Side Reference } \\
\text { Monitor }\end{array}$ & $\begin{array}{l}\text { Client-Side Reference } \\
\text { Monitor }\end{array}$ \\
\hline $\begin{array}{l}\text { Payment-based } \\
\text { Dissemination }\end{array}$ & DRM \\
\hline $\begin{array}{l}\text { Payment-free } \\
\text { Dissemination }\end{array}$ & $\begin{array}{l}\text { Traditional Access } \\
\text { Control }\end{array}$ & \\
\hline
\end{tabular}

Figure 6.3: UCON dimensions.

Traditional access control models (role-based, mandatory and discretionary) are placed in the quadrant of free dissemination and server-side reference monitors. DRM (see also Chapter 9) is associated with paid dissemination and clientside reference monitors. Access control is split into four hierarchical layers [128]:

1. objectives

2. models

3. architectures

4. mechanisms

First, objectives include general access control objectives related to confidentiality, integrity and availability of information, as well as flexibility and the extent to which these controls are fine grained. Second, models specify how decisions are made. Traditionally, access control determines access to a resource (object) by a particular entity (subject). UCON extends this model with authorizations, obligations and conditions. Third, the technical architecture of the enforcement is based around the concept of a reference monitor, which intercepts access requests of subjects. Fourth, access control must be enforced by security mechanisms, which are discussed by Lazouski et al. [128].

\section{Access Control Services}

Examining access control in more detail, we find that it is comprised of four services or steps [82]:

- identification: uniquely identifying principals 
- authentication: verifying the identity of a principal

- authorization: determining if a principal has access rights

- accountability: auditing access logs to check compliance with security policies

These services depend on each other: without managing identities, one cannot authenticate a principal, and checking authorizations requires knowledge and proof of her identity.

Most access control types in IT systems are logical, and have an authorization function, with inputs Subject, Object and Action and a boolean output: a subject requests permission to perform an action ${ }^{3}$ on an object. Inside the function the decision making takes place.

\section{XACML}

A detailed model for access control is XACML [175]. It is comprised of two parts. The first part is a language implemented in XML that describes the access control policies. The second part is a processing model that describes how to make access control decisions based on these policies. XACML defines several "policy points" that each have a distinct task in access control. Each policy point performs a specific function regarding authorization decisions. In total there are four types of policy points:

- policy information point (PIP): the subsystem storing the information necessary for making an access control decision

- policy decision point (PDP): the subsystem making the actual decision

- policy enforcement point (PEP): the subsystem enforcing the decision

- policy administration point (PAP): the subsystem managing the information stored in the PIP

The functions of these policy points are universal, meaning that any access control system must fulfill these functions. For example we cannot make policy decisions without some form of policy being available, and thus each system must have a form of policy information point to provide this information. Combined, the policy points serve as a reference monitor that intercept actual access requests to perform operations.

\footnotetext{
${ }^{3}$ The term operation instead of action is also used.
} 


\subsubsection{Summary and Implications for Case Study}

Challenges in access control typically focus on authentication, but other functions include identification, authorization and accountability. (In Section 6.3 we consider in how far we include these services.) The reference monitor concept is central to access control as it decides which user is granted access, and we therefore include this concept in the research.

From the UCON framework we learn that there are two main positions for the reference monitor depending on who controls it: either centralized (the system owner) or decentralized (the user). Because this location influences threats and mitigations we specifically examine its location in our research.

A functional decomposition of how access control decisions are made (the model level of UCON) is put forward in the XACML model, which defines policy points with specific functions. As these functions are limited in number (four) and technology neutral, they can be used as operations for the KAOS models constructed in this chapter; using the same basic operations for different systems allows an easy comparison between physical and digital systems.

\subsection{Method}

As outlined in Chapter 5 we will perform case studies on three types of systems: physical, digital and hybrid systems. First, we examine a general synthetic case of completely physical access control, where locks and doors are used. Second, we research a typical case of completely logical access control, where access control is implemented by a software program. Third, we investigate a hybrid case, which is (as already mentioned) "location-based access control" (LBAC). We will now describe our cases in further detail.

\subsubsection{Case Study Selection and Method}

\section{Physical Access Control}

We will begin to investigate a typical physical access control system and determine how it realizes security goals. To achieve a high contrast with logical access control, our system is completely physical, without any logical or electronic components. In our case, we consider an access control mechanism consisting of locks and doors, which protects information assets stored in offices that are located inside a facility. Locks and doors are widely used physical security mechanisms, to be found in every home or office. 


\section{Logical Access Control}

There are several variants of logical access control, including discretionary and mandatory access control. In discretionary access control, a user has the option to change access control rights of objects, such as files in a file system. By contrast, in mandatory access control, access rules are centrally decided and enforced. In our case study, we will use another form called role-based access control (RBAC), as it is one of the most widely researched and applied access control mechanisms.

\section{Hybrid Access Control}

There are many hybrid cases of access control, for example smartcards used to open physical doors. An LBAC system infers the location of a principal through sensors and takes it as input for access control decisions [8]. This allows for the specification and enforcement of location-specific security policies, for example restricting access to sensitive information to a specific room. LBAC is part of the family of context-sensitive access control systems [88], which take all sorts of contextual information as input ${ }^{4}$

Our main reason for selecting LBAC is that it is a relatively recent innovation, of which researchers claim that it has the potential to improve security, but this has never been substantiated in practice. As such, we are interested in finding out what precisely LBAC can add to existing physical and logical access control mechanisms and the emphasis in this chapter is therefore on LBAC.

Literature Study Selecting literature on LBAC was done in three steps. First, we identified relevant keywords to search for, starting with an initial search for "Location-Based Access Control" in titles, abstracts and keywords of papers. Similar terms can be split in two parts: a part on context (such as "spatio-temporal"), and one part on identity and access control (such as "role-based access control"). This is shown in Figure 6.4. In total we created $7 \times 5=35$ keyword combinations.

In the second step, we searched literature using these keywords. We applied the review method of Webster and Watson [224], except for the literature search itself. Rather than examining journal papers and moving backwards to the references, and forward to citations in conferences and workshops, we applied straightforward keyword search criteria, as the scope of our research was well-defined and the set of papers found was relatively small. Concerning the presentation of results, we did follow the approach from Webster and Watson to categorize the results by concept rather than by author, to identify strengths and weaknesses (for example research gaps) in the existing research.

\footnotetext{
${ }^{4}$ There are many terms for these types of systems, which are all put under the "flag" of LBAC, because this term is most widely used and covers our research best.
} 


\begin{tabular}{ll}
\hline Context Term & Access Control Term \\
\hline Context-sensitive & Authentication \\
Context-aware & Authori(s/z)ation \\
Context-dependent & Access control \\
Location-based & Role-based access control \\
Location-aware & RBAC \\
Spatio(-)temporal & \\
Proximity-based & \\
\hline
\end{tabular}

Figure 6.4: Search terms used in the literature study.

In total we found 159 papers with these keywords, which were retrieved in the third step using Google Scholar $1^{5}$. We excluded non-retrievable or irrelevant papers (meaning relevant to our topic), resulting in 99 full papers. In our initial investigation of LBAC, we noticed that most papers did not present any clear motivation for LBAC usage. Instead, they presented LBAC models and examples of their usage. To include literature lacking explicit motivations, we attempted to derive these by following the grounded theory method [35], which allows the development of a theory based on collected data in a structured manner. We collected two types of information:

1. Models of LBAC, how the authors conceptualized relevant events and properties of space-time.

2. Motivating examples on LBAC usage. In total 91 motivational examples were found.

\subsubsection{Limitations and Scope}

Concerning the different access control services outlined in Section 6.2 (identification, authentication, authorization and accountability), we focus on authorizations and pay only limited attention to the identification and authentication of users, assuming that these already have occurred.

Our study of physical access control is confined to a select list of literature. Most of the material used was found in Harris [82] and Anderson [5]. Another source of literature are models of physical protection mechanisms for securing sensitive information inside a facility, as described by Probst et al. [168] and Dimkov et al. [48].

Concerning logical access control, we limited ourselves to investigating standard role-based access control, as it is the most widely used and researched means to assign authorizations to users. (Ferraiolo et al. [57]) We did not thoroughly

\footnotetext{
$\sqrt[5]{\text { http://scholar.google.com }}$
} 
investigate security threats to logical access control nor their mitigations. The reason is that security threats to logical access control are generic and applicable to any IT system: software has vulnerabilities which can be exploited by hackers using specific tools, and errors and flaws in its design and maintenance can also impact security negatively.

As for LBAC, we exclude particular implementations and their specific vulnerabilities from our research. This is not to say that these are trivial. For example when a smartphone serves as a proxy for a person, and is granted permissions, it can still be stolen, no longer signaling the location of the owner. One specific implementation problem for LBAC systems is preserving the privacy of users: determining who has access to the context of the user [88]. Without denying the complexity of these problems, we assume that this problem can be solved satisfactorily. Regarding the literature study, this was limited to scientific literature found on Scopus 6 , including the majority of IEEE, ACM, Springer and Elsevier publications in conferences and journals.

\subsection{Goals}

We will now consider goals for access control. As mentioned in Chapter 2 , information security goals primarily relate to confidentiality, integrity and availability of data. These are in turn realized by security services, such as access control. Access control protects resources against inappropriate or undesired user access. This requires selective sharing of information [87]: neither granting nor denying access to everyone leads to a useful access control system.

As for access control goals, Sandhu et al. specifically mention the principles of least privilege and separation of duty [184], whereas Hu et al. state that there are no well-accepted metrics regarding the goals and desirable features of access control [87]. Instead usefulness depends on the context and the needs of the organization using it. In total, $\mathrm{Hu}$ et al. list 14 specific criteria for evaluating access control systems, including the aforementioned two principles. We therefore include these in our study, as being widely accepted access control goals.

Concerning LBAC, one specific goal on which LBAC is assumed to score well is usability [187]. To test if this is the case, we will include this goal in our study. One goal that has not been addressed in great detail but should deserve further study is maintainability [41], and we also include it. Finally we include the goal of accountability in our study, as it is a known problem of pervasive systems [204] that are a specific application for LBAC.

In total we select five main access control goals in additition to the standard security goals of confidentiality, integrity and availability:

http://www.scopus.com 
1. principle of least privilege

2. principle of separation of duty

3. maintainability

4. accountability

5. usability

Figure 6.5 shows the goals in relation to each other inside a KAOS goal tree.

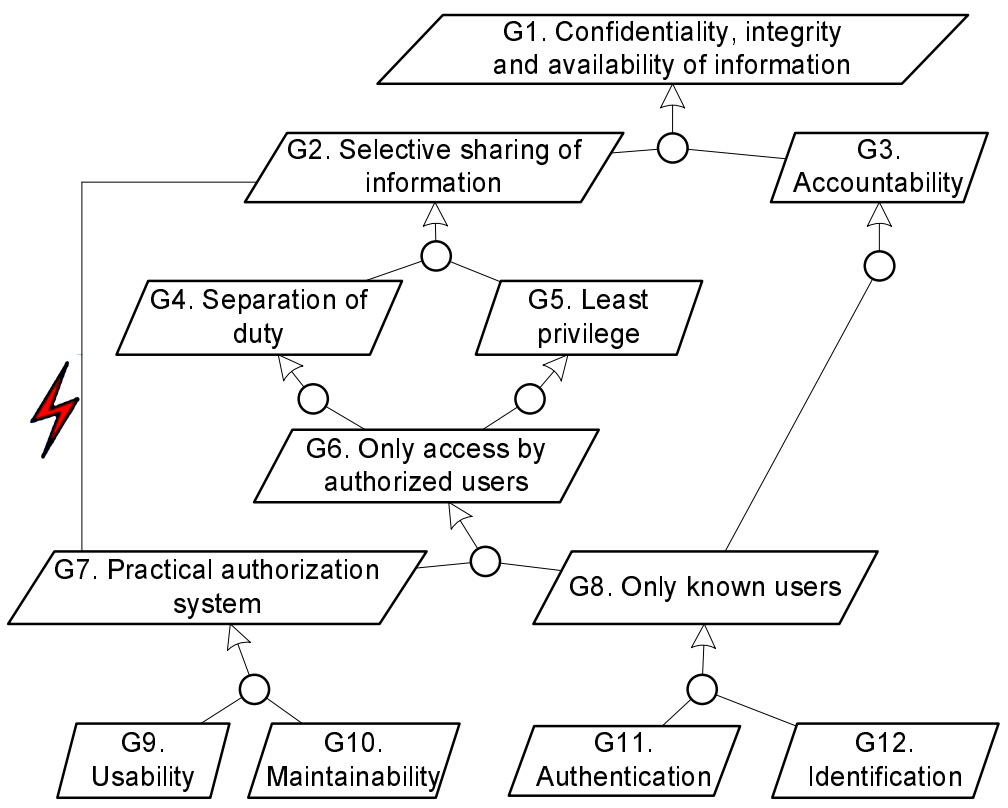

Figure 6.5: Goals of access control.

The top-level goal is to ensure the confidentiality, integrity, availability of information (G1). This is realized by making sure that not everyone has complete access to all information (selective sharing of information, G2), and holding users accountable for their actions (G3). Selective sharing of information means implementing two principles: those of separation of duty (G4) and of least privilege (G5). This requires authorizations to be set (G6), and for these users must be known (G8), meaning they are identified (G12) and authenticated (G11) first. Finally, an authorization systems works only if it is usable (G9) and maintainable (G10). There is one goal conflict, namely between "selective sharing of information" and "practical authorization system" (G7); the more information is shared selectively the more complicated and less practical the system will be and vice versa. We will now discuss the five top-level goals and their goal conflicts, 
because we will use these goals to evaluate physical, digital and hybrid access control systems in the coming sections.

\subsubsection{Principle of Separation of Duty}

Because principals are not always trustworthy, actions must be split between users, allowing each one to verify the other, or depend on the other for execution. Separation of duty (SoD) is possible in time (workflow), or using a two-man or dual control policy, which requires multiple persons to approve an action. Toahchoodee and Ray [203] list two types of SoD: static SoD means that users do not have conflicting roles or permissions, dynamic SoD means that users cannot activate conflicting roles during the same session.

\subsubsection{Principle of Least Privilege}

In theory, access control allows the implementation of the principle of least privilege [182]: access to resources should only be granted when necessary for legitimate purposes. Implementation of this principle limits the risks. First, it prevents actors from making mistakes when they have too many authorizations. Secondly, it prevents malicious actors from abuse, as they cannot access everything. Third, it prevents anyone impersonating the actor (for example because of password theft) from accessing more data than the actor was entitled to access.

\subsubsection{Accountability}

In practice, the principle of least privilege is hard to implement, because in the interconnected dynamic world of today, no one knows precisely what access rights are necessary for legitimate purposes. Instead, users should be held accountable for their actions [27]. This is done by logging and monitoring these actions. It can also act as a deterrence, and in some case even allow recovery from illegal actions.

\subsubsection{Maintainability}

The problem of adherence to the principle of least privilege leads to a fourth goal, namely maintainability. Administrators must keep track of what users are authorized to do, and keep authorizations synchronized to their job descriptions. One of the problems of access control concerns the management: the policy enforcement point can enforce the general policies, but at some point these policies will be subjected to change. (In XACML terms, the policy administration point must take care of this, and this leads to the question of how the access to the PAP itself is managed.) 


\subsubsection{Usability}

Finally, an access control system that puts a heavy burden on its users increases the chance of being circumvented and defeats its own purpose. For example users faced with a system that requires many role changes and passwords will write them down because they cannot remember them.

\subsubsection{Goal Conflicts}

The goal of "selective sharing of information" implies a fine-grained access control mechanism, implemented through the principles of separation of duty and least privilege. However, fine-grained mechanisms have a negative impact on usability and maintainability: the more options and configurations the access control mechanism requires, the more difficult it will be to use and maintain. Likewise, the easier the access control system is to maintain and use, the less fine-grained and selective it will be. We will return to this conflict later in our assessment of physical and digital access control mechanisms, to find out how different access control mechanisms handle this conflict.

\subsection{Physical Access Control}

We will begin the study of access control mechanisms with a physical system and relate its operations and entities to the goals described in Section 6.4 Also, we investigate threats (obstacles in KAOS terms) against physical access control, and consider mitigations (resolutions in KAOS terms) of them.

Figure 6.6 shows an archetypical layout of a facility, similar to other models used in physical security modeling such as by Probst et al. [168]). This facility is equipped with a physical access control system consisting of locks and doors. After entering through several offices, employees can get physical access to the servers.

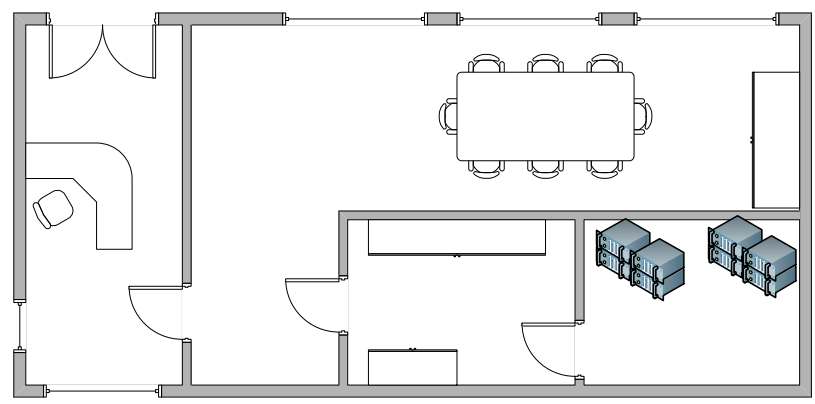

Figure 6.6: Typical layout of a facility. 
The facility consists of basic physical structures such as walls, corridors and doors. The entrance is protected by guards. Employees work in offices, which have doors with locks. There are thus several entities of interest in both the context and the system shown in Figure 6.7.

For this system (as for the other logical and hybrid systems) we list the entities and the operations performed on them. The XACML standard discussed in Section 6.2 helps us to find the operations for access control, as each policy point performs one operation:

- PIP: store policy information

- PDP: determine access rights

- PEP: enforcing policies

- PAP: manage the policies

In an addition to these operations, a user also sends access requests to the access control system itself. This is the fifth operation. Figure 6.8 lists the operations in physical access control.

\begin{tabular}{llll}
\hline ID & Name & ID & Name \\
\hline E1 & Employee & E5 & Key \\
E2 & Manager & E6 & Office \\
E3 & Door & E7 & Hallway \\
E4 & Lock & & \\
\hline
\end{tabular}

Figure 6.7: Entities used in physical access control.

To begin, there is the storage of policy information $(\mathbf{P 1})$ : the lock stores the policy information such that the door can be opened with the appropriate key. The lock determines the access rights (P2): if the key fits the lock, the person holding the key can open it. Furthermore, the lock enforces the policies. (P3). Next is the management (P4), which involves (i) changing the lock, (ii) handing in and handing out keys to employees, and (iii) moving assets to a different location. Finally there is the actual usage (P5): the employee walks to the door and enters the key into the lock.

\subsubsection{Threats and Mitigations}

We will now examine the threats against physical access control mechanisms and how to mitigate these. Figure 6.9 shows the list of threats. Each " $X$ " indicates that the treat impacts a specific goal. We explain the threats further below. 


\begin{tabular}{lllll}
\hline ID & Operation & Goal & Agent & Entities \\
\hline P1 & Store Policy Information (PIP) & G1 & Lock & \\
P2 & Determine Access Rights (PDP) & G2 & Lock & \\
P3 & Enforce Policies (PEP) & G2 & Lock, door & \\
P4 & Manage the Policies (PAP) & G6, G10 & Manager & Key \\
P5 & Send Access Requests & G2, G9 & Employee & Key \\
\hline
\end{tabular}

Figure 6.8: Operations used in physical access control.

\begin{tabular}{|c|c|c|c|c|c|c|}
\hline ID & Threat & & $S^{0}$ & 5 & $\nabla^{\mathcal{c}}$ & \\
\hline $\mathrm{T} 1$ & Key duplication & $\mathrm{X}$ & & $\mathrm{X}$ & $\mathrm{X}$ & \\
\hline $\mathrm{T} 2$ & Key theft or loss & $\mathrm{X}$ & $\mathrm{X}$ & $\mathrm{X}$ & $\mathrm{X}$ & \\
\hline $\mathrm{T} 3$ & Direct attacks on locks & $\mathrm{X}$ & $\mathrm{X}$ & $\mathrm{X}$ & $\mathrm{X}$ & $X$ \\
\hline $\mathrm{T} 4$ & Attacks on persons & $\mathrm{X}$ & $\mathrm{X}$ & & $\mathrm{X}$ & \\
\hline T5 & Tailgating & $\mathrm{X}$ & $\mathrm{X}$ & & $\mathrm{X}$ & \\
\hline
\end{tabular}

Figure 6.9: Threats to physical access control goals.

Key Duplication As the shape of the key expresses the information needed to duplicate it, one attack is to copy the shape (T1). This can be done when the key is under the attacker's control (simply copying it at the locksmith), but also at a distance by making a photograph and extracting the key shape from it. Laxton et al. demonstrate the practical feasibility of this [127]. A wide range of techniques exist to duplicate the key, but 3D printers which can create physical structures make the process very easy [163]. (This is an example of digital attacks against a physical system, we will return to this later.) To prevent duplication, Patel proposes to hide the shape of the key, but conclude that ultimately the security should be based on an invisible secret, such as an RFID chip. This might lead to the conclusion that physical security with standard keys is essentially broken, but in practice these attacks are rare. The practicality of such attacks depends on three factors:

- Can an attacker observe the keys in sufficient detail?

- Does the attacker (or an accomplice) have access to material to generate the keys?

- Can the attacker (or an accomplice) actually use the key? 
If we consider a prison, then prisoners might be able to observe the keys, but not be able to photograph them. Even if they would be able to retain the image, the prisoners still must manufacture the key, or smuggle it inside. Finally, they would also need access to the door itself. If these activities are very costly or impossible to perform in practice, a physical key system can be still be quite secure.

Key Theft or Loss Regardless of whether a key is duplicated, a key can be stolen from the user, or she can lose it (T2) and it can be used by the attacker, because the lock cannot check who holds the keys.

A mitigation is that the rightful owner can detect the loss because it is a physical item. If policies are strict, the user must carry many keys which complicates key management for a person and increases the likelihood of loss. A hierarchical key system (where a key can open many doors up to a certain security level) requires fewer keys and can mitigate this problem.

Many organizations also have a need for master keys, which are then kept at "secret" locations, only known to some, and this system is prone to abuse as keys are no longer personal and the goal of accountability is violated.

Direct Attacks on Locks Locks themselves can also be attacked as they can be forced (T3). Although the lock does not have any access log, the attack might leave detectable, visible traces that can alert persons that a security breach has taken place.

Attacks on Persons Persons in the facility are also vulnerable to attacks. They can either knowingly (for example by bribing) or unknowingly (after social engineering) help the attacker (T4). In our system, an employee can aid the attacker in four ways:

- hand out keys

- open doors, or fail to close them

- ignore attacks

- move sensitive documents

Tailgating A weakness in lock security mechanisms is that a door cannot control what happens after the door is opened, for example how many people enter. This is the threat of tailgating: multiple persons getting through a door while only the first person is allowed to do this (T5). Mitigations include monitoring and turn stiles. 


\begin{tabular}{|c|c|c|}
\hline Property & Effect & Hypothesis \\
\hline Decaying & IA- & A decaying object becomes damaged and unusable. \\
\hline Dense & $\mathrm{CIA}+$ & $\begin{array}{l}\text { A dense physical object acts as a barrier for persons who } \\
\text { cannot get inside. }\end{array}$ \\
\hline $\begin{array}{l}\text { Easily } \\
\text { manufacturable }\end{array}$ & CI- & $\begin{array}{l}\text { If an object is easy to manufacture this reduces its } \\
\text { uniqueness and threatens any process that depends on it. }\end{array}$ \\
\hline Invisible & $\mathrm{C}+$ & $\begin{array}{l}\text { Invisible information cannot be stolen without attacking } \\
\text { the object that contains it. }\end{array}$ \\
\hline Limited spacious & $\mathrm{CIA}+$ & $\begin{array}{l}\text { The small size of a location reduces the opportunities for } \\
\text { an attacker to hide herself. }\end{array}$ \\
\hline Slow & $\mathrm{CIA}+$ & $\begin{array}{l}\text { Slow objects remain close to their intended location, } \\
\text { requiring an attacker to travel. }\end{array}$ \\
\hline Strong & $\mathrm{CIA}+$ & $\begin{array}{l}\text { A strong physical object protects confidential } \\
\text { information. }\end{array}$ \\
\hline Strong & CIA- & $\begin{array}{l}\text { A strong physical object is useful to break a security } \\
\text { mechanism. }\end{array}$ \\
\hline Visible & $\mathrm{C}-$ & An attacker obtains information when it is visible to her. \\
\hline Visible & $\mathrm{CIA}+$ & $\begin{array}{l}\text { An object's visibility allows a defender to deter, detect } \\
\text { and respond to threats. }\end{array}$ \\
\hline Visible & CIA- & $\begin{array}{l}\text { An attacker observes a system and uses this information } \\
\text { to attack it. }\end{array}$ \\
\hline Weak & $\mathrm{CIA}+$ & $\begin{array}{l}\text { An attacker cannot bypass security mechanisms because } \\
\text { she lacks physical strength. }\end{array}$ \\
\hline
\end{tabular}

Figure 6.10: New hypotheses from the access control case study about the security effects of physical properties of entities. Each row represents either positive or negative effects. Multiple letters indicate an effect on multiple security goals. For example CIA- means a negative effect on confidentiality, integrity and availability.

\subsection{Properties of Physical Entities}

Having investigated how physical access control can realize goals, we are ready to assess how properties of physical entities help realize these goals (RQ1). This is done through the method discussed in Chapter 5. First, Figure 6.10 shows the list of newly discovered properties and their impacts. In the next paragraphs, each property is described in more detail.

Decaying Wear damages physical items, changes them and makes them unusable. An example specific to this case is that wear damages keys, changes them (integrity) and makes them unusable (availability).

Dense A physical object acts as a barrier for persons to get in and attack a system. An example specific to this case is that a wall protects documents inside a 
facility. A person cannot walk through walls and therefore walls improve security. A counterexample is that a person already has access through a key. This leads to one condition:

- The attacker has no alternative means to obtain access.

Easily Manufacturable Manufacturing an object threatens the confidential information of the object itself and the confidential information to which it gives access. Therefore objects that are easy to be manufactured have a negative impact on the confidentiality and integrity of a system. An example specific to this case is that copying a capability such as a key threatens the confidential information to which that capability gives access. Also an attacker that copies a key threatens the confidentiality of documents inside a location. A counterexample is that an attacker lacks the ability to copy the key. The object is not a capability to access something. This leads to two conditions:

- The attacker has the capability to reproduce the object.

- The objects act as a capability to access something.

Invisible Invisibility has a positive effect on confidentiality because information that is not visible cannot be stolen without attacking the physical item that contains it. An example specific to this case is that a lock hides the key structure that it accepts. A counterexample is that the object does not hold confidential information. This leads to two conditions:

- No confidential information is stored.

- There is a physical object that contains confidential information.

Limited Spacious Locations that are small in size offer limited opportunities to attackers to hide themselves or their equipment because they are easily detected. An example specific to this case is that attackers and equipment can be found easily in a small room. A counterexample is that the facility is large and cannot be overseen. This leads to one condition:

- The location in which a process takes place is small in size.

Slow Physical objects cannot move very fast, and are thus likely to remain close to their intended location (availability and confidentiality) and also remain unchanged (integrity). Thus it requires effort by an attacker to travel to the facility where the attack takes place and bring the necessary tools. An example specific to this case is that an attacker must travel to the facility. A counterexample is that the attacker is already present, for example as an insider. This leads to two conditions: 
- There are no insiders.

- The attacker is external and located somewhere else.

Strong The strength of an object protects its integrity, availability and the confidentiality of its contents. Similarly, but slightly different is the situation when a physical object does not hold confidential information itself, but merely acts as a barrier to the information. For example, a door protects the security of documents inside a location. A contributing factor is when this door is actually monitored by a guard. An example specific to this case is that a door protects the security of documents inside a facility. The strength of the lock protects its integrity and availability and the confidentiality of its confidential internal shape. A counterexample is that the object does not hold confidential information. Also an attacker can break the lock. This leads to two conditions:

- A physical object contains confidential information.

- The physical protection is strong enough to withstand attack.

Strength can also negatively impact security when a strong physical object is used to break a security mechanism. An example specific to this case is that a physical object is used to attack a security mechanism. A lock picker has tools to open locks.

Visible A negative aspect of visibility is that an attacker can observe a business process (or a specific security process to protect it) and use this information to attack it. For example, an attacker observes in which locations confidential data is stored and how it is secured, and uses that information to attack when there is the least chance of detection. An example specific to this case is that an attacker obtains confidential information when it is visible to her, for example a physical key. Also an attacker takes a picture of the physical key and duplicates it. A counterexample is that an attacker cannot remember or photograph a key. This leads to two conditions:

- There is a physical object that displays confidential information.

- The attacker should have the means to copy or remember the data: for example a physical key must be copied or photographed with a camera.

The visibility of an object allows a defender to deter, detect and respond to security threats because these threats manifest themselves visibly, which has a positive aspect on all aspects of security. An example specific to this case is that an employee notices the theft of key. A counterexample is that the visibility of the key also has a negative effect on confidentiality. This leads to one condition: 
- The physical object does not expose confidential information itself.

Another negative aspect of visibility is that an attacker can observe a business process (or a specific security process to protect it) and use this information to attack it, which has a negative effect on all aspects of security. An example specific to this case is that an attacker can observe how a process is secured and use that information to attack when there is less chance of detection. A counterexample is that an attacker is not capable of observing the process (for example she is outside of the facility). The attacker has no capabilities to exploit her knowledge because example she cannot duplicate a key. This leads to two conditions:

- An attacker observes the process.

- An attacker exploits weaknesses in the security mechanisms.

Weak In contrast to the positive effects of strength, weakness can also have a positive effect on security. This is the case when persons cannot easily bypass security mechanisms such as doors, locks and walls because they lack the physical strength. An example specific to this case is that an attacker cannot easily break down a wall.

\subsection{Logical Access Control}

We will now move on from physical system to logical systems and research role-based access control. RBAC assumes that access must be constrained for certain operations on objects by users [57]. To prevent defining the association between users and operations for each user individually, users are placed into roles that are in turn associated with operations (Figure 6.11)

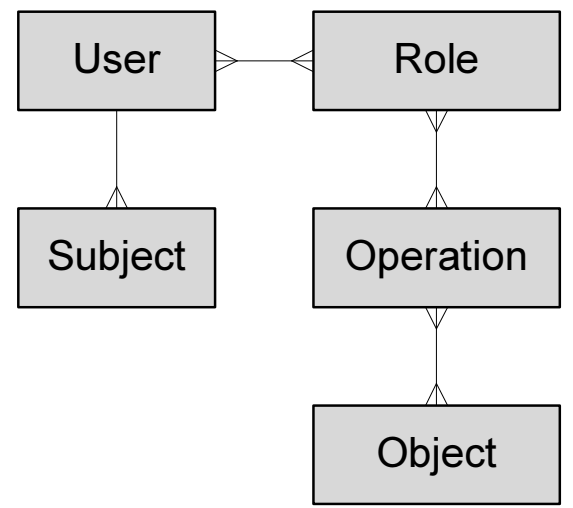

Figure 6.11: The RBAC model. 
In this way, a security administrator only has to associate roles with operations once, after which she can simply place users into roles. For further ease of use, roles can form hierarchies, where roles contain each other: for example in a laboratory, anyone in the role of "lab analyst" can enter lab results, and the role "head of the laboratory" contains the role of analyst, such that she can also enter results, with an additional operation of signing off lab reports. In RBAC, a subject represents an active user process, and each subject has a number of authorized roles at a given time.

As is clear from Figure 6.2, there are several entities involved in logical access control. For reference these are listed again in Figure 6.12. Figure 6.13 shows the operations for logical access control.

\begin{aligned} & \hline ID Name \\ & \hline E01 Reference monitor \\ & E02 Authorization database \\ & E03 Objects to be secured \\ & \hline\end{aligned}

Figure 6.12: Entities used for logical access control.

To begin, a reference monitor stores the policy information in a datastore $\mathbf{P 1}$. The decision making process (P2) for whether a user can perform an operation is straightforward: (i) Find the roles that a subject is in, (ii) find the corresponding set of operations, and (iii) check if the operation is contained within this set. Policy enforcement (P3) is done by the reference monitor and an administrator manages the policies (P4). It consists of three activities: (i) changing the authorization policies, (ii) changing the authentication policies, and (iii) relabeling the assets. Finally users send access requests to the reference monitor (P5).

As explained in Section 6.3.2, we do not consider threats to logical access control. Thus, we directly continue with the next section, where we investigate the digital security properties of logical access control (RQ2).

\subsection{Properties of Digital Entities}

Figure 6.14 shows the digital property that was identified in the access control case.

Accurate Data Storage, Transportation And Processing Given the fact that no threats were investigated in this case, there is only a positive contribution, namely that accurate handling of data has a positive effect on information security. An example specific to this case study is that accuracy helps to define access control policies in detail, and implement them correctly without errors. A 


\begin{tabular}{|c|c|c|c|c|}
\hline ID & Operation & Goal & Agent & Entities \\
\hline P1 & Store Policy Information (PIP) & G1 & $\begin{array}{l}\text { Reference } \\
\text { monitor }\end{array}$ & $\begin{array}{l}\text { Authorization } \\
\text { database }\end{array}$ \\
\hline $\mathrm{P} 2$ & Determine Access Rights (PDP) & G2 & $\begin{array}{l}\text { Reference } \\
\text { monitor }\end{array}$ & $\begin{array}{l}\text { Authorization } \\
\text { database }\end{array}$ \\
\hline P3 & Enforce Policies (PEP) & $\mathrm{G} 2$ & $\begin{array}{l}\text { Reference } \\
\text { monitor }\end{array}$ & $\begin{array}{l}\text { Authorization } \\
\text { database }\end{array}$ \\
\hline $\mathrm{P} 4$ & Manage the Policies (PAP) & G6, G10 & Manager & $\begin{array}{l}\text { Authorization } \\
\text { database }\end{array}$ \\
\hline P5 & Send Access Requests & G2, G9 & Employee & $\begin{array}{l}\text { Authorization } \\
\text { database }\end{array}$ \\
\hline
\end{tabular}

Figure 6.13: Operations used in logical access control.

\begin{tabular}{lll}
\hline Property & Effect & Hypothesis \\
\hline $\begin{array}{l}\text { Accurate Data Storage, } \\
\text { Transportation and Processing }\end{array}$ & CIA+ & Accuracy of IT increases security. \\
\hline
\end{tabular}

Figure 6.14: New hypotheses from the access control case study about the security effects of digital properties of entities. Each row represents either positive or negative effects. Multiple letters indicate an effect on multiple security goals. For example CIA- means a negative effect on confidentiality, integrity and availability.

counterexample is that the system that is compromised is vulnerable because the property is used against the system by the attacker, having a negative impact on security. This leads to one condition:

- The system is not compromisable (controlled by the organization setting the policies that are accurate and complete).

\subsection{Comparison between Physical and Logical Access Con- trol}

This section compares physical and logical access control. The analysis consists of two parts:

- A comparison of the impact of physical and digital properties regarding confidentiality, integrity and availability, as is done for each case study.

- A comparison between physical and digital access control concerning their ability to realize the specific access control goals from Section 6.4 


\begin{tabular}{llll}
\hline Security Goal & Effect & Physical Property & Digital Property \\
\hline All & + & Dense, Limited spacious, & Accurate Data Storage, \\
& & Slow, Strong, Visible, Weak & Transportation and \\
& & & Processing \\
& - & Strong, Visible & N/A \\
Confidentiality & + & Invisible & N/A \\
& - & Easily manufacturable, & N/A \\
& & Visible & N/A \\
Integrity & - & Decaying, Easily & \\
Availability & - & manufacturable & N/A \\
\hline
\end{tabular}

Figure 6.15: Comparison of physical and digital properties on their ability to realize security goals for the access control case study. This table is constructed from data presented in Figures 6.10 and 6.14

\subsubsection{Comparison of Properties}

Figure 6.15 combines the results from Sections 6.6 and 6.8 and compares physical and digital properties on the realization of security goals.

In this case study, many properties were identified for the physical system but only one for the digital system. (This can be partially due to the fact that we did not investigate threats in this case study, and will change in the next case study in Chapter 7). For physical systems, specific properties can have positive and negative effects. We will now continue with a comparison on specific goals and on decision making.

\subsubsection{Comparison for Least Privilege}

Compared to physical access control, digital access control can be much more fine grained, as it is much easier to create new roles than it is to create new physical structures or physically move assets.

For logical access control, the principle of least privilege is realized by only granting those roles that are necessary to users. As users cannot change roles themselves, this is in principle easily enforced by an administrator. The fact that they are fine-grained has a positive effect on the enforcement of least privilege in digital systems.

Concerning coarse grained physical security, one important contextual factor is the classification of information. If the information is classified hierarchically (from public to secret to top-secret), both the facility and the key system can match this hierarchical classification. The user must move from the outside (public) to the inside of the facility (top-secret) to access more sensitive information, 
demonstrating access at each locked door. Key management is eased if the keys have the same hierarchy, and can open doors up to a certain classification level. For example with classifications top-secret, secret and confidential there are only three types of keys:

- A top-secret key can open doors for top-secret, secret and confidential assets.

- A secret key can open doors for secret and confidential assets.

- A confidential key can open doors for confidential only assets.

In such a case, a coarse-grained access control system is a natural fit for the information and has a positive effect on least privilege.

If the information is not classified hierarchically, least privilege can only be realized by limiting the amount of information stored in each office, such that no person can obtain more then she is entitled to. The administrator then must enforce the policy such that each user only has the necessary key. This complexity and the difficulty to create separate physical spaces have the consequence that security is harder to ensure with a coarse-grained access control system. Combined, the property that a system is coarse grained has a mixed impact on least privilege in a physical system.

\subsubsection{Comparison for Separation of Duty}

The same effects of coarse grained physical security and fine grained digital security found for least privilege are relevant for separation of duty. For physical access control, separation of duty is realized by the physical architecture of the facility that should be split in several offices, which each store different information. Next each individual must be granted access (be given a key) only to the offices that she is allowed to. If a person should be allowed access to location $A$ and denied access to location $B$, this can be realized by only giving that person a key to $A$. There is no automatic mechanism to detect violations of separation of duty. If the person would get a key to $B$ this would not invalidate her access to location $A$. This coarse-graininess has thus a negative effect on separation of duty in a physical system.

For logical access control, separation of duty is easily achieved by granting each person different roles that have different privileges. As role creation is easy, this can lead to very fine-grained access control mechanisms which make it easier to enforce separation of duty. Standard RBAC has no specific construction for conflicting roles (only allow access to $A$ if not access to $B$ ). However, the roles are stored centrally and the administrator can audit the allocated roles by checking the RBAC authorization database, which as a positive effect on separation of duty. 


\begin{tabular}{lll}
\hline Policy Change Level & Physical Access Control & Digital Access Control \\
\hline Roles & $\begin{array}{l}\text { Change locks } \\
\text { change physical layout }\end{array}$ & Change role capabilities \\
Persons & $\begin{array}{l}\text { Change key distribution } \\
\text { Assets }\end{array}$ & $\begin{array}{l}\text { Change user role assignment } \\
\text { Move assets }\end{array}$ \\
\hline
\end{tabular}

Figure 6.16: Comparison of maintainability for physical and digital access control: Steps that are needed to change policies in physical and logical access control.

\subsubsection{Comparison for Accountability}

In a purely physical system, accountability concerns knowing which person has which keys and when they were used. Complete accountability is hard to achieve in a physical system, as the doors (which are the policy decision points) do not register their decisions. Information necessary for deciding access is stored decentrally (in keys and doors) and this makes it difficult to enforce access control because there is no overview of the rules that are in place. Accountability can be improved by strict procedures, for example by handing out keys only for a short period of time, and logging who has access to them. The fact that access is hard to log has a negative impact on accountability. A positive impact on accountability is that users are visible when they access a resource.

In a logical system, accountability is realizable by implementing four policies:

1. Each user should have a unique account such that she is discernible from other users.

2. Users should not share accounts (for example by giving passwords to each other).

3. Users' access should be logged.

4. An administrator should audit the logs regularly.

In contrast to physical security, information about these policies can be stored centrally, and is easier to check, leading to a positive effect on accountability.

\subsubsection{Comparison for Maintainability}

Figure 6.16 directly compares the steps that are needed to change policies in physical and logical access control. For physical access control, maintainability first concerns knowing which keys belong to which users, and what information is stored where. This information is not automatically recorded, and this limits 
maintainability. Second, maintainability is limited because keys are invariably lost: then the keys or the locks must be replaced, or more drastically - the office must be emptied. If only the keys themselves are replaced, the enforcement of access control policies such as separation of duty and least privilege gradually decreases: keys can be momentarily lost and then found again, which might not be logged by the administrator, leading to illegal accumulations of privilege. Furthermore, one option that is not easily available to the administrator is changing the building layout.

For logical access control, maintainability of an RBAC system is helped by the fact that permissions are connected to roles and thus do not have to be associated to each individual separately, and are centrally stored for easy management. However, in large organizations, there are often as many roles as functions, and thus roles do not add as much benefit as can be expected. As this complexity increases the real difficulty in large complex systems is understanding what assets persons actually need access to (implementing the principle of least privilege). As systems become complex users can also accumulate more privileges over time.

\subsubsection{Comparison for Usability}

For physical access control, usability of a physical key system is problematic, especially because of the difficulties associated with transporting keys. First, if users manage their own keys they must remember to bring them when they require access. If a manager hands out keys on a temporary basis, users must return these keys, which is also a burden. Second, strict access control policies can require persons to carry many different keys, further increasing the problems. A hierarchical key system (discussed in the section above on least privilege) can significantly improve usability for keys: in the best case users need only one key.

For logical access control, if each system has a separate user database, the users must remember different user accounts and passwords, which is burdensome. More positive is that systems can help a user switch automatically between roles, such that her only task is to authenticate herself.

\subsubsection{Comparison for Decision Making}

We will answer our research question $\mathbf{R Q 3} \mathbf{3}_{\text {AccessControl }}$ (How do physical and logical access control systems make access control decisions?). This is done by examining the location of the reference monitors and the XACML policy points. If we constrain the analysis to locks and doors, we find that PIP, PDP and PEP are all located inside the lock. The PAP has limited control: a facility manager changes the policies, either by replacing the lock itself or by changing the distribution of the keys. However the employees inside the facility also have a weak role in policy administration and enforcement, as they can detect or violate policies 
with their own keys by observing the people inside. In contrast, for logical access control, the location of the policy points is free - they can be either centralized in one system or distributed. However, the setting is such that there is only one type of policy point per system: there is only one PDP, whereas in physical access control there is one in each lock. As such, physical access control tends to be more decentralized than logical access control.

\subsection{Location-Based Access Control}

We will now examine the benefits of location based access control, a hybrid form of access control that was introduced in Section 6.1. For this we construct a similar KAOS model as for physical and logical access control. However we do not create the figures with lists of operations and entities: these are very similar to those in logical access control. To explain the workings of LBAC, we examine the operations below.

Store Policy Information LBAC systems are a form of context-sensitive access control and thus must store information about the context. Context is defined as "any information that is useful for characterizing the state or the activity of an entity or the world in which this entity operates" [204]. Basic context information is first read from sensors and these inputs can be used to infer high-level context [19]. For example, social behavior of subjects (such as a doctor accompanying a patient), can be inferred from observing the locations of individuals [153].

Low-level context consists of Cartesian $(\mathrm{x}, \mathrm{y}),(\mathrm{x}, \mathrm{y}, \mathrm{z})$ or GPS coordinates. These coordinates are then translated to logical positions (addresses and facilities) by LBAC systems such as GEO-RBAC [40] or STRBAC [125]. A specific logical location type is a country, which has specific legislation [198]. Next to locations, objects are also defined, either physical or logical [173]. On top of logical locations, Zhang et al. [231] also model the hierarchical containment relations between locations, as well as their proximity.

Higher-level context includes history, trajectory of entities, and their closeness, legal status and social behavior. Several authors model position, movement and/or interaction between entities [8], [40], [102]. Ardagna et al. use five predicates to define the context status: disjoint, distance, velocity, density, location density [8].

Determine Access Rights For most LBAC systems, the decision making model is a form of RBAC [43], [115], [140], [171], [173]. Apart from having a certain role, a user then also needs to be in a certain location to perform an action, or activating roles is only possible in a certain location. Authorizations can be revoked if users leave a location. As entities move, access decisions also depend on time, 
it does not only matter where an entity is but also when, for example restricting employees to access data only on the premises of a facility and during working hours. These models can also be extended with specific time restrictions, leading to spatial and temporal access control [18], [28], [125], [172]. Spatio-Temporal RBAC is formalized by Toahchoodee and Ray [203].

An alternative for RBAC schemes is to use mandatory access control (MAC) [170]: objects and locations have certain security levels. Here, moving a highly sensitive device (object) into a low level security zone will disable its features. For example, a computer with top secret information will not work in a public place. Ray and $\mathrm{Yu}$ [173] restrict access to location information using a MAC model. Objects must be contained by locations with higher clearance levels. Apart from role-based and mandatory access control decision models, state checking matrices (SCM) [119], predicates [193], and automata [30] are also proposed.

In most models we investigated, only the subject's location is considered because in many cases, the object location is static [198]. However, for example in case of providing passengers in a moving train with Internet access, the object accessed is also moving [88]. Another exception is mentioned by Park et al., who motivate the inclusion of object location in LBAC for safety reasons [162]. LBAC models can thus use different types of locations [43]: in most cases, the subjects are persons and the objects consist of data or systems at a remote location. An example of an LBAC system that directly impacts the user's device is given by Schmidt et al. [188], who propose to disable a camera in a sensitive location.

Obviously, access control rules based on location of subjects and objects can be extremely fine-grained, for example only allowing access to a medical file when a doctor and a patient are in the same consulting room [153].

Enforcing Policies In LBAC, policy enforcement is done centrally based on the particular access control model. This model bases its decisions on the policies in its database, as well as on the information it receives about the location of the users.

Manage the Policies In LBAC, management of policies is done centrally inside the LBAC system by an administrator. The physical structure remains unchanged, management concerns only logical changes about the applicable policies in each location and for each user.

The maintainability aspect of management is mostly ignored in the literature [41]. An exception is GEO-RBAC where maintenance is split between specific spatial domains and subdomains. The resulting problems are similar to those in inter-organizational context, where organizations use (logical) federated identity and access control systems. LBAC does not solve these problems, but adds a layer of complexity, namely managing physical spaces. Hulsebosch et al. [88] 
state that in any context-sensitive access control system, many different parties will control a part of the context.

Sending Access Requests Depending on the particular LBAC model used, the user either sends an explicit request to the access control system, or is implicitly granted access based on her location or context.

Concerning the usability aspects of this, Sastry et al. [187] argue that LBAC is easy, natural, and familiar in the physical world. They give the example of turning on or off lights in a room: this requires being physically present in the room. As such, LBAC can make access control easier and more natural. Because the location is correlated with a user's identity, LBAC can lower the requirements for authentication. This makes LBAC systems easier to use, especially for always-on systems, such as in hospitals: simply by being close to the system, a doctor can authenticate herself without having to type a long password 7

A drawback of LBAC is that it requires persons to move from one location to the other for performing different tasks. During the course of the normal activities (such as meeting a patient) this is acceptable, but it otherwise hinders usability. Damiani et al. [41] discuss the problem of "domain awareness", similar to the problem of accidental access mentioned earlier by Gupta et al.: a traveler might connect to many different systems and must be made aware of this, without being bothered too much ${ }^{8}$ To solve this, Kirkpatrick and Bertino [120] propose to use enabled and activated roles: the first are always possible, and the latter can only be used when the user has visited a specific location or performed a specific action. Furthermore, we consider the problem of transparency: Kirkpatrick and Bertino state that contextual factors include facts that the user cannot (or does not always) know. For example a person might not be aware of the exact security level of a room and what documents she can access there. If that is the case, this certainly reduces usability.

\subsubsection{Threats and Mitigations}

As mentioned in Section 6.3, we exclude software vulnerability threats from the analysis. It is clear that an LBAC software system can have the same vulnerabilities as any other logical system. As for physical threats, changes in physical access control mechanisms can also affect LBAC indirectly, as these serve as an additional security mechanism. For example a person has to be in a particular office to access data, and this is enforced by a physical mechanism (a key) and a logical mechanism (LBAC). We will also not consider these, as they were covered in Section 6.5. Figure 6.17 shows the remaining list of threats to LBAC.

${ }^{7}$ Cf. continuous authentication systems $[195$

${ }^{8} \mathrm{Cf}$. the problems of notifying travelers of changes in roaming fees of mobile phone networks. 


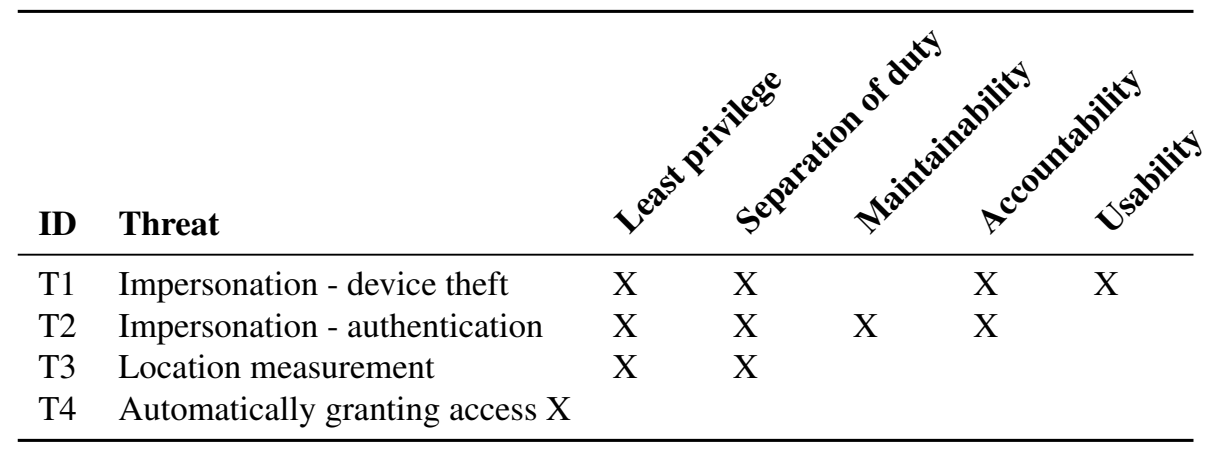

Figure 6.17: Threats to location-based access control goals.

Impersonation Attacks There are two types of impersonation attacks, depending on the particular LBAC system in use. First, an attacker can steal the device that a user carries for location measurement, and thus impersonate the user (T1). Second, if the LBAC systems identify the users with other means such as fingerprints or gait, the attacker could thwart these authentication mechanisms (T2).

Location Measurement Attacks The assumption behind LBAC is that the location of the user can be measured correctly. If this does not happen, the system cannot enforce the policies. Attacks against these measurements can prevent enforcement (T3). For example an LBAC system using GPS is vulnerable to transmitting fake GPS signals [47].

Automatically Granting Access One particular problem with LBAC is automatically granting access to resources. Gupta et al. mention proximity-based access control, and the problem of accidentally granted access [79] (T4): as a user passes by a resource, and is automatically granted access, this violates the principle of least privilege.

\subsubsection{Types of Hybrid Systems}

We are interested in generalizing LBAC system types to other hybrid systems (RQ4). Based on the material presented before, we generalize LBAC as a logical system that takes reliable measurements from the physical environment (or infer information about it) to make security-relevant decisions (Figure 6.18). This holds true for all LBAC systems, regardless of the implementation. 


\begin{tabular}{lll}
\hline$\#$ & Explanation & Example \\
\hline S01 & $\begin{array}{l}\text { Logical system sampling the } \\
\text { physical environment. }\end{array}$ & LBAC \\
\hline
\end{tabular}

Figure 6.18: Types of hybrid systems in the access control case study.

\subsection{Trade-off Analysis}

We will now assess the potential for hybrid mechanisms (RQ5 - What are the trade-offs between physical and digital mechanisms?), starting with the dependencies and main use cases of LBAC (RQ5 $\left.\mathbf{S}_{\text {AccessControl }}\right)$.

\subsubsection{Dependencies and Main Use Cases of LBAC}

Context can be split in legal, IT and social contexts and we discuss these first, before enumerating the main use cases.

\section{Dependencies on Context}

Legal Context Although not widely discussed in the literature, the legal context provides an important motivation for LBAC [198]. Different countries have different regulations in place, and $\mathrm{LBAC}$ can restrict access from specific countries. For example, with a properly secured implementation of LBAC, access from specific countries could be restricted if this would violate company policies or governmental regulations.

IT Context Pervasive or ubiquitous systems, which use sensors, wireless transmissions and/or movable devices, differ from normal systems in their access control requirements. For example if a person walks through a building she should be able to switch on the lights at the place where she is. In such situations, the permission of the person to access objects can strongly depend on the location [86], and this provides a motivation for LBAC.

Hulsebosch et al. argue that in ad-hoc collaborations between users and devices, access control depends more on the context (such as location), and less on identity [88]. Here LBAC can reduce for example the dependency on long passwords. In pervasive systems, identities are either unknown a priori or untrustworthy and cannot be used for standard RBAC [204]: access control should adapt itself to the context.

Social Organization LBAC is aided by the organization of work, especially the specialization of labor: working activities repeatedly take place in the same 
locations and time periods. Jobs can be limited to specific hours [17] and such facts can be used by LBAC mechanisms to implement space and time constraints. The proximity of persons to each other also impacts the need-to-know and needto-do:

- Being alone can improve confidentiality of data, such as in a voting process, where a voter has to fill in a ballot herself, without anyone else being present.

- Being together can make actions more secure because there is more oversight, such as in a "no-lone zone". For example, in an election, the voter must cast the ballot in presence of observers, to make sure that she puts only one ballot in the ballot box?

In other situations, social collaborations are ad hoc, and users do not have a specific identity known to the system or each other, or are unauthorized for a specific task. For example in a conference room, participants can send questions to a panel, which has not authorized the participants individually. An LBAC system that only allows those participants inside the conference room access is useful for three reasons. First, it improves the accountability of persons, who need to be in the room to ask questions and can be identified more easily. Second, it limits the chance of denial-of-service attacks because users must be in the conference room to disrupt the service. Third, it is easy to maintain and use, there is no specific authentication and authorization process necessary as everyone inside the room has automatic access.

Combined, we see four main use cases of LBAC:

1. dynamic or pervasive systems with few security requirements, such as used in conferences or public places

2. static high-security environments with high privacy requirements such as hospitals

3. normal business environments that require a safety net for compliance purposes

4. static high-security environments with physical access control (PAC) such as military facilities

For each of these scenarios, Figure 6.19 shows the typical IT applications, the main goals that can be achieved, the specific LBAC variant that is most applicable and finally the relation to the context.

\footnotetext{
${ }^{9} \mathrm{Cf}$. the concept of natural surveillance 39
} 


\begin{tabular}{|c|c|c|c|c|}
\hline Use case & $\begin{array}{l}\text { 1: Open } \\
\text { systems }\end{array}$ & 2: Hospitals & 3: Enterprises & $\begin{array}{l}\text { 4: } \\
\text { High-security } \\
\text { facilities }\end{array}$ \\
\hline $\begin{array}{l}\text { Typical } \\
\text { application }\end{array}$ & $\begin{array}{l}\text { conference, } \\
\text { museum }\end{array}$ & $\begin{array}{l}\text { electronic } \\
\text { medical files }\end{array}$ & $\begin{array}{l}\text { enterprise } \\
\text { systems }\end{array}$ & $\begin{array}{l}\text { physical } \\
\text { maintenance }\end{array}$ \\
\hline Main goals & usability & least privilege & $\begin{array}{l}\text { accountability, } \\
\text { maintainability, } \\
\text { usability }\end{array}$ & $\begin{array}{l}\text { separation of } \\
\text { duty }\end{array}$ \\
\hline $\begin{array}{l}\text { main LBAC } \\
\text { variant }\end{array}$ & proximity & RBAC & safety net & MAC \\
\hline $\begin{array}{l}\text { context } \\
\text { representation }\end{array}$ & $\begin{array}{l}\text { distance, } \\
\text { proximity }\end{array}$ & $\begin{array}{l}\text { rooms, } \\
\text { collaboration }\end{array}$ & $\begin{array}{l}\text { buildings, } \\
\text { countries }\end{array}$ & $\begin{array}{l}\text { facilities, } \\
\text { rooms }\end{array}$ \\
\hline IT context & $\begin{array}{l}\text { pervasive } \\
\text { systems }\end{array}$ & - & - & - \\
\hline $\begin{array}{l}\text { Physical } \\
\text { context }\end{array}$ & $\begin{array}{l}\text { visibility, } \\
\text { capacity, } \\
\text { reachability }\end{array}$ & $\begin{array}{l}\text { containment, } \\
\text { visibility, } \\
\text { reachability }\end{array}$ & $\begin{array}{l}\text { containment, } \\
\text { PAC, reachability }\end{array}$ & $\begin{array}{l}\text { containment, } \\
\text { travel and } \\
\text { speed limits, } \\
\text { PAC, inertness }\end{array}$ \\
\hline Social context & ad hoc & $\begin{array}{l}\text { known } \\
\text { workforce, } \\
\text { close } \\
\text { collaboration }\end{array}$ & known workforce & $\begin{array}{l}\text { known } \\
\text { workforce, } \\
\text { close } \\
\text { collaboration }\end{array}$ \\
\hline Legal context & - & $\begin{array}{l}\text { compliance } \\
\text { with privacy } \\
\text { regulations }\end{array}$ & $\begin{array}{l}\text { compliance with } \\
\text { business rules } \\
\text { and data } \\
\text { protection }\end{array}$ & - \\
\hline
\end{tabular}

Figure 6.19: Overview of the four main use cases for LBAC. 


\begin{tabular}{|c|c|c|}
\hline Hypothesis & LBAC & Explanation \\
\hline Decaying IA- & $=/+$ & LBAC devices do not wear physically. \\
\hline Dense CIA+ & $=$ & Persons remain dense. \\
\hline Easily manufacturable CI- & $=/+$ & $\begin{array}{l}\text { LBAC devices are harder to manufacture than } \\
\text { keys. }\end{array}$ \\
\hline Invisible $\mathrm{C}+$ & $=/+$ & Secrets in LBAC devices are not visible. \\
\hline Limited spacious CIA+ & $=$ & Locations have the same capacity. \\
\hline Slow CIA+ & $=/+$ & LBAC can use slowness as input for policies. \\
\hline Strong CIA+ & $=$ & Walls remain strong, locks remain strong. \\
\hline Strong CIA- & $=/+$ & $\begin{array}{l}\text { Strength might not be sufficient to steal secret } \\
\text { from LBAC device. }\end{array}$ \\
\hline Visible C- & $=/+$ & $\begin{array}{l}\text { LBAC devices do not show confidential } \\
\text { information like keys do. }\end{array}$ \\
\hline Visible CIA+ & $=$ & All entities remain visible. \\
\hline Visible CIA- & + & $\begin{array}{l}\text { Observations have less impact because. LBAC } \\
\text { device is needed to gain access. }\end{array}$ \\
\hline Weak CIA+ & $=$ & Persons have the same strength. \\
\hline
\end{tabular}

Figure 6.20: Trade-off analysis on the impact of properties between hybrid and physical for the access control case study. Positive scores indicate that the hybrid system is more secure than the physical system, negative scores that it is not. N/A means that the effect was not observed in the case study. (For the exact operationalization details, see Figure 5.11 in Chapter 5) The full explanations of the scores for each goal are Section 6.11.

\subsubsection{Trade-off Analysis on Properties}

Figure 6.20 and 6.21 show the trade-off analysis on the effect of physical and digital properties. To keep the table small in size, the properties and effects (that were put in separate columns in earlier tables) are put together under one column "Hypothesis". The figures show that in most cases, LBAC reduces the negative impact of security properties and enhances their positive impact. This supports hypotheses $\mathbf{H 2}$ (Compensation) and $\mathbf{H 3}$ (Accumulation of positive properties) that were formulated in Chapter 5 .

\subsubsection{Trade-off Analysis on Goals}

Figure 6.22 gives an overview of the trade-offs between physical, digital and location-based access control.

Note that apart from the five specific access control goals, the figure also shows the three generic security goals. The reason is that we will use these in other case studies and we need to compare results between them. The relation between the specific and the generic goals these was shown earlier in Figure 6.5. 


\begin{tabular}{lll}
\hline Hypothesis & LBAC & Explanation \\
\hline $\begin{array}{l}\text { Accurate Data Storage, } \\
\text { Transportation and }\end{array}$ & $=$ & LBAC systems remain accurate. \\
Processing CIA+ & & \\
\hline
\end{tabular}

Figure 6.21: Trade-off analysis on the impact of properties between hybrid and digital for the access control case study. Positive scores indicate that the hybrid system is more secure than the digital system, negative scores that it is not. N/A means that the effect was not observed in the case study. (For the exact operationalization details, see Figure 5.11 in Chapter 5.) The full explanations of the scores for each goal are Section 6.11 .

\begin{tabular}{lcc}
\hline Goal & LBAC Compared to Physical & LBAC Compared to Logical \\
\hline Separation of duty & + & + \\
Least privilege & + & + \\
Accountability & + & + \\
Maintainability & \pm & \pm \\
Usability & \pm & \pm \\
\hline Confidentiality & + & + \\
Integrity & + & + \\
Availability & + & + \\
\hline
\end{tabular}

Figure 6.22: Comparison of LBAC with physical and logical access control on goal realization. A ' + ' indicates that most arguments are in favor of hybrid. A " \pm " indicates that there are strong arguments in favor of hybrid, but also against hybrid. (For the exact operationalization details, see Figure 5.11 in Chapter 5.) The explanation of the scores for each goal is given in Section 6.11 
There is a contribution relation between the five specific access control goals and the generic goals of confidentiality, integrity and availability. Because for most specific access control goals LBAC scores better than physical and digital access control, LBAC also scores better for confidentiality, integrity and availability. The results for the five specific access control goals are further explained below.

\section{Separation of Duty}

Previously we observed that physical access control is coarse grained, and logical access control is more fine grained. This does not mean however that LBAC is between these two extremes. An LBAC system can enforce both logical and physical forms of separation of duty. First, it can enforce physical separation between users, so that they cannot collude directly. Secondly, LBAC can also require the opposite: two users need to be in the same room to supervise each other. Thus LBAC systems have the potential to be more secure than either physical or logical access control and it is scored more positive than both.

\section{Least Privilege}

Similar to separation of duty, LBAC has benefits over physical and digital access control, and can increase adherence to the principle of least privilege, as several LBAC models are very fine-grained, allowing a user access only in a specific data in a specific physical room or while being in proximity of specific people.

\section{Accountability}

Concerning accountability, the problem of physical systems is the lack of an infrastructure for logging, which is present in logical systems. LBAC as a form of logical access control allows logging, but can also make use of physical security mechanisms. LBAC can require a user to be in a certain location to use her authorizations, improving accountability. Malicious usage is detectable when someone visits a location she normally does not, and identity theft is deterred because access requires an attacker to physically visit a location herself, risking detection. Thus LBAC can also improve accountability over both purely physical and logical access control.

\section{Maintainability}

Physical maintainability is hindered by the impossibility to redesign physical structures and the inevitable loss of physical keys. For maintainability of logical systems complexity is a factor as each individual must be granted detailed access rights. 
Given the fact that LBAC adds a layer of complexity to logical access control, namely managing physical spaces, there is a priori little reason to believe that LBAC systems are easier to maintain than their logical or physical counterparts. However, similar to RBAC where permissions are given to roles rather than to individual users, LBAC can improve maintainability by granting permissions to locations rather than to individual users. It can also save administrators time when users and devices in dynamic environments can automatically determine authorizations based on their location. For these reasons, LBAC receives a mixed judgment on maintainability.

\section{Usability}

Different arguments can be put forward about the usability of LBAC; it can be considered a natural way of access control that is also present in the physical world (for example with a light switch), and it can lower the authentication requirements for users. However, it can also be seen as cumbersome, as it forces persons to move between different locations, and it is not always intuitive for a user to what system she connects to, when moving around a building (domain awareness).

Thus, the problems of LBAC usability are different compared to purely physical (carrying keys) or logical access control (switching roles and remembering passwords). Concerning physical access control, it is not clear if LBAC is an improvement, but it can be argued that LBAC is more natural and easier than pure logical access control. As a whole, LBAC therefore receives a mixed score on usability, as compared to physical or digital access control.

\subsection{Conclusion}

\subsubsection{Physical and Digital Properties}

From our analysis, we derive several important properties of physical entities which lead to twelve hypotheses about their impact.

- Visible: Persons and keys are visible.

- Invisible: A door lock does not show which keys it accepts.

- Easily manufacturable: Objects such as keys can be copied.

- Strong: Objects can be strong enough to withstand attacks.

- Weak: Persons lack the strength to break security mechanisms such as walls.

- Slow: Persons move relatively slow, and thus physical access control naturally limits the ability of persons to access unauthorized. 
- Limited spacious: Locations have a limited capacity which helps to discover attackers.

- Dense: Persons are bounded by physical restrictions such as walls.

- Decaying: Items such as keys wear down.

One digital property was discovered, namely "Accurate data storage, transportation and processing", which aids overall security.

\subsubsection{Comparison between Physical and Logical Access Control}

Our investigation in Section 6.9 shows that physical access control, as realized by keys and doors, has inherent strengths and weaknesses. A physical security architecture can be layered, such that it cannot be breached in one step. Indeed a user can make a copy of a key, but this takes time, and the user must access the room herself, risking detection. A (physical) thief must be physically present to access assets. It is also evident that an administrator does not have full control over a physical access control system, as all the policy points are spread over a facility. Physical access control using keys is discretionary. The enforcement is distributed over many doors and locks, and the constructions are such that violations are always possible. Once someone has a key (or access to a room) she can access everything inside the room, and give the key to another person. Still, physical access control allows partial realization of the access control goals specified in Section 6.4 .

By comparison, logical access control is very precise, and much simpler to understand. Compared to physical access control, logical access control is less layered: either a person has access or she does not. The reference monitor is a form of mandatory access control and must function perfectly, or else the system's security is completely breached.

Concerning goal realization, logical security has three main advantages. First, management is centralized, and an administrator can uniformly configure access. Second, access by users can be logged and this increases accountability. Third, logical security policies are much more expressive. For example we cannot specify that a standard physical lock can only be opened during office hours, but logical access control can realize such policies easily.

\subsubsection{Types of Combinations}

Concerning the possible types of combinations of physical and digital systems, we can generalize LBAC as a logical system that takes reliable measurements from the physical environment (or infer information about it) to make security-relevant decisions (Figure 6.18). 


\subsubsection{Trade-off Analysis}

LBAC is vulnerable to the same weaknesses as any software-based access control system (Section 6.11). In how far does LBAC, as a hybrid form then improve security? We can answer this question by seeing LBAC simply as logical access control "plus". An LBAC system makes exactly the same decisions as a logical system, with the addition that it also adds requirements for the location of the user. With this additional requirement, security can certainly be improved, as the attacker must be in a specific - possibly hard to achieve or fake - physical state.

Concerning the goals of separation of duty, least privilege and accountability, we observed that LBAC can improve on both physical and logical security mechanisms. It can make use of the best aspects of both systems. The goal of maintainability does not clearly favor one particular form of system.

As for usability, we can see LBAC as an opportunity for changing logical access control itself, for example allowing authentication and authorization requirements to be lowered. For example, there is no specific authorization necessary to access a resource, the user only needs to be in a certain location.

When the impact of physical and digital properties is considered, it becomes clear that LBAC can improve security over purely physical and digital systems.

Challenges Also, challenges for LBAC remain: how to inform users of applicable policies, and how to do the trade-off analysis between usability and security with respect to automatic activation of authorizations. Furthermore, the existing literature assumes that LBAC is applied in an existing site, but it is not clear how a physical and social structure of a facility should be designed with LBAC in mind.

\subsubsection{LBAC in Context}

We identified four relations between LBAC and its context (Section 6.11.1

1. context as represented inside LBAC

2. IT and legal context as a source of requirements for LBAC

3. physical context as a source of dependencies for LBAC

4. social context as a contributing factor to achieving goals of LBAC

We found four usage scenarios for LBAC: open systems, hospitals, enterprises, and finally high-security facilities such as data centers and military installations. 


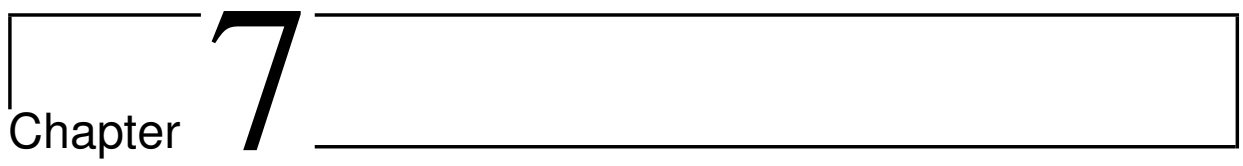

\section{Voting Systems}

\subsection{Introduction}

In this chapter 1 we examine systems that enable citizens to participate in a democratic election. We will call these systems "voting systems". There are many types of voting systems, ranging from completely physical voting systems to fully automated electronic systems that allow voting over the Internet. Completely physical systems typically employ paper ballots that a voter must deposit in a ballot box. Fully automated systems use a voting machine: the voter simply presses a button after which the machine stores her vote. Hybrid systems use both physical and digital techniques in realizing the voting process. For example voting is done with paper ballots, but these are counted using an optical scan machine.

Voting systems are particularly good case studies for three reasons: first the security requirements are high, especially for nation-wide elections. Obviously, there can be no democracy without a proper election process. Second, although the security of electronic voting (also known as "e-voting") is widely discussed in the scientific community, there is little scientific knowledge regarding the security of paper voting [230]. It is difficult to experiment with voting systems and much knowledge is only tacitly present in handbooks and laws. Thus, we have the ironic situation that the "gold standard" of paper voting is less well understood than its intended replacement e-voting, so that claims of superiority of one over the other are hard to substantiate. Third, in spite of all research, opinions still widely differ on the security of electronic voting. For example, after adopting electronic voting systems, both the Netherlands and Germany banned these machines because they could not comply with security requirements such as vote secrecy. As these two countries switched back to a paper-based approach [228], other countries such as Estonia still consider e-voting systems to be secure and continue to use them.

\footnotetext{
${ }^{1}$ This chapter is based on Van Cleeff et al [207].
} 
Computer society ACM took an intermediate position and stated in its communications that electronic voting machines should minimally be equipped with a paper audit trail to meet security requirements [78].

To understand the possibilities for automating voting, we will investigate the security characteristics of digital and physical voting processes further. Given the state of the debate, this chapter has two focus points. First, we intend to reverseengineer the physical voting process, and understand how it realizes security goals aided by the context. Second, we examine how far automation can be pushed to realize secure electronic voting.

We begin to examine background and related work in Section 7.2. Then Section 7.3 discusses our research method. Similar to the other case studies, we then perform reverse requirements engineering and extract security goals of voting in Section 7.4. We analyze how the steps in a different voting processes (paper, electronic and hybrid) contribute to or detract from these goals and investigate how to mitigate these obstacles in Sections 7.5, 7.7, 7.10 respectively. In between we answer our research questions. First in Section 7.6 and 7.8 we present an investigation of the role of the physical and digital entities in and we develop a typology of physical and digital entities with specific properties, and show how these contribute to security goals (RQ1 and RQ2). Using the requirements model, we compare electronic and paper voting in Section $7.9(\mathbf{R Q 3})$ and investigate the different types of hybrid voting systems (RQ4). We then consider the potential for hybrid voting systems in Section 7.11 (RQ5) . Given the controversy around electronic voting, we add a specific subquestion $\left(\mathbf{R Q 5} 5_{\text {Voting }}\right)$ concerning whether some for of electronic voting can be superior to the physical voting process. This is addressed in the last Section 7.12

\subsection{Conceptual Framework}

\subsubsection{Definition and Concepts}

Regardless of whether the voting is automated, there are two basic types of voting: kiosk voting and remote voting. In kiosk voting, the voter makes her choice inside a specific voting booth, typically located inside a polling station. In remote voting, the voter casts her vote outside of a polling station. A specific type of remote voting is proxy voting, where the voter authorizes someone to cast the vote because she is unable to do it herself (for example because of travel or illness). Other types of remote voting include sending one's vote by e-mail, fax or paper mail (also known as postal voting). 


\subsubsection{Security Assessment Methods}

Given the focus of this chapter on paper voting, we will now give a small overview of methods for investigating the security of physical voting processes, to place our own research into context ${ }^{2}$ First, formal methods exist that researchers can use to build models of physical processes, of which they can prove certain properties. Secondly, if exact modeling is not possible, simulations can be performed, taking into account the uncertainty that comes along with physical processes. Third, actual elections can be studied for strength and weaknesses, and these results can be generalized to other cases. We first summarize this literature and then comment on it in Section 7.2.3.

\section{Formal Models}

In the area of e-voting security, Weldemariam and Villafiorita propose a method for analyzing procedural security [225]. Procedures are actions executed by agents on assets that can belong to both to the digital and the physical domains. To this effect, they create UML activity models to represent procedures and describe possible actions on assets. These assets are classified according to their mobility, evolution and number of instances, and can be either digital or physical. They define threat actions such as replacement and removal on these assets. Next, they extend the model with threats and asset flows and define the security objectives. Finally, a model checker (NuSMV) is used to assess the security of the procedures.

In a similar approach, Bryl et al. evaluate procedural alternatives concerning the introduction of e-voting in Trentino, Italy [23]. Their objective is to mitigate the risk of introducing new security threats in newly developed electronic procedures, and use the existing paper procedures as a point of departure. To this end, they combine process modeling in UML (use case, activity and object diagrams) with goal-driven reasoning in the agent-oriented modeling tool Tropos. In particular, UML is applied for modeling both existing "as is" and proposed "to be" processes, while Tropos is used in between to assess the security of design alternatives, which the authors find hard to do in UML, both for providing a rationale for the chosen solutions, and for investigating security issues. The Tropos model is then transformed into a formal Datalog model, to automatically verify model properties.

\section{Simulations}

Pardue et al. advocate using simulation methods to determine the security of voting systems [160]. The first step is to create a threat tree: a hierarchical structure displaying the various means (starting with the leaves of the tree) that a threat

\footnotetext{
${ }^{2}$ This is an elaboration of the models discussed in Chapter 4
} 
(the root node) can be realized. Nodes are connected by special AND nodes (all leaves must be realized) or OR nodes (one of the leaves must be realized). Experts then estimate the likelihood of the leaves (called TERMINAL nodes), which are tied to the motivation of attackers and the complexity of the attack itself. Finally, Monte Carlo simulations can be used for doing risk assessment and performing trade-off analysis between specific systems, both paper and electronic.

\section{Election Observation}

Another source of information is rooted in the observation of existing (paperbased) elections and the study of actual attacks that took place, and the defenses that election officials implement to mitigate these threats. A very thorough discussion of paper voting was made by Harris [81] in his book on election administration in the United States. Knowledge about real elections is also codified in manuals for election observers, such as in use by the OSCE (Organization for Security and Co-operation in Europe). These typically follow a checklisting approach, where officials assess the security of an election process checking the status a long list of variables [155]-[157].

\subsubsection{Summary and Implications for Case Study}

As we already discussed in Chapter 4 on related work, model checking and simulation provide insight in the properties of election procedures, but in order to do so they abstract away from the enormous complexity of physical processes. It is not known which physical properties we can safely abstract away from for a security analysis, and which are important. Election observers also make abstractions, because they typically list the threats and mitigations, but fail to explain why these threats are actually possible, and therefore what can be done against them. What is missing is a systematic investigation of what physical properties are important to the voting process.

As an example, ballot theft is a well-known security problem of paper voting, as these ballots can be used to stuff the ballot box. Monitoring of the ballot box as well as strict voting ballot security can prevent this attack. However, this does not explain why such an attack is possible. Intuitively the attack is explainable by pointing out that the ballots must be manufactured prior to the voting, and lack any form of access control on them. In this chapter we will apply a more precise form of argumentation, to arrive at more well-founded conclusions. 


\subsection{Method}

Similar to our case studies on access control in Chapter 6, we will research three types of voting systems: digital systems, physical systems and hybrid systems and compare these concerning their ability to realize security goals.

\subsubsection{Case Study Selection and Method}

\section{Physical System}

For the physical system, we investigate the Dutch election for the European Parliament in 2009, in which 4.5 million people voted. The analysis is based on three sources:

- material from Dutch Election Council ${ }^{3}$ including legislation

- an interview with a Dutch election official

- the manual for election officials [137]

The interview took place on November 26th, 2009 and was held with the election coordinator of the municipality of Enschede. The interviewee was involved in organizing the European Elections that took place earlier that year on June 4th. During the interview, which was held at the city hall, in about one hour several topics were discussed:

- the exact procedures that took place during the election process

- the process of aggregating the results from different municipalities (this was not used for this research)

- the official's view on the overall security and complexity of the process

During this research, we were also given the aforementioned manual that was used for the election officials in the polling station, which exactly prescribed how the election should be held.

We had three reasons for choosing this case: the voting process was nationwide (with correspondingly high security requirements), it was completely paperbased, and data about the process was easily accessible.

\footnotetext{
${ }^{3}$ http://www.kiesraad.nl
} 


\section{Electronic System}

In our search for a purely electronic voting machine, we found two well documented-cases of electronic voting machines, namely the AccuVote-TS Voting Machine [55] and the ES3B [73], [143], [144]. Developed by Diebold, the AccuVote-TS has been widely used in the United States, with more than 33,000 machines being in service. The ES3B was developed by Nedap / Groenendaal and was used in the Netherlands, where 8000 machines served over $90 \%$ of voters in the 2006 local election. These cases suffer from two drawbacks. The first is that these machines still use physical procedures: the Diebold machine requires that the user physically inputs a smartcard before voting and the Nedap/Groenendaal machine uses a printed layout of the candidates rather than a monitor. Second, the design of both machines is outdated and proven to be insecure, and potentially newer and more secure machines exist. For example, Paul and Tanenbaum proposed a new voting machine based on TPM hardware [164] (this is thus actually a hybrid system). To compensate for these problems we synthesize these cases in an idealized voting machine, that is completely electronic and has been equipped with extensive security mechanisms.

\section{Hybrid System}

As hybrid cases, we consider several implementations, which each highlight specific security solutions as well as threats:

- ballot printer

- optical scan machine

- redundant system

- Scantegrity II (a hybrid system that uses invisible ink)

- pen system

Ballot printers translate the voter's push of a button or touchscreen into a precise mark on a paper ballot. These machines can thus eliminate some of the problems with traditional ballots, which users can fill in erroneously [99]. Optical scan machines can scan ballots automatically, and reduce the chance of human error during the counting process [44], [167]. Furthermore, redundant systems are possible: any step in the voting process can be complemented with a redundant electronic or physical process step. First, the paper ballots can be used to test the accuracy of the electronic voting machine. Second, electronic records can be used to verify that the paper records were counted correctly. The former are also called voter-verified paper audit trails (VVPAT) [71]. We also discuss a system using invisible ink called Scantegrity II [29]. Scantegrity II is also an example of 
an end-to-end (E2E) voting system. E2E systems allow voters to check whether their vote was included in the vote tally. This is not trivial, as the voter should not be able to prove her vote directly, as this would facilitate vote buying. Finally we examine a pen system. This system consists of a pen equipped with a small scanning device. Voters can mark their vote on the paper ballot as usual, but in addition to that the vote is also scanned and stored in a tallying machine [220].

\subsubsection{Limitations and Scope}

As for the types of voting, we limit our research by focusing on kiosk voting: democratic voting inside a polling station using a voting booth. First, this excludes remote voting, such as postal voting from the research. For a detailed investigation of such methods we refer to Puiggali and Morales-Rocha [169] and Krimmer and Volkamer [122]. Second, this excludes events not taking place on Election Day, such as the printing of ballots. Third, concerning electronic voting, we did not perform an in-depth investigation of systems that apply cryptographical solutions such as mix-nets for keeping votes secret. The main reason is that in our view, cryptographic solutions suffer from the same problems as the other electronic systems in this chapter: they rely on governments or other organizations to manage cryptographic keys and hardware and it is difficult to assure other parties that these systems are running as intended. Thus we chose not to investigate these systems further, also given our limited research budget.

\subsection{Goals}

Our case study of voting begins with the goals that a voting process should realize. Many different sets of goals have been put forward [114], [116], [169]. We include all of these goals and group them by information security properties: confidentiality, integrity and availability. Added to these we also add the goal of assurance, the confidence that these properties actually hold, which we believe to be crucial for accepting the election result.

Figure 7.1 shows the resulting goal tree. We will use this tree to explain precisely what security goals the operations in the voting process can help realize. Ultimately, voting processes (with their specific security goals) contribute to the top-level goal of allowing citizens with the right to vote to take part in the government, either directly or by being represented through a representative (T1) [155]. Two subgoals realize this: an election that satisfies all security goals (T2) and eligible voters actually voting in the election (T3). Except for the assurance goal (S1), all goal decompositions are AND decompositions. Below, we explain the goals in the tree in further detail. 


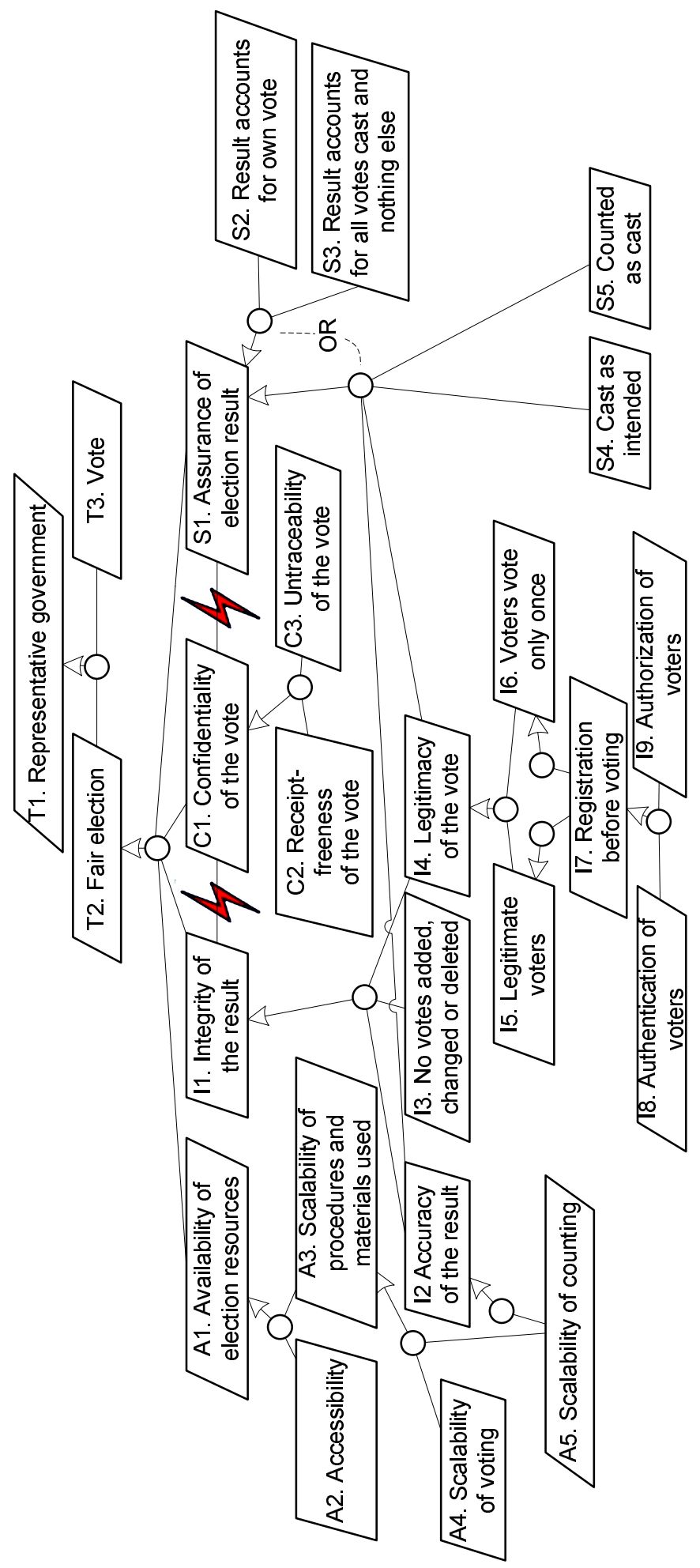

Figure 7.1: Top-level goal tree. The lightning symbol indicates a goal conflict. 


\subsubsection{Availability}

To allow voters to cast their vote, resources such as the ballot box and the voting booth need to be available (A1). Availability also entails that people with disabilities should be able to vote; this is the accessibility goal (A2). The procedures and resources used should be scalable (A3), as integrity is hard to achieve when the process takes longer [230], both regarding the voting itself (A4) and the counting (A5).

\subsubsection{Integrity}

The process between the start of the election and the announcement of the results must be executed correctly, such that all legitimate votes are represented in the end result. This is the goal of integrity (I1).

First, integrity requires legitimate votes (I4): only registered voters can vote (I5) and they vote only once (I6). Second, once votes are cast, it is impossible to add, change or delete votes (I3). Legitimacy is realized by a registration procedure before the voting (I7), in which voters are authenticated (I8) and their eligibility to vote is determined (I9). A separate goal is that of accuracy (I2): the extent to which the transformation of votes into final results occurs without errors.

\subsubsection{Confidentiality}

There are many possible ways to phrase the secrecy of the vote including "vote secrecy", "confidentiality of the vote", "voting by secret ballot". To be precise, what must remain confidential is not the vote itself but the relation between the voter and the vote. Obviously the vote must be counted in the end, and is not a secret itself. Thus we have the goal of Confidentiality (C1), which requires that the link between the vote and the voter is secret, and cannot be revealed by anyone, not even the voter herself. Confidentiality is realized in two ways: first, voters should keep their vote private, so that others cannot buy their votes, or can coerce them to vote for a particular candidate. We call this "receipt-freeness of the vote" $(\mathbf{C 3})$, i.e. the voter does not have a "receipt" to show how she voted. Second, others should not be able to deduce that a voter voted in a particular way. We call this "untraceability of the vote" $(\mathbf{C 2})$.

\subsubsection{Assurance}

All agents in the process (voters, candidates and election officials) must have confidence in the election outcome. Thus the goal of assurance must be realized (S1), which is the confidence that the election achieves the security goals. Achieving assurance is possible in two different ways ( $O R$ decomposition). 
The first approach is by ensuring confidence in each step:

- Legitimate voters: only eligible voters can vote (I4).

- Cast as intended: the votes are not changed after the moment of casting. (S4)

- Counted as cast: the vote count reflects all votes cast (S5) and the counting is accurate (I2).

The second approach is to ensure that results accounts for one's own vote (S2) and for all votes cast $(\mathbf{S 3})$.

\subsubsection{Goal Conflicts}

Solving goal conflicts is not trivial, as one goal's realization makes it more difficult to satisfy another goal. Thus, studying their mitigation yields important information. In our requirements model, the goal of confidentiality (C1) is at odds with the goals of integrity (I1) and assurance (S1) of the vote. The more strict the confidentiality goal is adhered to, the less data is available for checking the integrity of the entire process and vice versa. In one extreme case: if each voter gets a signed receipt of his vote, and these receipts are publicly made available, the integrity of the election is easily assured, at the cost of a complete loss of confdentiality.

Having establishing the goals in Section 7.4, we now examine physical, hybrid and electronic voting systems. For each, this results in a list of entities and operations, which contribute to the voting goals. All the models listed below pass the completeness checks in KAOS for a model: (i) all goals are supported by operations, and (ii) all operations should contribute to a goal. We begin to create the detailed KAOS model for the paper voting process.

\subsection{Paper Voting}

\subsubsection{Entities}

Figure 7.2 shows all the relevant entities in the election process such as ballots and pencils.

\subsubsection{Operations}

Figure 7.3 shows all the steps in the voting process as operations in KAOS, how they contribute to security, and their relations to the actors and entities. The next paragraphs describe the operations that take place in further detail. 


\begin{tabular}{rlrl}
\hline ID & Name & ID & Name \\
\hline E1 & Voter & E11 & Election report \\
E2 & Election official & E12 & Applicable laws \\
E3 & Polling station & E13 & Brochure valid IDs \\
E4 & Voting booths & E14 & Voting manuals \\
E5 & Ballot box & E15 & Packages with seals \\
E6 & Red pencil & E16 & Register cancelled vote cards \\
E7 & Pen & E17 & Candidate list \\
E8 & Stamp & E18 & Voter IDs \\
E9 & Ballots & E19 & Vote cards \\
E10 & Papers for tally marking & & \\
\hline
\end{tabular}

Figure 7.2: Entities used in paper voting (E1 and E2 are also agents).

\begin{tabular}{|c|c|c|c|c|}
\hline ID & Operation & Goal & Agent & Entities \\
\hline $\mathrm{P} 1$ & Open polling station & $\mathrm{S} 1, \mathrm{~A} 2$ & Election official & $\begin{array}{l}\text { Polling } \\
\text { station }\end{array}$ \\
\hline $\mathrm{P} 2$ & $\begin{array}{l}\text { Open and close } \\
\text { ballot box }\end{array}$ & $\mathrm{I} 3$ & Election official & Ballot box \\
\hline P3 & Seal ballot box & $\mathrm{S} 1, \mathrm{I} 3$ & Election official & Ballot box \\
\hline P4 & Open ballot box & $\mathrm{S} 1, \mathrm{~T} 3$ & Election official & Ballot box \\
\hline P5 & Close polling station & S1 & Election official & $\begin{array}{l}\text { Polling } \\
\text { station }\end{array}$ \\
\hline P6 & $\begin{array}{l}\text { Seal election } \\
\text { material }\end{array}$ & $\mathrm{I} 3, \mathrm{~S} 3, \mathrm{~S} 5$ & Election official & $\begin{array}{l}\text { Vote cards, } \\
\text { ballots }\end{array}$ \\
\hline P7 & Count ballots cast & A5, I1 & Election official & Ballot \\
\hline P8 & $\begin{array}{l}\text { Write down election } \\
\text { result }\end{array}$ & $\mathrm{S} 1, \mathrm{I} 1$ & Election official & $\begin{array}{l}\text { Election } \\
\text { report }\end{array}$ \\
\hline P9 & Enter polling station & T3, S1 & Voter & $\begin{array}{l}\text { Polling } \\
\text { station }\end{array}$ \\
\hline P10 & Hand over ID & I8 & Voter & Voter ID \\
\hline P11 & Hand over vote card & I9, I6 & Voter & $\begin{array}{l}\text { Vote card, } \\
\text { register } \\
\text { cancelled } \\
\text { vote cards }\end{array}$ \\
\hline $\mathrm{P} 12$ & Authenticate voter & I8 & Election official & Voter ID \\
\hline P13 & Authorize voter & I9 & Election official & Vote card \\
\hline P14 & Take in vote card & I9, I6 & Election official & Vote card \\
\hline $\mathrm{P} 15$ & Receive ballot & $\mathrm{T} 3, \mathrm{C} 3$ & Voter & Ballot \\
\hline P16 & Enter voting booth & $\mathrm{C} 2$ & Voter & Voting booth \\
\hline P17 & Inscribe ballot & $\mathrm{T} 3$ & Voter & Ballot \\
\hline P18 & Fold ballot & $\mathrm{C} 1$ & Voter & Ballot \\
\hline
\end{tabular}




\begin{tabular}{rllll}
\hline ID & Operation & Goal & Agent & Entities \\
\hline P19 & Deposit ballot & T3, S4 & Voter & Ballot \\
P20 & Exit voting booth & T3 & Voter & Voting booth \\
\hline
\end{tabular}

Figure 7.3: Operations performed by agents on entities, and their contribution to goals.

The Election Day The Election Day starts with the arrival of the election officials (E2). Before opening of the polling station (E3), the chair of the committee checks the arrangement of the polling station, which must satisfy five requirements:

- No one can see someone marking her vote.

- Access to booths is visible for voters and officials.

- Voters are able to see the actions of officials.

- Voters (who are standing in line before voting) do not obstruct the view on other voters.

- The ballot box is within reach of an official that checks whether the voter casts his ballot.

Specific documents should be present: including copies of applicable laws (E12), a brochure on how to recognize valid IDs (E13), manuals for the voters $(\mathbf{E 1 4})$, the list of candidates (E17), the ballots and the election report that has to be filled in during the day. The officials verify that the ballot box is empty, closed and sealed $(\mathbf{P 2}, \mathbf{P 3}, \mathbf{P 4})$. Next, they count the available ballots (E15) and put the number in the election report.

Actual Voting Procedure The voter (E1) enters the polling station (P9), identifies herself with an ID (such as a driver's license) to the election officials (P10) and hands over the vote card (P11,E19), a unique paper card with water mark that is distributed to the voters prior to the elections.

Next, the election officials check the voter's ID (E18), whether it is still valid (E16), and whether it corresponds to the voter's appearance. The officials check the vote card for its authenticity (P12) and put a stamp (E8) on it, so that it is invalid for other voters (P14). They then remove a ballot (E9) from a stack of ballots and hand it out to the voter (P13,P15), tallying how many are handed out.

The voter subsequently enters the voting booth $(\mathbf{P 1 6}, \mathbf{E 4})$ and uses a red pencil (E6) to choose a candidate (P17), folds the ballot (P18), exits the voting booth 


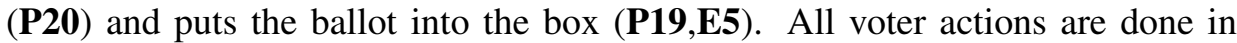
plain sight of the officials, who keep track of how many ballots are deposited. Finally, the voter leaves the polling station.

When the voting period is over, the officials close the ballot box and write down the number of votes cast and the number of ballots handed out in the election report using a pen $(\mathbf{P 5}, \mathbf{E 7})$. Vote cards, unused ballots and returned (invalidated ballots) are put into separate packages which are sealed (P6). The officials open the ballot box and tally the ballots (P7,E10). The number of ballots counted should be equal to the number of vote cards minus the ballots that were not deposited by the voters. Again, the numbers are written down and announced in public. If the numbers do not match the ballots are counted again; if necessary the packages are opened.

Next the ballots are unfolded and sorted by political party, separating blanks and invalid ballots. The officials show invalid ballots to observers present in the polling station. The final count is done by candidate. Any complaints are noted in the report and the packages with the votes are sealed (P8). Finally, the officials sign the election report (E11) and deliver it to the municipality.

\subsubsection{Threats and Mitigations}

Threats against the paper voting process can be found in many sources, among those literature on e-voting [25], [113], [149], [213], and election manuals [137], [157]. Specific paper voting threats are listed by Jones [113] and Harris [81]. We select threats on the basis of three criteria: (i) they occur on Election Day, (ii) they are relevant for the paper voting process, and (iii) threats are non-violent. Similarly to the goal tree, we group these threats based on the top-level security properties. The main threats are summarized in Figure 7.4 .

\section{Confidentiality Threats}

The first threat to the confidentiality of the vote is to mark the ballots (T1) such that they are traceable to the voter, for example by leaving fingerprints. This is mitigated by securely storing and destroying the ballots. Still, voters can either mark the ballots themselves (such as by voting in a unique pattern [29]), or others can pre-mark ballots, such as by having unique serial numbers.

The voting itself can also be recorded (T2), for example by using a smartphone camera [113] or by forcing voters to accept "assistance" in the voting booth. The voter can also simply show his ballot in public before casting it. Regulation can mitigate these threats by forbidding such actions, or declaring such votes to be invalid. Chain voting (T3) is a specific threat in which a vote buyer hands a prefilled ballot over to a voter, who casts it and delivers the blank ballot (which the voter received from the officials) in return, allowing the vote buyer to continue 


\begin{tabular}{rll}
\hline ID & Threat & Threat to Goal \\
\hline T1 & Marking ballots & $\mathrm{C} 3$ \\
T2 & Recording the vote & $\mathrm{C} 2$ \\
T3 & Chain voting & $\mathrm{C} 2$ \\
T4 & Unauthorized voting & $\mathrm{I} 8, \mathrm{I} 9$ \\
T5 & Adding, removing and changing ballots & $\mathrm{I} 2, \mathrm{I} 3$ \\
T6 & Improper counting & $\mathrm{I} 2, \mathrm{I} 3$ \\
T7 & Incorrect proclamation of results & $\mathrm{I} 2, \mathrm{I} 3$ \\
T8 & Improper or chaotic procedures & $\mathrm{I} 2, \mathrm{I} 3$ \\
T9 & Denying possibility to vote & $\mathrm{A} 3$ \\
T10 & Inability to observe the process & $\mathrm{S} 1$ \\
T11 & Inability to interpret the process & $\mathrm{S} 2, \mathrm{~S} 3, \mathrm{~S} 5$ \\
T12 & Inadequate management of complaints & $\mathrm{S} 2, \mathrm{~S} 3, \mathrm{~S} 5$ \\
\hline
\end{tabular}

Figure 7.4: Threats to voting goals for paper voting.

the "chain" [111]. Mitigating chain voting is done by marking ballots on handout and checking the mark on deposit. Other mitigations are to allow the ballots to be spoiled, and to enforce strict ballot security.

\section{Integrity Threats}

A general integrity threat is chaotic or improper bookkeeping and logistics (T8). For example, ballot box stuffing is made easier by not keeping track of how many ballots are cast. Procedures should define exactly where and how many ballots are stored and how the voting process occurs. Threats against integrity occur at each step:

During the Authentication and Authorization of the Voters Threats include tampering with the vote registry, not performing authentication and authorization and not keeping track of who voted (T4). Mitigations include distributing unique authorization documents to voters, which are taken in by the officials on ballot handout.

During the Voting Itself Two threats are stuffing the ballot box with votes, or even swapping the whole box for another one (T5). Other threats are that voters receive more than one ballot, that cast votes are altered or that votes are removed from the ballot box, or substituted by others. Sealing the votes, having observers in the polling station and comparing records (how many people registered, how many voted) mitigate these threats. 
During the Counting of the Votes Votes can be miscounted (or not counting at all) (T6). Prevention includes counting the votes in public, and having clear rules for invalidating or judging ballots. Because ballots are touched during counting, officials can change them, which is hard to detect ${ }^{4}$ As counting usually takes place in multiple stages, it can be checked whether the totals for each stage are equal. If they are not, that is an indication of fraud. [112]

During the Proclamation of the Results Threats include not announcing an official and public notice of the final results (T7) [157, p.64]. Alternatively, there can be a final result, which is altered later, for example by filling an election report by pencil instead of pen. Delays in the proclamation of the results give the opportunity to falsify election data: the threat is mitigated when the results are available as soon as the counting is finished.

\section{Availability Threats}

If the polling station is not available and people cannot vote, the election is unfair. Threats to the ability to vote (T9) are long waiting queues, insufficient ballots or an early closure of the polling station.

\section{Assurance Threats}

Observing the election process gives confidence in the election result. This leads to three categories of threats:

First there is the inability to observe the voting process (T10) by being denied access to the polling station, being unable to observer the voting, counting, or oppositely, or because activities such as finalizing the results are done outside the polling station.

Secondly, participants need to be able to interpret the voting process (T11). If the protocol is not available to the observers, is incomplete, inconsistent or is changed the observers lack assurance. This is also the case if the observers miss the necessary expertise to understand the protocol.

Thirdly, observations of problems need to be managed properly (T12). The election process must be able to deal with small problems that arise, or make corrections, without discrediting the whole election.

\subsection{Properties of Physical Entities}

We will now attempt to discover general physical domain properties contributing to security, using the method explained in Chapter 5. First Figure 7.5 shows

\footnotetext{
${ }^{4}$ Counters can put a small piece of pencil lead (“short pencil”) under a fingernail |192|.
} 
the support for the existing hypothesis in the voting case study. After that, we explain the new hypothesis that were identified (Figure 7.6).

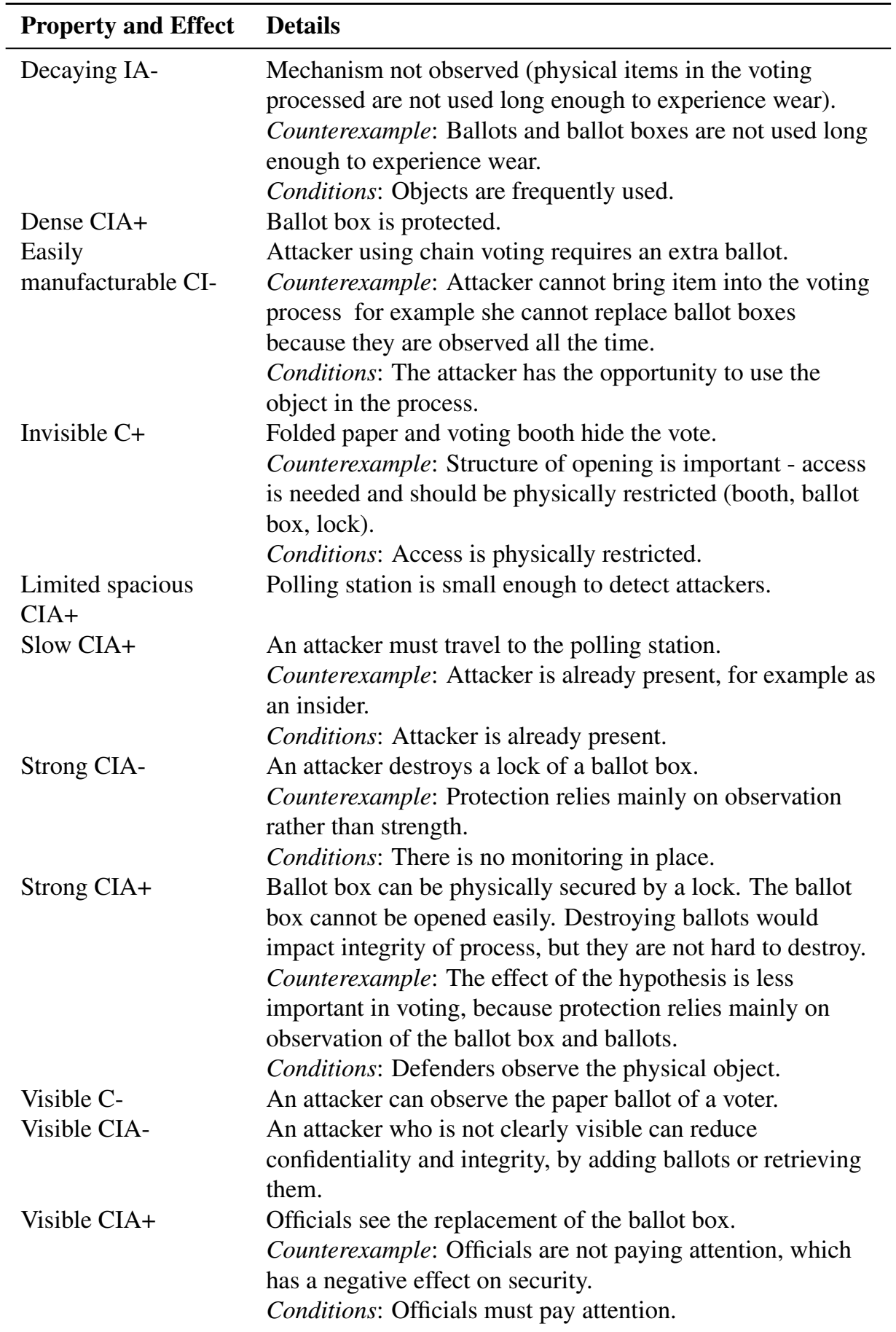




\begin{tabular}{ll}
\hline Property and Effect & Details \\
\hline Weak CIA+ & $\begin{array}{l}\text { Attacker cannot easily open a ballot box. } \\
\text { Counterexample: Attacker can unfold ballot, as this is } \\
\text { observed. } \\
\text { Conditions: Physical barrier is present. }\end{array}$ \\
\hline
\end{tabular}

Figure 7.5: Support for existing hypotheses (consisting of properties and their effects) in the voting case study. If counterexamples lead to new hypotheses they are listed individually in Figure 7.6 .

Active A person executes a process with security tasks and intervenes when something goes wrong, improving overall security. An example specific to this case is that a person votes. A counterexample is that an attacker opens the ballot box. This leads to one condition:

- The person is malicious.

This leads to the alternative hypothesis namely that an active person can also have a negative effect on overall security. An example specific to this case is that an attacker steals ballots. A counterexample is that the person is not malicious. This leads to one condition:

- The person is not malicious.

Difficultly Manufacturable When objects are difficult to manufacture this increases the confidentiality and integrity of these objects. A contributing factor is when these entities are used in a controlled space such that no manufacturing equipment or raw materials can be brought in without detection. An example specific to this case is that it is not possible to simply create a new paper, or ballot or filled in ballot.

Enclosed With Opening A closed physical structure with an opening creates an opportunity for confidentiality because a person can enter it and perform actions inside without being observed inside. An example specific to this case is that a voting booth shields voter and ballot against attackers looking at them. A counterexample is that the voter can execute illicit tasks such as chain voting because she is not observed inside the voting booth. This leads to two conditions:

- Person is not malicious.

- Persons are screened for reliability. 


\begin{tabular}{|c|c|c|}
\hline Property & Effect & Hypothesis \\
\hline Active & $\mathrm{CIA}+$ & A person intervenes in a process to increase its security. \\
\hline Active & CIA- & An attacker disrupts a process. \\
\hline $\begin{array}{l}\text { Difficultly } \\
\text { manufacturable }\end{array}$ & $\mathrm{CI}+$ & $\begin{array}{l}\text { A difficultly manufacturable object is hard to create or } \\
\text { alter precisely. }\end{array}$ \\
\hline $\begin{array}{l}\text { Enclosed with } \\
\text { opening }\end{array}$ & $\mathrm{C}+$ & $\begin{array}{l}\text { An enclosure with opening allows unobserved access to } \\
\text { the confidential information stored inside. }\end{array}$ \\
\hline Inert & $\mathrm{CIA}+$ & $\begin{array}{l}\text { An inert object cannot alter or expose itself or make } \\
\text { itself unusable. }\end{array}$ \\
\hline Large & $\mathrm{I}+$ & An attacker cannot hide a large object. \\
\hline Modifiable & $\mathrm{CI}+$ & $\begin{array}{l}\text { Persons modify entities as part of the normal business } \\
\text { processes. }\end{array}$ \\
\hline Modifiable & CI- & An attacker modifies entities and changes their state. \\
\hline Movable & $\mathrm{CIA}+$ & $\begin{array}{l}\text { Movement of persons and objects is necessary for } \\
\text { executing a process. }\end{array}$ \\
\hline Movable & CIA- & An attacker disrupts a process by moving objects. \\
\hline
\end{tabular}

Figure 7.6: New hypotheses from the voting case study about the security effects of physical properties of entities. Each row represents either positive or negative effects. Multiple letters indicate an effect on multiple security goals. For example CIA- means a negative effect on confidentiality, integrity and availability.

Inert An inert object by itself cannot steal or reveal information (confidentiality), nor alter its state (integrity), nor change into an unusable form (availability) without another actor such as a person being involved. An example specific to this case is that a ballot box remains unchanged and the ballots inside it cannot change by themselves. There are two conditions:

- There is no wear.

- The object does not have autonomous capabilities.

Large It is difficult to smuggle a large object because it is observable and difficult to carry. Thus the large size of an object has a positive effect on integrity. An example specific to this case is that smuggling of ballots is necessary to realize the threat of chain voting. A counterexample is that other items than ballots can be brought in the process but these are not used or are not limited and therefore do not impact security. This leads to two conditions:

- The object is used in the process.

- The process depends on unique objects. 
Modifiable Normal modification is part of a business process and helps confidentiality and integrity. An example specific to this case is that a person can fold a ballot and write on it. However modification also allows attacker to execute attacks against the integrity and confidentiality of an object. An example specific to this case is that modification of ballots is easy (changing the ballot and marking it). A counterexample is that the attacker is observed while performing the modifications. This leads to one condition:

- The attacker is able to modify objects unobserved.

Movable Movement of persons and items is part of the process, contributing to security in general. An example specific to this case is that a person moves a ballot. A counterexample is that the attacker steals a ballot. This leads to one condition:

- The person is not malicious.

However an attacker can also move objects, impacting security negatively. An example specific to this case is an attacker that puts an extra ballot in the ballot box.

\subsection{Electronic Voting}

Figure 7.7 shows the new entities (compared to paper voting) that are necessary in our ideal model of electronic voting. Note that some entities for the paper voting such as red pencils are no longer necessary.

\subsubsection{Entities}

The actual voting takes place on the voting machine, which is equipped with a display and a touch screen. Inside our ideal e-voting machine is a processor and flash memory from which the system is booted. The poll workers have access to the voting server, which has a small display and a button interface. On the voting server runs software that can collect the information from the different voting machines and print the results. A printer will output the final results. All systems are connected via cables. Removable memory cartridges are used for installation of the voting machine software and for the definition of the election.

\subsubsection{Operations}

We will now briefly describe the procedure for our idealized electronic voting machine, which is primarily based on the AccuVote-TS Voting Machine [55]. The description concentrates on the interaction with the voting machine and leaves out 


\begin{aligned} & \hline ID Name \\ & \hline E6 Red pencit \\ & E9 Ballots \\ & E10 Papers for tally marking \\ & E15 Packages with seals \\ & E20 Voting machine \\ & E21 Voting server \\ & E22 Printer \\ & E23 Cables \\ & E24 Memory cartridges \\ & E25 Voting machine software \\ & E26 Voting server software \\ & \hline\end{aligned}

Figure 7.7: Additional entities used for electronic voting.

details that were presented earlier for the paper voting case. For example we will not re-explain the opening and closing of the polling station and the sealing of election material. The steps are shown as KAOS operations in Figure 7.8. On Election Day, an empty memory cartridge is placed into the voting machine and it is booted (P1). The machine is now in PreDownload mode and the poll workers insert the memory cartridge with the election definition (P2). The machine enters the PreElectionTesting mode and the poll workers test the working of the machine (P3). Next the poll-workers configure the machine to be in Election mode (P4). On the screen the totals are displayed, which are all zero (P5). The voting can now begin.

The voter first identifies herself as described in the paper voting section (P6). Next the chairman of the election committee releases the machine for voting by clicking a button (P7). After entering the voting booth the voter selects a candidate on the screen (P8). The machine gives off a beep (P9). Next, she clicks the vote button $(\mathbf{P 1 0})$ and the voting machine stores the votes (P11). In her display the voter receives the message that she has voted, and the same message appears on the server (P12). After the election, the committee closes the election by inserting another cartridge (P13). The machine is now in PostElection mode. It transfers the results to the server $(\mathbf{P 1 4})$, which prints the election results $(\mathbf{P 1 5})$.

\subsubsection{Threats and Mitigations}

For electronic voting, we first list all threats found, and then return to potential mitigations to these threats.

Threats Figure 7.9 shows a high-level list of threats and we explain them in detail below. 


\begin{tabular}{|c|c|c|c|c|}
\hline ID & Operation & Goal & Agent & Entities \\
\hline $\mathrm{P} 1$ & $\begin{array}{l}\text { Place empty cartridge in } \\
\text { machine }\end{array}$ & A & Election official & $\begin{array}{l}\text { Voting machine, } \\
\text { cartridge }\end{array}$ \\
\hline $\mathrm{P} 2$ & $\begin{array}{l}\text { Insert cartridge with } \\
\text { election definition }\end{array}$ & A & Election official & $\begin{array}{l}\text { Voting machine, } \\
\text { cartridge }\end{array}$ \\
\hline P3 & $\begin{array}{l}\text { Do logical and accuracy } \\
\text { testing }\end{array}$ & I & Election official & Voting machine \\
\hline $\mathrm{P} 4$ & $\begin{array}{l}\text { Set machine in Election } \\
\text { mode }\end{array}$ & A & Election official & Voting machine \\
\hline P5 & Display initial totals & A & Voting machine & \\
\hline P6 & $\begin{array}{l}\text { Authenticate and authorize } \\
\text { voter }\end{array}$ & I & Voter & Election official \\
\hline P7 & Release machine for voting & A & Election official & $\begin{array}{l}\text { Voting server, voting } \\
\text { machine }\end{array}$ \\
\hline P8 & Selects candidate & $\mathrm{A}$ & Voter & Voting machine \\
\hline P9 & Sound beep & A & Voting machine & \\
\hline $\mathrm{P} 10$ & Click vote button & A & Voter & Election official \\
\hline $\mathrm{P} 11$ & Store vote & CIA & Voting machine & \\
\hline P12 & Display "voted", & A & Voting machine & Voting server \\
\hline P13 & Inserting end cartridge & A & Election official & $\begin{array}{l}\text { Voting machine, } \\
\text { cartridge }\end{array}$ \\
\hline P14 & $\begin{array}{l}\text { Transfer results to voting } \\
\text { server }\end{array}$ & A & Voting machine & Voting server \\
\hline P15 & Print election results & A & Voting server & Printer \\
\hline
\end{tabular}

Figure 7.8: Operations performed by agents and their contribution to goals.

Replace Software A first attack is to replace the software with a malicious version (T13). There are three main ways to realize this. The first is to open the machine and replace the flash memory inside it. This requires bypassing physical security, removing screws and replacing the chips containing the software. The second is to simply reprogram the machine by putting it on and inserting a cartridge that can update the software. This does not need to be done by the attacker herself, as the cartridge could contain a virus that can propagate along voting machines, as each of them are subsequently updated with the new election files. Replacing the software affects all security properties.

Misuse Existing Software It can also be possible to misuse the software [144], if a bug is present (T14). In our case, the attacker is an official who changes the mode of the machine back to Maintenance. Just before the voter casts the vote (selecting a candidate is accompanied by an audible beep), the attacker briefly puts the machine into maintenance mode by pressing a button on the server. After that, the vote is not recorded but the voter sees a message that her vote has been cast. 


\begin{tabular}{cll}
\hline ID & Threat & Goal \\
\hline T13 & Replace software & CIAS \\
T14 & Misuse existing software & I \\
T15 & Use faulty software & CIAS \\
T16 & Recover forensic data & C \\
T17 & Tamper with seals and locks & AS \\
T18 & Monitor radio emanations & C \\
\hline
\end{tabular}

Figure 7.9: Threats to voting goals for electronic voting.

At the end of Election Day, the attacker casts as many votes herself as necessary to make the records correct. This is an attack against the integrity of the system.

Use Faulty Software Another threat is that the official voting software itself has bugs, which might prevent a correct counting of the results (T15). Experiments have shown that even simple election software programs can have behavior that is hard to understand. Bugs can threaten the integrity of the election and also availability of the result: if the results are visibly incorrect (the numbers do not add up) then the election result is declared invalid.

Recover Forensic Data Inside the machine, the votes should be stored in such a way that an attacker cannot determine at what time the vote was cast. Thus, the ballots must be stored in random memory locations. If this is not done properly, an attack with access to the voting machines memory can deduce the time of the vote and correlate this with the voter's entrance in the voting booth (T16).

Tamper with Seals and Locks To prevent tampering with the machine, seals and locks can be put on the voting machine. This also implies that a broken seal or lock requires action from the election officials, who must test whether the machine's security has not been breached. Thus tampering with seals is an effective denial-of service attack (T17).

Monitor Radio Emanations As any electronic device, a voting computer sends out radio emanations. An attacker can use these to reconstruct the activities of the voting machine, and thus violate the secrecy of the vote (T18).

Mitigations We will now list mitigations for the threats listed before and explain their strengths and weaknesses. 
Physical and Procedural Security Physical security is the first defense against accessing modifying the machine's hardware and software. First the machine can be equipped with seals and locks. In practice these seals are often broken and the locks are easily circumvented. Furthermore, after a seal is placed, no further checks can be done, which requires absolute trust into the person that places the seal. Second the machine should be placed in a secured location and poll workers must secure all components of the voting machine, ensuring a proper chain of custody. However, in practice poll workers have unsupervised access to the machine, for example they are stored in the poll worker's home (a so-called "sleepover").

Software Testing Software testing can be used to find bugs in the voting software. There are two particular problems with software testing. The first is that software correctness is hard to ensure. Experiments have demonstrated this [11]. Second, the software verification is done by third parties but the independence of these investigations is questionable, as these parties (independent test authorities) are paid for and report to the machine's manufacturer.

Secure Booting To ensure that the correct software is running, the voting machine should check the signature of the programs at boot time. TPM (trusted platform module) hardware exists for this purpose. A potential circumvention is to replace the chip that contains the program with a programmable emulator: at boot time it will output the correct program, but the contents will change when an actual election is run.

A TPM-enabled voting machine is proposed by Paul and Tanenbaum [164]. This solution requires full trust in the TPM hardware manufacturer. Especially if this is a foreign company this might be unacceptable. Furthermore, their solution is still vulnerable to an attacker that adds hardware. When a user attempts to verify that a specific software is running on the machine, she can actually only verify that this software is running on hardware that is present in or connected to the machine. She cannot verify the absence of other hardware or software using this procedure. This leads to the issue of assuring that voting machine has the right hardware:

- Is the required hardware present on the machine? TPM solutions can assure this, if we trust the manufacturer.

- Is no other hardware running on the machine? This requires a detailed physical examination of the device.

One partial solution is to use transparent casing; any addition to the required hardware would then be easier to detect. In some cases this is more difficult because an attacker can for example add hardware under a touch screen. 
Parallel Testing Prior to an election, election officials can test the voting machines using so-called parallel testing: they simulate that an election is running on a number of voting machines and test these simultaneously. If the results are correct, the machines are expected to work properly. However, this mitigation can be circumvented, because the malicious software can distinguish a test (where it should function honestly) from the actual election (that should be rigged):

- The election will likely take much longer than the testing.

- The testing procedure will likely not be a random distribution of votes as in a real election.

- The malicious code is not running unless activated (for example, in an actual election, the attacker will tap on the device's input in a specific way, a socalled "secret knock").

Forensic Analysis In case the machine keeps multiple records and audit trails, forensic analysis might yield additional information beyond the votes themselves that can be used to find signs of malicious software activity. However, if a malicious program has access to all records, it might escape detection. Furthermore, forensic analysis of the records might leave additional hardware undetected, as it is transparent to both the system and the user.

Monitor Radio Emanations Mitigations against radio emanations include shielding the equipment by special cables and casings. The drawback is that these must be tested and can become very large and heavy.

\subsection{Properties of Digital Entities}

Figure 7.10 shows the support for the existing hypothesis, and Figure 7.11 lists the new hypothesis about the impact of digital properties on security. 


\begin{tabular}{ll}
\hline Property and Effect & Details \\
\hline Accurate Data Storage, & Accuracy helps to ensure that the votes are \\
Transportation and Processing & correctly captured and counted and not lost. \\
CIA+ & Counterexample: Accuracy of storage and \\
& processing makes it easy to forge votes (lack of \\
& uniqueness), having a negative impact on \\
& integrity. \\
\hline
\end{tabular}

Figure 7.10: Support for existing hypotheses (consisting of properties and their effects) in the voting case study. If counterexamples lead to new hypotheses they are listed individually in Figure 7.11 .

Accurate Data Storage, Transportation And Processing Accuracy storage, transportation and processing of data makes it easier for an attacker to forge records and thus threatens integrity. An example specific to this case study is that digital records of votes can be changed very precisely such that they are indistinguishable from the original records.

Fast Data Processing Fast processing of data makes abuse fast, having a negative effect on data availability because the attacker can alter and destroy it. An example specific to this case study is that a system can be stopped easily. There is one condition:

- The system is compromised or compromisable.

Fast Data Storage Fast data storage makes it easy to share confidential information. An example specific to this case study is a system that can store more data than it is supposed to, saving confidential information that was not supposed to be stored. There is one condition:

- The system is compromised or compromisable.

Program Computational Complexity The computational complexity of programs increases the chance of errors and abuse thus having a negative impact on security in general. Specifically, understanding what software exactly does is difficult. An example specific to this case study is that understanding what software exactly does is difficult (for example changing votes, or exposing voters), and small mistakes can lead to large errors or vulnerabilities. 


\begin{tabular}{lll}
\hline Property & Effect & Hypothesis \\
\hline $\begin{array}{l}\text { Accurate Data Storage, } \\
\text { Transportation and Processing }\end{array}$ & I- & $\begin{array}{l}\text { Accuracy makes record forgery } \\
\text { easy. }\end{array}$ \\
Fast Data Processing & A- & $\begin{array}{l}\text { Fast modification of data makes } \\
\text { abuse easy. }\end{array}$ \\
Fast Data Storage & C- & $\begin{array}{l}\text { Fast data storage makes sharing } \\
\text { confidential information easy. } \\
\text { System complexity increases the } \\
\text { chance of errors and abuse. }\end{array}$ \\
\hline
\end{tabular}

Figure 7.11: New hypotheses from the voting case study about the security effects of digital properties of entities. Each row represents either positive or negative effects. Multiple letters indicate an effect on multiple security goals. For example CIA- means a negative effect on confidentiality, integrity and availability.

\subsection{Comparison between Paper and Electronic Voting}

We will now compare physical and electronic forms of voting to find similarities and differences. To begin, we investigate the differences between physical and electronic entities. Next we compare physical and electronic systems as a whole on their ability to realize security goals.

\subsubsection{Comparison between Entities}

First, we compare the properties of a digital ballot to those of a paper ballot. On analysis, a paper ballot serves several functions:

- It presents the candidates to the voters (that are printed on the paper).

- It stores the voter's choice (the mark on the paper).

- It masks the voter's choice by the folding.

It is clear that the voting machine is an intermediate in every step of the interaction: electronic ballots are presented on a screen, manipulated via a touch interface, stored inside the voting machine and counted by the voting machine. As such the electronic entity of ballot does not exist individually.

Second, we compare the ballot boxes. The deposit into the ballot box of the ballot is not observable in electronic voting. Furthermore, the bandwidth of a physical ballot box is inherently limited (due to its size, it is not possible to add 100 ballots at the same time). By contrast, the electronic ballot box has no such restriction. Attack software can write thousands of votes to the storage location instantly [73]. 


\begin{tabular}{|c|c|c|c|}
\hline Security Goal & Effect & Physical Property & Digital Property \\
\hline All & + & $\begin{array}{l}\text { Active, Dense, Inert, } \\
\text { Limited spacious, Movable, } \\
\text { Slow, Strong, Visible, Weak } \\
\text { Active, Movable, Strong, } \\
\text { Visible }\end{array}$ & $\begin{array}{l}\text { Accurate Data Storage, } \\
\text { Transportation and } \\
\text { Processing } \\
\text { Program Computational } \\
\text { Complexity }\end{array}$ \\
\hline \multirow[t]{2}{*}{ Confidentiality } & + & $\begin{array}{l}\text { Difficultly manufacturable, } \\
\text { Enclosed with opening, } \\
\text { Invisible, Modifiable }\end{array}$ & $\mathrm{N} / \mathrm{A}$ \\
\hline & - & $\begin{array}{l}\text { Easily manufacturable, } \\
\text { Modifiable, Visible }\end{array}$ & Fast Data Storage \\
\hline \multirow[t]{2}{*}{ Integrity } & + & $\begin{array}{l}\text { Difficultly manufacturable, } \\
\text { Large, Modifiable }\end{array}$ & N/A \\
\hline & - & $\begin{array}{l}\text { Easily manufacturable, } \\
\text { Modifiable }\end{array}$ & $\begin{array}{l}\text { Accurate Data Storage, } \\
\text { Transportation and } \\
\text { Processing }\end{array}$ \\
\hline Availability & - & N/A & Fast Data Processing \\
\hline
\end{tabular}

Figure 7.12: Comparison of physical and digital properties on their ability to realize security goals for the voting case study. This table is constructed from data presented in Figures 7.5, 7.6, 7.10 and 7.11.

\subsubsection{Comparison Concerning Goal Realization}

Figures 7.12 and 7.13 summarize the mechanisms present in physical and digital voting. These are explained below.

Confidentiality For paper voting, confidentiality is realized because the entities are inert. This is not the case for an electronic voting system that is actively running software and can keep track of the history of events that can be used to link vote and voter. For electronic voting, a positive contribution is that attackers cannot easily observe votes, as they are stored hidden inside the machine's memory.

Integrity Paper voting integrity is realized because voting processes are slow. Therefore errors are detectable by persons inside the polling station. Furthermore, this slowness also limits the speed at which an attack can take place. For electronic voting, the precision made possible by the voting machine has a positive effect on integrity. At the same time, it is hard to ensure that the correct program is running on that machine, due its volatile nature. This has a negative impact on integrity. 


\begin{tabular}{lll}
\hline Goal & Physical Voting System & $\begin{array}{l}\text { Electronic Voting } \\
\text { System }\end{array}$ \\
\hline Confidentiality & + inertness of entities & $\begin{array}{l}\text { + hidden storage of } \\
\text { information } \\
\text { - possibility to keep } \\
\text { track of history } \\
+ \text { precision of } \\
\text { processing }\end{array}$ \\
& + slowness of process & $\begin{array}{l}\text { - volatility of software } \\
\text { and data } \\
\text { Integrity }\end{array}$ \\
& + volatility of software \\
& and data \\
Availability & + inertness of entities & $\begin{array}{l}\text { - volatility of software } \\
\text { and data }\end{array}$ \\
\hline
\end{tabular}

Figure 7.13: Comparison between physical and digital voting systems. "+” and "-" indicate positive and negative effects. The explanation of the scores for each goal and system is given in Section 7.9.2.

Availability Also availability benefits from the inertness of entities in paper voting, and again by contrast entities in electronic voting are volatile. This volatility has a negative impact on availability, as a voting machine can become unavailable due to a software or hardware error or attack.

Assurance In paper voting, the lack of trust between participants (officials, voters) is solved by using physical entities, not because these act trustworthily, but because they are inert: they do not act at all. Also, the spatial architecture of the polling station, the voting booth and ballot box determine a certain workflow by their inertness, which provides assurance.

Participating agents (that are not inert) are kept in check due to their limited capacities: most attacks are visible and require equipment and closeness to alter entities such as ballots. Attacking paper voting is akin to violating the assumptions behind it - hiding entities such as ballots violates their visibility property and camera usage violates the inertness property. Security goals are satisfied by providing access control for these entities, by keeping a distance between them and the participants, by observations and limiting the available equipment.

By contrast, completely electronic voting cannot depend on the physical environment for realizing security goals. Ballots can be changed inside the voting machine by the software running inside it. Likewise, the one-way deposit of ballots into the ballot box and the randomization on counting depend on that same software agent. The volatile nature of the software and the votes stored in the machine has a negative impact on assurance. 


\subsubsection{Conflict between Confidentiality and Integrity}

In physical voting, the conflict between confidentiality on the one hand, and integrity and assurance on the other is solved through the limited observation capacities of participants; they can observe enough to detect integrity violations, but not enough to violate confidentiality. Such a mechanisms is not possible for electronic voting: from the perspective of participants many events are invisible, as they occur inside the machine, which gives the software agent control over the outcome of the voting process. Thus electronic voting is unlikely to strike a good balance between realizing the goals of confidentiality and integrity.

\subsection{Hybrid Voting}

We will now investigate four hybrid voting systems and explain the operations and entities used in each of these.

Ballot Printer The ballot printer has a similar user interface as the e-voting machine described earlier, on which the voter can make her choice for a candidate. However, it will print a record of the vote on a paper sheet, and it will not store it in a file.

Optical Scan Machine An optical scan machine consists of a device that scans the ballots and determines the tally, and possible prints it. There are two main ways of operation: the first is that the user scans her own ballot directly after filling in the ballot. The second is that she deposits the ballot in a normal ballot box. After the closure of the election, election officials then insert the ballots into the scanner, potentially with the aid of a device that automatically feeds the ballots to the scanner.

Redundant System There are two types of redundant systems. The first type stores a redundant paper record in addition to the primary electronic procedure. The second type has a redundant electronic process next to the primary paperbased process. Both systems are combinations of a fully electronic system and a vote printer. In the simplest case, the voter will determine her choice on the machine and deposit the print in a separate ballot box.

Scantegrity II The Scantegrity II voting system is a normal paper voting system complemented with extra entities:

- an optical scan machine for the tallying

- a secured computer that generates and stores all the ballot information 


\begin{tabular}{clll}
\hline ID & Threat & Voting system & Goal \\
\hline T19 & Print wrong vote & Vote printer & I \\
T20 & Add additional information on the paper & Vote printer & C \\
T21 & $\begin{array}{l}\text { Introduce inconsistencies between paper } \\
\text { and electronic voting }\end{array}$ & Redundant system & IAS \\
T22 & Change patterns on the paper & Pen system & I \\
\hline
\end{tabular}

Figure 7.14: Threats to voting goals for hybrid voting.

- a printing device for generating ballots

- an online bulletin board where partial results are published

- a special marker pen

The ballots used in Scantegrity II have confirmation codes printed with "invisible ink". Once the user marks a candidate with the special pen, she will see a confirmation code appear on the ballot. She can write this down, together with the ballot ID and check that this result is included in the final tally. (For details we refer to the original paper [29].

Pen System A pen system consists of three parts:

- A pen equipped with a special camera and a cable

- A docking station (connected to the pen) that stores the vote, similar to an electronic voting machine

- Special ballot sheets with small dots in a unique pattern

When the user marks her choice on the ballot, the pen identifies the position on the paper based on the dots that it scanned. This position is then translated to a vote for a particular candidate and stored in the docking station.

\subsubsection{Threats and Mitigations}

Figure 7.14 shows a list of hybrid voting threats, and we explain these further below.

Ballot Printer Ballot printers face similar problems as electronic voting machines: first, they are vulnerable to software and hardware attacks. For example they could alter the vote while printing the ballot (T19), or add additional information on the paper (T20). Second, the emanations can be received by other 
devices. An advantage over electronic voting is that the ballots can be inspected by the user. Compared to normal paper voting, ballot printers can inscribe the ballots more accurate than voters themselves.

Optical Scan Machine Optical scan machines also run software and can thus be made to count the ballots wrongly. Similar to electronic voting, this is hard to detect. However, it is always possible to count the ballots by hand. If the voter scans her own ballot, the optical scan machine can also store additional information such as the time of the vote, thus threatening the confidentiality of the vote.

Redundant System The advantage of redundant systems is that paper and electronic systems have "independent failure modes": if one fails (or is attacked) this does not necessarily threaten the other. This however introduces a new threat: the record must match and discrepancies exist that are not easily solved, casting doubts on the final election outcome (T21). Counting by hand of receipts might be both slow and error-prone [71].

Scantegrity II The threats to Scantegrity II are similar to that of other electronic and optical systems, with additional mitigations that make it more difficult for an attacker to modify the election result, cast doubt on the election amount, or cause a denial-of-service attack. By publishing partial results, voters can be assured that their vote is included in the tally. Data that links the confirmation codes to the final result must be kept secret - if not properly secured, an attacker could steal this information and check the receipts of user, allowing vote buying.

Pen System The pen system also uses software, with the attacks as outlined for e-voting. Furthermore, all ballots must be identical - if the dot patterns are different this would result in different counts (T22).

\subsubsection{Types of Hybrid Systems}

Four out of the five hybrid systems are change digital information to physical form or vice versa.

- The pen system and optical scan machine transform information from physical to digital;

- Scantegrity II and the ballot printer transform information from digital to physical. 


\begin{tabular}{lll}
\hline$\#$ & Explanation & Example \\
\hline S02 & Parallel system & Electronic voting next to digital voting \\
S03 & Serial system & Vote printer \\
\hline
\end{tabular}

Figure 7.15: Types of hybrid systems in the voting case study.

Transformation serves two purposes: first the physical step in each process creates an opportunity for verification because paper is inert. Second the transformation step can improve certain weaknesses in the normal physical process and prevent errors that occur while filling in a ballot, or while counting it. We will call these "serial" systems as physical and digital components are used sequentially in the voting process.

We categorize the remaining redundant system as a "parallelization system" of physical and digital mechanisms as it can perform each step independently.

\subsection{Combinations and Trade-off Analysis}

In this section we compare the hybrid cases to standard paper voting and electronic voting, to assess the benefits of hybrid systems. Figure 7.18 shows a comparison of hybrid systems with completely physical and digital systems. The systems are scored on three goals, confidentiality, integrity and availability.

\subsubsection{Trade-off Analysis on Properties}

Figure 7.16 and 7.17 show the trade-off analysis on the effect of physical and digital properties. Regarding physical properties in some cases negative effects are amplified (active), positive effects are negated (slow, visible) and in yet another case a positive effect is reinforced (difficulty manufacturable). For digital properties most evidence points in the direction that negative effects are reduced and positive effects are reinforced. The only exception is accuracy: hybrid systems are deemed less accurate than their digital counterparts.

\subsubsection{Trade-off Analysis on Goals}

Figure 7.18 shows a direct comparison between hybrid, physical and digital systems on confidentiality, integrity and availability. The results for each system are explained in the next paragraphs.

Ballot Printer Ballot printers improve the integrity of the system compared to paper voting, because they can record the vote more accurately than a person 


\begin{tabular}{lllll}
\hline & & & \\
& & &
\end{tabular}

Figure 7.16: Trade-off analysis on the impact of properties between hybrid and physical for the voting case study. Positive scores indicate that the hybrid system is more secure than the physical system, negative scores that it is not. N/A means that the effect was not observed in the case study. (For the exact operationalization details, see Figure 5.11 in Chapter 5.) The full explanations of the scores for each goal are Section 7.11 


\begin{tabular}{|c|c|c|c|c|c|}
\hline Hypothesis & $\beta^{0}$ & $a^{i}$ & $e^{2}$ & $\mathrm{~s}^{\mathrm{C}^{0}}$ & $\mathbf{p}^{e^{\prime}}$ \\
\hline $\begin{array}{l}\text { Accurate Data Storage, } \\
\text { Transportation and } \\
\text { Processing CIA+ }\end{array}$ & $=/-$ & $=/-$ & $=$ & $=/-$ & $=$ \\
\hline $\begin{array}{l}\text { Accurate Data Storage, } \\
\text { Transportation and } \\
\text { Processing I- }\end{array}$ & + & + & + & + & + \\
\hline Fast Data Processing A- & + & + & $=/+$ & + & $=/+$ \\
\hline Fast Data Storage C- & $=$ & + & $=$ & $=$ & $=$ \\
\hline $\begin{array}{l}\text { Program Computational } \\
\text { Complexity CIA- }\end{array}$ & $=/+$ & $=/+$ & $=/+$ & $=/+$ & $=/+$ \\
\hline
\end{tabular}

Figure 7.17: Trade-off analysis on the impact of properties between hybrid and digital for the voting case study. Positive scores indicate that the hybrid system is more secure than the digital system, negative scores that it is not. N/A means that the effect was not observed in the case study. (For the exact operationalization details, see Figure 5.11 in Chapter[5) The full explanations of the scores for each goal are Section 7.11.

\begin{tabular}{|c|c|c|c|c|c|c|}
\hline & & $\boldsymbol{B}^{\mathbf{1}}$ & $0^{x}$ & $\mathrm{re}^{\mathrm{e}^{v^{3}}}$ & & $e^{5^{55^{5}}}$ \\
\hline \multirow[t]{2}{*}{ Confidentiality } & Physical & -- & - & -- & - & -- \\
\hline & Digital & \pm & ++ & $=/-$ & + & \pm \\
\hline \multirow[t]{2}{*}{ Integrity } & Physical & + & + & + & + & + \\
\hline & Digital & + & + & + & + & + \\
\hline \multirow[t]{2}{*}{ Availability } & Physical & - & \pm & - & - & - \\
\hline & Digital & + & + & $=/-$ & + & $=/+$ \\
\hline
\end{tabular}

Figure 7.18: Comparison of hybrid voting systems with physical and electronic systems. Each column compares a hybrid system. For example a ballot printer is compared to the pure physical or electronic system discussed before. (For the exact operationalization details, see Figure 5.11 in Chapter[5.) The explanation of the scores for each goal is given in Section 7.11 
could. Compared to electronic voting, the advantage of a ballot printer is that the ballot can be visually inspected and is more difficult to change.

Because ballot printers are electronic, their software is vulnerable to attacks. As the ballot printer "knows" the vote it is threatening to the confidentiality. It can either record the vote internally or print hidden information on the ballot that can be used later to identify the voter. Because of this possibility, ensuring confidentiality with a ballot printer is very difficult. One advantage compared to electronic voting is that the ballot printer delivers a tangible result that can be deposited.

Regarding availability, a ballot printer is less reliable than a paper voting system, because the hardware and the software can always break down. Compared to a digital system, a ballot printer can however be more easily replaced in case of failures, and hardware failure also does not hinder the storage of the votes.

Optical Scan Machine Regarding integrity, the advantage of an optical scan machine over a physical process is that it has the potential to count votes more accurate and allow for quick recounts as well. Additionally, the scans can be stored for analysis to check the integrity of the process. Compared to electronic voting, the advantage is that the counting process can be verified by a person.

As for availability, the same arguments hold as for the ballot printer: first an optical scan machine can break down, is this less reliable than a physical process. Second, it can be replaced easier than an electronic voting system.

Also similar to the ballot printer, the software of the optical scan machine is vulnerable to attacks and can be used to produce a wrong count or to identify voters, either because the ballots are scanned directly after the person fills in the ballot, or afterwards [24]. This has a negative impact on confidentiality. However, an optical scan machine can offer more guarantees for the confidentiality of the data compared to an electronic voting machine, because there does not have to be a direct connection (in time and space) between the machine and the voter.

Redundant System A parallel redundant digital and physical system inherits all the confidentiality problems of a digital system and scores thus negative compare to a pure physical system. Compared to a digital system, a redundant system can have the additional problem that it threatens confidentiality if paper records are not randomized (in case of a continuous audit trail). Integrity can be improved over both paper and e-voting, because the vote is stored in multiple ways, which makes manipulation more difficult. Again because of failures in hardware and software, a redundant system (that requires both the physical system and the digital system to be active) scores negative on availability compared to paper voting. Availability would be equal or less than a digital system, because of possible failures in the paper system. 
Scantegrity II As each ballot is unique and the user receives a receipt, there is a bigger potential threat of confidentiality loss compared to a physical system, but because the carrier is physical, it scores better than a digital system.

The integrity of the vote is aided by the invisible ink system described earlier, which is a clear improvement over paper voting. Again, because the vote is stored on paper, the integrity is also better compared to electronic voting.

Also hardware and software failures have a negative impact on the availability of Scantegrity II compared to a physical system, but because a physical carrier is used, the availability is high than that of a pure electronic system.

Pen System Regarding confidentiality, similar arguments hold for the ballot printer and the pen system: they both capture the vote electronically and threaten confidentiality, whereas the advantages over a pure digital system are less clear. Integrity is improved over both electronic and paper voting, because as the vote is stored electronically and on paper. Hardware and software faults make the pen system less available as a paper system. Because of the simplicity of the system it is likely better than that of a purely digital system.

\subsection{Conclusion}

\subsubsection{Properties}

Paper voting mainly works through two properties: first, persons have limited visibility of all entities: they can observe enough to be assured of the integrity of the process, but not as much to threaten the confidentiality. Inertness also has a positive effect on assurance. There is a lack of trust between all parties (and thus of trusted third parties) However all participants trust the polling station, and its contents, such as ballot boxes and ballots to be inert, not under active control by another participant. Thus, the polling station functions as a neutral terrain, a "trusted third space", which solves the trust problem. Digital technology such as smartphones with cameras violate the inertness property of the voting booth, allowing a recording of the vote.

\subsubsection{Comparison}

In the absence of high-tech equipment such as cameras and sensors, the physical paper voting process can ensure adequate confidentiality and integrity. A direct comparison between completely electronic voting and paper voting leads to the conclusion that electronic voting scores less on all security goals. 


\subsubsection{Types of Combinations}

Two types of combinations were discovered in this chapter: transformation systems, which transform information from digital to physical form (or vice versa) or parallelization systems that execute a physical and a digital process simultaneously.

\subsubsection{Trade-off Analysis}

For each piece of hardware or software added to a paper voting process, doubts increase about the confidentiality of the vote because each component can potentially record the voting process. It is impossible to guarantee that these do not violate vote secrecy or improve the ability of other actors to do so. However adding hardware and software can improve the integrity of the vote. This is true for optical scanners, ballot printers and pens.

\subsubsection{Possibilities for Electronic Voting}

We will now answer question $\mathbf{R Q 5} \mathbf{5}_{\text {Voting }}$ about the possibilities for electronic voting. A completely digital voting system that is designed to maximally satisfy the confidentiality requirement (meaning, it does not offer any evidence to the voter that her vote is counted) is flawed: its integrity is not beyond doubt, and the lack of integrity paradoxically casts doubts about the confidentiality. Adding more digital components decreases confidentiality. However, up to a certain point, they can improve integrity because the digital components can compensate for errors in the physical process (for example marking a ballot or counting the votes). This is quantitatively illustrated in Figure 7.19 .

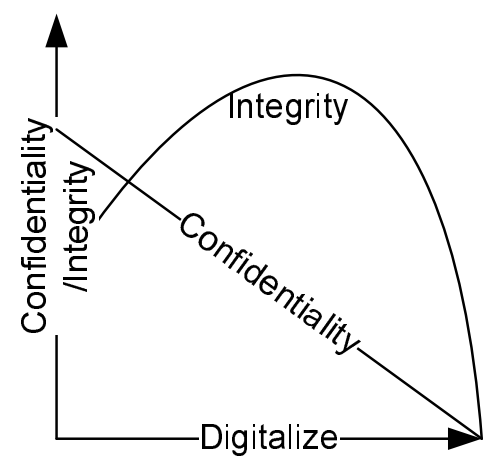

Figure 7.19: Trade-off between confidentiality and integrity for voting systems. As a voting system becomes more digital, the confidentiality, decreases. Up to a point, the integrity is increased but then drops as the system becomes fully electronic. 


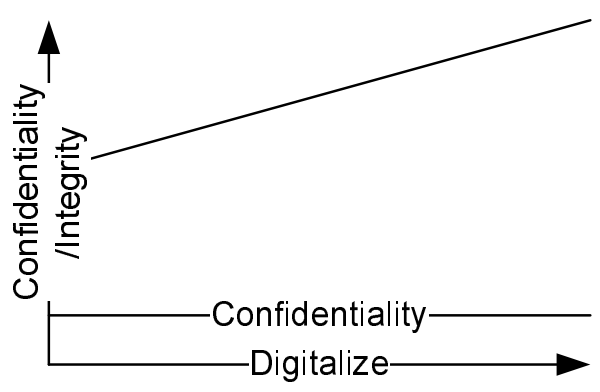

Figure 7.20: Effect of automation on integrity, if the confidentiality requirement is dropped. As a voting system becomes more digital, the integrity steadily increases.

However, another trade-off is possible, namely to drop the confidentiality requirement of the design of the voting system a-priori, and aim for a higher assurance of the integrity of the vote 5 Adding more digital systems then improves integrity, and a completely digital system can assure almost complete integrity. Such a system is conceptually simple: we can record the procedures of the polling station, have the vote take place in public and publish all results on the Internet for everyone to verify. This trade-off is illustrated in Figure 7.20 The more components are automated, the more the system's integrity increases, as it becomes easier to verify the results.

${ }^{5} \mathrm{Cf}$. the term software independence as proposed by Rivest [178]: "An undetected change error in software should not cause an undetected change in election outcome." This term is about assurance of the integrity of the result, and not about confidentiality. Rivest considers software independence to be a good thing, as it allows independent verification. 


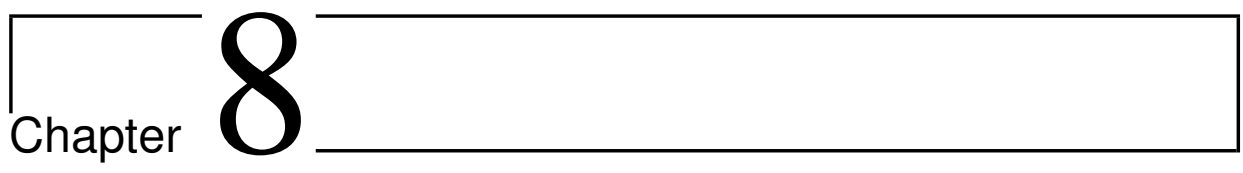

\section{IT Infrastructure}

\subsection{Introduction}

In this chapter 1 we will investigate physical and digital security in the context of IT infrastructure. IT infrastructure is operationalized here as the hardware and software on which business applications are run 2 Physical security used to play an important role in IT infrastructure security: engineers had to be physically present in the data center to perform certain tasks, and applications running on one physical machine could not be easily transferred to other machines. However virtualization (the construction of a system using software from underlying physical resources, see Chapter 2 has become a key technology in today's data centers. It turned once dedicated physical computing resources such as servers into digital resources that can be provisioned on demand without the need of a human presence inside the data center. Virtualization is also one of the enabling technologies of cloud computing. As in all our case studies, we investigate security characteristics of physical, digital and hybrid systems.

We define physical infrastructures as those systems that do not have a virtualization layer. Virtualized infrastructures are those systems that are completely virtualized and hybrid infrastructures are only partially virtualized.

Our main motivation for researching the virtualization of IT infrastructures is that in spite of its widespread usage, the security impact of virtualization is not completely clear. The opinions in the scientific community on virtualization are diverse, as the technology contains both strengths and weaknesses. Claimed security improvements of virtualization are the increased availability of applications

\footnotetext{
${ }^{1}$ This chapter is based on Van Cleeff et al. [210] and Van Cleeff et al. [208].

${ }^{2}$ COBIT defines IT infrastructure as [sic] the technology and facilities (hardware, operating systems, database management systems, networking, multimedia, and the environment that houses and supports them) that enable the processing of the applications [96].
} 
and the isolation of processes [22]. Virtualization also has security drawbacks, such as exploitable weaknesses in virtualization software, the existence of covert channels and the possibility of new types of malware [52]. Researchers are also investigating means to improve cloud security in the data center itself through specific hardware solutions [16]. The deficiencies of logical authentication services, such as password reset via email, have also led cloud vendors to provide their users with hardware tokens [3]. VMM vendors such as VMware have written extensively on virtualization security, guiding enterprises on how to perform infrastructure hardening [216] and server configuration [219]. General advice is that virtual machines should be secured as if they were physical machines, and be equipped with intrusion detection systems and anti-virus.

Section 8.2 contains a short introduction to virtualization and cloud security. Next Section 8.3 explains our data collection methods. In Section 8.4 the goals are presented. Section 8.5 starts the investigation of the infrastructures with an examination of physical systems. Next the properties of physical entities are derived in Section 8.6, answering RQ1. We continue with virtualized infrastructures (Section 8.7) and the investigation of digital properties in Section 8.8 (RQ2), after which we compare physical and hybrid systems (RQ3) in Section 8.9 . Section 8.10 analyzes types of combinations of virtual and nonvirtual systems (RQ4). Finally we perform a trade-off analysis in Section 8.11 (RQ5). A specific question we intend to answer is $\left(\mathbf{R Q} 5_{\text {infra }}\right)$ : are there limits to what can be securely virtualized? Given an existing data center, we would like to understand whether is it possible to virtualize all servers and yet maintain an adequate level of security. This issue is especially relevant as virtualization is one of the enabling technologies of cloud computing, which tends to replace physical with digital security controls on an unprecedented scale.

\subsection{Conceptual Framework}

\subsubsection{Definition and Concepts}

We will start with explaining the concept of virtualization, which we define as a software layer that implements a physical architecture [210]. The layer exposes an interface to other systems, effectively decoupling these systems from the hardware. The systems running on top of the virtualization layer depend less on hardware specific implementations, and virtualization can therefore improve portability, resource sharing and management.

We can categorize virtualization by the state of the data that is affected: data in transit (network virtualization), data at rest (storage virtualization) and data in processing (server virtualization). Network virtualization is possibly the most common type of virtualization: instead of placing individual cables between servers, cables are connected to central switches, where digital separations are made be- 
tween network sections. The second type of virtualization is storage virtualization. Each physical server no longer stores data on its individual hard disks but in a centrally managed storage array network (SAN), of which each server receives a part. Finally with server (or machine) virtualization, operating systems are presented with a virtual CPU and memory 3

Regarding the management and control of virtualized infrastructures, the software layer exposing this CPU is called the virtual machine monitor (VMM) or hypervisor. The VMM allows the physical server to be shared between multiple operating systems at the same time. Multiple physical servers equipped with VMMs can be bundled together to form virtualization clusters, and virtual machines can migrate between these servers, to optimize server load and maintenance schedules. Currently, this layer does not have a widely accepted name (the term "cloud OS" is a potential candidate). To emphasize that this layer controls the VMMs, we call this infrastructure and its management the "VMMM", an acronym for virtual machine monitors' management.

\subsubsection{Summary and Implications for Case Study}

The investigation of virtualization technologies resulted in a typology of types of virtualization (network, storage and processing). Figure 8.1 shows these types together with the corresponding physical and digital entities.

\begin{tabular}{lll}
\hline Virtualization Type & Original Physical Entity & Virtualized Entity \\
\hline Storage Virtualization & Hard disk & SAN \\
Network Virtualization & Cross-cable & Switch \\
Machine Virtualization & Rack server & VMM/Hypervisor \\
\hline
\end{tabular}

Figure 8.1: Virtualization types and corresponding physical and virtualized entities.

A picture of the different virtualization management levels (VM, VMM and VMMM) is shown in Figure 8.2. It shows virtualization-capable hardware, several VMs run on top of a group of VMMs and VMMs being managed by a VMMM. We will use this typology to assess the threats of virtualization in Section 8.7.1

\subsection{Method}

Our study of IT infrastructures diverts from the approach in the other case studies, as there is no specific physical, digital or hybrid system to be studied.

\footnotetext{
${ }^{3}$ For a taxonomy of server virtualization we refer to Smith and Nair [197].
} 


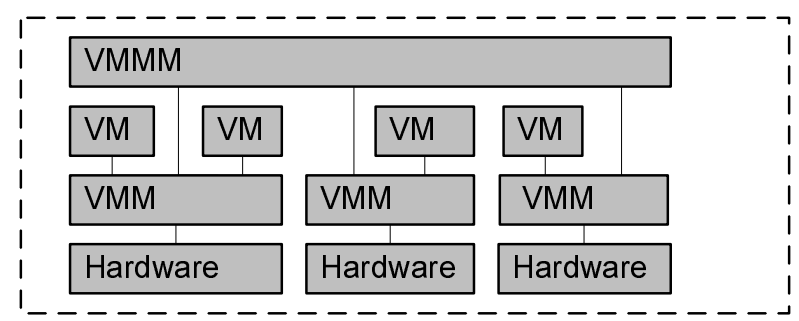

Figure 8.2: Virtualization entities.

Instead we did a literature study on virtualization and then performed case studies at three organizations that each used hybrid IT infrastructures, meaning that they were partially virtualized. The focus of the chapter is thus on virtualization security and less on physical system security.

\subsubsection{Case Study Selection and Method}

In this case study, we collected data through two means. First a literature study was done on what is known about virtualization. Second we performed interviews at several organizations that were using virtualization. Figure 8.3 explains which data source is used for which subsequent section.

\begin{tabular}{|c|c|}
\hline Section & Source \\
\hline 8.4 Goals & Literature study \\
\hline 8.5 Physical IT Infrastructure & Interviews \\
\hline 8.6 Properties of Physical Entities & Derived from 8.5 \\
\hline $\begin{array}{l}8.7 \text { Virtualized IT Infrastructure - opera- } \\
\text { tions }\end{array}$ & Interviews \\
\hline $\begin{array}{l}\text { 8.7 Virtualized IT Infrastructure - threat } \\
\text { model }\end{array}$ & Literature study \\
\hline $\begin{array}{l}8.9 \text { Comparison between Physical and Vir- } \\
\text { tualized Infrastructure }\end{array}$ & $\begin{array}{l}\text { Derived from previous sec- } \\
\text { tions and interviews }\end{array}$ \\
\hline 8.10 Hybrid Infrastructure & Interviews \\
\hline 8.11 Combinations and Trade-off Analysis & $\begin{array}{l}\text { Derived from previous sec- } \\
\text { tions }\end{array}$ \\
\hline
\end{tabular}

Figure 8.3: Data sources for sections. 


\section{Literature Study}

For this study, we began with a literature search on Scopus 4 , using keywords "virtualization" and "security". This yielded a total of 151 papers of which 46 were relevant. Literature from other sources was also examined, including datasheets from virtualization product vendors such as VMware. We chose to present the results centered around specific features of virtualization. Obviously, a complex technology such as virtualization can be split up in many ways, and each choice is to some extent arbitrary. For this model, we used the following guidelines:

1. A feature represents a distinct piece of functionality, characteristic or architecture, having a unique impact on security.

2. Existing decompositions found in the literature should be used as much as possible.

3. A feature should be widely used, not just being implemented by one vendor in one product.

For each feature, we present the security effects, together with a key reference. When no direct security effects were found, we added analytical claims based on the literature studied. Apart from clearly identifiable features of virtualization, we also examine features arising from unintended interactions between features. We will call these "emergent features".

\section{Case Studies}

Aided by the results of the literature study, we then performed three case studies of private cloud 5 that use virtualization technology. These clouds employ a mixture of physical and digital security mechanisms and we investigate the security impact of replacing the former with the latter as well as possible combinations.

Sampling and Data Collection From 2009 to 2010 we performed case studies at three organizations. These are:

- $\mathrm{X}$, an organization servicing over 5000 internal and external customers;

- Y, a financial service provider with over 100 million Euros in assets;

- Z, a payment service provider.

\footnotetext{
4 http: //www. scopus. com

${ }^{5}$ A private cloud is a cloud specially dedicated for one organization.
} 
These organizations were selected based on expressed interest in participation and accessibility, and range from having a very limited interest in security $(\mathrm{X})$ to being extremely concerned about security (Z). (See section 8.3.2 for a discussion of the validity of the results.)

We developed a case study protocol with detailed questions, and we performed in-depth interviews with administrators and risk managers, and obtained documentation concerning the architecture and management of the organizations' data centers. Research was done under NDA, after which participants received draft publications, checked for errors and approved the publication 6 Typically we met with our case study partner once, except for case $X$ where three interviews were done (Figure 8.4).

Regarding case study partner $X$, interviews were held on-site on the 11th, 18th and 26th of May 2010. During the first interview, we discussed the design of the virtualization infrastructure. In the second meeting, we zoomed in on the physical boundaries of the data centers, and in the last meeting we explored the organization's virtualized file storage system.

As for case study partner $Y$, an interview was organized through the company's IT security auditor. This took place on October 8th, 2010. Prior to this, we received documents about the audit results, which were discussed during the interview. Given the level of detail in the report, the interview focused on issues that were out of scope for the audit.

The interview with participant $Z$ was the most broad, and focused on the differences between physical and digital security. It took also place on site, at June 26th, 2009. In a session of about two hours, four questions were discussed with the participants:

Q1 : Which procedures for management of hardware and software have digital and physical equivalents?

Q2 : For each type of procedure, which differences are there between the physical and the digital variants?

Q3 : From these differences, what underlying physical and digital properties can we distill?

Q4 : Knowing these differences, how should we design systems and procedures for optimal security?

Figure 8.5 shows which types of data were obtained for each case. The amount of data that could be collected varied from case to case, depending on time con-

\footnotetext{
${ }^{6}$ The consequence of the NDA's is that it is not possible here to add potentially interesting information that was left out of the earlier publications, because this would have to be approved once more prior to publication of this thesis.
} 
straints of IT staff, third parties involved, and concerns over the security consequences of the research itself.

\begin{tabular}{lccc}
\hline Interview & $\mathbf{X}$ & $\mathbf{Y}$ & $\mathbf{Z}$ \\
\hline Interviews with administrators & $3 \mathrm{x}$ & $1 \mathrm{x}$ & $1 \mathrm{x}$ \\
Interview with IT risk managers & - & - & $1 \mathrm{x}$ \\
\hline
\end{tabular}

Figure 8.4: Interviews for case studies.

\begin{tabular}{lccc}
\hline Data Obtained & X & Y & $\mathbf{Z}$ \\
\hline Network architectures & D & D & I \\
List of physical servers & D & D & I \\
Data center layout & D & D & I \\
Security audit results & - & D & - \\
Installation procedures for servers & D & D & I \\
\hline
\end{tabular}

Figure 8.5: Data collected from case studies. $\mathrm{D}=$ primary documents, $\mathrm{I}=$ interview.

Data Analysis In Chapter 2 we already stated that digital processes always have a physical basis. For our study of IT infrastructures this means that when we investigate a virtualized system it will already have been installed on hardware. However, for digital procedures, hardware installation is a one-time event, after which everything can be done digitally, without modifying the hardware. Thus, in effect, we compare physical procedures with digital procedures after hardware has been installed.

\subsubsection{Limitations and Scope}

Virtualization can be used for many purposes, for example software testing or providing uniform desktops to end-users (desktop virtualization). Obviously, the security effects differ for each type of usage. We concentrate on the security effects for running production applications, where a business's applications are placed inside VMs. The reason for this choice is that compared to other usage scenarios, business applications are more business critical with correspondingly high security requirements.

Validity Concerning the case studies, we guarded the internal validity of our study by sending our initial results to each participant and made several corrections or additions hereafter. 
Regarding the external validity, our sampling method was theoretical, meaning that we selected heterogeneous cases which each could highlight different aspects of the digital-physical trade-off in different organizational settings. We especially took effort to include an organization that was extremely security-conscious, because we anticipated that important security problems of virtualization might only be noticeable in such a situation. With a theoretical sampling method, results are generalizable, not because of statistical sampling techniques, but because the mechanisms identified in these case studies are generalizable for the entire population of cases. Translated to our study, this means for example that the entities and operations are present in any organization that uses virtualization technology. Regarding the systems studied, all our participants used variants of VMware's ESX, which is the market leader for virtualization software.

\subsection{Goals}

We now begin to construct the KAOS tree, starting with the goals. For IT infrastructures, COBIT [96] provides a framework for managing the IT processes that realize these properties. In a top-down approach, an enterprise must state its business and IT goals, the processes that contribute to these goals, and the control objectives for these processes. Controls are the business processes (policies, procedures, practices) that provide assurance that business objectives are achieved and if not, that undesired events are prevented and/or detected.

In this case study, we will use COBIT as a source of relevant security objectives or goals. Similar frameworks include ITIL and ISO 27002:2005, and mappings exist between goals listed in these three frameworks [97]. Thus, by using COBIT, the results are also linked to these frameworks. What is important is not the exact chosen framework, but rather that through this link, the relevance of the results presented in this chapter is more obvious for enterprises, as they have to implement these control objectives. As for the choice of controls, there are many more options (for example, ISO 27002 has 133 security controls). In this case study, we focus on controls directly relevant for IT infrastructure management, and especially those for which it can be expected that there are major differences between physical, digital and hybrid systems.

Of the available control objectives defined in COBIT, we selected six control objectives that were relevant to security. These goals are further explained in Figure 8.6 For easier understanding, we place the goals into the six categories and use these throughout this chapter, instead of the COBIT names.

Figure 8.7 shows all the goals that will specifically used in this case study, independent of their digital, physical or hybrid realization. Thus, we chose "support business objectives" (G1) as the top goal for this IT infrastructure case study. This goal has two subgoals. 


\begin{tabular}{|c|c|c|}
\hline Goal & Explanation & $\begin{array}{l}\text { COBIT Control } \\
\text { Objectives }\end{array}$ \\
\hline Redundancy (G11) & $\begin{array}{l}\text { Ensure that there is no } \\
\text { single point of failure. }\end{array}$ & $\begin{array}{l}\text { DS4 Ensure Continuous } \\
\text { Service }\end{array}$ \\
\hline Partitioning (G9) & $\begin{array}{l}\text { Separate systems to limit } \\
\text { propagation of undesired } \\
\text { events. }\end{array}$ & DS5.10 Network Security \\
\hline Access control (G13) & $\begin{array}{l}\text { Ensure that only authorized } \\
\text { personnel has access. }\end{array}$ & $\begin{array}{l}\text { DS5.3 Identity } \\
\text { Management, } \\
\text { DS5.4 User Account } \\
\text { Management }\end{array}$ \\
\hline Work instructions (G10) & $\begin{array}{l}\text { Provide detailed and precise } \\
\text { instructions so that changes } \\
\text { are executed correctly. }\end{array}$ & DS5.2 IT Security Plan \\
\hline Monitoring (G12) & $\begin{array}{l}\text { Log system events and } \\
\text { check for undesired events. }\end{array}$ & $\begin{array}{l}\text { DS5.5 Security Testing, } \\
\text { Surveillance and } \\
\text { Monitoring }\end{array}$ \\
\hline Implementation (G3) & $\begin{array}{l}\text { Actually build the IT } \\
\text { systems. }\end{array}$ & $\begin{array}{l}\text { AI7 Install and Accredit } \\
\text { Solutions and Changes }\end{array}$ \\
\hline
\end{tabular}

Figure 8.6: Control categories in relation to COBIT definitions.

The first sub-goal is security (G2): we examine whether systems can realize the common security goals of confidentiality, integrity, availability (G4, G5, G6). We add to this also the goals of non-repudiation (G7) and authenticity (G8) from Chapter 2. These are important security goals, and the case study participants and our literature study indicated that virtualized systems have difficulty in achieving them; by listing them individually it becomes easier to highlight these challenges.

The second sub-goal is the actual installation of IT resources (G3): without any implementation it is impossible to support any business process. (This situation is similar to the voting case study from Chapter 7 where we added the the goal that eligible voters actually vote in the election.)

As mentioned earlier in Chapter 2, the standard security goals of confidentiality and availability conflict: ensuring confidentiality of data implies limiting the number of copies in circulation, as even one stolen copy is sufficient to cause a confidentiality breach. By contrast, availability requires more copies (and more system connectivity). This is visible in the goal tree, where the goals of DS4 (Redundancy or Continuous Service) and DS5.10 (Partitioning or Network Security) conflict. In the next sections we examine how different types of systems the aforementioned goals and in how far they can solve this goal conflict. 


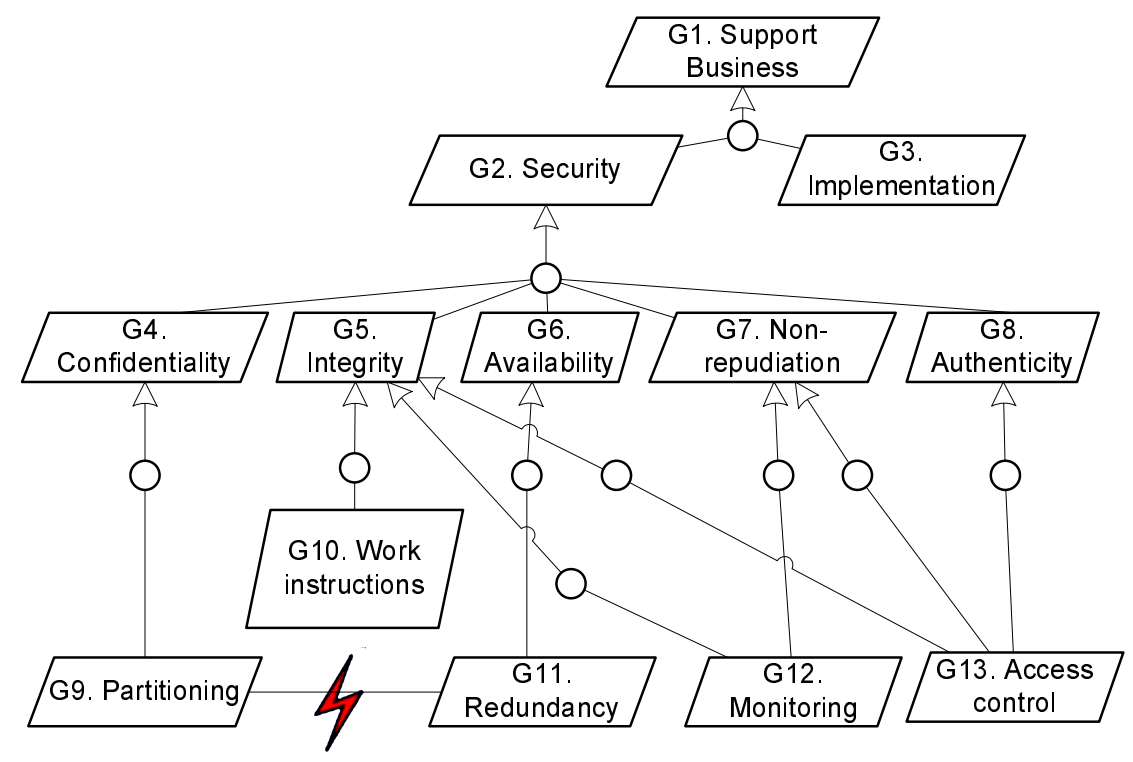

Figure 8.7: IT Infrastructure goal tree.

\subsection{Physical IT Infrastructure}

\begin{tabular}{llllrl}
\hline ID & Name & ID & Name & ID & Name \\
\hline E1 & Hard disk & E5 & Engineer & E9 & Keys \\
E2 & (Cross-)cable & E6 & Switch & E10 & Data center \\
E3 & Rack server & E7 & Server cabinets & & \\
E4 & Surveillance cameras & E8 & Locks & & \\
\hline
\end{tabular}

Figure 8.8: Selected entities used in physical IT Infrastructures.

Having presented the technology independent goals for IT infrastructure, we now begin the specific physical part on infrastructures with a list of entities, as shown in Figure 8.8. (Obviously this list can be extend with other entities, such as doors and rooms, in this case study we focus primarily on entities that did not already occur in the voting case study of Chapter 7.) We will now discuss the operations (or procedures) that can be executed on physical systems. Figure 8.9 lists procedures found through one or more of our interviews and relates them to the goals from Section 8.4. (Again, this list can be extended to include more procedures, we focus here on those procedures that were discussed during the interviews.) 


\begin{tabular}{lllll}
\hline ID & Operation & Goal & Agent & Entities \\
\hline P1 & $\begin{array}{l}\text { Connect/disconnect } \\
\text { switches and servers }\end{array}$ & Implementation & Engineer & $\begin{array}{l}\text { Cable, switch, } \\
\text { server }\end{array}$ \\
P2 & Add/remove disks & Implementation & Engineer & Hard disk \\
P3 & $\begin{array}{l}\text { Add/remove CPUs, } \\
\text { memory, motherboards }\end{array}$ & Implementation & Engineer & Server \\
P4 & Install software & Implementation & Engineer & Server \\
P5 & Surveillance of persons & Monitoring & Engineer & Surveillance \\
& & Monitoring & Engineer & Server, \\
P6 & Visual inspections & Switch, cable \\
& & Monitoring & Engineer & \\
P7 & Accompany visitors & & & \\
\hline
\end{tabular}

Figure 8.9: Physical operations and their contributions to goals.

The first three $(P 1, P 2$ and $P 3)$ concern infrastructure implementation at the network, storage and processing levels. The fourth operation ( $P 4$ is application management, the installation and configuration of applications. This done directly onto the server, using monitor, keyboard and mouse. Also listed are specific operations for the goal of monitoring (P5,P6 and P7).

We now briefly consider threats against physical infrastructure, based on interviews with our case study participants. Our analysis yields three types of threats shown in Figure 8.10. A major threat is that the wrong component are connected or disconnected (T1), either maliciously or by accident. Sometimes inspections can detect these errors, but these can also fail (T3). Finally during the changes themselves direct oversight of the engineers can fail as well (T2).

\subsection{Properties of Physical Entities}

We will now investigate the security properties of physical security mechanisms (RQ1). This is done based on the data from the previous section, about the roles that entities and procedures play in realizing security goals. This is done through the method discussed in Chapter 5. Figure 8.11 shows the updates to the

\begin{tabular}{cll}
\hline ID & Threat & Threat to Goal \\
\hline T1 & Connect/disconnect wrong components & Redundancy, partitioning, access \\
& & control \\
T2 & Do not monitor engineers & Monitoring \\
T3 & Do not detect configuration errors & Monitoring \\
\hline
\end{tabular}

Figure 8.10: Threats to goals for physical IT infrastructure. 
existing hypotheses from the earlier case study. Most earlier hypothesis are supported and additional conditions have been added. Next, Figure 8.12 shows the list of newly discovered properties and their impacts. In the next paragraphs, each property is described in more detail.

\begin{tabular}{|c|c|}
\hline Property and Effect & Details \\
\hline $\begin{array}{l}\text { Active CIA- } \\
\text { Active CIA+ } \\
\text { Decaying IA- } \\
\text { Dense CIA+ } \\
\text { Difficultly } \\
\text { manufacturable CI+ }\end{array}$ & $\begin{array}{l}\text { Attacker connects the wrong servers. } \\
\text { Person installs cables. } \\
\text { Not observed. } \\
\text { Data center has strong physical protection. } \\
\text { Manufacturing servers is complicated. } \\
\text { Counterexample: Cables are easy to obtain but cannot easily } \\
\text { impact the business process unless not observed. } \\
\text { Conditions: Business process depends on limited and/or } \\
\text { unique items. }\end{array}$ \\
\hline $\begin{array}{l}\text { Easily } \\
\text { manufacturable CI- } \\
\text { Enclosed with } \\
\text { opening C+ }\end{array}$ & $\begin{array}{l}\text { Not observed: unique objects are hard to manufacture and } \\
\text { simpler objects such as cables are not expected to be unique. } \\
\text { Actions inside the data center are confidential. }\end{array}$ \\
\hline Inert CIA+ & Cables cannot change place by themselves. \\
\hline Invisible $\mathrm{C}+$ & Confidential contents of a SAN is not visible. \\
\hline Large I+ & Cables are too large to be smuggled. \\
\hline $\begin{array}{l}\text { Limited spacious } \\
\text { CIA+ }\end{array}$ & $\begin{array}{l}\text { Rack space is limited and therefore it is difficult to hide a } \\
\text { server without being detected. }\end{array}$ \\
\hline Modifiable CI- & $\begin{array}{l}\text { Not observed. } \\
\text { Counterexample: An attacker can modify objects but the } \\
\text { effect is damage, not a threat to confidentiality or integrity. } \\
\text { Conditions: The attacker precisely modifies objects. }\end{array}$ \\
\hline Modifiable CI+ & $\begin{array}{l}\text { Not observed (objects are not modified, only moved, } \\
\text { connected and disconnected.) }\end{array}$ \\
\hline Movable CIA- & Attacker connects a cable incorrectly. \\
\hline Movable CIA+ & Person moves a cable. \\
\hline Slow CIA+ & $\begin{array}{l}\text { An attacker must travel to the data center. } \\
\text { Counterexample: Attacker is already present, for example as } \\
\text { an insider. } \\
\text { Conditions: Attacker is already present. }\end{array}$ \\
\hline Strong CIA- & $\begin{array}{l}\text { An attacker damages the physical server infrastructure. } \\
\text { Counterexample: Protection relies mainly on strong } \\
\text { physical protection and monitoring. } \\
\text { Conditions: The security mechanism is physically weak. }\end{array}$ \\
\hline Strong CIA+ & $\begin{array}{l}\text { Data center walls are difficult to destroy. } \\
\text { Counterexample: Secrets cannot be read easily from a } \\
\text { Storage Array Network (SAN). } \\
\text { Conditions: The attacker does not have the capability to } \\
\text { retrieve the confidential information. }\end{array}$ \\
\hline Visible C- & Not observed (no confidential information is displayed). \\
\hline
\end{tabular}




\begin{tabular}{ll}
\hline Property and Effect & Details \\
\hline Visible CIA- & An attacker waits until she is alone. \\
Visible CIA+ & $\begin{array}{l}\text { Guards detect changes in arrangement of hardware and } \\
\text { observe the actions of malicious engineers. } \\
\text { Counterexample: Cables are hard to trace so that the impact } \\
\text { of actions is not always clear, causing a negative impact on } \\
\text { security. } \\
\text { Conditions: Defenders understand the security effects of } \\
\text { (attacker) actions. } \\
\text { Attacker cannot easily intrude into a data center. }\end{array}$ \\
\hline
\end{tabular}

Figure 8.11: Support for existing hypotheses (consisting of properties and their effects) in the IT infrastructure case study. If counterexamples lead to new hypotheses they are listed individually in Figure 8.12

Cohesive Object cannot be used to hide other objects. An attacker cannot smuggle things easily and this has a positive effect on information security. An example specific to this case is that it is difficult to hide a cable.

Identifiable A defender must understand the physical status of the process that depends on the presence or absence of specific items and detect items that are necessary (and not there) or forbidden (but are present) or changed (not allowed). Thus entities must be identifiable, which positively impacts security. An example specific to this case is that items in a server room should be identifiable.

\begin{tabular}{lll}
\hline Property & Effect & Hypothesis \\
\hline Cohesive & CIA+ & $\begin{array}{l}\text { An attacker cannot use a cohesive object to hide other } \\
\text { objects inside it. } \\
\text { Identifiable }\end{array}$ \\
CIA+ & $\begin{array}{l}\text { A defender identifies objects to understand the security } \\
\text { status of the process. }\end{array}$ \\
\hline
\end{tabular}

Figure 8.12: New hypotheses from the IT infrastructure case study about the security effects of physical properties of entities. Each row represents either positive or negative effects. Multiple letters indicate an effect on multiple security goals. For example CIA- means a negative effect on confidentiality, integrity and availability. 


\subsection{Virtualized IT Infrastructure}

Based on our analysis in Section 8.2, we identify six new entities for virtualization. shown in Figure 8.13 .

\begin{tabular}{lll}
\hline ID & Entity & Virtualization Type \\
\hline E1 & SAN & Storage \\
E2 & Switch & Network \\
E3 & Virtualization-capable hardware & Machine \\
E4 & Virtual machines (VM) & Machine \\
E5 & Virtual machine monitor (VMM) & Machine \\
E6 & Virtual machine monitor management layer (VMMM) & Machine \\
\hline
\end{tabular}

Figure 8.13: Overview of virtualization entities.

We will now discuss the operations (or procedures) that can be executed on virtualized systems. In Figure 8.14, we list procedures found in one or more of our case studies. (Contrary to other cases, no further explanation is given, as we assume these operations to be self-explanatory.)

\begin{tabular}{|c|c|c|c|c|}
\hline ID & Operation & Agent & Goal & Entities \\
\hline $\mathrm{P} 1$ & $\begin{array}{l}\text { Configure switches, VMM, } \\
\text { operating system, firewall }\end{array}$ & Engineer & Implementation & $\begin{array}{l}\text { VMM, } \\
\text { switch }\end{array}$ \\
\hline $\mathrm{P} 2$ & $\begin{array}{l}\text { Configure SAN, VMM, operating } \\
\text { system }\end{array}$ & Engineer & Implementation & $\begin{array}{l}\text { VMM, } \\
\text { SAN }\end{array}$ \\
\hline P3 & Configure VMM, operating system & Engineer & Implementation & VMM \\
\hline $\mathrm{P} 4$ & $\begin{array}{l}\text { Install from remote console or } \\
\text { VMM }\end{array}$ & Engineer & Implementation & VMM \\
\hline P5 & $\begin{array}{l}\text { Create snapshots, dynamically } \\
\text { migrate machines }\end{array}$ & Engineer & Redundancy & $\begin{array}{l}\text { VMM, } \\
\text { VM }\end{array}$ \\
\hline P6 & Configure firewalls and switches & Engineer & Partitioning & VMM \\
\hline P7 & Limit snapshots and replication & Engineer & Partitioning & VMMM \\
\hline P8 & $\begin{array}{l}\text { Implement separation of duty in } \\
\text { access control }\end{array}$ & Engineer & Partitioning & VMMM \\
\hline P9 & Digital access control & Engineer & Access control & VMMM \\
\hline $\mathrm{P} 10$ & Script changes & Engineer & Work instructions & VMMM \\
\hline P11 & Establish naming conventions & Engineer & Work instructions & \\
\hline $\mathrm{P} 12$ & Logging, monitoring, auditing & Engineer & Monitoring & VMMM \\
\hline $\mathrm{P} 13$ & Dual control & Engineer & Monitoring & VMMM \\
\hline
\end{tabular}

Figure 8.14: Digital operations and their contribution to goals. 


\subsubsection{Threats and Mitigations}

Having explain how entities and operations help to realize goals, we can now research threats to the realization of these goals, as well as potential mitigations.

\section{Threat Model}

The threat model used in this chapter is essentially a white box, assuming no trusted entities of the virtualization technology. As such, we depart from earlier threat models created by Vaarela [206] and VMware [36]. Since we are considering production applications, the ultimate security objective is to protect the security of the application running inside a VM (its confidentiality, integrity, availability, non-repudiation and authenticity). In our model, threats to this application can originate from five different entities: (i) hardware, (ii) other VMs, (iii) VMMs, (iv) VMMMs and (v) network.

Hardware threats are not discussed in this chapter, because these are mostly generic threats such as theft that are not specifically relevant to virtualization. More technical information on virtualization hardware is available from Perez et al [166]. Combined, this leads to the model of threats depicted in Figure 8.15.

Security claims in the literature are split over three categories: (i) analytical

\begin{tabular}{|c|c|}
\hline Threat Source & Explanation \\
\hline Network VMMM & An outsider attacks the VMMM \\
\hline Network $>$ VMM & An outsider attacks the VMM \\
\hline Network - VM & An outsider attacks the VM \\
\hline $\mathrm{VMMM} \triangleright \mathrm{VMM}$ & A VMMM attacks a VMM \\
\hline $\mathrm{VMM}$ - VM & A VMM attacks a VM \\
\hline $\mathrm{VM} \triangleright \mathrm{VMM}$ & A VM attacks a VMM \\
\hline $\mathrm{VM}$ - VM & A VM attacks another VM \\
\hline
\end{tabular}

Figure 8.15: Virtualization threats.

claims, based on logical arguments, (ii) empirical claims, based on experiments and (iii) claims based on mathematical models. The first category was dominant. Of our literature study, only one paper fitted into the third category, calculating the reliability of different virtualization architectures [101].

Figure 8.16 shows a summary of all features and their effect on security. For each feature, we show its known effect on all five security properties. A + sign indicates that the functionality provided by the feature has a positive effect on security (compared to a non-virtualized situation), without placing high demands on the environment regarding management, whereas a - indicates the opposite: the feature is technically vulnerable or can be easily misused. Furthermore a \pm indicates that the security effect depends on the particular implementation or the 
circumstances, or that the literature indicates both positive and negative effects. Finally an empty cell indicates that we could not find or deduce an effect on a security property. In the threats column of Figure 8.16 we can find which feature requires strict management because it has a potentially negative impact on security goals. Also listed are the "emergent features" (see Section 8.3.1), those features arising from unintended interactions.

The dependencies shown in Figure 8.17 shed light on which features are an essential part of the virtualization technology, and which features can be excluded in practice. As for the precise meaning of feature dependencies, we take the practical approach that given two features $A$ and $B, A$ depends on $B$ when logically it cannot function independently from $B$ without duplicating part of $B$ 's functionality. For example, the feature "Introspection" is dependent on the feature "Store VM as image". To keep the diagram clear, the dependencies of the management features are not shown, but obviously, these depend on the other features in the VMM and VMMM layer respectively.

A technological weakness can be mitigated by strong procedural security, and vice versa can a secure technology be misused in practice. Taking this into account, we have attempted to present the overall security impact of virtualization, as can be expected in practice for each of the five security properties in the next sections.

\begin{tabular}{|c|c|c|c|c|c|c|c|c|c|c|}
\hline 产 & 氖 & 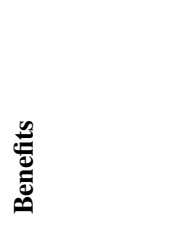 & 苞 & 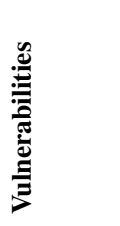 & 竧 & 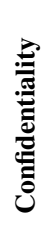 & 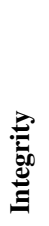 & 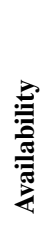 & 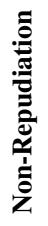 & 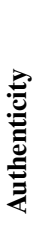 \\
\hline \multirow{2}{*}{ 茪 } & $\begin{array}{l}\text { trap program } \\
\text { execution }\end{array}$ & $\begin{array}{l}\text { facilitate } \\
\text { virtualization }\end{array}$ & N/A & N/A & N/A & + & + & + & + & + \\
\hline & TPM & $\begin{array}{l}\text { verify } \\
\text { VMM }\end{array}$ & N/A & N/A & N/A & + & + & + & + & + \\
\hline \multirow[t]{2}{*}{$\sum$} & $\begin{array}{l}\text { store VM as } \\
\text { image }\end{array}$ & backup VM & $\begin{array}{l}\text { T1: VM } \\
\text { image } \\
\text { modification }\end{array}$ & software & $\begin{array}{l}\mathrm{VMM} \triangleright \\
\mathrm{VM}\end{array}$ & - & - & + & - & - \\
\hline & $\begin{array}{l}\text { modified VM } \\
\text { software }\end{array}$ & $\begin{array}{l}\text { security } \\
\text { checks }\end{array}$ & $\begin{array}{l}\text { T2: attack } \\
\text { VMM }\end{array}$ & software & $\begin{array}{l}\mathrm{VM} \\
\mathrm{VMM}\end{array}$ & + & + & + & + & + \\
\hline \multirow{5}{*}{$\sum_{j}^{\sum}$} & $\begin{array}{l}\text { small } \\
\text { footprint }\end{array}$ & $\begin{array}{l}\text { fewer vulner- } \\
\text { abilities }\end{array}$ & & software & $\begin{array}{l}\mathrm{VMM} \triangleright \\
\mathrm{VM}\end{array}$ & + & + & + & + & + \\
\hline & $\begin{array}{l}\text { hierarchical } \\
\text { control }\end{array}$ & $\begin{array}{l}\text { control } \\
\text { untrusted VM }\end{array}$ & $\begin{array}{l}\text { T3: VMM } \\
\text { rootkit, } \\
\text { T4: VM } \\
\text { escape }\end{array}$ & software & $\begin{array}{l}\text { VM } \\
\text { VMM }\end{array}$ & + & + & + & + & + \\
\hline & $\begin{array}{l}\text { isolation } \\
\text { between } \\
\text { processes }\end{array}$ & $\begin{array}{l}\text { isolate } \\
\text { untrusted VM }\end{array}$ & N/A & $\begin{array}{l}\text { covert } \\
\text { channels }\end{array}$ & $\mathrm{VM} \triangleright \mathrm{VM}$ & + & + & + & + & + \\
\hline & logging & $\begin{array}{l}\text { store log } \\
\text { securely }\end{array}$ & N/A & N/A & N/A & & + & & + & \\
\hline & $\begin{array}{l}\text { load } \\
\text { balancing }\end{array}$ & prevent DOS & T5: DOS & software & $\mathrm{VM} \triangleright \mathrm{VM}$ & & & + & & \\
\hline
\end{tabular}




\begin{tabular}{|c|c|c|c|c|c|c|c|c|c|c|}
\hline 豙 & 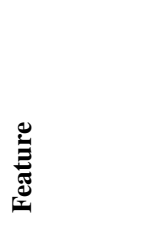 & : & $\stackrel{\mathscr{E}}{\stackrel{\mathscr{E}}{E}}$ & 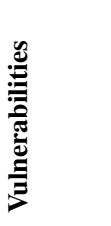 & 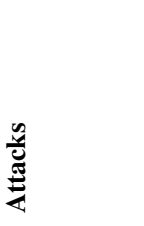 & 突 & 莺 & 㟒 & 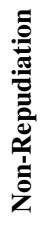 & 莺 \\
\hline & $\begin{array}{l}\text { copy and } \\
\text { backup VMs }\end{array}$ & $\begin{array}{l}\text { facilitate } \\
\text { backup }\end{array}$ & $\begin{array}{l}\text { T6: VM } \\
\text { branching }\end{array}$ & $\begin{array}{l}\text { mana- } \\
\text { gement }\end{array}$ & N/A & - & & + & & \\
\hline & introspection & $\begin{array}{l}\text { virus scan, } \\
\text { attestation }\end{array}$ & T7: misuse & software & $\begin{array}{l}\mathrm{VMM} \triangleright \\
\mathrm{VM}\end{array}$ & \pm & \pm & & & \\
\hline & attestation & $\begin{array}{l}\text { authenticate } \\
\text { VM }\end{array}$ & N/A & N/A & N/A & + & + & & + & + \\
\hline & interference & $\begin{array}{l}\text { prevent and } \\
\text { stop attacks }\end{array}$ & T7: misuse & software & $\begin{array}{l}\mathrm{VMM} \triangleright \\
\mathrm{VM}\end{array}$ & \pm & \pm & \pm & \pm & \pm \\
\hline & $\begin{array}{l}\text { power } \\
\text { functions }\end{array}$ & $\begin{array}{l}\text { recover from } \\
\text { errors }\end{array}$ & $\begin{array}{l}\text { T8: start } \\
\text { unpatched } \\
\text { VM }\end{array}$ & $\begin{array}{l}\text { mana- } \\
\text { gement }\end{array}$ & $\begin{array}{l}\text { VMM } \\
\text { VM }\end{array}$ & & & + & & \\
\hline & networking & isolation & $\begin{array}{l}\text { T9: network } \\
\text { traffic snoop }\end{array}$ & software & $\begin{array}{l}\text { VMM } \\
\text { VM, } \\
\text { network } \\
\text { VM }\end{array}$ & \pm & \pm & \pm & \pm & \pm \\
\hline & rollback & $\begin{array}{l}\text { recover from } \\
\text { errors }\end{array}$ & $\begin{array}{l}\text { T10: rollback } \\
\text { patch }\end{array}$ & $\begin{array}{l}\text { mana- } \\
\text { gement }\end{array}$ & $\begin{array}{l}\text { VMM } \\
\text { VM }\end{array}$ & - & - & + & - & - \\
\hline & $\begin{array}{l}\text { VM } \\
\text { management }\end{array}$ & $\begin{array}{l}\text { facilitate } \\
\text { control }\end{array}$ & $\begin{array}{l}\text { T11: enlarged } \\
\text { footprint, } \\
\text { T7: misuse }\end{array}$ & $\begin{array}{l}\text { mana- } \\
\text { gement }\end{array}$ & & \pm & \pm & + & \pm & \pm \\
\hline \multirow[t]{5}{*}{$\sum_{j}^{\infty}$} & transfer & $\begin{array}{l}\text { migrate if } \\
\text { error }\end{array}$ & $\begin{array}{l}\text { T5: DOS, } \\
\text { T12: } \\
\text { in-transfer } \\
\text { modification, } \\
\text { T13: transfer } \\
\text { off-site }\end{array}$ & $\begin{array}{l}\text { mana- } \\
\text { gement }\end{array}$ & $\begin{array}{l}\text { Network } \\
\text { VM, VMM } \\
\text { VM, } \\
\text { VMMM } \\
\text { VMM }\end{array}$ & - & - & + & - & - \\
\hline & replication & anti DOS & $\begin{array}{l}\text { T14: clone, } \\
\text { replicate }\end{array}$ & $\begin{array}{l}\text { mana- } \\
\text { gement }\end{array}$ & $\begin{array}{l}\text { VMMM } \\
\text { VMM }\end{array}$ & - & - & + & - & - \\
\hline & $\begin{array}{l}\text { load } \\
\text { balancing }\end{array}$ & anti DOS & T5: DOS & $\begin{array}{l}\text { mana- } \\
\text { gement }\end{array}$ & $\begin{array}{l}\text { VMMM } \\
\text { VMM }\end{array}$ & - & - & + & - & - \\
\hline & patching & $\begin{array}{l}\text { facilitate } \\
\text { patching }\end{array}$ & N/A & N/A & N/A & \pm & \pm & \pm & \pm & \pm \\
\hline & $\begin{array}{l}\text { VMM } \\
\text { management }\end{array}$ & $\begin{array}{l}\text { facilitate } \\
\text { control }\end{array}$ & T7: abuse & abuse & $\begin{array}{l}\text { VMMM - } \\
\text { VMM }\end{array}$ & \pm & \pm & + & \pm & \pm \\
\hline \multirow{3}{*}{ 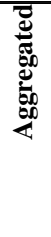 } & $\begin{array}{l}\text { loss of } \\
\text { uniqueness }\end{array}$ & N/A & $\begin{array}{l}\text { T15: mis- } \\
\text { management }\end{array}$ & $\begin{array}{l}\text { mana- } \\
\text { gement }\end{array}$ & N/A & - & & + & - & - \\
\hline & $\begin{array}{l}\text { loss of } \\
\text { location- } \\
\text { boundedness }\end{array}$ & N/A & $\begin{array}{l}\text { T15: mis- } \\
\text { management }\end{array}$ & $\begin{array}{l}\text { mana- } \\
\text { gement }\end{array}$ & N/A & - & - & + & - & - \\
\hline & $\begin{array}{l}\text { loss of } \\
\text { monotonicity }\end{array}$ & N/A & $\begin{array}{l}\text { T15: mis- } \\
\text { management }\end{array}$ & $\begin{array}{l}\text { mana- } \\
\text { gement }\end{array}$ & N/A & & - & & - & - \\
\hline
\end{tabular}

Figure 8.16: Overview of the impact of virtualization features. $(\mathrm{X} \triangleright \mathrm{Y}$ indicates an attack on $\mathrm{Y}$ from $\mathrm{X}$.) The explanation of the scores for each goal is given in Section 8.7.1. 


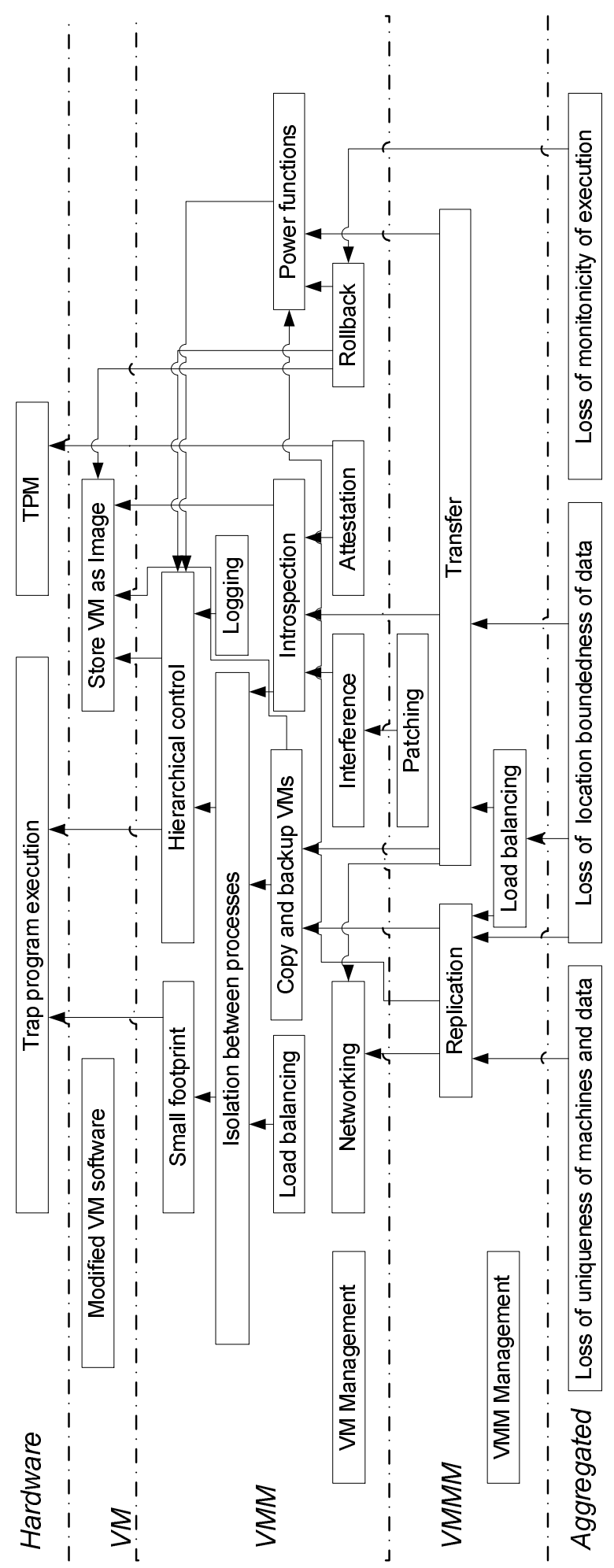

Figure 8.17: Dependencies between virtualization features. (Arrow from $A$ to $B$ indicates that $A$ depends on $B$ ) 


\section{Hardware Features and Threats}

We list two important hardware features below. For more detailed information on other virtualization hardware issues, we refer to Uhlig et al. [205] and Van Doorn [212].

Trap Program Execution The essential hardware feature enabling virtualization (present in all modern $\mathrm{x} 86$ hardware) is the ability to trap the execution of a running process and hand over control of the CPU to the VMM [22]. This allows the VMM to intervene in the execution of the process. In such a way, the VMM can perform two critical tasks: (i) emulate certain hardware and (ii) isolate the virtual machine from other running processes. This feature is an enabler for both OS and VMM security, and we consider it to have a positive effect on all security goals.

Trusted Platform Module An optional hardware feature is the Trusted Platform Module (TPM) chip, which can be used to verify that the proper VMM is indeed running, as opposed to an insecure version installed by an attacker [166]. This feature has thus a positive effect on all security goals.

\section{VM Features and Threats}

Store VM as Image VMs consist of files, holding the machine's own data, as well as some metadata (amount of memory used, number of CPUs) 7 This approach allows easy copying and modification of the VM by a VMM (improving availability), at the cost of possible confidentiality, integrity non-repudiation and authenticity problems (T1).

Modified VM Software The software running inside VMs can be equipped with so-called hooks that can be used to contact the VMM in order to execute security checks [165]. Alternative these hooks can be used to attack the VMM (T2). We consider the likelihood of such an attack small, and thus consider this feature to have a positive effect on all security goals.

\section{VMM Features and Threats}

Small Footprint Generally, the number of exploitable vulnerabilities is proportional to the amount of code. VMMs are notably smaller than the previous hardware interface layer, the operating system (OS) and are therefore deemed to be more secure [117], having a positive effect on all security goals.

\footnotetext{
${ }^{7}$ An overview of common VM data elements was created by the Distributed Management Task Force (DMTF), an IT industry consortium [49].
} 
Hierarchical Control The VMM layer is designed to control the VMs using the underlying hardware. Therefore it should not be possible for code running inside the VM to "escape" (T4) to the VMM and gain control over it. However, such escapes are possible, both in laboratory experiments [154] as well as in production environments [148]. Furthermore, the VMM is assumed to be trusted, but it also offers a layer to place a rootkit (T3). We assess that this feature has a positive effect on security because the control of the VM is given to a system with a small footprint.

Isolation Between Processes VMMs provide better isolation between virtual machines than an operating system, in which applications can normally interact [67]. However, covert channels are still an issue in existing VMM implementations [117]. Isolation between processes has a positive effect on all security goals.

Logging Virtualization can help to implement secure logging: during the execution of the VM, the VMM collects data and stores it in a place outside of the VM. Therefore it cannot be altered by an exploit that is contained inside the VM. Such a feature is implemented in ReVirt [52]. This has a positive effect on integrity and non-repudiation (while not impacting the other security goals).

Load Balancing VMMs can determine (and limit) the CPU cycles and disk space that a VM uses. This prevents a VM from starving the other VMs of resources and improves availability, without having an impact on the other security goals.

Copy and Backup VMs Making backups and copies of VMs is easier than making copies of data on physical machines. Therefore, a defective VM can be easily replaced by a working backup, improving availability. The downside is that this can lead to many different branches of VMs, which each have their own state (T6), causing integrity problems.

Introspection Since the VMM is placed at a lower level than the VM, it has the ability to "look inside the VM", to see its data and monitor its execution. This process is called virtual machine introspection [142]. The functionality can be used to run security applications such as virus scanners, intrusion detection systems such as Livewire [65], and policy checkers. Because these are outside of the VM, the VM cannot interfere with them. However, this feature also provides a new location for installing malware (T7). Therefore we assess that this feature has a mixed effect on confidentiality and integrity. 
Attestation If an inspection of a VM is performed, the VMM can send the results (the attestation) to another party. Based on the results, the receiving party can authenticate the VM, and decide whether to trust it. For optimal confidence, this function is best used in combination with trusted hardware (as in the Terra VMM [64]). This has a positive effect on confidentiality, integrity, non-repudiation and authenticity, while not impacting availability.

Interference In some cases intrusions cannot just be detected, but intervention may be possible: once the VMM detects a malicious program running inside a VM, its memory can be altered, preventing its execution [142]. Obviously this feature can also be abused by a malicious VMM (T7), and flaws in the interference mechanism could impact the VM negatively. Therefore we estimate this feature to have a mixed effect on all security goals.

Power Functions VMMs control the execution of a VM, as if it would have a virtual power button. Commonly, several functions are provided: start/power on, stop/power off, pause/suspend and reboot/reset. These functions are useful to increase availability, without impacting the other security goals. For example if a VM crashes, it can be more easily rebooted than if it were a physical machine, and it is also easier to limit resource usage by simply pausing VMs that are not needed. A danger is that VMs remain inactive for a long time and are not patched: once they are started they are vulnerable (T8).

Networking VMMs can configure the network, so that a VM only communicates with predefined VMs, forming a virtual network that does not require hardware changes. The networking functions can also be used to monitor traffic, for example running an intrusion detection system. Also this feature can be abused, for example to snoop network traffic (T9). This feature therefore has a mixed effect on all security goals.

Rollback Another feature that can be provided by a VMM is to rollback actions of a VM [66]. For example Microsoft Hyper-V has a feature to create a snapshot [136]. If a problem is detected, the VM can be restored to an earlier state, which improves availability. The danger of this feature is that patches can also be rolled back (T10), threatening the other security goals.

VM Management In order to manage the previously described features, a user interface has to be provided to control the VMM and the VMs that it runs. The management can be done from another machine (virtual or physical). For example Xen is designed to use a specific VM ("Dom0") for its management [117]. Obviously, this enlarges the small footprint that was supposed to be a strong asset of 
the VMM (T11). Management has thus a positive effect on availability (making it easier to utilize the aforementioned features), while having a mixed impact on the other security goals.

\section{VMMM Features and Threats}

Transfer VMMM can transfer ("migrate") running virtual machines between physical servers [32], [217]. This feature can be useful if a physical machine has to undergo maintenance, improving availability. Unfortunately, the feature also creates an identity problem (authenticity), for example when the network ID (often the MAC address) is reassigned randomly after the transfer [66]. Some VMMs can retain the MAC address during a live transfer [217]. If the transfer channel is not secure, the VM can be modified while in transit (T12) [151]. Improper management can also lead to the transfer of VMs to unsecure hosts (T13), to breaches of confidentiality and to denial-of-service (DOS) attacks if too many virtual machines are migrated to one VMM host (T5). As a whole, we thus estimate that the transfer function improves availability, but has a negative effect on the other security goals.

Replication Apart from being transferred, VMs can also be replicated on different physical servers [181]. This is useful to ward off a DOS attack, to distribute workload and to cope with hardware failures. At the downside, replication can be used to have VMs run on less secure locations, as was the case for the transfer feature (T14). Obviously, the security of a VM is determined by all the physical hosts it has run on. Concerning security, replication is similar to the transfer feature: it positively impacts availability while hindering the other security goals.

Load Balancing Features such as transfer and replication can be used for load balancing across different physical machines, increasing the availability of applications. Also this feature can be abused (T5), causing a denial of service attack. Again, moving virtual machines negatively impacts the other security goals.

Patching A benefit of VMMs is that they can contain software to ease the process of patching, such as VMware's vCenter Update Manager [218]. This software inspects VMs to check for missing patches. Before patching, a snapshot is made of the system. If the patching fails, the VM can revert to the snapshot. Thus VMMs make it extremely easy to rollback patches, making patching a nonmonotonic process [66].

An additional issue is that VMMs themselves also have to be patched [69], and this is more critical - if a VMM is breached, the breach might be impossible to detect because the VMM is the lowest layer of the IT stack. Patching allows to change the state of the VM, and this has a mixed effect on all security goals. 
VMM Management If several VMMs are linked together, their work needs to be coordinated from a special server, such as vCenter from VMware [218]. This gives the administrators enormous power, since they can control any VMM and any VM running on it from a single point. Another side effect is that the trusted code base is increased (similar to the management domain for a single VMM). This feature allows easier usage of the underlying functions, and we score it as having a mixed effect on all security goals, except availability, which is improved.

\section{Aggregated Features and Threats}

In the past sections, we have discussed many virtualization features, some of which have a positive effect on security, whereas others have a negative impact. In this section, we aggregate these into three emerging features that were not explicitly designed, but rather evolved from the interaction between existing features and which can be aggravated by T15: mismanagement. This high-level clustering helps to understand the effects. In total we identified three emerging features.

Loss of Uniqueness of Machines and Data In a non-virtualized server environment, applications, servers and data are to a great extent unique. However, the replication and copy/backup features reduce the uniqueness of these (improving availability), and thus having a negative impact on confidentiality, nonrepudiation, and authenticity.

Loss of Location-boundedness It is difficult to ascertain the location of a certain VM [215], since it can move between different physical servers, due to features such as transfer, replication and backup. In fact this also holds for the virtual location of a VM: if the networks are also virtualized, a VM can be accidentally dragged-and-dropped outside of a DMZ. Again, loss of location-boundedness improves availability and has a negative impact on the other security goals.

Loss of Monotonicity of Program Execution Virtualization technology causes a server's history to stop being a straight line [66]. Instead it becomes a graph, where branches are made on replication and copy operations, and a previous state can be reached when a restore is performed. Data is hard to delete as there are potentially many copies and the VM can be restored to an earlier version [66]. This threatens integrity, non-repudiation and authenticity.

\section{Summary of Threats}

Confidentiality Virtualization threatens confidentiality in several ways. First, the introspection feature gives the VMM the ability to look inside the VM. This feature can be misused or attacked. The problem is aggravated by the fact that 
VMs can be transferred between different physical servers. Obviously, replication features only increase the problem.

Integrity Together with the features of intervention and rollback comes the ability to manipulate the state of a VM, which threatens the integrity of the transactions done on a VM.

Availability The availability of applications running on VMs will most likely improve: VMs can be transferred for maintenance purposes, restored when a failure occurs and replicated if the workload requires it (Cf. Jansen et al [101].). However it must be noted that virtualization is not without availability risks, as was demonstrated by an attack on the virtualization infrastructure of the web hosting company VAServe, where 100,000 sites were deleted [200].

Non-repudiation Since virtual machines can be duplicated, rolled back and restored, there seems to be a fundamental problem regarding non-repudiation. If evidence of transactions is stored in a VM in the form of a transaction log, this can be lost if the state of a VM is restored. If transactions are signed, the key with which this is done is also stored on the VM, and can thus be copied.

Authenticity Virtualization creates identification problems. If a VM is duplicated there no longer is one original machine. Also, the identity of the machine used for communications (such as the MAC address) might change during a transfer. This ends the literature review. We will now use the framework of Figures 8.16 and 8.17 to compare digital and physical security in our case studies of virtualization.

\subsection{Properties of Digital Entities}

Figure 8.18 lists the support for the existing hypothesis and Figure 8.19 the new hypotheses discovered in the IT infrastructure case.

\begin{tabular}{ll}
\hline Property and Effect & Details \\
\hline Accurate Data Storage, & Accuracy is a precondition for virtualization. \\
Transportation and Processing & Accuracy helps to configure the system without \\
CIA+ & errors (switches, SAN, VMM). \\
& Counterexample: Data can be transported \\
& accurately outside of the system which decreases \\
& confidentiality. \\
& Conditions: Data cannot be transported out of the \\
& system.
\end{tabular}




\begin{tabular}{ll}
\hline Property and Effect & Details \\
\hline $\begin{array}{l}\text { Accurate Data Storage, } \\
\text { Transportation and Processing I- }\end{array}$ & Attacker forges system logs. \\
Fast Data Processing A- & $\begin{array}{l}\text { The VMMM infrastructure and VM images can } \\
\text { be changed fast. }\end{array}$ \\
Fast Data Storage C- & $\begin{array}{l}\text { Not observed. } \\
\text { Program Computational }\end{array}$ \\
Complexity CIA- & $\begin{array}{l}\text { Small errors impact the entire infrastructure } \\
\text { through the VMMM. }\end{array}$ \\
\hline
\end{tabular}

Figure 8.18: Support for existing hypotheses (consisting of properties and their effects) in the IT infrastructure case study. If counterexamples lead to new hypotheses they are listed individually in Figure 8.19

Accurate Data Storage, Transportation And Processing Accuracy increases the possibility for data transfer outside of the system or abuse by an attacker, negatively impacting confidentiality. An example specific to this case study is that data can be transported accurately which decreases confidentiality. There is one condition:

- The system is connected.

Fast Data Processing Fast data processing makes it easy for an attacker to examine and steal confidential data. An example specific to this case study is that a VMM can observe what is inside a VM. There is one condition:

- The system is compromised or compromisable.

In addition, fast data processing makes it easy to change a system's state and threatens integrity. An example specific to this case study is that data processing makes it easy to move between different system states (roll back and restart). There is one condition:

- The system is compromised or compromisable.

Fast Data Storage Fast data storage makes it easy to keep data available. An example specific to this case study is that easy storage on different locations increases availability. In addition to that, it makes it possible for a defender to record logs of activities that can be used to check for integrity errors and attacks, which increases integrity. An example specific to this case study is that activities for IT infrastructure management can be logged. 


\begin{tabular}{lll}
\hline Property & Effect & Hypothesis \\
\hline $\begin{array}{l}\text { Accurate Data Storage, } \\
\text { Transportation and Processing }\end{array}$ & C- & $\begin{array}{l}\text { Accuracy increases possibility for } \\
\text { transfer of data outside of system } \\
\text { or abuse by an attacker. }\end{array}$ \\
Fast Data Processing & C- & $\begin{array}{l}\text { Fast data processing makes } \\
\text { examining and stealing confidential } \\
\text { data easy. }\end{array}$ \\
Fast Data Processing & I- & $\begin{array}{l}\text { Fast data processing makes } \\
\text { changing a system's state easy. }\end{array}$ \\
Fast Data Storage & A+ & $\begin{array}{l}\text { Fast data storage makes it easy to } \\
\text { keep data available. } \\
\text { Fast Data Storage }\end{array}$ \\
It & & $\begin{array}{l}\text { Fast data storage allows a defender } \\
\text { to record logs of activities and use } \\
\text { them to check for integrity errors } \\
\text { and attacks. }\end{array}$ \\
Fast Data Transportation & A+ & $\begin{array}{l}\text { Fast data transportation makes it } \\
\text { easy to store data at a remote site. } \\
\text { Fast data transportation makes it } \\
\text { easy to store confidential data at a } \\
\text { remote, uncontrolled site. }\end{array}$ \\
\hline
\end{tabular}

Figure 8.19: New hypotheses from the IT infrastructure case study about the security effects of digital properties of entities. Each row represents either positive or negative effects. Multiple letters indicate an effect on multiple security goals. For example CIA- means a negative effect on confidentiality, integrity and availability. 
Fast Data Transportation Fast data transportation makes it easy to store data at a remote site, increasing availability. An example specific to this case study it that a system can transfer VM images off-site to increase availability. For example VM images can be transferred to other locations. This leads to one condition:

- The system is connected.

However, fast data transportation makes it also easy to store confidential data at a remote, uncontrolled site. An example specific to this case is an attacker that transfers images to other locations.

\subsection{Comparison between Physical and Virtualized IT In- frastructure}

First, we present the evaluation of physical and digital systems based on interviews with our case study participants. With these results, we contrast their security, to find the main differences and properties. We then use these properties to explain the different effects of physical and digital security.

In general, case study participants have a mixed view on physical security mechanisms. Physical security is seen as "harder" than digital security and also more static, being more difficult to change. Good physical separation requires different physical locations or rooms. However, even though separation through cables is possible, assessing if this is done properly is difficult because cables can be very long. Physical security is best for ensuring that critical data such as passwords or cryptographic keys cannot leak. Physical procedures are visible and are executed and verified by persons on location in the data center. Participants also point out that sending someone into a data center has inherent risks, as she can physically do more than she is entitled to, and this cannot always be observed easily.

In contrast digital security is considered to be "neater": it gives more control and precision for granting authorizations, and digital changes are executed directly and uniformly and can be scripted remotely. Furthermore, it allows better logging and auditing. In fact continuous monitoring is a necessity: if monitoring is not in place, virtualization causes extra risks, because the infrastructure is much more volatile, a configuration found at one time can easily have been different before, or be altered after. A drawback of digital security is also that it is not visible.

Physical controls depend on the inertness of physical systems - if untouched they remain intact. Also, physical systems can be visually inspected. By contrast, digital systems are volatile by nature; systems can reconfigure themselves to improve security, but the state itself cannot be observed directly. Physical and digital systems also differ concerning the execution of changes: physical changes require 


\begin{tabular}{|c|c|c|c|}
\hline Security Goal & Effect & Physical Property & Digital Property \\
\hline \multirow[t]{2}{*}{ All } & + & $\begin{array}{l}\text { Active, Cohesive, Dense, } \\
\text { Identifiable, Inert, Limited } \\
\text { spacious, Movable, Slow, } \\
\text { Strong, Visible, Weak }\end{array}$ & $\begin{array}{l}\text { Accurate Data Storage, } \\
\text { Transportation and } \\
\text { Processing }\end{array}$ \\
\hline & - & $\begin{array}{l}\text { Active, Movable, Strong, } \\
\text { Visible }\end{array}$ & $\begin{array}{l}\text { Program Computational } \\
\text { Complexity }\end{array}$ \\
\hline \multirow[t]{2}{*}{ Confidentiality } & + & $\begin{array}{l}\text { Difficultly manufacturable, } \\
\text { Enclosed with opening, } \\
\text { Invisible }\end{array}$ & N/A \\
\hline & - & Visible & $\begin{array}{l}\text { Accurate Data Storage, } \\
\text { Transportation and } \\
\text { Processing, Fast Data } \\
\text { Processing, Fast Data } \\
\text { Transportation }\end{array}$ \\
\hline \multirow[t]{2}{*}{ Integrity } & + & $\begin{array}{l}\text { Difficultly manufacturable, } \\
\text { Large }\end{array}$ & Fast Data Storage \\
\hline & - & N/A & $\begin{array}{l}\text { Accurate Data Storage, } \\
\text { Transportation and } \\
\text { Processing, Fast Data } \\
\text { Processing }\end{array}$ \\
\hline \multirow[t]{2}{*}{ Availability } & + & N/A & $\begin{array}{l}\text { Fast Data Storage, Fast Data } \\
\text { Transportation }\end{array}$ \\
\hline & - & N/A & Fast Data Processing \\
\hline
\end{tabular}

Figure 8.20: Comparison of physical and digital properties on their ability to realize security goals for the IT infrastructure case study. This table is constructed from data presented in Figures 8.11, 8.12, 8.18 and 8.19. 
force and are less precise than digital changes, and history of changes is harder to track than that of digital systems.

\subsubsection{Comparison Concerning Goal Realization}

We will now use the framework of Figures 8.16 and 8.17 to compare digital and physical security in our case studies of virtualization.

Our overall conclusion is that redundancy and partitioning are both good control strategies in the physical and digital world, but that access control can be more precisely regulated in the digital world than in the physical world. This confirms our conclusion from Chapter 6 that physical access control is more coarse-grained. Work instructions are not a reliable control strategy in the physical world. Monitoring is more effective in the digital world and actions can be logged easier. Figure 8.21 presents an overview of the differences with respect to goal realization. Below we explore these differences further in relation to the main security goals.

\begin{tabular}{|c|c|c|}
\hline Goal & Physical IT Infrastructure & $\begin{array}{l}\text { Virtualized IT } \\
\text { Infrastructure }\end{array}$ \\
\hline Confidentiality & + inertness of entities & $\begin{array}{l}\text { - easy introspection } \\
\text { - volatility of system } \\
\text { configurations }\end{array}$ \\
\hline Integrity & - imprecision of changes & $\begin{array}{l}+ \text { precision of changes } \\
- \text { volatility of system } \\
\text { configurations }\end{array}$ \\
\hline Availability & + inertness of entities & $\begin{array}{l}+/- \text { volatility of system } \\
\text { configurations }\end{array}$ \\
\hline Non-Repudiation & $\begin{array}{l}\text { - do not keep track of } \\
\text { history }\end{array}$ & $\begin{array}{l}+ \text { keep track of history } \\
- \text { volatility of system } \\
\text { configurations }\end{array}$ \\
\hline Authenticity & + changes require force & $\begin{array}{l}\text { - volatility of system } \\
\text { configurations } \\
+ \text { controlled changes }\end{array}$ \\
\hline
\end{tabular}

Figure 8.21: Comparison between physical and virtualized IT infrastructure. "+" and "-" indicate positive and negative effects. The explanation of the scores for each goal and system is given in Section 8.9.1.

Of these differences, the differences for confidentiality, availability and nonrepudiation are based on the literature review, whereas the differences for integrity and authenticity come from the interviews. 
Confidentiality The inertness of physical infrastructures aids confidentiality. By contrast the volatile nature of virtualized infrastructure threatens confidentiality as VMs can be transferred easily between different physical servers with different security levels. Also, replication features increase the chance of confidentiality leaks. Furthermore, the introspection feature gives the VMM the ability to look inside the VM. This feature can be misused or attacked on a much larger scale than is possible with similar features in the operating system layer on physical servers.

Integrity Together with the features of intervention and rollback comes the ability to manipulate the state and the configuration of a VM, which threatens the integrity of the transactions done on a VM. A strong point is that changes on a digital system can be made very precise. By contrast, precision is a weak point of physical IT infrastructures, as for example changing cables can be error-prone.

Availability The volatile nature of virtualizd infrastructure can have a positive effect on the availability of applications running on VMs: VMs can be transferred for maintenance purposes, restored when a failure occurs and replicated if the workload requires it (Cf. Jansen et al [101].). However it must be noted that virtualization is not without availability risks, as was demonstrated by the aforementioned attack on the virtualization infrastructure of the web hosting company VAServe. A completely virtualized infrastructure can be brought down very quickly. By contrast, the inertness of the physical components has a positive effect on the availability of physical IT infrastructures.

Non-repudiation The volatile nature of virtualized infrastructures ( the ability to duplicate, roll back and restore virtual machines) causes a fundamental problem regarding non-repudiation. If evidence of transactions is stored in a VM in the form of a transaction log, this can be lost if the state of a VM is restored. If transactions are signed, the key with which this is done is also stored on the VM, and can thus be copied. this negative impact can be offset by the fact that the history of digital changes is easy to keep track of. By contrast in a physical system, keeping track of physical changes cannot automatically lead to keeping track of a system's history.

Authenticity The volatile nature of virtualized infrastructures causes identification problems for the machines running on top of it. If a VM is duplicated there no longer is one original machine. Also, the identity of the machine used for communications (such as the MAC address) might change during a transfer. A positive aspect of virtualized infrastructures is that they can control changes very precisely, potentially making it less likely that the identity of the machines 
changes. In a physical IT infrastructure, authenticity is inherent, as changes to systems require physical force.

\subsection{Hybrid IT Infrastructure}

We now examine hybrid IT infrastructures. Contrary to the previous chapters on access control and voting, there are no specific types of systems to examine such as "location-based access control" or "optical scanners". Instead, hybrid infrastructures are simply systems that are partially virtualized. Our method to study these systems was through our case studies, and we report on the types of hybrid infrastructures that were in use there. No lists of operations or entities are given here, as these are very similar to those already shown for physical and virtualized IT infrastructure.

\subsubsection{Hybrid Systems from Case Studies}

To understand the hybrid nature of security at the case study participants, we first explain how data centers are implemented. To begin, the data center is physically divided into zones with different security levels. These zones sometimes also have different physical access controls, for example a server cabinet with a lock. Virtual machines are then only capable of migrating to and from physical servers in these zones. Within each zone, servers are physically installed and connected using cables. Administrators logically separate the systems using firewalls, and enable the necessary connections between the servers. In the products used by our participants, this is done through the definition and connection of virtual switches to different virtual machines. Management can be done using special "out-of-band channels" for remote console access. In the case of VMM servers, the management interface is digitally connected to a specific physical network card, which is then linked to the maintenance domain, routing the traffic over a specific physical network cable. Ideally, out of band access is only possible from physically secured locations.

The order of changes is significant: digital changes do normally not require physical changes, whereas a physical change would require digital reconfiguration. For example swapping a cable would require a reconfiguration of the virtual switches and firewalls. Thus, actions such as a change in the firewall policy do not require data center access, but can be done remotely. Figure 8.22 shows a part of a typical setup as found in our case studies.

Figure 8.22 shows a simplified configuration of virtualized servers as we encountered in our case studies. We have two physical servers 01 and 02 equipped with several physical network connections (NIC 01 through 05). The servers are connected through two physical switches 1 and 2 . On the physical server 01 , three 


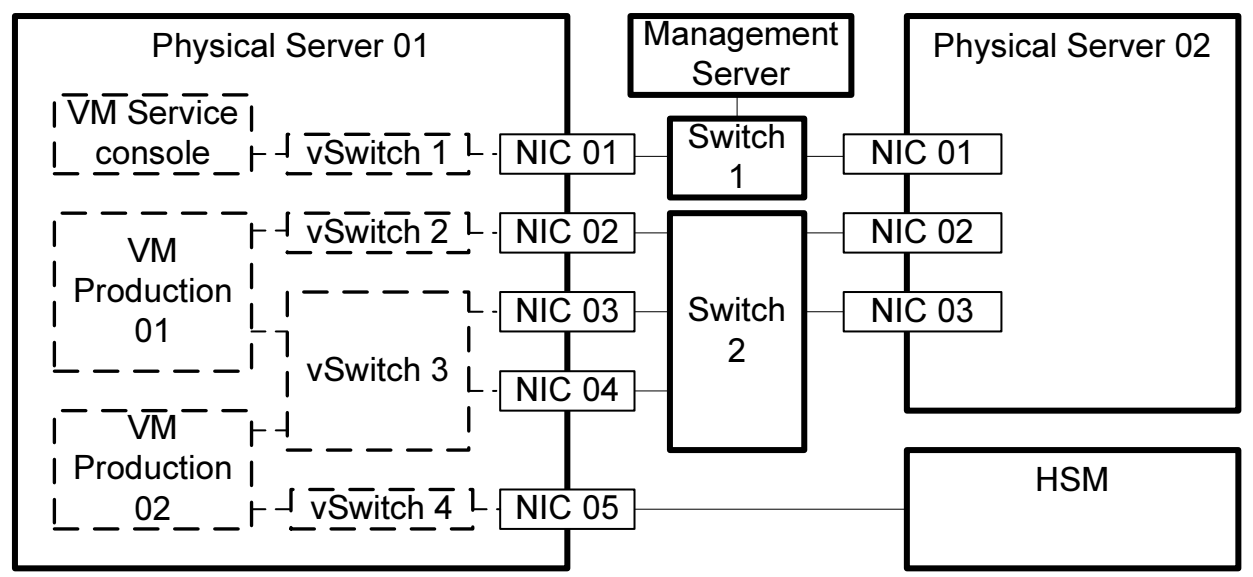

Figure 8.22: Physical and virtual connections.

virtual machines are running. Virtual machines are also connected to the network via virtual switches (vSwitch 1 through 3). Also shown is a VM that is connected to a hardware security module (HSM). These machines are of specific concern for case study participant $\mathrm{Z}$ and used for storage of cryptographic keys and the encryption and decryption of data using these keys. To prevent tampering, they are physically secured by motion detectors and light sensors. As such, a hardware security itself is a combination of hybrid security mechanisms, resulting in very tight security mechanism for storing cryptographic keys, and executing operations with these.

\subsubsection{Threats}

The results of our case studies show that the hybrid systems are not as secure as the combination might be expected to be. In fact, they are potentially less secure than normal physical IT infrastructures. If we look at Figure 8.22 , we see that the system is digitally configured such that the management console is only accessible from the management server via physical switch 1 . Thus the management network traffic is physically separated, as the normal VM traffic occurs via physical switch 1. However if the virtual switches 1 and 2 are merged by a digital change caused by an attack or human error, the management traffic can also occur via physical switch 2 and physical separation loses its value.

A similar weakness is also possible in the case of HSMs. Normally HSMs are connected to a physical server that can request the HSM to perform operations such as signing and decryption. If this is replaced by a virtualized infrastructure, as shown in Figure 8.22, the HSM has less certainty about its communication partner, as it could be any virtual machine in the network. This increases the risks for confidentiality and integrity. 


\begin{tabular}{lll}
\hline$\#$ & Explanation & Example \\
\hline S04 & Processing separation & Virtual machines running on different \\
& & processors \\
S05 & Storage separation & $\begin{array}{l}\text { Virtual machines storing data on } \\
\text { different physical systems }\end{array}$ \\
S06 & Network separation & $\begin{array}{l}\text { Virtual machines running on } \\
\text { physically separated servers }\end{array}$ \\
& &
\end{tabular}

Figure 8.23: Types of hybrid systems in the IT infrastructure case study.

\subsubsection{Types of Hybrid Systems}

We now answer RQ3 about the possible types of hybrid systems. Concerning VMs running in an infrastructure, we see three different ways to separate them physically:

- processing separation: preventing VMs running on different processors from interacting with each other.

- storage separation: preventing VMs from reading each other's data by keeping that data on separate disks or storage systems.

- network separation: preventing communication between VMs by logically keeping them on different physical servers.

\subsection{Combinations and Trade-off Analysis}

\subsubsection{Trade-off Analysis on Properties}

Having investigated all the evidence about physical, virtualized and hybrid security mechanisms, we can consider RQ4 about the trade-off analysis. Figure 8.24 and 8.25 show the trade-off analysis on the effect of physical and digital properties. For physical properties the results are mixed but for most properties there are no differences between their effects on physical and hybrid systems. Regarding digital properties, the hybrid systems can better cope with their negative effects.

\subsubsection{Trade-off Analysis on Goals}

Figure 8.26 compares hybrid systems to physical and virtualized alternatives. The results from the physical column are derived from our analysis from Section 8.7, and the virtual column from Section 8.10 . 


\begin{tabular}{|c|c|c|c|}
\hline Hypothesis & 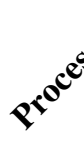 & $5^{0+0}$ & $e^{v}$ \\
\hline Active CIA+ & $=$ & $=$ & $=$ \\
\hline Active CIA- & - & - & - \\
\hline Cohesive CIA+ & $=/+$ & $=/+$ & $=/+$ \\
\hline Decaying IA- & N/A & N/A & N/A \\
\hline Dense CIA+ & $=$ & $=$ & $=$ \\
\hline $\begin{array}{l}\text { Difficultly manufacturable } \\
\text { CI+ }\end{array}$ & $=$ & $=$ & $=$ \\
\hline Easily manufacturable CI- & N/A & N/A & N/A \\
\hline Enclosed with opening $\mathrm{C}+$ & $=$ & $=$ & $=$ \\
\hline Identifiable CIA+ & - & - & - \\
\hline Inert CIA+ & - & - & - \\
\hline Invisible $\mathrm{C}_{+}$ & $=$ & $=$ & $=$ \\
\hline Large I+ & $=$ & $=$ & $=$ \\
\hline Limited spacious CIA+ & $=$ & $=$ & $=$ \\
\hline Modifiable CI+ & $=$ & $=$ & $=$ \\
\hline Modifiable CI- & $=$ & $=$ & $=$ \\
\hline Movable CIA+ & $=/-$ & $=/-$ & $=/-$ \\
\hline Movable CIA- & $=/+$ & $=/+$ & $=/+$ \\
\hline Slow CIA+ & $=/-$ & $=/-$ & $=/-$ \\
\hline Strong CIA+ & $=/+$ & $=/+$ & $=1+$ \\
\hline Strong CIA- & $=/+$ & $=/+$ & $=/+$ \\
\hline Visible C- & N/A & N/A & N/A \\
\hline Visible CIA+ & - & - & - \\
\hline Visible CIA- & - & - & - \\
\hline Weak CIA+ & $=$ & $=$ & $=$ \\
\hline
\end{tabular}

Figure 8.24: Trade-off analysis on the impact of properties between hybrid and physical for the IT infrastructure case study. Positive scores indicate that the hybrid system is more secure than the physical system, negative scores that it is not. N/A means that the effect was not observed in the case study. (For the exact operationalization details, see Figure 5.11 in Chapter 5.) The full explanations of the scores for each goal are Section 8.11 


\begin{tabular}{|c|c|c|c|}
\hline Hypothesis & $\mathbf{s}^{e^{e^{e}}}$ & $500^{5}$ & $\vec{r}^{\mathbf{v}}$ \\
\hline $\begin{array}{l}\text { Accurate Data Storage, } \\
\text { Transportation and } \\
\text { Processing C- }\end{array}$ & $=$ & $=$ & $=$ \\
\hline $\begin{array}{l}\text { Accurate Data Storage, } \\
\text { Transportation and } \\
\text { Processing CIA+ }\end{array}$ & $=$ & $=$ & $=$ \\
\hline $\begin{array}{l}\text { Accurate Data Storage, } \\
\text { Transportation and } \\
\text { Processing I- }\end{array}$ & $=/+$ & $=/+$ & $=/+$ \\
\hline Fast Data Processing A- & $=/+$ & $=/+$ & $=/+$ \\
\hline Fast Data Processing C- & $=$ & $=$ & $=$ \\
\hline Fast Data Processing I- & $=$ & $=$ & $=$ \\
\hline Fast Data Storage A+ & $=$ & $=$ & $=$ \\
\hline Fast Data Storage C- & $=$ & $=/+$ & $=/+$ \\
\hline Fast Data Storage I+ & $=$ & $=$ & $=$ \\
\hline Fast Data Transportation A+ & $=$ & $=$ & $=$ \\
\hline Fast Data Transportation C- & $=$ & $=$ & $=/+$ \\
\hline $\begin{array}{l}\text { Program Computational } \\
\text { Complexity CIA- }\end{array}$ & $=/+$ & $=/+$ & $=/+$ \\
\hline
\end{tabular}

Figure 8.25: Trade-off analysis on the impact of properties between hybrid and digital for the IT infrastructure case study. Positive scores indicate that the hybrid system is more secure than the digital system, negative scores that it is not. N/A means that the effect was not observed in the case study. (For the exact operationalization details, see Figure 5.11 in Chapter 5) The full explanations of the scores for each goal are Section 8.11 .

\begin{tabular}{lcc}
\hline Goal & Hybrid Compared to Physical & Hybrid Compared to Virtual \\
\hline Confidentiality & - & $=/+$ \\
Integrity & \pm & $=/+$ \\
Availability & + & $=/+$ \\
Non-repudiation & - & $=/+$ \\
Authenticity & - & $=/+$ \\
\hline
\end{tabular}

Figure 8.26: Comparison of hybrid with physical and virtualized systems. (For the exact operationalization details, see Figure 5.11 in Chapter 5.) The explanation of the scores for each goal is given in Section 8.11 
Hybrid systems score as good as fully virtualized systems on all security properties, and likely better, because they can make use of the advantages of both physical and digital systems. Compared to physical systems, hybrid systems score worse on the goals of confidentiality, non-repudiation and authenticity because they are more volatile and their configuration is less stable and can easier expose data. For integrity, the advantages are less clear: hybrid systems can improve integrity, because changes are executed more precise, but they can also be negatively affected by the fact that they are more volatile. Finally the availability of a hybrid system is better than that of a physical system, because data and systems can be more easily replicated and migrated.

\subsection{Conclusion}

\subsubsection{Physical and Digital Properties}

There are three relevant properties of physical entities. First, physical infrastructure is visible and inspectable by persons. This provides assurance about the system's configuration. Second, the inertness property assures that a given configuration remains in place after inspection. Third, persons configuring infrastructure are slow, and this adds confidence that IT system configurations remain in place (even if they are incorrect). As for digital properties, they allow for accurate changes but also for flexibility that can be used against the system.

\subsubsection{Comparison between Physical and Virtualized IT Infrastructure}

Concerning trade-offs, physical mechanisms are especially beneficial when few changes are made to systems, whereas digital mechanisms are preferred when systems are very dynamic. However, digital misconfigurations are always possible, and for every physical security mechanism to be replaced by a digital mechanism, the monitoring and auditing requirements increase substantially.

\subsubsection{Types of Combinations}

Regarding the combinations of physical and digital security, we learned that existing technology limits effective combinations of physical and digital security mechanisms. Currently, these combinations lead to an overly heavy reliance on digital security mechanisms, negating positive effects of physical security. For example, even if traffic is initially routed over different physical networks, the VMM (a software component) can undo this security measure and change the routing. 


\subsubsection{Trade-off Analysis}

As for trade-off analysis, hybrid systems are as good as completely virtualized systems (or better). Compare to physical systems, their availability is improved, at the expense of confidentiality and integrity.

\subsubsection{Security Impact of Complete Virtualization}

Finally we answer the specific research question $\mathbf{R Q 5} 5_{\text {infra }}$ about the impact of complete virtualization. From the comparison in Section 8.9, we believe that there is a fundamental limit as to what safely can be virtualized. A network where the audit trails of the VMMs, the identity of the administrators, and the DNS servers are all virtualized, lacks a single system from which to build trust as all systems becomes fluid. The more systems are virtualized, the more they are to lose properties such as uniqueness, location boundedness and monotonicity, which are important to security.

As for the underlying physical properties that affect security, we found that virtualization security depends heavily on the ability to trap program execution and hierarchical control of the VMM. Digital security mechanisms facilitate (but also require) monitoring and auditing. Core features of virtualization (isolation between processes and small footprint) have clear positive effects on most security goals, but it is clear that these effects are mitigated by newer features built on top of these. They make the VMM layer bloated [22], increasing the likelihood of bugs, giving much more control to the administrators. As a whole, virtualization has a positive effect on availability but threatens confidentiality, integrity, authenticity and non-repudiation, even though many features are designed with these goals in mind. These findings have consequences for our view on cloud computing, which depends heavily on virtualization technology; Ensuring confidentiality in a cloud environment requires more effort compared to a non-virtualized environment.

If we consider the HSMs from Section 8.10, we can hypothesize about the security if such systems were virtualized themselves. How secure would data in a virtualized HSM be? Given the volatility of virtual machines, the consequence of HSM virtualization would be that the secrets enclosed in them could be spread over the entire data center. Therefore the strict security requirements that are placed on the HSM must be extended beyond the original HSM to the entire data center. Obviously, a HSM is easier to manage and this indicates a limit of the benefits of virtualization.

A more fundamental argument to the question of virtualization's limits can be given by observing that both availability and confidentiality have their roots in the physical environment: confidentiality as in the case of HSMs data depends on keys (which must be physically and digitally confined). By contrast, availabil- 
ity of data requires multiple data centers in different locations and connections between them, to ensure continuous operation. As such there is an inherent conflict between these two, and virtualization (connecting and integrating systems) naturally favors availability over confidentiality. 


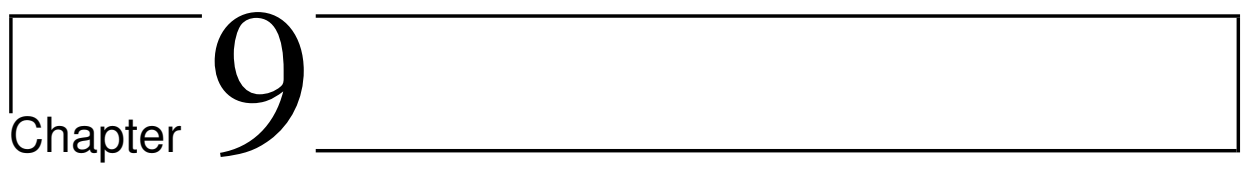

\section{Rights Management Systems}

\subsection{Introduction}

This chapter covers the cases concerning systems that limit the usage of content, for example music or written text. We call the systems that enforce copyright or limit content usage "rights management systems". There are several reasons why this is an interesting class of systems to study in the context of our investigation of physical and digital security mechanisms.

A first reason is that the same types of content (movies, documents, games) are often available in both digital and physical form. For example, a movie can exist on celluloid or as bits on a computer hard disk. This allows us to compare goal realization between physical and digital systems. Digital Rights Management (DRM) systems are commonly understood to be those systems that limit the usage of digital content, such as music in MP3 form, a movie available on a website or an e-book. Similarly, physical or analog types of content also exist such as printed books, and the difficulties to make good copies of them helps to reduce piracy. Hybrid protection mechanisms also exist, which employ a combination of physical and digital security mechanisms. For example an e-book can be encrypted such that it can only be rendered on a specific physical device. (We will return to the definitions of DRM and hybrid mechanisms in Section 9.2.)

A second reason why rights management systems are a particularly good case study is because they have been plagued by security problems. Many DRM solutions have failed in the past, and the effectiveness of DRM is constantly questioned [54]. Schneier (2001) summed up the arguments against DRM as follows: "Digital files cannot be made uncopyable, any more than water can be made not wet" [189]. However, since then, many new DRM solutions have been devised to do just that, and recreate the limitations that exist in the physical world for making copies. For example the IEEE workgroup has proposed the P1817 Standard 
for Consumer-ownable Digital Personal Property [89]. The special hardware proposed by this standard does not prevent copying of the contents itself, but allows only one copy to be used at the same time. Apple's DRM solutions for applications running on the iPad and iPhone have been very successful as its "App Store" generated billions of dollars in revenue [7].

Still, the analog hole represents a fundamental limitation of DRM techniques. All digital content is ultimately presented to persons in an analog form and this representation can always be copied. For example a digital movie can be secured through various DRM techniques that only allow it to be played on specific devices. However, these devices must eventually show the movie on a screen from which it can be recorded by a video camera.

Our approach to understanding physical and digital security for content is similar to that of the other case studies: we will start to create the KAOS tree by listing the technology independent goals of RM (rights management). Next we discuss the physical, digital and hybrid systems implementations. In between these sections the research questions are answered about physical and digital properties (RQ1 and RQ2), the comparison between physical and digital (RQ3), the types of hybrid systems (RQ4) and finally the trade-off analysis (RQ5). Contrary to the previous case study in Chapter 8, this study is meta research: we only study literature and do not visit any particular organization.

A specific topic of interest in this chapter is music: both researchers and the content industry have spent a significant effort in attempting to develop RM systems that protect it, but so far these efforts have failed. Therefore we also attempt to answer special research question $\mathbf{R Q} \mathbf{4}_{\mathrm{RM}}$ : can hybrid methods effectively restrict the usage of music?

\subsection{Conceptual Framework}

\subsubsection{Definition and Concepts}

Every word in the acronym "DRM" is subject to discussion [124]. First, concerning "digital", there is discussion about whether "digital" concerns the management or the rights. In other words, is DRM "digital management of rights" or "management of digital rights"? In this chapter, and in line with the earlier chapters where physical, digital and hybrid systems were discussed, we consider DRM to be the intersection of these two definitions: DRM is the "digital management" of "digital content", it protects for example MP3 files and computer games through digital means. Vice versa, PRM (Physical Rights Management) is the management of physical (or analog) content such as a printed book or music on an LP through physical means. This leaves a third category of HRM (Hybrid Rights Management): the combination of physical and digital means to either secure digital or physical content. 
A second topic of discussion concerns the meaning of the "rights" of DRM. DRM is often described as protecting "content" or "intellectual property" (IP). However, the terms content and IP are not equal terms, as IP also can also refer to patents. We view DRM as protecting content, and interpret content in a wide sense, also including products and services such as video games.

Third, one can argue that the term "management" in DRM is too narrow, as DRM systems not only concern managing but also enforcing rights to content [124]. We agree with this, and view enforcement as necessary part of any RM system. Thus, Figure 9.1 illustrates our point of view and shows the types of $\mathrm{RM}$ systems in relation to the types of contents.

\begin{tabular}{rll}
\hline RM System & Content & Mechanism \\
\hline Physical RM & Physical & Physical \\
Digital RM & Digital & Digital \\
Hybrid RM & Physical & Physical and digital \\
& Digital & Physical and digital \\
\hline
\end{tabular}

Figure 9.1: Physical, digital and hybrid rights management systems.

Having discussed the meaning of "DRM", we now investigate its technological aspects. $\mathrm{Ku}$ and $\mathrm{Chi}$ performed a detailed survey on these and identified five components [124]:

1. unique identifiers and meta-data

2. digital watermarks

3. digital fingerprints

4. secure containers

5. rights expression languages

To begin, unique identifiers are necessary to identify the content itself, such as an ISBN number for a book, as well as users. Whereas digital watermarks are inserted purposely into the content to identify it at a later stage, fingerprinting is a technique to identify content without such watermarks being present. For example a bootleg video of a live concert is placed on a website, and a fingerprinting algorithm determines that it is a recording of a specific song from an artist. A well-known example of fingerprinting is the service Shazam [222], which allows users to send a short recording of a song via their mobile phone, and receive information about the song such as the artist's name. DRM also requires secure containers that store and process the content while enforcing the rights. These are realizable using a Trusted Computing Base (TCB) that integrates cryptographic 
controls and mechanisms on a special hardware chip. Finally rights expression languages are necessary to define how the content usage should be restricted. Of these components, only secure containers has a clear link with hybrid rights management (Section 9.10), whereas the other components are digital protection mechanisms (Section 9.7). As for the UCON framework presented in Chapter 6 (1) is used by models, architectures and mechanisms. (2), (3) and (4) clearly fit into the UCON mechanism category. Finally, (5) fits into the model category.

\subsubsection{History and Context of Rights Management Systems}

\section{Rights Management, Reproduction and Authenticity}

A RM system is used to control access to content, such as books or music. This implies limiting the options for their reproduction. Benjamin (1936) placed reproductive capabilities of art (and more general of content) in historical perspective [15]. Works of art are valuable objects, and this has stimulated technical innovations that give more people access to them. Thus, subsequent technologies such as founding, stamping, printing, etching, photography and film have made it easier to reproduce works of art, even before the introduction of digital media. Rather than visiting a museum to view a painting, we can observe a photo of it instead. The increase in reproductive capabilities has several important consequences:

first, the increase in reproductive capabilities, especially with respect to the shift from physical to digital content, has threatened the authenticity of art. If a perfect copy can be made through the same technical process that created the original, the original loses its value. For example, all photos created from the same negative are considered to be identical and this diminishes their value. The problem of authenticity becomes clear if we consider an auction of private letters. We can auction letters written on paper by a famous writer because they are unique. By contrast, auctioning her emails would clearly raise questions: as there are no original emails, how can they be sold? What is the original, the email in the sent items box of the author or the one in the inbox of the receiver? Surely the emails only have value insofar as there is no other copy available to the public. By contrast, detailed knowledge about a painting (such as the high resolution scans from the Google Art Projec11] cannot cause any loss of authenticity.

As for information security, authenticity is one of the security goals mentioned in ISO 13335 [91] (Cf. Chapter2), defined as [sic] the property that ensures that the identity of a subject or resource is the one claimed. For a digital resource, authenticity has been given a different meaning: it is now best understood as "unaltered after the creation by a certain subject", regardless of the number of copies in existence. As such authenticity is strongly related to integrity:

\footnotetext{
${ }^{1}$ http://www.googleartproject.com
} 
- A digital photo is authentic to the extent that it has not been tampered with after the camera saved it.

- A digitally signed PDF document is authentic to extent that it is created by the author and was unaltered after that.

Second, with the loss of authenticity, the concept of forgery also changes: a painting made by Picasso can by definition not be copied perfectly, it can only be forged. Only an original Picasso painting is painted by his hand, and is now on display in a museum. By contrast, a technical reproduction of a photo from a negative is simply the same photo. The claim that a perfect copy of a digital file is a forgery is absurd. Similar to the change in the meaning of authenticity, a digital forgery can now be understood as a secret alteration of an existing file.

Third, new technology has resulted in the creation of new types of art. For example, a photo of a painting is a reproduction, but photography itself is also a new art form. In turn, the invention of newer art forms that are less authentic and easier to reproduce created a demand for controlling technologies. As digital forms of art (such as computer games) are the easiest to reproduce, digital technologies led to digital rights management.

\section{Forces Affecting Human Behavior in Cyberspace and Real Space}

Concerning the reproductive capabilities, Lessig shares Benjamin's analysis of the past technological developments, but argues that the first breakthrough did not arrive until the introduction of digital audio technology (DAT) tapes in the 1970s [129]. The contents of a DAT tape is perfectly copyable, whereas a copy of an analog tape always has a lesser quality than the "original". In the United States, pressure from the music industry led to legislation and imposition of technical limits. First a tax on blank tapes was introduced and second the number of copies that could be made from one tape was limited through a hardware solution.

In a crude way, the latter thus recreated the imperfections that existed in the physical world. However, the physical architecture of space still limited the physical distribution of DAT tapes and this hindered the actual ability of persons to copy them. This changed with the Internet, which allowed easy distribution of the digital content.

Concerning the differences between control in cyberspace and physical space (our research question RQ2), Lessig's thesis is that human behavior is influenced by four forces (laws, architecture, markets and norms) and that these forces work differently in each space. We will now explain these forces for rights management.

- Architecture makes certain actions possible or impossible. In the terms of Chapter 5 (concerning the interaction of a system with the context), we define architecture as a collection of components with capabilities connected 
through interaction mechanisms. These components can be either physical, hybrid or digital.

For example, in "real space" it is impossible to steal a skyscraper because of its size. By contrast the basic architecture of cyberspace is such that reproduction is easy. Implementing DRM into this architecture can however limit copying, but yet other forms of architecture (or code) can negate DRM. For example P2P filesharing protocols facilitate copying even in the presence of DRM.

- Regulation is also a factor in copyright protection, for example the Digital Millennium Copyright Act (DMCA) adopted by the United States in 1998, criminalizes the reverse engineering of copyright protection schemes.

- Market forces, the decisions that corporations make about their products, also influence the enforcement of copyright. For example low-priced content is a stimulus for consumers to accept DRM systems.

- Social norms about copyright also play a role. The idea of ownership is deeply embedded in society (at least in the developed world), and this idea relates strongly to the belief that persons can do with their belongings as they see fit. Regardless of any contractual obligations, persons feel thus free to distribute content further, once they "own" it.

Lessig is not convinced that technological development will continue to make copying and distribution easier. Architecture (physical, digital or both), regulation, market forces and social norms can all change. Particularly concerning architecture, new technologies are being developed under the name of "trusted systems": system that use a combination of physical and digital protection mechanisms (hybrid mechanisms in our terms) to regulate user behavior with great precision and limit the usage of copyrighted works of art. In this chapter, we will concentrate on architectures.

\subsubsection{Summary and Implications for Case Study}

We investigated the meaning of DRM and presented physical and hybrid counterparts to it. The related work discussed before places DRM systems in historical perspective and explains the context in which they function. We discovered the importance of authenticity in relation to content protection, as well as its different meaning for physical and digital content. Furthermore, technology is not the only determinant concerning the effectiveness of rights management systems, and a choice must be made concerning the scope of the study. This is done in the next section, where we explain our research method. 


\subsection{Method}

\subsubsection{Case Study Selection and Method}

Similar to our other case studies, we examine digital, physical and hybrid rights management systems. We perform a multiple case study on a variety of systems for four types of content: text, music, games and sports matches. These cases vary widely in the types of rights management that are used, as well as their success in protecting rights, thus achieving a good theoretical spread. Some of the investigated systems (such as games) can be physical, digital or hybrid. Others, such as live events only fall in one category (in this case physical rights management). Figure 9.2 summarizes our cases. Each case study consists of a literature study, with data found through a search on Scopus based on keywords searches of "DRM" and "rights management". We now explain our cases in more detail.

\begin{tabular}{llll}
\hline Content & Physical System & Digital System & Hybrid System \\
\hline Text & S1. Book & S2. PDF & S3. E-reader and e-book \\
\hline Music & S4. Live music & S7. Internet radio & S9. Portable media player \\
& S5. Tape recorder & S8. PC and MP3 player & \\
& S6. Radio broadcast & & \\
\hline Game & S10. Board game & S11. PC game & S12. Online console game \\
\hline Sports & S13. Live match & S16. Live stream & S18. Pay-per-view \\
match & S14. Video recorder & S17. Video-sharing site & \\
& S15. TV broadcast & & \\
\hline
\end{tabular}

Figure 9.2: Overview of cases.

- Texts: Our first case concerns IP in the form of text. We begin with traditional literature in book form, PDF documents and end with hybrid ereaders and e-books, such as the Amazon Kindle 2 .

- Music: Our second case involves music. Again, we start with original reproduction from music sheets by an orchestra (a live event), to modern ways of reproduction via MP3 files.

- Games: The third case study concerns gaming. Traditionally, IP protected games were played on a board, such as Monopoly or Risk. Board games were partially replaced by computer games, distributed via floppy disks, CDROMs or special cartridges. Games are now increasingly played over the Internet, connecting to servers of the game developing companies. Some

$\sqrt[2]{\text { http: //kindle.amazon.com }}$ 
online games are only playable using special consoles, such as the Sony Playstation that connects to the Sony Playstation Network.

- Sports Matches: The fourth case is sports matches. Before broadcasting technology was available, the only means to observe a live event was to witness it at the same location: one had to be in the stadium to watch a football match. Next, these events were broadcast over the air, on radio or TV channels. Currently, broadcasting is also done via the Internet and with specific rights management mechanisms, such as Eredivisie Live $\}^{3}$, the pay-tv channel of the Dutch national football division.

Another categorization is possible by distinguishing between the types of interaction that the consumer has with the systems, because this determines their security. We can thus group the systems from Figure 9.2 in the following four categories.

- Live event: includes all cases where a person creates content live for an audience at the same location. $(S 4, S 13)$

- Recording: includes all cases where content is recorded on a medium. ( $S 1$, S2, S3, S5, S8, S9, S14, S17)

- Game: includes the case where consumers play a game. (S10, S11, S12)

- Broadcast: includes cases where consumers receive content passively. (S6, S7, S15, S16, S18)

\subsubsection{Limitations and Scope}

Our research subject of rights management systems is very large and has many related areas of research, all of which could yield potentially interesting information. As explained by Lessig (Section 9.2 RM involves legal, social and technical issues. We cannot explore all of these in-depth. Below we summarize the limits of the scope of our research, and consider unexplored opportunities.

First, as our research does not focus on law, we will not elaborate on the different issues concerning copyright protection, patents, intellectual property. Neither will we investigate different business models for generating revenue from content, and compare these to the effectiveness of DRM: our focus is on technical mechanisms to protect content.

Second, there is a substantial body of work in closely related areas. To begin, DRM techniques have similarities with data-loss prevention techniques (DLP) [126] used to prevent sensitive data from leaving an enterprise's systems. However, we will not investigate DLP in this chapter as we examine content protection

$\sqrt[3]{\text { WwW } \text {. eredivisielive.nl }}$ 
for consumers. Furthermore, the goals of DRM of retaining control over data are similar to those of individuals who wish to protect their data and their privacy [5]. Nevertheless, we will not include privacy enhancing technologies (PET) in our research.

Third, as we mentioned in Section 9.2, hybrid systems are those that use a combination of physical and digital mechanisms to either secure physical or digital content. In this chapter we will however not examine the protection of physical content through digital means, but rather focus on digital content and how physical mechanisms can improve its security. The reason for this choice is that the latter has been the focus of existing DRM research to which we hope to contribute.

\subsection{Goals}

We will now explore how the goals of rights management fit in our conceptual framework from Chapter 2 and begin to build the KAOS model. Figure 9.3 shows a high-level breakdown of the goals of a content producer.

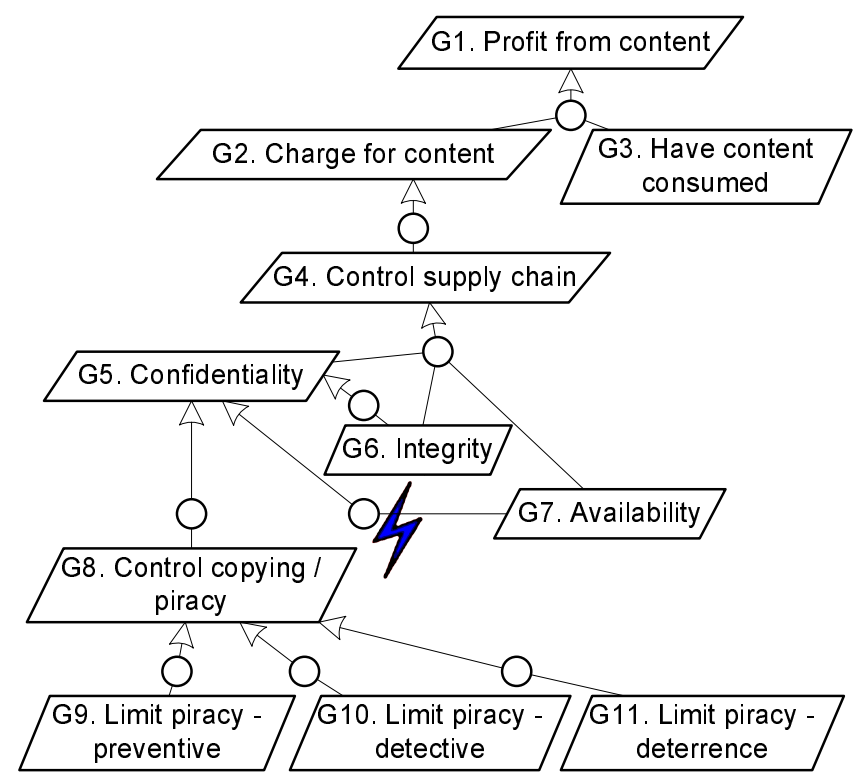

Figure 9.3: Top-level goal tree for rights management.

The goal tree is based on our literature study [129], [141], [152], [161]. We explain the structure in more detail below. To begin, for a content producer using rights management, the top level goal is to maximize revenues from contents (G1) by charging for it (G2) and making sure it is actually consumed (G3) (As mentioned in Section 9.3 we exclude other revenue models from our analysis.) Charging content is only possible if the supply chain is controlled (G4) and all 
its security aspects of confidentiality, integrity and availability (G5, G6 and G7). The most important goal conflict is between confidentiality and availability: in order to maximize revenue from content it must be made available, and this implies some loss of confidentiality. Note that confidentiality in the context of rights management does not mean that information is secret. A movie is not secret, the distributer simply wants to be paid for someone viewing it. Thus confidentiality is best understood as limited availability, similar to the definition of confidentiality in ISO 7498 (Chapter 2): the property that information is not made available or disclosed to unauthorized individuals, entities, or processes.

Important for rights management is that integrity has a positive effect on confidentiality: when content is kept in a specific format it is easier to control (for example through water marking). As for the security goal of authenticity mentioned in Section 9.2, we do not see this as a being goal of rights management, instead it is a property of content; we cannot make content authentic - either it is authentic or it is not. Moving further down the goal tree, the goal of confidentiality is realized by controlling copying/privacy (G18), which is split in preventive, detective and deterrence controls (G9, G10, G11).

\subsection{Physical Rights Management}

Having presented the technology independent goals, we now examine how they can be realized, starting with physical systems. Figure 9.4 lists the different entities for each case. These will later be used to find the physical properties (RQ1) relevant for RM.

Figure 9.5 shows the operations. The operations are divided between the operations performed by the producer (goals beginning with "P") and by the consumer ("C"). The producer's operations consist of a pattern with five general operations: create content, protect content, distribute content, monitor usage and receive payments. The consumer's pattern is to buy content, prepare it for usage and finally consume it. 


\begin{tabular}{|c|c|c|c|}
\hline Entity Type & ID & Entity & Case (System) \\
\hline \multirow[t]{3}{*}{ Medium } & E1 & Book & Text (S1) \\
\hline & $\mathrm{E} 2$ & Tape & Music (S5) \\
\hline & E3 & Board game & Games (S10) \\
\hline \multirow[t]{3}{*}{ Player } & E4 & Tape recorder & Music (S5, S14) \\
\hline & E5 & Radio & Music (S6) \\
\hline & E6 & TV & Sports match (S15) \\
\hline Recorder & E7 & Tape recorder & Music (S5, S14) \\
\hline \multirow[t]{3}{*}{ Performers } & E8 & Singer & Music (S4) \\
\hline & E9 & Soccer team & Sport match (S13) \\
\hline & E10 & Soccer player & Game (S10) \\
\hline \multirow[t]{3}{*}{ Location } & E11 & Music hall & Music (S4) \\
\hline & E12 & Stadium & Sports match (S13) \\
\hline & E13 & Home & $\begin{array}{l}\text { Text, Music, Game, Sports match } \\
(\mathrm{S} 1, \mathrm{~S} 5, \mathrm{~S} 6, \mathrm{~S} 10, \mathrm{~S} 14, \mathrm{~S} 15)\end{array}$ \\
\hline Consumer & E14 & Consumer & Text, Music, Game, Sports match (all) \\
\hline Ticket & E15 & Entry ticket & Music, Sports match (S13) \\
\hline
\end{tabular}

Figure 9.4: Entities in physical rights management systems.

\begin{tabular}{|c|c|c|c|c|c|c|}
\hline ID & Operation & Live Event & Recording & Game & Broadcast & Goals \\
\hline P1 & Create & Perform & $\begin{array}{l}\text { Create } \\
\text { recording }\end{array}$ & Design game & $\begin{array}{l}\text { Create } \\
\text { content }\end{array}$ & G4 \\
\hline $\mathrm{P} 2$ & Protect & Fence site & $\begin{array}{l}\text { Manufacture } \\
\text { recording }\end{array}$ & $\begin{array}{l}\text { Manufacture } \\
\text { game }\end{array}$ & - & $\begin{array}{l}\text { G5, } \\
\text { G6 }\end{array}$ \\
\hline P3 & Distribute & $\begin{array}{l}\text { Travel to } \\
\text { site }\end{array}$ & $\begin{array}{l}\text { Distribute } \\
\text { record }\end{array}$ & $\begin{array}{l}\text { Distribute } \\
\text { game }\end{array}$ & $\begin{array}{l}\text { Broadcast } \\
\text { content }\end{array}$ & $\begin{array}{l}\text { G3, } \\
\text { G7 }\end{array}$ \\
\hline P4 & $\begin{array}{l}\text { Monitor } \\
\text { usage }\end{array}$ & Guard site & - & - & - & $\begin{array}{l}\text { G9, } \\
\text { G10, } \\
\text { G11 }\end{array}$ \\
\hline P5 & $\begin{array}{l}\text { Receive } \\
\text { payment }\end{array}$ & $\begin{array}{l}\text { Receive } \\
\text { payments }\end{array}$ & $\begin{array}{l}\text { Receive } \\
\text { payments }\end{array}$ & $\begin{array}{l}\text { Receive } \\
\text { payments }\end{array}$ & $\begin{array}{l}\text { Receive } \\
\text { payments }\end{array}$ & $\mathrm{G} 2$ \\
\hline $\mathrm{C} 1$ & Buy & Buy ticket & $\begin{array}{l}\text { Buy } \\
\text { recording }\end{array}$ & Buy game & $\begin{array}{l}\text { Buy } \\
\text { receiver }\end{array}$ & $\mathrm{G} 2$ \\
\hline $\mathrm{C} 2$ & Prepare & $\begin{array}{l}\text { Travel to } \\
\text { site }\end{array}$ & $\begin{array}{l}\text { Place } \\
\text { recording in } \\
\text { player }\end{array}$ & $\begin{array}{l}\text { Unpackage } \\
\text { game }\end{array}$ & $\begin{array}{l}\text { Tune in on } \\
\text { transmitter }\end{array}$ & G3 \\
\hline $\mathrm{C} 3$ & Consume & $\begin{array}{l}\text { Observe } \\
\text { event }\end{array}$ & $\begin{array}{l}\text { Consume } \\
\text { recording }\end{array}$ & Play game & $\begin{array}{l}\text { Receive } \\
\text { broadcast }\end{array}$ & G3 \\
\hline
\end{tabular}

Figure 9.5: Operations of physical rights management systems and their contributions to goals. 


\subsubsection{Threats and Mitigations}

Figure 9.6 shows the threats to physical systems. Live events such as sports matches link the content consumption to a particular location. This allows the usage of the content to be controlled, by ensuring that persons pay at the entrance. Physical access protection mechanisms (walls, fences, turnstiles) can help to prevent people from sneaking inside (T2). (See also Chapter 6 on Access Control.) Still, ticket forgery remains possible (T1). Furthermore, visitors can record content (T3). Specific for live events this used to be difficult because recording equipment was very large (tape recorders etc.) and easily detectable. However this has become easier as these devices have become smaller. A broadcast of music or sports matches can also be recorded. In contrast to recordings of a live event, it is more difficult to prevent this copying, because the content producer cannot monitor the consumer's behavior. Another threat is rebroadcasting of content, a pirate radio station can broadcast music without permission (T4). Specific for recordings, content recorded on a medium can be stolen (T5), leading to a loss of control over the supply chain.

Content can also be copied (T6). As for music on tape and printed books, they can be copied on paper by reprinting them. This can be cumbersome and require a copy of the book itself. Copy machines provide further automation of the copying process [129, p. 172], although the copies are of lower quality than the original.

A board game requires physical manufacturing in a factory. An attacker would have to recreate the board, the board pieces and the manuals (T7), requiring a complicated machine, limiting the opportunity for end-users. Large scale manufacture is detectable because the board games require transportation. For example checks at customs can find counterfeit products.

\begin{tabular}{clll}
\hline ID & Threat & System Category & Threat to Goal \\
\hline T1 & Forge tickets & Live event & Confidentiality, integrity \\
T2 & Sneak inside & Live event & Confidentiality \\
T3 & Record content & Broadcast, Live event & Confidentiality, integrity \\
T4 & Broadcast content & Live event, Recording & Confidentiality, integrity \\
T5 & Steal recording & Recording & Confidentiality \\
T6 & Copy content & Recording & Confidentiality \\
T7 & Manufacture board & Game & Confidentiality, integrity \\
& game & & \\
\hline
\end{tabular}

Figure 9.6: Threats to physical rights management. 


\subsection{Properties of Physical Entities}

In this section, we try to account for the possibilities of threats by defining the properties of entities involved in the process (RQ1). First, Figure 9.7 shows the suport for the exising hypotheses. Second, Figure 9.8 shows the list of newly discovered properties and their impacts. In the next paragraphs, each property is described in more detail.

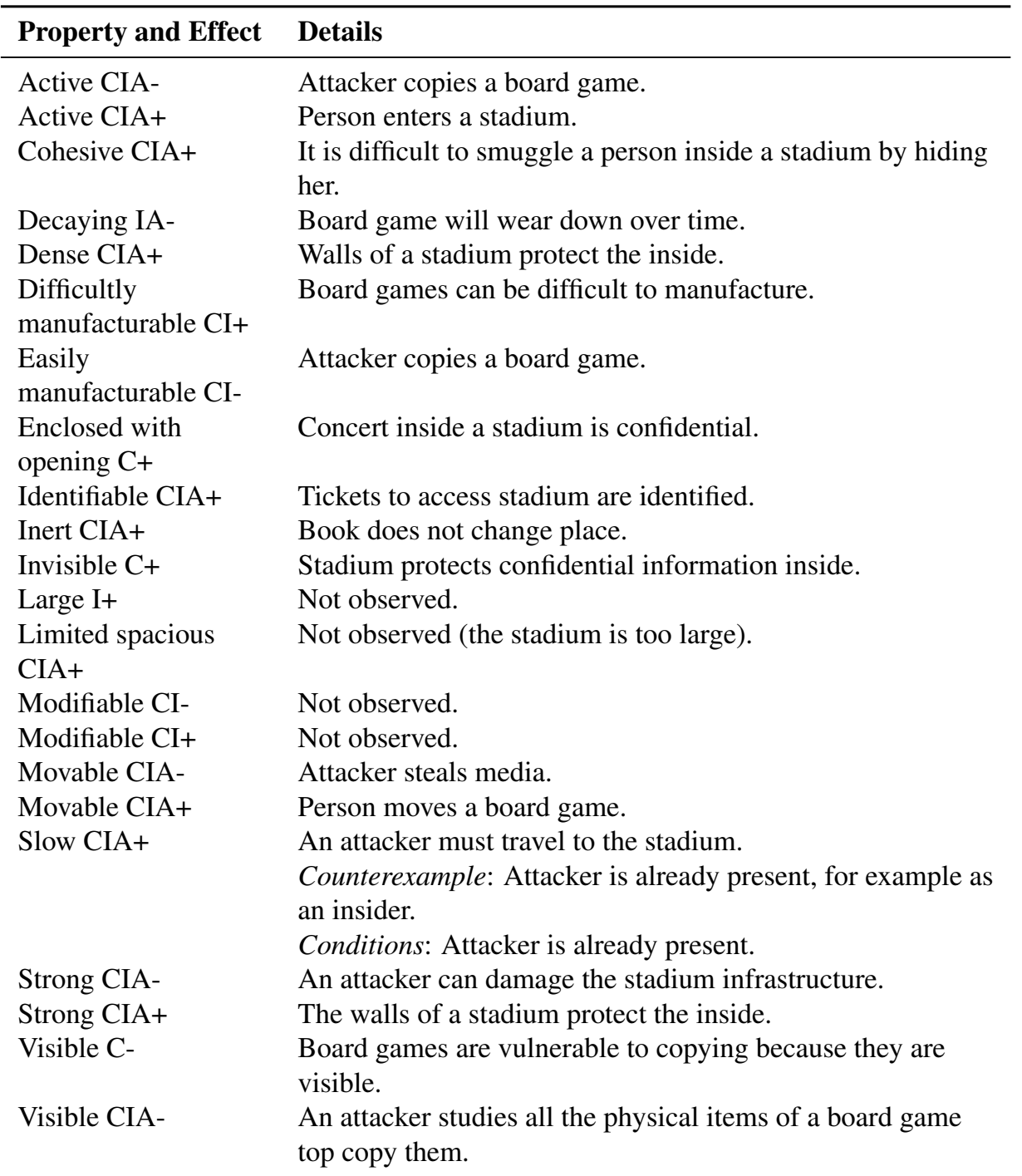




\begin{tabular}{ll}
\hline Property and Effect & Details \\
\hline Visible CIA+ & $\begin{array}{l}\text { Guards spot someone entering a stadium without } \\
\text { permission. }\end{array}$ \\
& $\begin{array}{l}\text { Conditions: There is a central policy enforcement point } \\
\text { such as a gate. }\end{array}$ \\
Weak CIA+ & Attacker cannot break through the wall of a stadium. \\
\hline
\end{tabular}

Figure 9.7: Support for existing hypotheses (consisting of properties and their effects) in the rights management case study. If counterexamples lead to new hypotheses they are listed individually in Figure 9.8 .

Decaying Wear diminishes the chances of distributing confidential information. An example specific to this case is that a physical medium such as a tape wears down when it is being played on a tape recorder. A counterexample is a physical object that is only used for a short time. This leads to one condition:

- The object is used long enough to experience wear.

Sound Producing An attacker can record sounds which limits their confidentiality. An example specific to this case study is that music can be recorded. A counterexample is that other sounds can be recorded but are not a threat to confidentiality. This leads to one condition:

- Sounds are confidential.

Uncopyable A person cannot be copied or manufactured which has a positive effect on confidentiality. An example specific to this case study is that a singer cannot be copied.

\begin{tabular}{lll}
\hline Property & Effect & Hypothesis \\
\hline Decaying & $\mathrm{C}+$ & $\begin{array}{l}\text { A decaying object contains only inaccurate confidential } \\
\text { information. }\end{array}$ \\
$\begin{array}{ll}\text { Sound producing } \\
\text { Uncopyable }\end{array}$ & $\mathrm{C}-$ & $\begin{array}{l}\text { An attacker records sounds. } \\
\text { Persons cannot be copied or manufactured. }\end{array}$ \\
\hline
\end{tabular}

Figure 9.8: New hypotheses from the rights management case study about the security effects of physical properties of entities. Each row represents either positive or negative effects. Multiple letters indicate an effect on multiple security goals. For example CIA- means a negative effect on confidentiality, integrity and availability. 


\subsection{Digital Rights Management}

Next, we collect data about digital systems to compare them to physical systems later (RQ2). Note that because we focus on digital systems, we exclude certain systems that also use physical mechanisms; these are described later in Section 9.10. Figure 9.9 shows the different entities for each digital case.

\begin{tabular}{llll}
\hline Entity Type & ID & Entity & Case (System) \\
\hline Content & D1 & Online video & Music (S17) \\
& D2 & PC game & Game (S11) \\
& D3 & PDF & Text (S2) \\
& D4 & MP3 & Music (S8) \\
& D5 & Live stream & Sports match (S16) \\
Medium & D6 & CD & (S11) \\
Player & D7 & PC & Music, Sports match (S7, S8, S11, S16, S17) \\
Recorder & D8 & CD writer & Game (S11) \\
Webserver & D9 & Media server & Music, Sports match (S7, S16, S17) \\
Consumer & D10 & User & (all) \\
\hline
\end{tabular}

Figure 9.9: Entities in digital rights management systems.

Figure 9.10 shows the operations. As in Section 9.5, the operations are divided between the operations performed by the producer and by the consumer. The second column indicates the generalized operation and is explained for each system in more detail.

\subsubsection{Threats and Mitigations}

We will now discuss several threats and mitigations concerning digital rights management. A first basic threat is to copy digital content (T1). CDs, PC games and MP3 files are all encoded as digital content and easily copied onto other media.

The Internet added a new threat to this, by making the distribution of content very easy (T2), such as the notorious case of the Napster website, where users could search for and download MP3 files [129]. A measure that is both preventive and detective is to add watermarks: "unremovable" information that is encoded into content, which can be used to identify the user that bought the content. The difficulty of removing watermarks (T3) is dependent on the scheme used [141].

Even if content copying cannot be prevented, it can still be possible to prevent its consumption. In the case of computer games, protection came from special software components that required the user to fill in license numbers. Only someone with a valid license number could use the software. The easiest attack is to reuse license numbers (T4). Another technique used for music and video is 


\begin{tabular}{|c|c|c|c|c|c|}
\hline ID & Operation & Recording & Game & Broadcast & Goals \\
\hline $\mathrm{P} 1$ & Create & $\begin{array}{l}\text { Create } \\
\text { recording }\end{array}$ & $\begin{array}{l}\text { Develop } \\
\text { game }\end{array}$ & $\begin{array}{l}\text { Create } \\
\text { content }\end{array}$ & G6 \\
\hline $\mathrm{P} 2$ & Protect & $\begin{array}{l}\text { Manufacture } \\
\text { CD }\end{array}$ & $\begin{array}{l}\text { Add } \\
\text { activation } \\
\text { component }\end{array}$ & $\begin{array}{l}\text { Encrypt } \\
\text { content }\end{array}$ & $\begin{array}{l}\text { G2, G6, } \\
\text { G8, G10 }\end{array}$ \\
\hline P3 & Distribute & Distribute CD & $\begin{array}{l}\text { Publish game } \\
\text { online }\end{array}$ & $\begin{array}{l}\text { Stream } \\
\text { content }\end{array}$ & $\mathrm{G} 3, \mathrm{G} 7$ \\
\hline P4 & $\begin{array}{l}\text { Monitor } \\
\text { usage }\end{array}$ & - & $\begin{array}{l}\text { Game } \\
\text { connects to } \\
\text { server }\end{array}$ & $\begin{array}{l}\text { Monitor } \\
\text { download } \\
\text { streams }\end{array}$ & $\begin{array}{l}\text { G10, G11, } \\
\text { G12 }\end{array}$ \\
\hline P5 & $\begin{array}{l}\text { Receive } \\
\text { payment }\end{array}$ & $\begin{array}{l}\text { Receive } \\
\text { payments }\end{array}$ & $\begin{array}{l}\text { Receive } \\
\text { payments }\end{array}$ & $\begin{array}{l}\text { Receive } \\
\text { payments }\end{array}$ & G1 \\
\hline $\mathrm{C} 1$ & Buy & $\begin{array}{l}\text { Buy } \\
\text { recording }\end{array}$ & $\begin{array}{l}\text { Buy game, } \\
\text { PC }\end{array}$ & $\begin{array}{l}\text { Buy stream, } \\
\text { PC }\end{array}$ & G4 \\
\hline $\mathrm{C} 2$ & Prepare & $\begin{array}{l}\text { Place } \\
\text { recording in } \\
\text { CD player }\end{array}$ & Install game & Open browser & G4 \\
\hline $\mathrm{C} 3$ & Consume & $\begin{array}{l}\text { Listen to } \\
\text { recording }\end{array}$ & Play game & Watch, listen & G4 \\
\hline
\end{tabular}

Figure 9.10: Operations of digital rights management systems and their contributions to goals.

encryption, such as DVDs using the CSS system, and play it using special software. An old approach used for the Commodore 64 home computer was to use non-documented ways of formatting disks [177].

However, it proved to be impossible to obfuscate the software to the extent that it could not be reverse engineered [12]. Through studying the runtime behavior of the software it is possible to understand its behavior and remove code that executes license number checks or to find the key that decrypts the media (T5). The latter happened with the CSS software for DVDs [183]) Furthermore, because the content such as music and video must be rendered to the user, the output can also be recorded from the computer's memory.

Web servers are a new defense mechanism. They can be first used for software activation: in order to use a license number the software must connect to the server of the producer, which can check if the software has been activated too many times. While this component can still be removed, it complicates the reverse engineering, as it makes it harder to study the code independently. Furthermore, in the case of music distribution, a site such as YouTube can perform fingerprinting on uploaded files, such that illegal files can be removed. A specific threat to live 


\begin{tabular}{clll}
\hline ID & Threat & System Category & Threat to Goal \\
\hline T1 & Copy content & Broadcast, & Confidentiality \\
& & Recording, Game & \\
T2 & Distribute content & Recording, Game & Control distribution \\
T3 & Remove watermarks & Recording & Limit piracy - detective, \\
& & & Limit piracy - deterrence \\
T4 & Reuse license numbers & Game & Limit piracy - preventive \\
T5 & Decrypt media & Recording & Limit piracy - preventive \\
T6 & Rebroadcast & Broadcast & Control distribution \\
\hline
\end{tabular}

Figure 9.11: Threats to digital rights management.

streams is rebroadcasting: once a user has payed for the content, it can be shared with other users (T6).

\subsection{Properties of Digital Entities}

Figure 9.12 shows the updates to the existing hypotheses from the earlier case studies. Most earlier hypothesis are supported and additional conditions have been added. Figure 9.13 lists the new hypotheses discovered in the IT infrastructure case.

\begin{tabular}{ll}
\hline Property and Effect & Details \\
\hline $\begin{array}{l}\text { Accurate Data Storage, } \\
\text { Transportation and Processing C- } \\
\text { Accurate Data Storage, } \\
\begin{array}{l}\text { Transportation and Processing } \\
\text { CIA+ }\end{array}\end{array}$ & $\begin{array}{l}\text { Data such as music can be transported accurately } \\
\text { which decreases confidentiality. } \\
\text { Digital media can be transmitted without failure. } \\
\text { Accurate Data Storage, } \\
\text { Transportation and Processing I- } \\
\text { Fast Data Processing A- }\end{array}$ \\
$\begin{array}{l}\text { Fast Data Processing C- } \\
\text { Fast Data Processing I- }\end{array}$ & $\begin{array}{l}\text { Not observed. } \\
\text { Not observed. } \\
\text { Not observed. } \\
\text { Counterexample: Monitoring can increase } \\
\text { integrity as well for remote systems (games, } \\
\text { always-on mode). } \\
\text { Easy storage on different locations increases } \\
\text { availability. }\end{array}$ \\
Fast Data Storage A+ & $\begin{array}{l}\text { Attacker can easily store data on other systems, } \\
\text { reducing confidentiality. }\end{array}$ \\
Fast Data Storage C- & Usage of digital material can be logged, if \\
centralized. \\
Conditions: The system is isolated.
\end{tabular}




\begin{tabular}{ll}
\hline Property and Effect & Details \\
\hline Fast Data Transportation A+ & $\begin{array}{l}\text { System transfers music files to other locations } \\
\text { over the Internet. } \\
\text { Someone transfers music files to other locations } \\
\text { over the Internet. } \\
\text { Fast Data Transportation C- }\end{array}$ \\
$\begin{array}{l}\text { Errors in rights management software are difficult } \\
\text { to prevent. }\end{array}$ \\
\hline
\end{tabular}

Figure 9.12: Support for existing hypotheses (consisting of properties and their effects) in the rights management case study. If counterexamples lead to new hypotheses they are listed individually in Figure 9.13.

Fast Data Processing Fast processing of data increases integrity because it makes it easier to monitor systems and detect errors and attacks. An example specific to this case study is that a central system keeps track of what's happening at remote locations and ensures integrity there. There are two conditions:

- There is a remote site.

- The system is distributed.

Program Computational Complexity Cryptographic functions help keep data confidential by encrypting data. Thus content is kept confidential and copying plain data is prevented. An example specific to this case study is that cryptography keeps music data confidential.

\begin{tabular}{lll}
\hline Property & Effect & Hypothesis \\
\hline Fast Data Processing & I+ & $\begin{array}{l}\text { System monitoring increases } \\
\text { integrity because it detects errors } \\
\text { and attacks. }\end{array}$ \\
$\begin{array}{l}\text { Program Computational } \\
\text { Complexity }\end{array}$ & CI + & $\begin{array}{l}\text { Cryptographic functions keep data } \\
\text { confidential. }\end{array}$ \\
\hline
\end{tabular}

Figure 9.13: New hypotheses from the rights management case study about the security effects of digital properties of entities. Each row represents either positive or negative effects. Multiple letters indicate an effect on multiple security goals. For example CIA- means a negative effect on confidentiality, integrity and availability. 


\subsection{Comparison between Physical and Digital Rights Man- agement}

We will now compare purely physical and digital rights management systems. Figure 9.14 lists the cases that are compared and the comparison between physical and digital properties is shown in Figure 9.15. From the figure it shows that in some cases we can easily compare physical and digital content management systems, such as a printed book versus a PDF. However in others a comparison is not possible: a live event is inherently physical and there is no digital substitute for being at a certain place. Broadcasting is possible, but this falls in a different category altogether as there are both digital and analog broadcasts. Furthermore the differences vary for each type of content: the physical - digital comparison for a text differs from that of a game.

\section{- Recordings}

Non-digital content (completely physical or analog) is not perfectly copyable. Content can be tied to a physical medium that must be copied along with the content. These mediums require space, have weight and deteriorate over time, such that they cannot be copied and/or transported easily. Furthermore, to make a copy, a user requires access to the medium first, which creates additional challenges such as that the user must be physically close to the medium. By contrast digital content allows for perfect copies that are transportable over a distance with little effort, via the Internet and consumed somewhere else. Thus it is much harder to enforce rights management for recordings in a digital way.

\section{- Broadcasts}

Analog broadcasts are less easy to record and distribute then digital broadcasts.

\begin{tabular}{lll}
\hline Content Type & Physical System & Digital System \\
\hline Text & S1. Book & S2. PDF \\
Music & S4. Live music & - \\
& S5. Tape recorder & S8. PC and MP3 player \\
& S6. Radio broadcast & S7. Internet radio \\
Game & S10. Board game & S11. PC game \\
Sports match & S13. Live match & - \\
& S14. Video recorder & S17. Video-sharing site \\
& S15. TV broadcast & S16. Live stream \\
\hline
\end{tabular}

Figure 9.14: Cases for comparison. 


\begin{tabular}{|c|c|c|c|}
\hline Security Goal & Effect & Physical Property & Digital Property \\
\hline All & + & $\begin{array}{l}\text { Active, Cohesive, Dense, } \\
\text { Identifiable, Inert, Movable, } \\
\text { Slow, Strong, Visible, Weak } \\
\text { Active, Movable, Strong, } \\
\text { Visible }\end{array}$ & $\begin{array}{l}\text { Accurate Data Storage, } \\
\text { Transportation and } \\
\text { Processing } \\
\text { Program Computational } \\
\text { Complexity }\end{array}$ \\
\hline \multirow[t]{2}{*}{ Confidentiality } & + & $\begin{array}{l}\text { Decaying, Difficultly } \\
\text { manufacturable, Enclosed } \\
\text { with opening, Invisible, } \\
\text { Uncopyable }\end{array}$ & $\begin{array}{l}\text { Program Computational } \\
\text { Complexity }\end{array}$ \\
\hline & - & $\begin{array}{l}\text { Easily manufacturable, } \\
\text { Sound producing, Visible }\end{array}$ & $\begin{array}{l}\text { Accurate Data Storage, } \\
\text { Transportation and } \\
\text { Processing, Fast Data } \\
\text { Storage, Fast Data } \\
\text { Transportation }\end{array}$ \\
\hline \multirow[t]{2}{*}{ Integrity } & + & Difficultly manufacturable & $\begin{array}{l}\text { Fast Data Processing, Fast } \\
\text { Data Storage, Program } \\
\text { Computational Complexity }\end{array}$ \\
\hline & - & $\begin{array}{l}\text { Decaying, Easily } \\
\text { manufacturable }\end{array}$ & N/A \\
\hline \multirow[t]{2}{*}{ Availability } & + & N/A & $\begin{array}{l}\text { Fast Data Storage, Fast Data } \\
\text { Transportation }\end{array}$ \\
\hline & - & Decaying & N/A \\
\hline
\end{tabular}

Figure 9.15: Comparison of physical and digital properties on their ability to realize security goals for the rights management case study. This table is constructed from data presented in Figures 9.7, 9.8, 9.12 and 9.13. 


\begin{tabular}{lll}
\hline Goals & Physical Rights Management & Digital Rights Management \\
\hline Confidentiality & + content requires & - content requires no \\
& manufacturing effort & manufacturing effort \\
& + content requires distribution & - content is non-local \\
& effort & + can monitor usage remotely \\
& + content is place and time & \\
& bound & \\
& + can monitor usage locally & \\
Distribution & - requires distribution effort & + requires no distribution effort \\
\hline
\end{tabular}

Figure 9.16: Comparison between physical and digital rights management system. "+" and "-" indicate positive and negative effects, whereas \pm indicates a mixed effect. The explanation of the scores for each goal and system is given in Section 9.9

\section{- Games}

The comparison for games yields a classical comparison between physical and digital goods: physical games require manufacturing equipment and raw materials to copy. By contrast, copying a PC game only requires a computer and storage space. As for transportation of physical games, this requires time and money, whereas distribution over the Internet is almost instantaneous and free.

\section{- Live events}

As noted before, live events are a separate category that do not have a clear digital equivalent. The manufacture, distribution and consumption all take place at the same physical location. This location is physically secured, and we can control who receives access to it. Usage is also instantaneous, we cannot store an orchestra to use for a later time. A sports match cannot be copied, in the sense that the players and the stadium themselves cannot be copied. Recording a live event is possible, but this requires equipment, which is detectable.

We will now show the aggregated differences of all cases in Figure 9.16, and then explain the differences for each category. In the physical world, two basic mechanisms help to ensure confidentiality: the difficulty to manufacture goods and the difficulty of distributing them. For live events - it is also their place and time-boundedness: persons have to be present at a location to consume the content. While they are at that location, or try to enter, they can be monitored to ensure that only authorized persons have access to the content. By contrast, digital manufacturing and distribution require little effort, and this makes it easy to consume them remotely, which limits confidentiality. However, digital systems 
have the option to monitor consumers remotely, and control confidentiality in this way. Regarding distribution, the distribution effort for physical and digital content is clearly opposite.

\subsection{Hybrid Rights Management}

In all our case studies we investigated the possibilities and strengths of hybrid rights management. As discussed in Section 9.2, hybrid systems concern systems that use a combination of physical and digital enforcement techniques to secure content. In this section we are especially interested in protection for digital content.

Figure 9.17 shows the entities used in hybrid rights management systems. Operations are listed in Figure 9.18. The operations are divided between the operations performed by the producer and by the consumer. The producer's operations consist of a pattern with four main operations: create content, manufacture content, distribute content and receive payments. The consumer's pattern is to buy content, ready it for usage and finally consume it.

\begin{tabular}{llll}
\hline Entity Type & ID & Entity & Case (System) \\
\hline File & E1 & E-book & Text (S3) \\
& E2 & Encrypted Music file & Music (S9) \\
& E3 & Game & Game (S12) \\
Broadcast & E4 & Encrypted live stream & Sports match (18) \\
Player (content) & E5 & E-reader (Kindle) & Text (S3) \\
& E6 & Portable music player & Music (S9) \\
& E7 & Game Console & Game (S12) \\
& E8 & TV and Set-top box & Sports match (S18) \\
Server & E9 & Digital music store & Music (S9) \\
& E10 & Digital book store & Text (S3) \\
& E11 & Computer game server & Game (S12) \\
& E12 & Live stream server & Sports match (S18) \\
& E13 & Consumer & \\
\hline \multirow{2}{*}{ Consumer } & & & \\
\hline
\end{tabular}

Figure 9.17: Entities in hybrid rights management systems.

\subsubsection{Threats and Mitigations}

In contrast to the earlier sections we begin with the mitigations and then explain threats to these. In total we see four mitigation strategies (Figure 9.19) that link digital content tighter with physical phenomena. 


\begin{tabular}{|c|c|c|c|c|c|}
\hline ID & Operation & Recording & Online Game & Pay-per-view & Goals \\
\hline P1 & Create & Create recording & Develop game & Play match & G6 \\
\hline $\mathrm{P} 2$ & Protect & $\begin{array}{l}\text { Encrypt contents, } \\
\text { protect keys }\end{array}$ & $\begin{array}{l}\text { On-line } \\
\text { activation }\end{array}$ & $\begin{array}{l}\text { Encrypt } \\
\text { contents, } \\
\text { protect keys }\end{array}$ & $\begin{array}{l}\text { G2,G6, } \\
\text { G8,G10 }\end{array}$ \\
\hline P3 & Distribute & $\begin{array}{l}\text { Sell player and } \\
\text { content }\end{array}$ & Publish online & Stream & G3,G7 \\
\hline P4 & $\begin{array}{l}\text { Monitor } \\
\text { usage }\end{array}$ & Connect to server & Connect to server & - & $\begin{array}{l}\text { G10,G11, } \\
\text { G12 }\end{array}$ \\
\hline P5 & $\begin{array}{l}\text { Receive } \\
\text { payment }\end{array}$ & $\begin{array}{l}\text { Receive } \\
\text { payments }\end{array}$ & $\begin{array}{l}\text { Receive } \\
\text { payments }\end{array}$ & $\begin{array}{l}\text { Receive } \\
\text { payments }\end{array}$ & G1 \\
\hline $\mathrm{C} 1$ & Buy & $\begin{array}{l}\text { Buy player and } \\
\text { content }\end{array}$ & $\begin{array}{l}\text { Buy game, } \\
\text { console }\end{array}$ & $\begin{array}{l}\text { Buy set-top } \\
\text { box, keys }\end{array}$ & G4 \\
\hline $\mathrm{C} 2$ & Prepare & $\begin{array}{l}\text { Download } \\
\text { content }\end{array}$ & Install game & Install codes & G4 \\
\hline $\mathrm{C} 3$ & Consume & $\begin{array}{l}\text { Listen, read } \\
\text { recording }\end{array}$ & Play game & Watch & G4 \\
\hline
\end{tabular}

Figure 9.18: Operations of hybrid rights management systems and their contributions to goals.

\begin{tabular}{llll}
\hline ID & Threat & System Category & Goal \\
\hline M1 & Explicit Grounding to End-user Devices & All & Confidentiality \\
M2 & Implicit Grounding to End-user Devices & All &, \\
M3 & Grounding to a Centralized Server & All &, \\
M4 & Grounding to Individuals & Live event, Game &, \\
\hline
\end{tabular}

Figure 9.19: Specific mitigations for hybrid rights management.

Explicit Grounding to End-user Devices The first mitigation strategy M1 is to explicitly tie content to particular end-user devices. Traditionally, this means using a specific device meant for copyright purposes, such as a dongle that must be present on the PC running a software program, or a set-top box that is connected to a tv. These devices can perform simple computations and store data. In the case of dongles, the software running on the PC can then check for the presence of the dongle (with attacks against code obfuscation as described before in Section 9.7). Another option is to physically tie encryption and decryption keys to the hardware, such that the system is harder to crack. This strategy can be applied to all types of content. Sometimes however, these chips can be removed, so called "modding", in other cases it turns out to be possible to recover the keys. One particular problem that content producers face is that the device is physically accessible to the enduser, and thus relatively easy to modify or reverse engineer. 
Implicit Grounding to End-user Devices The second mitigation strategy M2 is not to use explicit but rather implicit mechanisms to tie content to hardware. These implicit mechanisms assure that, although attacks remain technically possible, the consumer can only easily use the content on a particular device, and is thus inclined to accept the restrictions. Once the content is tied to the device, other strategies (including digital mechanisms) can be used to secure the content further. There are three main ways to realize implicit grounding:

\section{- Required Data Processing Power}

Content can be created such that it cannot be executed unless specific computing power is available. For example, computer games cannot be played unless a high performance graphical chip is present. This property ties the content closer to specific hardware.

- Required Data Storage

Content that requires a lot of data storage is harder to copy. This is particularly true for high resolution content such as used in movies. A hard drive can hold tens of thousands of songs, but storing tens of thousands of movies is much more difficult. One reason why music is hard to secure is that the files are relatively small and it is not possible to increase their size in a meaningful way, for example by offering superior sound quality.

\section{- Required Data Visualization or Interactivity}

Content can be created and optimized for a specific display mechanism (such as an iPhone screen). It can technically be used on another device, but the content is less enjoyable because of conversion problems such as different screen sizes.

Interactive content requires active participation from the user, such as in a video game that reacts to the user. For example, the Nintendo Wii $4^{4}$ has a controller equipped with a movement sensor that the user must use. Copying a game is not useful unless the user also copies the controller (has the manufacturing capabilities to do this) or already has a similar (likely expensive) controller. By contrast, listening to music does not require such interaction.

Grounding to a Centralized Server A third mitigation strategy is to use centralized servers (M3). This strategy can be used for computer games, which must connect to these servers in order to run properly. It is difficult to circumvent the usage control of such games, because we cannot reverse engineer and recreated it; Several physical mechanisms are at work to prevent this:

\footnotetext{
$\sqrt[4]{\text { http://www.nintendo.com/wii }}$
} 
first, a server used in a game is physically remote from the users, and thus inaccessible, except through the Internet. Thus, not all software is available for copying as the central server connecting all users is not public. This makes it more difficult to steal, modify, copy or reverse engineer.

Second, the bandwidth and computing requirements can be large if the game is played by hundreds of thousands of users. In spite of gains in network speed. Internet connections still have a limited physical bandwidth and this can act as a natural DRM [20].

Grounding to Individuals The fourth mitigation strategy M4 is to ground content usage to users. Even if all the content and necessary hardware can be copied, users remain physically uncopyable and their behavior can be difficult to influence. This strategy works when content is used by thousands of other people over the Internet using a central server. If a user decides to setup her own networked game, she still has to contact and convince all these users to shift from the original server to a pirated one. This can be so cumbersome that piracy is ineffective. This strategy requires of course that the content is shared between users - which is the case for games, but less so for music and text.

\subsubsection{Types of Hybrid Systems}

The previous section listed four ways to ground content to physical phenomena, and we can use these to build four types of hybrid systems $(\mathbf{Q 3})$. They can be dependent on

- IT resources (implicitly): we can design content (especially games) that requires special data processing power, storage, visualization or interactivity, and thus can only be played on certain devices.

- IT resources (explicitly): often in combination with the latter, physical hardware can be used to store cryptographic keys securely and prevent piracy.

- Centralized server: a server accessible via the Internet grounds in three ways:

- As it is in a physically remote location, it cannot easily be stolen.

- The bandwidth and server resources needed for its operation are difficult and/or costly to duplicate.

- The consumers using the server (and interacting with other users) are uncopyable.

- Persons: we can design content (especially games) such that they need many users that cannot shift to pirated versions easily. 


\begin{tabular}{|c|c|c|}
\hline \# & Explanation & Example \\
\hline S07 & $\begin{array}{l}\text { Implicit dependent on data } \\
\text { processing }\end{array}$ & CPU power \\
\hline S08 & Implicit dependent on data storage & Large data storage \\
\hline S09 & $\begin{array}{l}\text { Implicit dependent on data } \\
\text { visualization }\end{array}$ & Specific visualizations \\
\hline S10 & $\begin{array}{l}\text { Implicit dependent on data } \\
\text { interactivity }\end{array}$ & Specific user interactions \\
\hline S11 & Explicit dependent on hardware & Specific hardware or network \\
\hline S12 & Person dependent & Person \\
\hline
\end{tabular}

Figure 9.20: Types of hybrid systems in the rights management case study.

\subsection{Combinations and Trade-off Analysis}

\subsubsection{Trade-off Analysis on Properties}

Figure 9.21 and 9.22 show the trade-off analysis on the effect of physical and digital properties. Regarding physical properties the results are mixed, with four properties having no significant effect on either physical or hybrid systems. As for digital properties, the hybrid systems can cancel out their negative effects.

\subsubsection{Trade-off Analysis on Goals}

Figure 9.23 shows the trade-off analysis done for the individual cases, specific for confidentiality, as this is the main security goal for rights management. The results are explained in the next paragraphs.

First, compared to physical solutions, our analysis indicates that the results depend on the type of content to be protected. For text and music, hybrid systems are inferior in terms of confidentiality, because content to the physical part of a hybrid system is challenging, as discussed in the previous section. Hybrid systems that serve games and sports matches are better at securing content, because they can tie it more easily to a physical resource, or monitor user behavior.

Regarding the comparison of hybrid to digital systems: hybrid systems do offer advantages over them, again because they can make use of the hybrid security mechanisms outlined before. The exception is music: no known mechanism exists that ties music to a hybrid system. 


\begin{tabular}{lllll}
\hline & & & \\
& & &
\end{tabular}

Figure 9.21: Trade-off analysis on the impact of properties between hybrid and physical for the rights management case study. Positive scores indicate that the hybrid system is more secure than the physical system, negative scores that it is not. N/A means that the effect was not observed in the case study. (For the exact operationalization details, see Figure 5.11 in Chapter 5) The full explanations of the scores for each goal are Section 9.11 . 


\begin{tabular}{llll}
\hline & & & \\
Hypothesis & & &
\end{tabular}

Figure 9.22: Trade-off analysis on the impact of properties between hybrid and digital for the rights management case study. Positive scores indicate that the hybrid system is more secure than the digital system, negative scores that it is not. N/A means that the effect was not observed in the case study. (For the exact operationalization details, see Figure 5.11 in Chapter 5.) The full explanations of the scores for each goal are Section 9.11 . 


\begin{tabular}{|c|c|c|c|c|}
\hline System Type & Text & Music & Game & Sports Match \\
\hline Hybrid & $\begin{array}{l}\text { E-reader and } \\
\text { E-book (S3) }\end{array}$ & $\begin{array}{l}\text { Portable media } \\
\text { player (S9) }\end{array}$ & $\begin{array}{l}\text { Online console } \\
\text { game (S12) }\end{array}$ & $\begin{array}{l}\text { Pay-per-view } \\
\text { (S18) }\end{array}$ \\
\hline Physical & Book (S1) & $\begin{array}{l}\text { Tape recorder } \\
\text { (S5) }\end{array}$ & $\begin{array}{l}\text { Board game } \\
\text { (S10) }\end{array}$ & $\begin{array}{l}\text { TV broadcast } \\
\text { (S15) }\end{array}$ \\
\hline Digital & $\mathrm{PDF}(\mathrm{S} 2)$ & $\begin{array}{l}\mathrm{PC} \text { and MP3 } \\
\text { player (S8) }\end{array}$ & PC game (S11) & $\begin{array}{l}\text { Live stream } \\
\text { (S16) }\end{array}$ \\
\hline $\begin{array}{l}\text { Compared to } \\
\text { physical }\end{array}$ & - & - & + & + \\
\hline $\begin{array}{l}\text { Compared to } \\
\text { digital }\end{array}$ & + & $=$ & + & + \\
\hline
\end{tabular}

Figure 9.23: Comparison of hybrid with physical and digital rights management systems on confidentiality. (For the exact operationalization details, see Figure 5.11 in Chapter 5) The explanation of the scores for each goal is given in Section 9.11

\subsection{Conclusion}

In the last section, we summarize the answers on our general research questions, and also attempt to answer $\mathbf{R Q} \mathbf{4}_{\mathrm{RM}}$ about the possibility of building a working RM system for music.

First, concerning (RQ1) about physical properties: we conclude that the effectiveness of physical rights management heavily depends on the properties of the physical medium. First, physical media require space, have weight and deteriorate over time, such that they cannot be copied and/or transported easily. Furthermore, to make a copy, a user requires access to the medium first, which creates additional challenges such as that the user must be physically close to the medium. By contract, an analog broadcast cannot be easily grounded to a physical phenomenon. In other cases, visibility also plays a role, for example in the context of live events, where consumers have to pay at the entrance.

Live events are very effective in enforcing confidentiality. To begin, they are dependent on time. Static content, such as a board game does not change form and it therefore relatively easy to copy and the copy has a more lasting value. By contrast, content that is of a perishable nature such as a live football match diminishes in value quickly after its creation. Furthermore, they are tied to a particular location where it can be consumed. Usage control is possible due to the spatial architecture, which limits the observation of the performance by persons, who need to enter through a specific entrance. Because of their size and visibility, persons must pay at this entrance, and this secures the revenue stream. As for 
copying, performances can be recorded but they cannot be recreated because the persons doing the performance cannot be recreated.

Second, the comparison of physical and digital rights management ( $\mathbf{Q 2})$ shows the main differences to be that physical systems are secure because of local interaction, whereas digital systems can be monitored remotely. Monitoring can be used by some forms of content (live music events) but makes much less sense for books, which persons read without interacting. A well-known weakness of digital systems compared to physical ones is that the distribution and manufacture of digital content is much easier.

We attempted to find different ways of constructing hybrid systems $(\mathbf{Q 3})$. We found four main types of hybrid rights management systems: which tie content to IT resources (either implicitly or explicitly), to centralized servers or to individuals.

Fourth, the trade-off analysis (RQ4) indicates that the effectiveness of solutions depends on the particular content type, but that hybrid solutions are generally better than digital ones.

\subsubsection{Possibility of a Working RM System for Music}

Our findings challenge the existing problem definitions concerning DRM. In DRM research, the objectives are to find adequate and generic protection mechanisms for existing digital content. Mechanisms such as secure containers and watermarking can then be implemented into any digital content. Another problem definition that has emerged is that DRM is fundamentally flawed because copying digital content cannot be prevented, any more than water can be made dry. Indeed, in our research, we have not encountered any system used by consumers that actually offered a truly secure container. All containers that are in use today can be breached with some effort after some time. Both problem definitions are inadequate for understanding the challenges of DRM.

The activity to improve RM systems is best understood not as a quest for adequate protection mechanisms for existing content, as this content will always be easier to copy in the future. Rather, it is a search for new content types that are hard to copy. (Or in Benjamin's terms, a search for new types of art.) This search is not futile, as the existence of online games demonstrates. We are far from locked in a trend of increasing copyability.

Effective rights management always requires a complicated and expensive physical manufacturing process to create, copy, distribute and/or use it. Content that requires specific capabilities of hardware such as processing power, storage or communication has a natural form of DRM. Even without any copy protection, the content requires specific hardware. An example is a computer game that required a high performance graphical processing unit. Once the content is tied to hardware, the hardware can be used for further security, but this is not the 
main copyright protection mechanism. Interactivity with persons further improves copyright protection.

Our results thus offer a simple explanation for the failure to protect digital music. The reason that music is easy to copy is not because of lack of secure containers, rather it is a consequence of the content type of music itself. Regarding all content protection mechanisms, music scores low in terms of content protection: It is not physical, not time-bounded, not location-dependent and not interactive. Concerning computing capabilities, it requires little storage, processing, communication and neither is visualization required: general purpose hardware has sufficed for the past decades. This view also explains why devices such as the Amazon Kindle are profitable (at least for today): as the hardware to display E-books is not widely available in other devices, it grounds the E-books to the Kindle. New types of content are easier to protect and they indeed utilize more physical protection mechanisms. Internet games playable via a physical console are a good example of this.

This concludes the last case study of Part III In the next part we will summarize and discuss the results from all of the case studies. 


\section{Part III}

\section{Summary, Application and Validation}





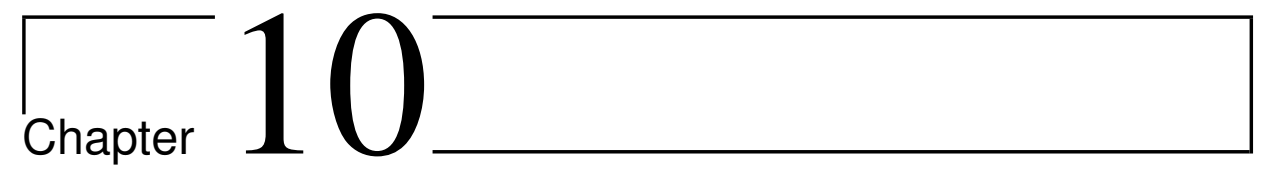

\section{Conceptual Model of Integrated Physical and Digital Security}

\subsection{Introduction}

This thesis began with the objective of delivering results to help professionals and researchers improve information security in a de-perimeterized world. To this end, we examined different physical and digital security mechanisms and combinations thereof. This chapter integrates the results from the case studies into one conceptual model and answers the research questions RQ1-RQ5.

We will do this in the similar approach that we followed in the previous sections, taking all the results from these case studies into account. We list all the properties of physical entities that were found in the four case studies in Section 10.3. This answers question RQ1: What are the properties of physical entities that physical security mechanisms depend upon? Next Section 10.4 lists the digital properties. This answers question RQ2: What are the properties of digital entities that digital security mechanisms depend upon? We then compare physical and digital security mechanisms in Section 10.5. This answers question RQ3. What are the key differences between physical and digital security mechanisms? Next, we categorize different types of combinations of physical and digital security mechanisms, answering question RQ4. How can physical and digital security mechanisms be combined? Following this, we assess how hybrid solutions can improve security in Section 10.6, answering question RQ5. What are the tradeoffs between physical and digital mechanisms? Finally we discuss the validity of the results in Section 10.8.

But first the methods for analyzing the four case studies are presented. 


\subsection{Method}

As stated in Chapter 5 the main method to process the case study results is analytical induction: analyzing the results step by step and building up knowledge with each new case [180]. As such, this chapter simply presents a summary, specifically for the main results which are the hypothesis about the impact of digital and physical properties (RQ1 and RQ2). However, for the other research questions about differences, combinations and trade-offs, we do analyze the data further to find more meaning in them. For this we followed the method presented by Eisenhardt [53]. The idea behind cross-case study analysis is that by constantly comparing the researcher's preliminary theories with data it is possible to construct theories that are tightly linked to that data. This tight integration should result in conclusions with support in the data. (Note that this does not give 100\% certainty - to provide more support, more research is always possible.) No data should be thrown away just because it does not suit a preliminary theory. Rather, the data should be studied in various ways: cases should be compared to find similarities and differences between them. For similar cases, we examine differences, whereas for different cases we attempt to discover similarities. From this analysis arise categories, concepts and constructs, which after a series of iterations are further refined. The cross-examination of data is split across the earlier division that we made: first we construct a coherent set of properties of physical and digital properties from all of our case studies. Second, we compare physical systems with other physical systems, and digital with digital systems. Third, we compare hybrid systems with physical and digital systems.

\subsection{Properties of Physical Entities}

Figure 10.1 lists all the physical properties that we have found and their impact on security, together with the conditions under which these effects hold true.

\begin{tabular}{|c|c|c|c|}
\hline Property & Effect & Hypothesis & Conditions \\
\hline Active & CIA- & An attacker disrupts a process. & The person is not malicious. \\
\hline Active & CIA+ & $\begin{array}{l}\text { A person intervenes in a } \\
\text { process to increase its security. }\end{array}$ & The person is malicious. \\
\hline Cohesive & $\mathrm{CIA}+$ & $\begin{array}{l}\text { An attacker cannot use a } \\
\text { cohesive object to hide other } \\
\text { objects inside it. }\end{array}$ & $\begin{array}{l}\text { The process is observed by a } \\
\text { defender. }\end{array}$ \\
\hline
\end{tabular}




\begin{tabular}{|c|c|c|c|}
\hline Property & Effect & Hypothesis & Conditions \\
\hline Decaying & $\mathrm{C}+$ & $\begin{array}{l}\text { A decaying object contains } \\
\text { only inaccurate confidential } \\
\text { information. }\end{array}$ & $\begin{array}{l}\text { The object contains confidential } \\
\text { information. } \\
\text { The object is used long enough } \\
\text { to experience wear. } \\
\text { The object should not act as a } \\
\text { capability itself. }\end{array}$ \\
\hline Decaying & IA- & $\begin{array}{l}\text { A decaying object becomes } \\
\text { damaged and unusable. }\end{array}$ & Objects are frequently used. \\
\hline Dense & $\mathrm{CIA}+$ & $\begin{array}{l}\text { A dense physical object acts as } \\
\text { a barrier for persons who } \\
\text { cannot get inside. }\end{array}$ & $\begin{array}{l}\text { The attacker has no alternative } \\
\text { means to obtain access. }\end{array}$ \\
\hline $\begin{array}{l}\text { Difficultly } \\
\text { manufac- } \\
\text { turable }\end{array}$ & $\mathrm{CI}+$ & $\begin{array}{l}\text { A difficultly manufacturable } \\
\text { object is hard to create or alter } \\
\text { precisely. }\end{array}$ & $\begin{array}{l}\text { Business process depends on } \\
\text { limited and/or unique items. }\end{array}$ \\
\hline $\begin{array}{l}\text { Easily manu- } \\
\text { facturable }\end{array}$ & CI- & $\begin{array}{l}\text { If an object is easy to } \\
\text { manufacture this reduces its } \\
\text { uniqueness and threatens any } \\
\text { process that depends on it. }\end{array}$ & $\begin{array}{l}\text { The attacker has the capability } \\
\text { to reproduce the object. } \\
\text { The objects act as a capability } \\
\text { to access something. } \\
\text { The attacker has the } \\
\text { opportunity to use the object in } \\
\text { the process. }\end{array}$ \\
\hline $\begin{array}{l}\text { Enclosed with } \\
\text { opening }\end{array}$ & $\mathrm{C}+$ & $\begin{array}{l}\text { An enclosure with opening } \\
\text { allows unobserved access to the } \\
\text { confidential information stored } \\
\text { inside. }\end{array}$ & $\begin{array}{l}\text { Person is not malicious. } \\
\text { Persons are screened for } \\
\text { reliability. }\end{array}$ \\
\hline Identifiable & $\mathrm{CIA}+$ & $\begin{array}{l}\text { A defender identifies objects to } \\
\text { understand the security status } \\
\text { of the process. }\end{array}$ & \\
\hline Inert & $\mathrm{CIA}+$ & $\begin{array}{l}\text { An inert object cannot alter or } \\
\text { expose itself or make itself } \\
\text { unusable. }\end{array}$ & $\begin{array}{l}\text { There is no wear. } \\
\text { The object does not have } \\
\text { autonomous capabilities. }\end{array}$ \\
\hline Invisible & $\mathrm{C}+$ & $\begin{array}{l}\text { Invisible information cannot be } \\
\text { stolen without attacking the } \\
\text { object that contains it. }\end{array}$ & $\begin{array}{l}\text { Access is physically restricted. } \\
\text { No confidential information is } \\
\text { stored. } \\
\text { There is a physical object that } \\
\text { contains confidential } \\
\text { information. }\end{array}$ \\
\hline Large & $\mathrm{I}+$ & $\begin{array}{l}\text { An attacker cannot hide a large } \\
\text { object. }\end{array}$ & $\begin{array}{l}\text { The object is used in the } \\
\text { process. } \\
\text { The process depends on unique } \\
\text { objects. }\end{array}$ \\
\hline $\begin{array}{l}\text { Limited } \\
\text { spacious }\end{array}$ & $\mathrm{CIA}+$ & $\begin{array}{l}\text { The small size of a location } \\
\text { reduces the opportunities for an } \\
\text { attacker to hide herself. }\end{array}$ & $\begin{array}{l}\text { The location in which a process } \\
\text { takes place is small in size. }\end{array}$ \\
\hline
\end{tabular}




\begin{tabular}{|c|c|c|c|}
\hline Property & Effect & Hypothesis & Conditions \\
\hline Modifiable & CI- & $\begin{array}{l}\text { An attacker modifies entities } \\
\text { and changes their state. }\end{array}$ & $\begin{array}{l}\text { The attacker is able to modify } \\
\text { objects unobserved. } \\
\text { The attacker precisely modifies } \\
\text { objects. }\end{array}$ \\
\hline Modifiable & $\mathrm{CI}+$ & $\begin{array}{l}\text { Persons modify entities as part } \\
\text { of the normal business } \\
\text { processes. }\end{array}$ & $\begin{array}{l}\text { Physical objects are modified in } \\
\text { the process. }\end{array}$ \\
\hline Movable & CIA- & $\begin{array}{l}\text { An attacker disrupts a process } \\
\text { by moving objects. }\end{array}$ & \\
\hline Movable & $\mathrm{CIA}+$ & $\begin{array}{l}\text { Movement of persons and } \\
\text { objects is necessary for } \\
\text { executing a process. }\end{array}$ & The person is not malicious. \\
\hline Slow & $\mathrm{CIA}+$ & $\begin{array}{l}\text { Slow objects remain close to } \\
\text { their intended location, } \\
\text { requiring an attacker to travel. }\end{array}$ & $\begin{array}{l}\text { Attacker is already present. } \\
\text { There are no insiders. } \\
\text { The attacker is external and } \\
\text { located somewhere else. }\end{array}$ \\
\hline $\begin{array}{l}\text { Sound } \\
\text { producing }\end{array}$ & $\mathrm{C}-$ & An attacker records sounds. & Sounds are confidential. \\
\hline Strong & CIA- & $\begin{array}{l}\text { A strong physical object is } \\
\text { useful to break a security } \\
\text { mechanism. }\end{array}$ & $\begin{array}{l}\text { The security mechanism is } \\
\text { physically weak. } \\
\text { There is no monitoring in place. }\end{array}$ \\
\hline Strong & $\mathrm{CIA}+$ & $\begin{array}{l}\text { A strong physical object } \\
\text { protects confidential } \\
\text { information. }\end{array}$ & $\begin{array}{l}\text { A physical object contains } \\
\text { confidential information. } \\
\text { The physical protection is } \\
\text { strong enough to withstand } \\
\text { attack. } \\
\text { Defenders observe the physical } \\
\text { object. } \\
\text { The attacker does not have the } \\
\text { capability to retrieve the } \\
\text { confidential information. }\end{array}$ \\
\hline Uncopyable & $\mathrm{C}+$ & $\begin{array}{l}\text { Persons cannot be copied or } \\
\text { manufactured. }\end{array}$ & \\
\hline Visible & $\mathrm{C}-$ & $\begin{array}{l}\text { An attacker obtains information } \\
\text { when it is visible to her. }\end{array}$ & $\begin{array}{l}\text { There is a physical object that } \\
\text { displays confidential } \\
\text { information. } \\
\text { The attacker should have the } \\
\text { means to copy or remember the } \\
\text { data: for example a physical } \\
\text { key must be copied or } \\
\text { photographed with a camera. }\end{array}$ \\
\hline
\end{tabular}




\begin{tabular}{|c|c|c|c|}
\hline Property & Effect & Hypothesis & Conditions \\
\hline Visible & CIA- & $\begin{array}{l}\text { An attacker observes a system } \\
\text { and uses this information to } \\
\text { attack it. }\end{array}$ & $\begin{array}{l}\text { An attacker observes the } \\
\text { process. } \\
\text { An attacker exploits } \\
\text { weaknesses in the security } \\
\text { mechanisms. }\end{array}$ \\
\hline Visible & CIA+ & $\begin{array}{l}\text { An object's visibility allows a } \\
\text { defender to deter, detect and } \\
\text { respond to threats. }\end{array}$ & $\begin{array}{l}\text { Defenders understand the } \\
\text { security effects of (attacker) } \\
\text { actions. } \\
\text { Officials must pay attention. } \\
\text { The physical object does not } \\
\text { expose confidential information } \\
\text { itself. } \\
\text { There is a central policy } \\
\text { enforcement point such as a } \\
\text { gate. }\end{array}$ \\
\hline Weak & CIA+ & $\begin{array}{l}\text { An attacker cannot bypass } \\
\text { security mechanisms because } \\
\text { she lacks physical strength. }\end{array}$ & Physical barrier is present. \\
\hline
\end{tabular}

Figure 10.1: Complete list of physical properties and their effects.

With these results, we performed a careful study of all properties found in the case studies. Analyzing their differences and similarities led to the identification of different clusters of properties (Figure 10.2). Each cluster represents a related set of properties that are relevant for security.

- The architectural cluster shows that spaces plays a great role in providing security. Physical entities such as servers are secure because they are locked inside a data center. A ballot box does not provide enough space to hold thousands of ballots, and this limits the options for voting fraud. The opening of the ballot box only allows the deposit of at most one or two ballots at a time. Thus architecture provides assurance of the confidentiality, availability and integrity of information.

- Audible entities impact security because the sounds they produce might be confidential. Apart from the case study on rights management it was not encountered in other case studies as having a specific effect.

- Independence concerns the ability of entities to act or to resist action from others. Physical structures are resistant to change and do not act: persons cannot walk through walls, paper does not monitor the writer, keys do not alter shape, and persons need physical force to break open a lock. Inertness provides assurance that a system configured in a particular way will be the 


\begin{tabular}{ll}
\hline Cluster & Property \\
\hline Architectural & Dense \\
& Enclosed with opening \\
& Limited spacious \\
Audible & Sound producing \\
Independence & Active \\
& Inert \\
& Movable \\
& Slow \\
Material & Cohesive \\
& Decaying \\
& Difficultly manufacturable \\
& Easily manufacturable \\
& Modifiable \\
& Strong \\
& Uncopyable \\
& Weak \\
& Identifiable \\
& Invisible \\
& Large \\
& Visible \\
\hline
\end{tabular}

Figure 10.2: Clusters of physical properties.

same in the next moment, even if no one is observing it. Our analysis indicates that inertness has two distinct tasks in promoting security.

First, physical security mechanisms are often inert themselves - walls, locks, ballot boxes are in a low energy state and cannot change themselves and they resist change from outside. These physical security mechanisms protect a space.

Second, their inertness also provides assurance of the inertness of the space inside it and the entities that must be protected. As an example: because the ballot box is inert and the ballots are inert, we are sure that the votes remain the same. The same holds true for a data center: because of physical security, we are certain that the inert cables do not change position. By contrast, in an open environment inertness does not provide guarantees. For example voting ballots on a desk can be changed by the people sitting behind them.

In contrast to inertness is transportation and the ability to move. Transportation of physical entities is limited by cost and time. To execute a physical attack, the attacker has to move herself physically to the spot, together with all the equipment that she needs: 
- In order to break a lock one has to travel to the lock itself.

- To commit voting fraud by impersonating a voter, one has to go to the polling station.

Transportation costs limit user's ability to transport entities such as books, enabling physical rights management. In addition to these, the attacker risks detection on the location of the attack, because she is visible.

- The material complexity of entities varies: some objects are easier to manufacture than others. Physical security would be radically different if persons could manufacture all physical entities easily. However, this requires physical equipment, which is visible and slow to transport. Thus we can be assured that the number of ballot boxes does not double suddenly, or that someone cannot create a network cable out of nothing. Rights management works because the manufacturing capabilities of individuals are limited. The most complex physical entities are persons: an underlying assumption behind most security systems is that persons cannot be duplicated. If this property would not hold, identification of persons through visibility would fail and no one could be held accountable.

- The last properties are contained in the visual cluster. Visibility of entities is determined by the properties entities themselves, such as size and shape, as well as by the observation capabilities of the persons looking at them. Concerning security, visibility gives assurance that entities are in a specific state and location. If this information confirms to a particular security goal, this gives assurance in the security of a system; if this is not the case, corrective action can be taken. Visibility is not always a positive contribution to security: several physical processes require invisibility or in information security terms - confidentiality. Examples of these are security implemented with locks and keys, paper containing votes and persons entering passwords on keyboards. Thus, it is limited visibility that enables most security processes: there is enough visibility to realize confidentiality, but not enough to reduce assurance.

Candidate clusters that were left out of the final results include "persons", "identities" and "transportation" and we chose to explain their properties in terms of other clusters. First, persons play a role in moving and modifying physical entities and conveying information to each other. Their location (or "presence") is important for live performances. Also persons are "non-deterministic". This characteristic makes it more difficult to script persons. Whereas a virus attack would work on any computer with a specific software program, a social engineering attack does not work in the same way every time it is attempted. However as the social domain is mostly excluded from the research as we focus on low 
level physical mechanisms this property is left out. Second, there is the concept of "identifiability". We see this as an emergent property of visibility, inertness and manufacturing capabilities: to identify something in the physical world requires it to be visible and in a steady shape, which is related to the inertness property. Finally manufacturing capabilities impact identification - easy copying casts doubt about the identification of entities. Third there is "transportation". We see this as included in the concept of independence, whether entities are capable of moving themselves or being moved by others.

This answers question RQ1 about the security relevant properties of physical entities.

\subsection{Properties of Digital Entities}

Figure 10.3 lists all the digital properties and their impact on security, together with the conditions under which these effects hold true.

\begin{tabular}{|c|c|c|c|}
\hline Property & Effect & Hypothesis & Conditions \\
\hline $\begin{array}{l}\text { Accurate Data } \\
\text { Storage, } \\
\text { Transportation and } \\
\text { Processing }\end{array}$ & $\mathrm{C}-$ & $\begin{array}{l}\text { Accuracy increases } \\
\text { possibility for transfer of } \\
\text { data outside of system or } \\
\text { abuse by an attacker. }\end{array}$ & $\begin{array}{l}\text { The system is compromised } \\
\text { or compromisable. } \\
\text { The system is connected. }\end{array}$ \\
\hline $\begin{array}{l}\text { Accurate Data } \\
\text { Storage, } \\
\text { Transportation and } \\
\text { Processing }\end{array}$ & $\mathrm{CIA}+$ & $\begin{array}{l}\text { Accuracy of IT increases } \\
\text { security. }\end{array}$ & $\begin{array}{l}\text { Data cannot be transported } \\
\text { out of the system. } \\
\text { The system is not } \\
\text { compromisable (controlled } \\
\text { by the organization setting } \\
\text { the policies that are accurate } \\
\text { and complete). }\end{array}$ \\
\hline $\begin{array}{l}\text { Accurate Data } \\
\text { Storage, } \\
\text { Transportation and } \\
\text { Processing }\end{array}$ & $\mathrm{I}-$ & $\begin{array}{l}\text { Accuracy makes record } \\
\text { forgery easy. }\end{array}$ & \\
\hline Fast Data Processing & A- & $\begin{array}{l}\text { Fast modification of data } \\
\text { makes abuse easy. }\end{array}$ & $\begin{array}{l}\text { The system is compromised } \\
\text { or compromisable. }\end{array}$ \\
\hline Fast Data Processing & $\mathrm{C}-$ & $\begin{array}{l}\text { Fast data processing makes } \\
\text { examining and stealing } \\
\text { confidential data easy. }\end{array}$ & $\begin{array}{l}\text { The system is compromised } \\
\text { or compromisable. }\end{array}$ \\
\hline Fast Data Processing & $\mathrm{I}-$ & $\begin{array}{l}\text { Fast data processing makes } \\
\text { changing a system's state } \\
\text { easy. }\end{array}$ & $\begin{array}{l}\text { The system is compromised } \\
\text { or compromisable. }\end{array}$ \\
\hline
\end{tabular}




\begin{tabular}{|c|c|c|c|}
\hline Property & Effect & Hypothesis & Conditions \\
\hline Fast Data Processing & $\mathrm{I}+$ & $\begin{array}{l}\text { System monitoring } \\
\text { increases integrity because } \\
\text { it detects errors and attacks. }\end{array}$ & $\begin{array}{l}\text { There is a remote site. } \\
\text { The system is distributed. } \\
\text { There is a trusted system } \\
\text { base that monitors the } \\
\text { system. }\end{array}$ \\
\hline Fast Data Storage & $\mathrm{A}+$ & $\begin{array}{l}\text { Fast data storage makes it } \\
\text { easy to keep data available. }\end{array}$ & \\
\hline Fast Data Storage & $\mathrm{C}-$ & $\begin{array}{l}\text { Fast data storage makes } \\
\text { sharing confidential } \\
\text { information easy. }\end{array}$ & $\begin{array}{l}\text { The system is compromised } \\
\text { or compromisable. }\end{array}$ \\
\hline Fast Data Storage & I+ & $\begin{array}{l}\text { Fast data storage allows a } \\
\text { defender to record logs of } \\
\text { activities and use them to } \\
\text { check for integrity errors } \\
\text { and attacks. }\end{array}$ & The system is isolated. \\
\hline $\begin{array}{l}\text { Fast Data } \\
\text { Transportation }\end{array}$ & $\mathrm{A}+$ & $\begin{array}{l}\text { Fast data transportation } \\
\text { makes it easy to store data } \\
\text { at a remote site. }\end{array}$ & The system is connected. \\
\hline $\begin{array}{l}\text { Fast Data } \\
\text { Transportation }\end{array}$ & $\mathrm{C}-$ & $\begin{array}{l}\text { Fast data transportation } \\
\text { makes it easy to store } \\
\text { confidential data at a } \\
\text { remote, uncontrolled site. }\end{array}$ & \\
\hline $\begin{array}{l}\text { Program } \\
\text { Computational } \\
\text { Complexity }\end{array}$ & $\mathrm{CI}+$ & $\begin{array}{l}\text { Cryptographic functions } \\
\text { keep data confidential. }\end{array}$ & \\
\hline $\begin{array}{l}\text { Program } \\
\text { Computational } \\
\text { Complexity }\end{array}$ & CIA- & $\begin{array}{l}\text { System complexity } \\
\text { increases the chance of } \\
\text { errors and abuse. }\end{array}$ & \\
\hline
\end{tabular}

Figure 10.3: Complete list of digital properties and their effects.

We observe three clusters of digital properties (Figure 10.4). Each cluster represents a related set of properties that are relevant for security. A general characterization - in line with earlier theories about the impact of automation - is that digital systems work fast and accurate but are complex. All of the underlying properties can be used by both attackers (having a negative effect on security) or by defenders (having a positive effect on security). If we consider the conditions under which these have a positive effect are that the initial system or a part of it can be trusted and is isolated or disconnected from other systems. Program complexity works asymmetric: for defenders, complexity allows for data encryption whereas complexity is also conducive to errors that help the attackers. This answers question $\mathbf{R Q 2}$ about the security relevant properties of digital entities. 


\begin{tabular}{ll}
\hline Cluster & Property \\
\hline Accuracy & $\begin{array}{l}\text { Accurate Data Storage, Transportation and } \\
\text { Processing }\end{array}$ \\
Complexity & Program Computational Complexity \\
Speed & Fast Data Processing \\
& Fast Data Storage \\
& Fast Data Transportation \\
\hline
\end{tabular}

Figure 10.4: Clusters of digital properties.

\subsection{Comparison between Physical and Digital Systems}

\subsubsection{Comparison on Goal Realization through Properties}

We now compare physical and digital security mechanisms on goal realization. As we already mentioned in Chapter 5 on Methods, results depend on goals, context and mechanisms. It is hard to come to universal conclusions as to whether physical or digital security is better in realizing security goals. They are different, and in this section we compare the physical and digital security mechanisms on goal realization. Figure 10.5 aggregates the results from all the case studies (Figures 6.15, 7.12, 8.20, and 9.15).

- First, for general security impact, we notice there are many more distinct security properties identified for physical security (both positive and negative) than for digital security. (This is also true for the specific security goals of confidentiality, integrity and availability discussed below.) The general defense mechanism in the digital domain is accuracy, which is offset by complexity: if systems accurately work as designed (including their programmed response attacks) they are secure. However system complexity leads to errors that can be exploited by attackers.

- Second, for confidentiality there is a positive effect of the environment through material properties, and (in)visibly, whereas digital systems mainly depend on complexity through cryptographic functions. Negative physical effects are present through weaknesses in the material properties and visibility, whereas digital effects mainly concern the capabilities of the attacker in terms of speed and accuracy of her tools.

- Third, for data integrity, integrity is positively impact by material properties and capabilities of the defender to monitor the system. In the physical world integrity is limited by decay and easy means to create objects; in the digital world it is affected by the capabilities of the attacker. 


\begin{tabular}{|c|c|c|c|}
\hline Security Goal & Effect & Physical Property & Digital Property \\
\hline \multirow[t]{2}{*}{ All } & + & $\begin{array}{l}\text { Active, Cohesive, Dense, } \\
\text { Identifiable, Inert, Limited } \\
\text { spacious, Movable, Slow, } \\
\text { Strong, Visible, Weak }\end{array}$ & $\begin{array}{l}\text { Accurate Data Storage, } \\
\text { Transportation and } \\
\text { Processing }\end{array}$ \\
\hline & - & $\begin{array}{l}\text { Active, Movable, Strong, } \\
\text { Visible }\end{array}$ & $\begin{array}{l}\text { Program Computational } \\
\text { Complexity }\end{array}$ \\
\hline \multirow[t]{2}{*}{ Confidentiality } & + & $\begin{array}{l}\text { Decaying, Difficultly } \\
\text { manufacturable, Enclosed } \\
\text { with opening, Invisible, } \\
\text { Modifiable, Uncopyable }\end{array}$ & $\begin{array}{l}\text { Program Computational } \\
\text { Complexity }\end{array}$ \\
\hline & - & $\begin{array}{l}\text { Easily manufacturable, } \\
\text { Modifiable, Sound } \\
\text { producing, Visible }\end{array}$ & $\begin{array}{l}\text { Accurate Data Storage, } \\
\text { Transportation and } \\
\text { Processing, Fast Data } \\
\text { Processing, Fast Data } \\
\text { Storage, Fast Data } \\
\text { Transportation }\end{array}$ \\
\hline \multirow[t]{2}{*}{ Integrity } & + & $\begin{array}{l}\text { Difficultly manufacturable, } \\
\text { Large, Modifiable }\end{array}$ & $\begin{array}{l}\text { Fast Data Processing, Fast } \\
\text { Data Storage, Program } \\
\text { Computational Complexity }\end{array}$ \\
\hline & - & $\begin{array}{l}\text { Decaying, Easily } \\
\text { manufacturable, Modifiable }\end{array}$ & $\begin{array}{l}\text { Accurate Data Storage, } \\
\text { Transportation and } \\
\text { Processing, Fast Data } \\
\text { Processing }\end{array}$ \\
\hline \multirow[t]{2}{*}{ Availability } & + & N/A & $\begin{array}{l}\text { Fast Data Storage, Fast Data } \\
\text { Transportation }\end{array}$ \\
\hline & - & Decaying & Fast Data Processing \\
\hline
\end{tabular}

Figure 10.5: Comparison of all physical and digital properties on their ability to realize security goals.

- Fourth, availability in the physical domain does not have a specific property that has a positive effect (this is aided by general impact of security properties in the first bullet). In the digital domain availability of data is aided by rapid distribution of data. Finally, availability in the physical domain is reduced by decay, and in the digital domain it is reduced by fast data processing by attackers who can rapidly delete data.

\subsubsection{Comparison for Case Studies}

We will now look deeper into the findings for our individual case studies. 
Access Control First, for access control, we concluded that physical access control is not fool-proof, attacks take time, and attackers risk detection. Physical security is layered and discretionary. The enforcement is distributed over many doors and locks, and the constructions are such that violations are always possible. By comparison, logical access control is very precise and less layered: either a person has access to an object or she does not. The reference monitor is a form of mandatory access control and must function perfectly, or else the system's security is completely breached. However logical security has three main advantages: first, management is centralized, and an administrator can uniformly configure access. Second, access by users can be logged and this increases accountability. Third, logical security policies are much more expressive. For example we cannot create a standard physical lock that can only be opened during office hours, but such fine-grained policies can be expressed in logical systems. These differences are explainable by the physical property clusters of inertness, transportation and architecture that slow down actions by both attackers and defenders, in contrast to the volatility and precision of digital systems.

Voting Second, for voting systems, we concluded that in the absence of hightech equipment such as cameras and sensors, the physical paper voting process can ensure adequate confidentiality and integrity. A direct comparison between completely electronic voting and paper voting leads to the conclusion that electronic voting systems score less on all security goals, because they cannot be inspected easily.

IT Infrastructure Third, for IT infrastructure, we concluded that physical mechanisms are especially beneficial when few changes are made to systems, whereas digital mechanisms are preferred when systems are very dynamic. The cost of changes (time, effort) for digital systems is much lower than for physical systems. However, because misconfigurations are always possible, the monitoring and auditing effort increase substantially for every physical security mechanism that is replaced by a digital mechanism.

Rights Management Fourth, for rights management, the effectiveness of mechanisms depends on the form of content. Digitally restricted music files are hard to secure, even using physical mechanisms, whereas access to music performances is easy to control physically.

This answers question RQ3. 


\begin{tabular}{|c|c|c|c|}
\hline \# & Explanation & Example & Place in Taxonomy \\
\hline S01 & $\begin{array}{l}\text { Logical system } \\
\text { sampling the } \\
\text { physical } \\
\text { environment. }\end{array}$ & LBAC & Location Dependent \\
\hline S02 & Parallel system & $\begin{array}{l}\text { Electronic voting next to digital } \\
\text { voting }\end{array}$ & Parallel \\
\hline S03 & Serial system & Vote printer & Serial \\
\hline S04 & $\begin{array}{l}\text { Processing } \\
\text { separation }\end{array}$ & $\begin{array}{l}\text { Virtual machines running on } \\
\text { different processors }\end{array}$ & Isolated \\
\hline S05 & Storage separation & $\begin{array}{l}\text { Virtual machines storing data on } \\
\text { different physical systems }\end{array}$ & Isolated \\
\hline S06 & Network separation & $\begin{array}{l}\text { Virtual machines running on } \\
\text { physically separated servers }\end{array}$ & Isolated \\
\hline S07 & $\begin{array}{l}\text { Implicit dependent } \\
\text { on data processing }\end{array}$ & CPU power & $\begin{array}{l}\text { Processing Power } \\
\text { Dependent }\end{array}$ \\
\hline S08 & $\begin{array}{l}\text { Implicit dependent } \\
\text { on data storage }\end{array}$ & Large data storage & $\begin{array}{l}\text { Data Storage } \\
\text { Dependeny }\end{array}$ \\
\hline S09 & $\begin{array}{l}\text { Implicit dependent } \\
\text { on data visualization }\end{array}$ & Specific visualizations & $\begin{array}{l}\text { Visualization } \\
\text { Dependeny }\end{array}$ \\
\hline S10 & $\begin{array}{l}\text { Implicit dependent } \\
\text { on data interactivity }\end{array}$ & Specific user interactions & $\begin{array}{l}\text { Interactivity } \\
\text { Dependency }\end{array}$ \\
\hline S11 & $\begin{array}{l}\text { Explicit dependent } \\
\text { on hardware }\end{array}$ & Specific hardware or network & Explicit Dependency \\
\hline $\mathrm{S} 12$ & Person dependent & Person & Person Dependent \\
\hline
\end{tabular}

Figure 10.6: Summary of hybrid system types. The last column lists the name in the taxonomy in Figure 10.7

\subsection{Combinations}

Figure 10.6 shows the list of hybrid systems that we encountered in the four case studies (Figures 6.18, 7.15, 8.23 and 9.20). We analyze these hybrid combinations to cluster these. At first, we observe very different patterns of integrating physical and digital security. In the case of access control, the integration takes the form of location-based access control, which ties access control decisions to the location of the user (and sometimes of the artifact she intends to manipulate). For voting, integration means automating only specific parts of the election process. For example, ballot counting is automated whereas vote casting is still done by hand. As for IT infrastructure, integration means using both physical and logical mechanisms for separating systems. For rights management, the possibility of combining physical and digital mechanisms depends on the type of contents. For digital content, integration means having it depend on physical limitations of 
hardware (such as processing speed) or on the availability of persons that use it (for online console games). Repeated analysis of these combinations and the security mechanisms discovered in the case studies led to the taxonomy in Figure 10.7 Hybrid systems are split into three types:

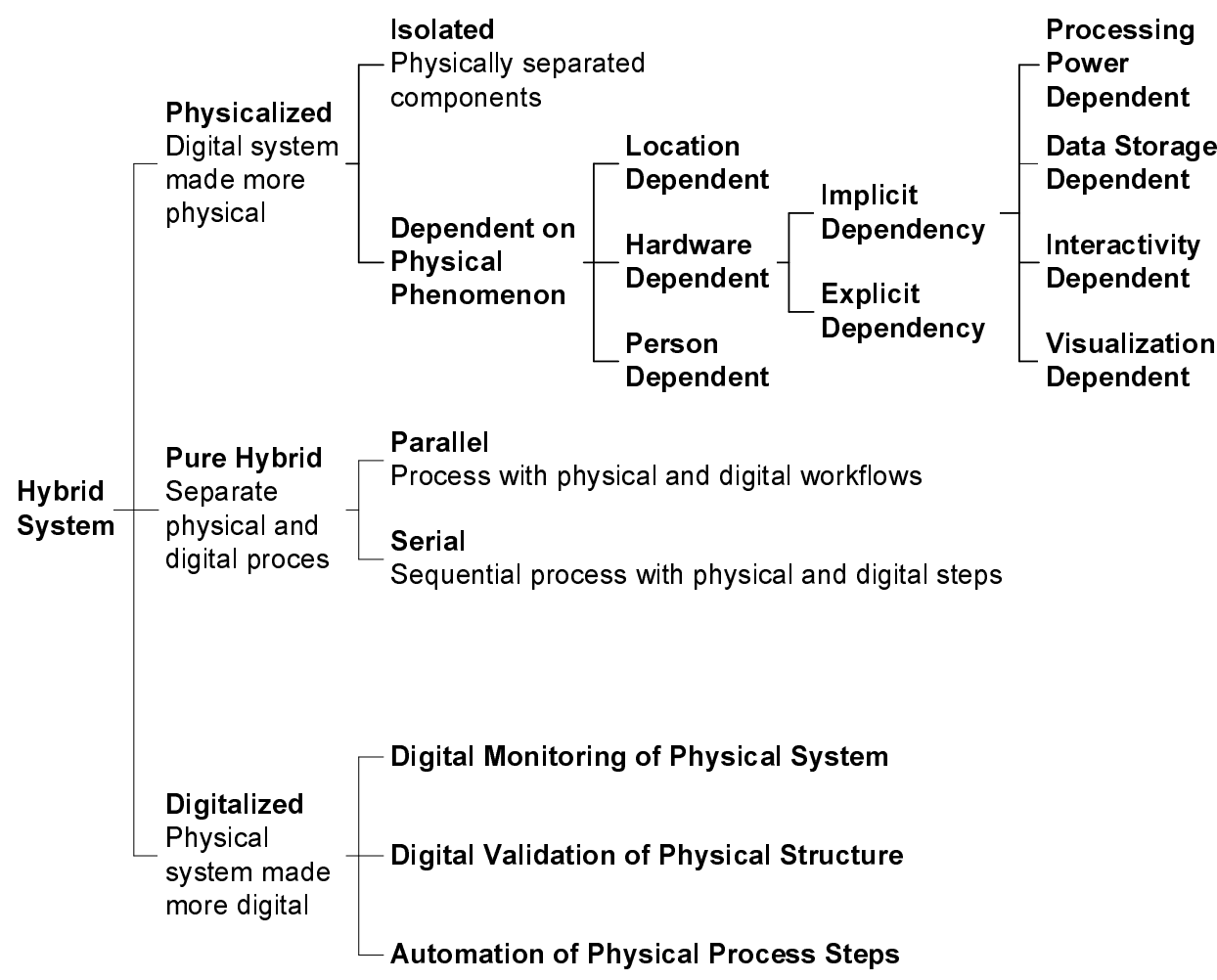

Figure 10.7: Taxonomy of hybrid systems.

1. physicalized systems that use physical protection mechanisms

2. pure hybrid systems, which combine physical and digital process steps

3. digitalized systems that use digital protection mechanisms

For physicalized systems there are two main hybrid forms: first there is isolation, found in the IT Infrastructure case study: processing, storage and communication. Second is dependence: grounding digital systems to physical phenomena. There are three main forms of dependencies:

1. location dependence (from case study on access control)

2. hardware dependence (from case study on rights management)

3. person dependence (from case study on rights management) 
Hardware dependence can further be split in explicit dependencies (hardware encryption such as TPMs and HSMs) or implicit dependencies, for example necessary storage, processing and communication. Dependencies on persons include all methods to keep a person in the loop, including strong authentication methods such as iris scanners and captchas.

There are also pure hybrid systems, which are derived from the voting case study:

1. parallel systems that execute physical and digital processes in parallel

2. serial systems that execute physical and digital steps consecutively

The principle behind parallelization is that each process is independent and distinct and have orthogonal failure modes or vulnerabilities to attacks. Serial systems are maybe best understood as the creation of composite materials: rather than using one type of material, multiple different layers are combined, resulting in a stronger overall process or structure.

Finally there are digitalized systems, and these form the counterpart of the first category: physical systems that are made more digital. To begin, there are existing monitoring solutions such as digital cameras in data centers and other types of sensors to detect activity. Cameras combine the physical property of visibility with the digital ability to monitor at a distance. The next two system types (validation and physical automation) are more speculative. The first system type arises out of our discussions with the partners from our virtualization case study. It could be possible to audit the IT structure inside a data center, by checking the physical connections between the components digitally. For example, if each cable and switch is equipped with RFID tags an engineer can scan the location of these tags and check if they are in the right place. This could greatly simplify auditing procedures and improve assurance. The second system type concerns physical automation and arises out of the case study on voting. Rather than making paper ballots digital and having a computer process them internally, we propose using techniques from industrial manufacturing to reduce errors in ballot transportation and counting. This "physical automation" could be useful for other situations as well and we will further explore this idea in Section 13.5 on Future Work.

Figure 10.8 extends the information in the taxonomy. For each node, an explanation is given, together with an explanation of the requirements as well as an example. This answers research question RQ4.

\begin{tabular}{|c|c|c|c|}
\hline Name & Explanation & Requirements & Example \\
\hline Physicalized & $\begin{array}{l}\text { Digital system made more } \\
\text { physical }\end{array}$ & $\begin{array}{l}\text { See Isolation and } \\
\text { Dependence }\end{array}$ & 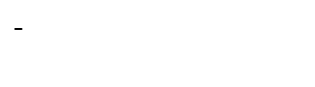 \\
\hline Isolation & $\begin{array}{l}\text { Physically separated } \\
\text { components }\end{array}$ & $\begin{array}{l}\text { Different pieces of } \\
\text { hardware }\end{array}$ & $\begin{array}{l}\text { Separate server racks in } \\
\text { data center }\end{array}$ \\
\hline
\end{tabular}




\begin{tabular}{|c|c|c|c|}
\hline Name & Explanation & Requirements & Example \\
\hline Dependence & $\begin{array}{l}\text { Dependence on physical } \\
\text { phenomenon }\end{array}$ & $\begin{array}{l}\text { See Location, } \\
\text { Hardware and Person }\end{array}$ & - \\
\hline Location & $\begin{array}{l}\text { Dependence on specific } \\
\text { location }\end{array}$ & $\begin{array}{l}\text { No specific } \\
\text { requirements }\end{array}$ & $\begin{array}{l}\text { Location-based access } \\
\text { control }\end{array}$ \\
\hline Hardware & $\begin{array}{l}\text { Dependence on specific } \\
\text { hardware }\end{array}$ & $\begin{array}{l}\text { See Implicit and } \\
\text { Explicit }\end{array}$ & DRM \\
\hline Person & $\begin{array}{l}\text { Dependence on specific } \\
\text { person }\end{array}$ & $\begin{array}{l}\text { Person is in the } \\
\text { transaction loop }\end{array}$ & Captcha \\
\hline Implicit & $\begin{array}{l}\text { Hardware implicitly } \\
\text { required }\end{array}$ & $\begin{array}{l}\text { Process is hardware } \\
\text { intensive }\end{array}$ & Apple iPad \\
\hline Explicit & $\begin{array}{l}\text { Hardware explicitly } \\
\text { required }\end{array}$ & $\begin{array}{l}\text { Hardware can be } \\
\text { secured }\end{array}$ & TPM, HSM \\
\hline $\begin{array}{l}\text { Processing } \\
\text { Power }\end{array}$ & Processing power required & $\begin{array}{l}\text { Process is computation } \\
\text { intensive }\end{array}$ & $3 \mathrm{D}$ rendering software \\
\hline Data storage & Data storage required & $\begin{array}{l}\text { Process is storage } \\
\text { intensive }\end{array}$ & $\begin{array}{l}\text { Google search engine } \\
\text { database }\end{array}$ \\
\hline Interactivity & Interactivity required & $\begin{array}{l}\text { Process requires } \\
\text { special user interaction }\end{array}$ & $\begin{array}{l}\text { Sony Playstation game } \\
\text { console }\end{array}$ \\
\hline Visualization & Visualization required & $\begin{array}{l}\text { Process outputs to } \\
\text { specific screen format }\end{array}$ & iPhone \\
\hline Pure hybrid & $\begin{array}{l}\text { Separate physical and } \\
\text { digital processes }\end{array}$ & $\begin{array}{l}\text { Transactions do not } \\
\text { have to occur } \\
\text { instantaneously }\end{array}$ & - \\
\hline Parallel & $\begin{array}{l}\text { Parallel process with } \\
\text { physical and digital work } \\
\text { flows }\end{array}$ & $\begin{array}{l}\text { Process has limited } \\
\text { number of transactions }\end{array}$ & $\begin{array}{l}\text { Two voting processes, } \\
\text { one electronic, one } \\
\text { physical }\end{array}$ \\
\hline Serial & $\begin{array}{l}\text { Sequential process with } \\
\text { physical and digital steps }\end{array}$ & $\begin{array}{l}\text { Process has limited } \\
\text { number of transactions }\end{array}$ & $\begin{array}{l}\text { Voting process with } \\
\text { printer and scanner }\end{array}$ \\
\hline Digitalized & $\begin{array}{l}\text { Physical system made } \\
\text { more digital }\end{array}$ & $\begin{array}{l}\text { See Monitoring, } \\
\text { Validation and Physical } \\
\text { automation }\end{array}$ & - \\
\hline Monitoring & Monitoring added & $\begin{array}{l}\text { Process should be } \\
\text { visible }\end{array}$ & $\begin{array}{l}\text { Voting process } \\
\text { monitored by camera }\end{array}$ \\
\hline Validation & $\begin{array}{l}\text { Validation of physical } \\
\text { structure }\end{array}$ & $\begin{array}{l}\text { Hardware should have } \\
\text { digital network }\end{array}$ & $\begin{array}{l}\text { Self auditing server } \\
\text { rack }\end{array}$ \\
\hline $\begin{array}{l}\text { Physical } \\
\text { automation }\end{array}$ & $\begin{array}{l}\text { Automation of physical } \\
\text { process steps }\end{array}$ & $\begin{array}{l}\text { Transactions do not } \\
\text { have to occur } \\
\text { instantaneously }\end{array}$ & $\begin{array}{l}\text { Automatic unloading } \\
\text { of ships with robots }\end{array}$ \\
\hline
\end{tabular}

Figure 10.8: Explanation of hybrid system taxonomy with examples of applications. 


\subsection{Trade-off Analysis}

\subsubsection{Trade-off Analysis on Properties}

Figure 10.9 and 10.10 show the trade-off analysis on the effect of physical and digital properties. The entries of the table are qualitative judgments based on our analysis of the case studies. Figure 10.11 aggregates the results of all case studies (Figures 6.22, 7.18, 8.26, and 9.23). Again, for the exact operationalization details used in the case studies, we refer to Figure 5.11 in Chapter 5 


\begin{tabular}{|c|c|c|c|c|c|c|c|c|c|c|c|c|c|}
\hline Hypothesis & $y^{2}$ & $8^{2}$ & $e^{e^{i c}}$ & $\mathrm{red}^{\mathrm{dx}}$ & $\mathrm{S}^{\mathrm{C}^{5}}$ & $e^{e^{5}}$ & $e^{e^{e}}$ & $s^{0}$ & $r^{e^{n}}$ & $\hat{y}^{x^{2}}$ & & & $e^{\theta^{8}}$ \\
\hline Active CIA+ & & $=$ & $=$ & $=$ & $=$ & $=$ & $=$ & $=$ & $=$ & + & + & + & + \\
\hline Active CIA- & & - & - & - & - & - & - & - & - & + & + & + & + \\
\hline Cohesive CIA+ & & & & & & & $=/+$ & $=/+$ & $=/+$ & $=$ & $=$ & $=$ & $=$ \\
\hline Decaying C+ & & & & & & & N/A & N/A & N/A & - & - & - & - \\
\hline Decaying IA- & $=/+$ & N/A & N/A & N/A & N/A & N/A & N/A & N/A & N/A & + & + & + & + \\
\hline Dense CIA+ & $=$ & $=$ & $=$ & $=$ & $=$ & $=$ & $=$ & $=$ & $=$ & $=$ & $=/-$ & $=$ & $=/-$ \\
\hline $\begin{array}{l}\text { Difficultly } \\
\text { manufacturable } \\
\text { CI+ }\end{array}$ & & $=/+$ & $=/+$ & $=/+$ & $=/+$ & $=/+$ & $=$ & $=$ & $=$ & $+/-$ & $+/-$ & $+/-$ & $+/-$ \\
\hline $\begin{array}{l}\text { Easily } \\
\text { manufacturable CI- }\end{array}$ & $=/+$ & $=$ & $=$ & $=$ & $=$ & $=$ & N/A & N/A & N/A & $+/-$ & $=$ & + & $=$ \\
\hline $\begin{array}{l}\text { Enclosed with } \\
\text { opening C+ }\end{array}$ & & $=$ & $=$ & $=$ & $=$ & $=$ & $=$ & $=$ & $=$ & $=$ & $=/-$ & $=$ & $=/-$ \\
\hline Identifiable CIA+ & & & & & & & - & - & - & $=$ & $=$ & $=/+$ & $=/+$ \\
\hline Inert CIA+ & & - & - & - & - & - & - & - & - & - & - & - & - \\
\hline Invisible C+ & $=/+$ & $=$ & $=$ & $=$ & $=$ & $=$ & $=$ & $=$ & $=$ & $=/+$ & $=/+$ & $=/+$ & $=/+$ \\
\hline Large I+ & & $=$ & $=$ & $=$ & $=$ & $=$ & $=$ & $=$ & $=$ & N/A & N/A & N/A & N/A \\
\hline $\begin{array}{l}\text { Limited spacious } \\
\text { CIA+ }\end{array}$ & $=$ & $=$ & $=$ & $=$ & $=$ & $=$ & $=$ & $=$ & $=$ & N/A & N/A & N/A & N/A \\
\hline Modifiable CI+ & & $=$ & $=$ & $=$ & $=$ & $=$ & $=$ & $=$ & $=$ & N/A & N/A & N/A & N/A \\
\hline Modifiable CI- & & $=$ & $=$ & $=$ & $=$ & $=$ & $=$ & $=$ & $=$ & N/A & N/A & N/A & N/A \\
\hline Movable CIA+ & & $=$ & $=$ & $=$ & $=$ & $=$ & $=/-$ & $=/-$ & $=/-$ & $=$ & $=$ & $=$ & $=$ \\
\hline Movable CIA- & & $=$ & $=$ & $=$ & $=$ & $=$ & $=/+$ & $=/+$ & $=/+$ & $=$ & $=$ & $=$ & $=$ \\
\hline $\begin{array}{l}\text { Slow CIA+ } \\
\text { Sound producing } \\
\text { C- }\end{array}$ & $=/+$ & $=/-$ & $=/-$ & $=/-$ & $=/-$ & $=/-$ & $=/-$ & $=/-$ & $=/-$ & $\begin{array}{l}=1- \\
=\end{array}$ & $\begin{array}{l}=/- \\
=\end{array}$ & $\begin{array}{l}=/- \\
=\end{array}$ & $\begin{array}{l}=/- \\
=\end{array}$ \\
\hline Strong CIA+ & $=$ & $=$ & $=$ & $=$ & $=$ & $=$ & $=/+$ & $=/+$ & $=/+$ & $=/+$ & $=/+$ & $=/+$ & $=/+$ \\
\hline Strong CIA- & $=/+$ & $=$ & $=$ & $=$ & $=$ & $=$ & $=/+$ & $=/+$ & $=/+$ & $=$ & $=$ & $=$ & $=$ \\
\hline $\begin{array}{l}\text { Uncopyable C+ } \\
\text { Visible C- }\end{array}$ & $=/+$ & $=$ & $=$ & $=$ & $=$ & $=$ & $\begin{array}{l}\text { N/A } \\
\text { N/A }\end{array}$ & $\begin{array}{l}\text { N/A } \\
\text { N/A }\end{array}$ & $\begin{array}{l}\text { N/A } \\
\text { N/A }\end{array}$ & $\begin{array}{l}- \\
=\end{array}$ & $\begin{array}{l}+/- \\
=\end{array}$ & $\begin{array}{l}+/- \\
=/+\end{array}$ & $\begin{array}{l}+/- \\
=\end{array}$ \\
\hline Visible CIA+ & $=$ & $=/-$ & $=/-$ & $=/-$ & $=/-$ & $=/-$ & - & - & - & $=$ & $=/-$ & - & $=/-$ \\
\hline Visible CIA- & + & $=$ & $=$ & $=$ & $=$ & $=$ & - & - & - & $=$ & $=$ & $=/+$ & $=$ \\
\hline Weak CIA+ & $=$ & $=$ & $=$ & $=$ & $=$ & $=$ & $=$ & $=$ & $=$ & $=$ & $=$ & $=$ & $=$ \\
\hline
\end{tabular}

Figure 10.9: Trade-off analysis on the impact of properties between hybrid and physical. Positive scores indicate that the hybrid system is more secure than the physical system, negative scores that it is not. N/A means that the effect was not observed in the case study. Empty cells indicate the property was not identified yet.(For the exact operationalization details, see Figure 5.11 in Chapter 5.) 


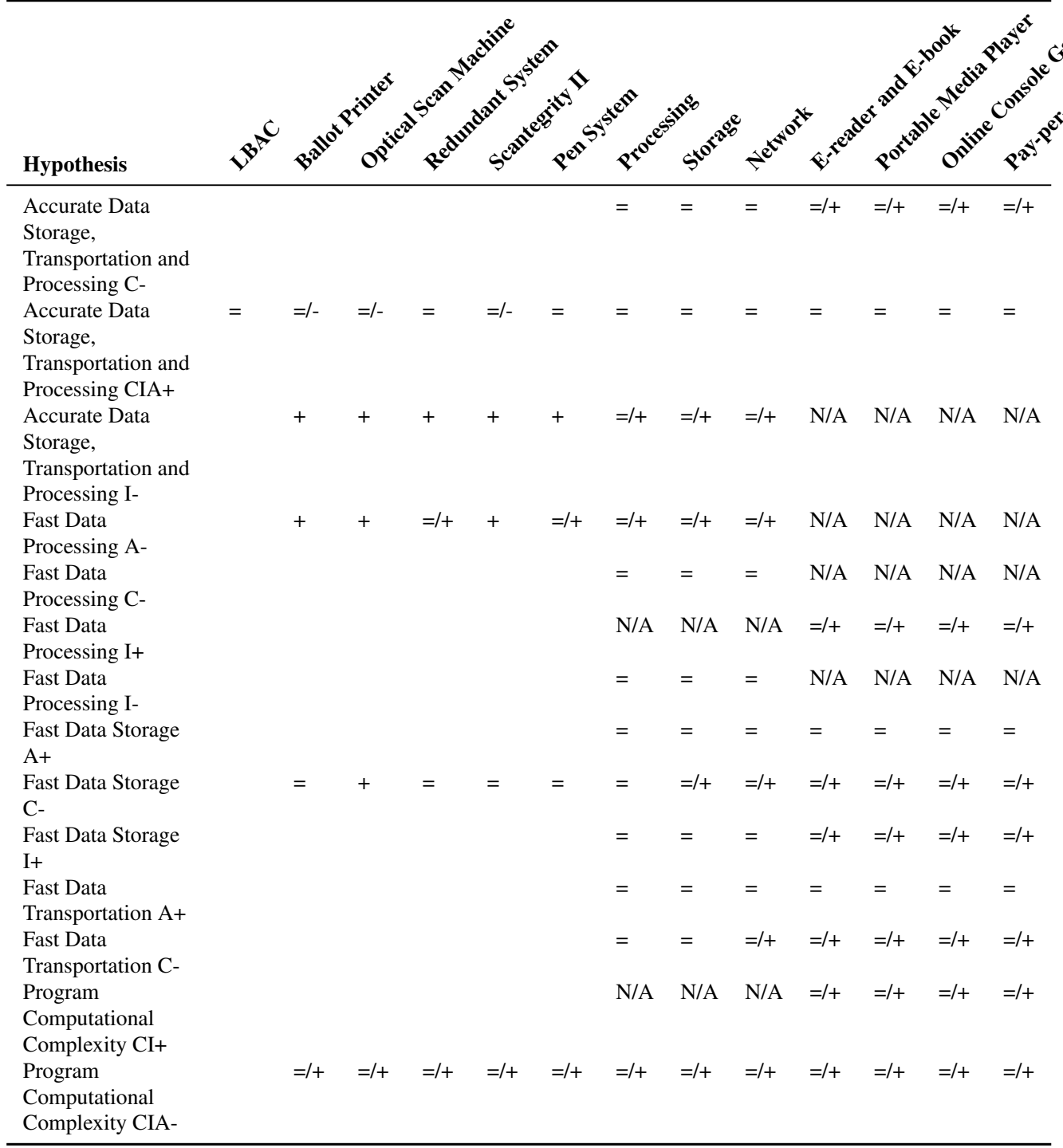

Figure 10.10: Trade-off analysis on the impact of properties between hybrid and digital. Positive scores indicate that the hybrid system is more secure than the digital system, negative scores that it is not. N/A means that the effect was not observed in the case study. Empty cells indicate the property was not identified yet.(For the exact operationalization details, see Figure 5.11 in Chapter 5) 


\subsubsection{Trade-off Analysis on Goals}

- Confidentiality Regarding confidentiality, we see that most hybrid systems score worse than physical systems, with the exceptions of access control and some rights management systems. The positive estimation for hybrid access control (LBAC) can be explained by the fact that the system as a whole works better whereas the security of data inside the system itself has not been considered. For the rights management systems, this can be attributed to a slightly different application of confidentiality: as mentioned for rights management systems confidentiality is better understood as limited availability. (See also Section 9.4.) Compared to digital systems, the evidence is clearer: hybrid systems score better on confidentiality.

- Integrity Regarding integrity, we see that hybrid systems score better than both physical and digital systems. The only \pm score comes from the comparison of hybrid IT infrastructure with physical infrastructure. This is caused by the fact that a hybrid system can easily be misconfigured such that it becomes as much volatile as a purely digital system.

- Availability Regarding availability, there is no clear winner if we decide between physical and hybrid systems. Hybrid systems for voting can score worse because they need both the physical and digital components to work. Otherwise LBAC can help to ensure security goals better than pure physical access control, and hybrid infrastructures replicate data better across different sites, while not becoming too volatile as some parts remain physical. The volatility of hybrid systems is also relevant if we compare them to digital systems: hybrid scores mostly better than pure digital systems because digital systems can be brought down more easily.

Figure 10.12 presents the conclusions in a qualitative way. 


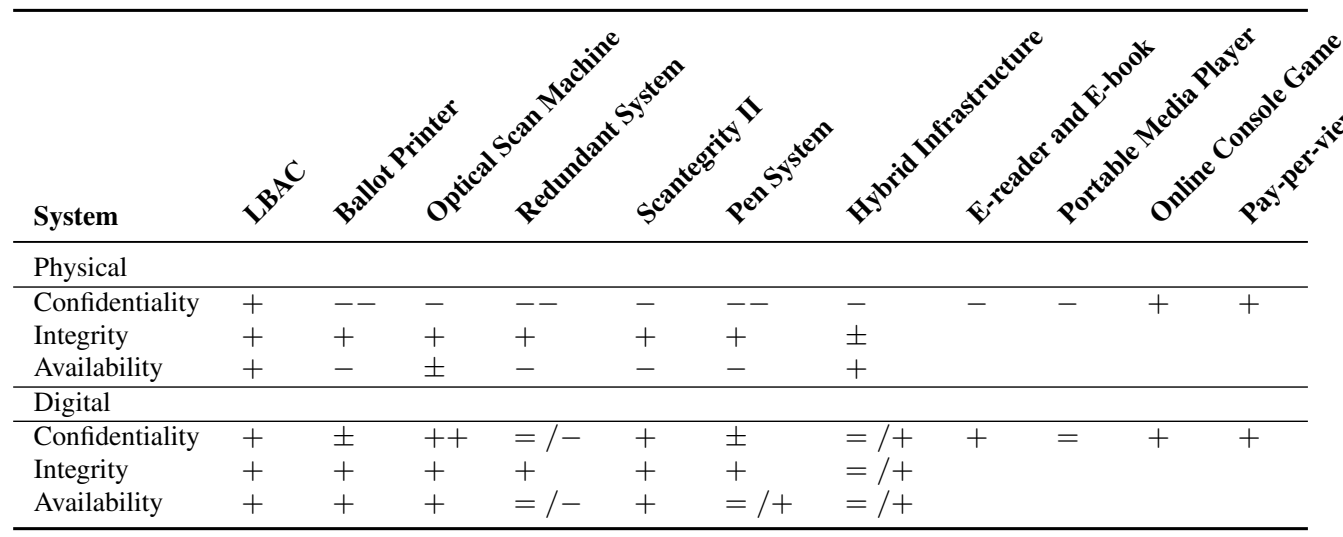

Figure 10.11: Hybrid systems compared to physical and digital systems.
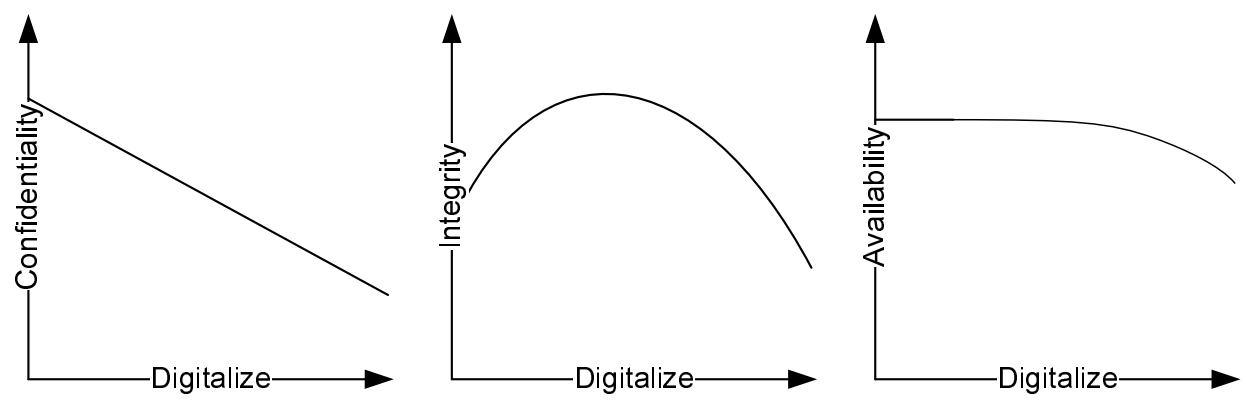

Figure 10.12: Qualitative representation of trade-offs for realizing security goals in physical, hybrid and digital systems, based on findings in Section 10.7 .

\subsubsection{Testing of Hypothesis for Hybrid Systems}

We will now also test the hypotheses posed in Chapter 5 about the benefits of hybrid systems using the results from Figure 10.11 .

H1 Accumulation of negative properties: a combination amplifies the worst properties of physical and digital systems.

For $\mathbf{H 1}$ to be accepted, the hybrid system should be worse than either a physical or digital system for a given goal. This hypothesis is clearly refuted: almost all hybrid systems score as good or better than their digital counterparts.

H2 Compensation: negative properties are limited by a positive property or vice versa.

For $\mathbf{H} \mathbf{2}$ to be accepted, the hybrid system's security should be between that of the related physical and digital system. (For a given goal it should score 
better than a digital system, but worse than a physical system or vice versa.) This hypothesis is supported by most of the cases shown in Figure 10.11 .

H3 Accumulation of positive properties: a combination amplifies the best properties of physical and digital systems.

For $\mathbf{H 3}$ to be accepted, the hybrid system should score better than both the physical and the digital system. Figure 10.11 shows some support for this hypothesis as well, especially for integrity.

\subsection{Validity}

Now that the results to the first five research questions have been presented, we can examine their validity. This is done by returning to the issues posed in Chapter 5: whether individual constructs are valid, and whether theoretical saturation has occurred, meaning if there is evidence that after adding information from each case, the amount of new information found in each subsequent case is diminished. This requires us first to define the constructs that form the results. In this thesis we have three types of results:

1. Properties of physical and digital entities

2. Taxonomy of hybrid systems

3. Comparison of physical and digital systems

Each of these constructs is different, and therefore we will also examine validity for each construct individually.

Properties of Physical and Digital Entities First, the hypotheses, namely the effects of the properties have all been demonstrated in the four case studies; they are analytically correct. If we consider the property of inertness, we can find all references to this property in the case studies. Second, the question can be posed whether there are other properties that have not been discovered in this research. This is again the issue of theoretical saturation. We examine this in Figure 10.13 which shows how many new hypothesis were identified in each case study.

If we consider the total number of new hypotheses for both the digital and physical domain, we observe a small increase in the number of new properties from the access control case to the voting case, after which the number of new properties decreases significantly. A likely explanation for initial increase is that there were no digital threats examined in access control. Had this been the case, there likely would have been no initial increase.

This gives some evidence that the hypotheses identified are capable to explain a large part of physical and digital security. However, the possibility exists that new properties and hypothesis can be found in future case studies. 


\begin{tabular}{llll}
\hline Case Study & $\begin{array}{l}\text { \# New Physical } \\
\text { Hypotheses }\end{array}$ & $\begin{array}{l}\text { \# New Digital } \\
\text { Hypotheses }\end{array}$ & Total \\
\hline Access Control & 12 & 1 & 13 \\
Voting & 10 & 4 & 14 \\
IT Infrastructure & 2 & 7 & 9 \\
Rights Management & 3 & 2 & 5 \\
\hline
\end{tabular}

Figure 10.13: Newly discovered hypothesis in each subsequent case study.

Taxonomy of Hybrid Systems Concerning the taxonomy, we can observe the different types of hybrid combinations. Items in the taxonomy can be traced back to the case studies. New case studies could potentially add more detail to the taxonomy (or possibly restructure it), but the taxonomy as it has been presented here is a valid for the case studies.

Comparison of Physical and Digital Systems Regarding the comparison, this constitutes a summary of the differences between physical and digital properties. Hence the same comments apply for the comparison.

Trade-off Analysis Trade-off analysis between digital and hybrid systems and between physical and hybrid systems is done transparently, and the rationale for each data point is explained. As such the conclusions are traceable from the evidence. Based on this evidence and argumentation, we conclude that there is sufficient evidence available to establish positive support of the three types of results.

\subsection{Conclusion}

In this chapter we integrated the results from the four case studies in a crosscase analysis. This allowed us to answered the first five the research questions:

RQ1: What are the properties of physical entities that physical security mechanisms depend upon?

Concerning the first research question, five clusters of physical security properties were discovered, namely the architectural, audible, independent, material and visual cluster. Properties are applicable (i) as requirements for physical security modeling, (ii) to construct security arguments and (iii) as targets for enhancement of security mechanisms. For example, now that it is known that inertness, visibility and other properties have a positive effect on security, engineers can develop systems that amplify those properties. 
RQ2: What are the properties of digital entities that digital security mechanisms depend upon?

As for the second research question, we identified five properties of digital systems: program computational complexity, Fast data processing, Fast data storage, Fast data transportation and finally Accurate data storage, transportation and processing. Similar to physical properties, we can design systems that enhance the positive effects of these properties.

RQ3: What are the key differences between physical and digital security mechanisms?

Regarding the third research question, we found that security goals are not uniformly better satisfied by either digital or physical systems. The exact security goals (confidentiality, integrity, availability) should be taken into account, as well as the particular context in which the system is functioning. Specific security-relevant characteristics of digital systems (as opposed to physical systems) include their volatility and their ability to monitor and keep track of history. These results are applicable for the prediction of the effect of automation, which is the removal of physical strength and weaknesses (the properties from the first research question) and the introduction of digital strengths and weaknesses. Second, engineers can use the knowledge to create stronger security mechanisms that embody strengths from both types.

RQ4: How can physical and digital security mechanisms be combined?

The answer of the fourth question is a taxonomy of possible types of hybrid systems. These are applicable as design patterns: generic solutions that engineers can apply in their system design. Vice versa, they are also useful to evaluate the security of existing systems. The relative security of hybrid systems compared to purely physical or digital systems is the answer to question four and it provides condensed advice to system architects:

- Purely digital systems should be avoided.

- Hybrid systems have better integrity than physical systems.

- The more important confidentiality is, the more physical mechanisms should be applied.

RQ5: What are the trade-offs between physical and digital mechanisms?

The answer to the fifth question about the trade-offs is shows in Figure 10.12. Confidentiality clearly requires systems to be more physical. Integrity is best aided by a hybrid system. Availability of a purely digital system is tends to be worse than that of a pure physical or hybrid system. 
In the remaining chapters, we will answer RQ6 (How can we apply the knowledge about physical and digital properties, combinations and trade-offs to improve security in an integrated way?) and reflect on the complete results. 


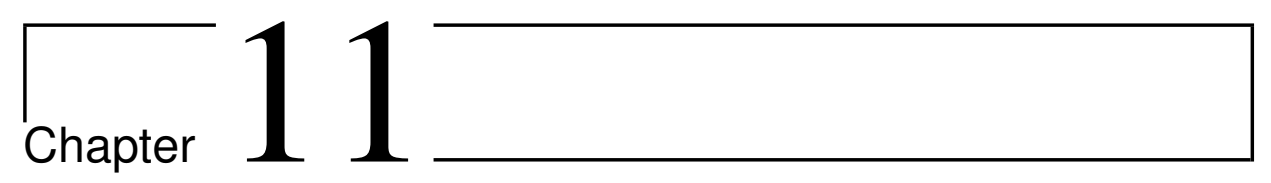

\section{Application of Results}

\subsection{Introduction}

With the main analytical results of the thesis being presented in Chapter 10 , we now turn to their practical application and answer the last research question (RQ6): How can we apply the knowledge about physical and digital properties, combinations and trade-offs to improve security in an integrated way? We do this in three steps. First, Section 11.2 explains how the answers to the research question can be applied. Second, Section 11.3 shows the implications of the research results to the four case studies: how we can improve on access control, voting systems, IT infrastructure and rights management. Fourth, Section 11.4 presents two new methods that can be used by practitioners, which can be used to apply the research results. The first, the "Integrated Risk Assessment Method" is intended for assessing the security of existing systems and uses the knowledge about security properties. The second, the "Pattern Library for Integrated Security Design" can be used for designing integrated secure systems. This method extends the results from RQ4 about types of hybrid systems by creating security patterns from them.

\subsection{Application per Research Question}

\subsubsection{Physical and Digital Properties}

First, the security properties listed earlier are requirements for physical security modeling. It has been demonstrated that they have an impact on security, and therefore every comprehensive physical security model should minimally incorporate these properties in order to produce meaningful results.

Second, the properties are useful to construct security arguments. ("Because a system uses certain properties, it is more secure.") Third, these properties are 
targets for enhancement of security mechanisms. Now that it is known that inertness, visibility and other properties have a positive effect on security, engineers can develop systems that amplify those properties or stimulate the conditions under which they are effective. For example to thwart off attackers who turn on cell phone cameras remotely, a small physical switch can be designed that can disconnect the power cable of a camera, rendering it inert, but also makes this disconnect visible through transparent plastic. If the user wants to ensure that her phone cannot take any pictures, she simply flips the switch. The need for such a mechanism is underscored by the fact that researchers are currently exploring malware that can even create a 3D model of the smartphone's environment [199].

\subsubsection{Comparison between Physical and Digital Systems}

First, knowledge of physical and digital security's strengths and weaknesses can be used to predict effects of automation: for example the Dutch public transport system, which was using paper tickets, has been automated and travelers now use contactless smart cards as payment method and ticker1. This automation has led to a number of surprises, which in retrospect were perfectly predictable using our results. We give two examples of these.

- When travelers pay in the old-fashioned way, the result is an inert and visible entity: the paper ticket. In the automated system, the payment becomes a dynamic and volatile process under the influence of the fast data processing property. First a traveler checks in using her smart card, and when she is finished with her journey she checks out. This flexible system introduces possible errors such as travelers checking in twice or forgetting to check out. A digital system can compensate for this using monitoring and auditing. However these mechanisms are cumbersome and ineffective for the user as she cannot easily check the card herself for errors (the visibility property is lost), and the public transport operators only refund a part of the money. With these differences in mind, the system architects could have realized the need for improved auditing and offered simple solutions to the users.

- The contactless smartcard turned out to be easily hacked, leading to public outrage. In retrospect this could have been foreseen by security experts simply applying logical thinking: IT systems have flaws and weaknesses (in this case weak, out-of-date, proprietary cryptography) which allows them to be hacked. Automation replaces ticket forgery (manufacturing property) with other types of fraud. However, the central transaction processing system that is available to the operators is capable of monitoring abuse and

\footnotetext{
${ }^{1}$ http://www.ovchipcard.nl
} 
limiting its impact, similar to banks monitoring transactions for indications of fraud. This feature was not known to the general public and this was one of the main reasons for outrage as they saw the systems as having failed completely. If instead it had been known earlier, the public's opinion might not have been so negative.

Second, engineers can use knowledge of physical and digital security strengths and weaknesses to create stronger security mechanisms. As an example we consider the problem of proving the absence or presence of network connections, outlined in Chapter $8{ }^{2}$ With existing technology, network connections can be either configured digitally (through a switch) or physically (through a patch cabinet). The former has the disadvantage of being changeable easily, as digital configurations are volatile, and the latter has the disadvantage of being hard to audit because cables need to be traced.

However with the knowledge of physical and digital security mechanisms, we can design a switch that is physically configurable, providing much more rigidity than current digital solutions, while still allowing for auditing and monitoring. This switch is shown in Figure 11.1. To connect different servers, the administrator has to physically push a button. In this example, servers connected to patch 1 and 4, and 2 and 3 are connected, indicated by the dark color of the (enabled) buttons on the right. Such a switch uses a new mixture of physical and digital separation, and combines the most beneficial properties of the physical and digital domain as shown in Figure 11.2 .

\subsubsection{Combinations}

The answer to question RQ4 is a taxonomy of possible types of hybrid systems, and an explanation of the requirements for applying each node. These are useful as design patterns: generic solutions that engineers can apply in their system design. Engineers can use Figure 10.8 to find the requirements for each pattern. (Section 11.4 presents the specific patterns for these.)

They are also useful to evaluate the security of existing systems. For example if experts intend to understand the security of the Dutch public transport system, they can scan for the presence of the patterns mentioned above.

\subsubsection{Trade-off Analysis}

The relative security of hybrid systems compared to purely physical or digital systems is the answer to question RQ5 and it provides condensed advice about trade-offs for system architects:

- Purely digital systems tend to be less secure than physical or hybrid ones.

\footnotetext{
${ }^{2}$ This example based on Van Cleeff et al. [208].
} 


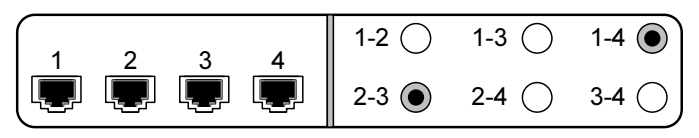

Figure 11.1: Schema of a physically configurable switch.

\begin{tabular}{lll}
\hline Domain & Property & Explanation \\
\hline Physical & $\begin{array}{l}\text { Strong } \\
\text { Inert }\end{array}$ & $\begin{array}{l}\text { Changes require a physical presence. } \\
\text { After clicking, the buttons remain in the } \\
\text { same position. } \\
\text { The active network connections can be } \\
\text { visually inspected. }\end{array}$ \\
Visible & $\begin{array}{l}\text { Accurate Data Storage, } \\
\text { The buttons are clearly indicated, it is not } \\
\text { necessary to swap cables in a patch } \\
\text { cabinet. } \\
\text { Trocessing } \\
\text { Accurate Data Storage, } \\
\text { Transportation and } \\
\text { Trocessing }\end{array}$ & \\
\hline
\end{tabular}

Figure 11.2: Beneficial physical and digital properties of the proposed switch.

- Hybrid systems have better integrity than physical systems.

- The more important confidentiality is, the more physical mechanisms should be applied.

\subsection{Application in Case Studies}

We now explain the direct relevance of the research results to each of the case studies.

\subsubsection{Access Control}

The research explains the security benefits of location-based access control, both from a theoretical perspective as well as through the identification of several use cases. It is one of the means to combine physical and digital security mechanisms that results in better security. Even though it is difficult to quantify the exact benefits of location-based access control, and maintenance is an issue, favoring it over pure logical access control can be recommended. 


\subsubsection{Voting}

Our research explains the difficulties that researchers have had in designing secure electronic voting systems. Paper has specific properties (inertness, visibility, foldable) that make it very suitable to solve the goal conflict between integrity and confidentiality, and electronic systems simply do not exhibit these properties. Governmental bodies face a choice between three options:

1. Hold completely paper-based elections. This choice is most logical if vote secrecy is seen as the core objective of voting next to integrity.

2. Automate small parts of the process such as counting or printing. The typical result is an increase of integrity and assurance, at the expense of some confidentiality.

3. The third option is to completely automate systems. This is possible if the confidentiality requirement is completely relaxed. When confidentiality is sacrificed, voting systems can be built that realize the goals of integrity and assurance to a much larger extent than otherwise possible.

\subsubsection{IT Infrastructure}

As for the design of virtualization technology itself, we recommend limiting the possibilities for introspection and intervention of VMMs, such that they cannot affect the application (threatening integrity) and cannot steal data from them (threatening confidentiality). If virtualization really is just an infrastructure layer, its functionality should simply focus on availability.

For applications running on a virtualized platform, we recommend to redesign them to facilitate batch processing and checkpointing. The problems of bookkeeping in a virtualized infrastructure are significant, and security can be improved by validating complete runs of a VM (from begin to end of a job), mitigating the problems that occur with restores and rollback operations.

For virtualization deployment and management, we recommend organizing it more strictly than an equivalent non-virtualized infrastructure. The virtualization infrastructure is much more powerful and should be considered to be a complete machine in itself, which should be put in a physically secured location, with tight security controls.

\subsubsection{Rights Management}

Our research clearly demonstrates that content protection depends on tying it to physical entities, and that in turn this ability depends on the type of content. Thus, creating effective general-purpose rights management systems seems infeasible. Enterprises wishing to protect their revenue streams should seek forms 
of content that are more difficult to copy, and we show patterns to achieve this. Live music, being authentic and time and location dependent, can be protected by physical means such as turnstiles and ticket control at the entrance of a music hall. However for the general content type of music, no effective rights management system can be built: no special devices are needed to play it, and the requirements in terms of data storage, transmission and processing (the patterns for grounding digital content) are minimal. Furthermore, music does not diminish quickly in value over time, and neither does it depend on any particular location where it can be used. We also explain the effectiveness of online console games in restricting copying: they tie the content to specific hardware, input devices and especially to persons, and use digital monitoring and auditing mechanisms. As such, online console games are excellent examples of combining physical and digital security mechanisms.

\subsection{Application in Risk Assessment and System Design}

This Section presents two new methods to apply the results of the thesis: one method for assessing risks and one set of security patterns for creating hybrid systems.

\subsubsection{Integrated Risk Assessment Method}

Figure 11.3 gives an overview of the steps in the first method. In essence,

(1) Model the system using KAOS including entities

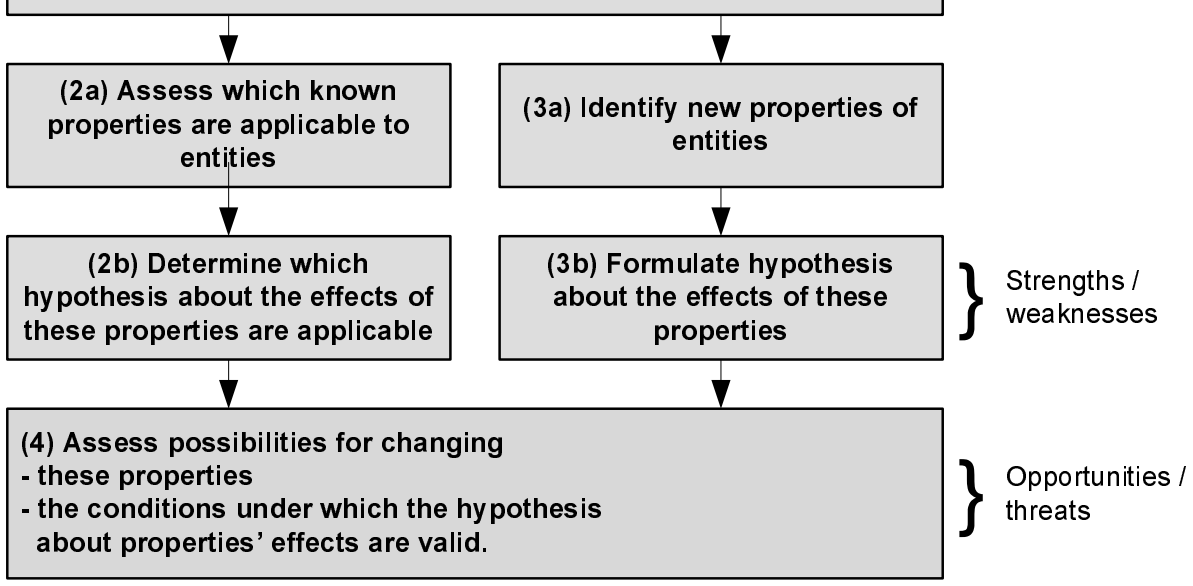

Figure 11.3: Structure of the integrated risk assessment method. 
the method constitutes a SWOT analysis $\sqrt[3]{3}$ To begin, we determine strengths and weaknesses in step two and three, by analyzing existing properties of entities and their impact on security. Last, we analyze possibilities to change the properties and their impact, resulting in an overview of opportunities and threats. We will now describe the method in more detail.

1. First, a model of the system is made using KAOS, as was done in the previous case studies following the method. This identifies the main security requirements that the system must fulfill and the entities that are involved in satisfying the requirements.

2. Second, we assess the effects that entities have on security using existing knowledge about the properties' effects.

(a) Assess which known properties are applicable to entities.

(b) Determine which hypothesis about the effects of these properties are applicable.

3. Third, we attempt to identify new properties and assess their effects on security. The detailed description for this is given in Sections 5.8.2 and 5.8.3. The method consists of two steps:

(a) Identify new properties of entities. We investigate how properties affect entities by considering how they (i) determine its possible states, (ii) help to realize operations on the entity, (iii) contribute to realizing security goals, (iv) help to resolve related conflicts between security goals, (v) play a part in the occurrence of threats, and (vi) contribute to the mitigation of threats.

(b) Next we create and update hypotheses about the effect of these properties, and the conditions under which these hypotheses hold, where each hypothesis has the following form:

$H x$ : physical security property has \{positive|negative $\}$ impact on security \{confidentiality, integrity, availability $\}$ under conditions $\{\mathrm{C}\}$

4. Fourth, for each entity, we assess possibilities for changing the properties and the conditions under which the hypothesis about properties' effects are valid. These changes can be positive (strengthen positive effects or weaken negative effects) or negative (weaken positive effects or strengthen negative effects).

${ }^{3}$ Cf. Den Braber et al. [46] for an earlier application of SWOT analysis in the domain of information security. 
5. Fifth, we create SWOT tables for each entity, using the results from step two, three and four.

We present a first validation of this method in Chapter 12 .

\subsubsection{Pattern Library for Integrated Security Design}

This section presents a library of security patterns that can be used to hybrid systems. Design patterns were introduced by Gamma et al. as a means to codify knowledge of successful software designs and facilitate their reuse [62]. Since their introduction in 1993, design patterns have been proposed for many different branches of computer science, including security. A summary of software security patterns is given by Dougherty et al. [51]. Patterns have even been used in the domains of physical security. For example Fernandez et al. presented patterns for Physical Access Control Systems [56]. Thus, to facilitate the usage of the knowledge developed in this thesis for system design, we transform the results of RQ4 (How can physical and digital security mechanisms be combined?) into a series of patterns. This is done by creating patterns from the leaves in the taxonomy that was the answer to this question, as illustrated in Figure 11.4. The patterns from the taxonomy are all presented in the format shown in Figure 11.5

In the next chapter, we present a first validation of this method. 


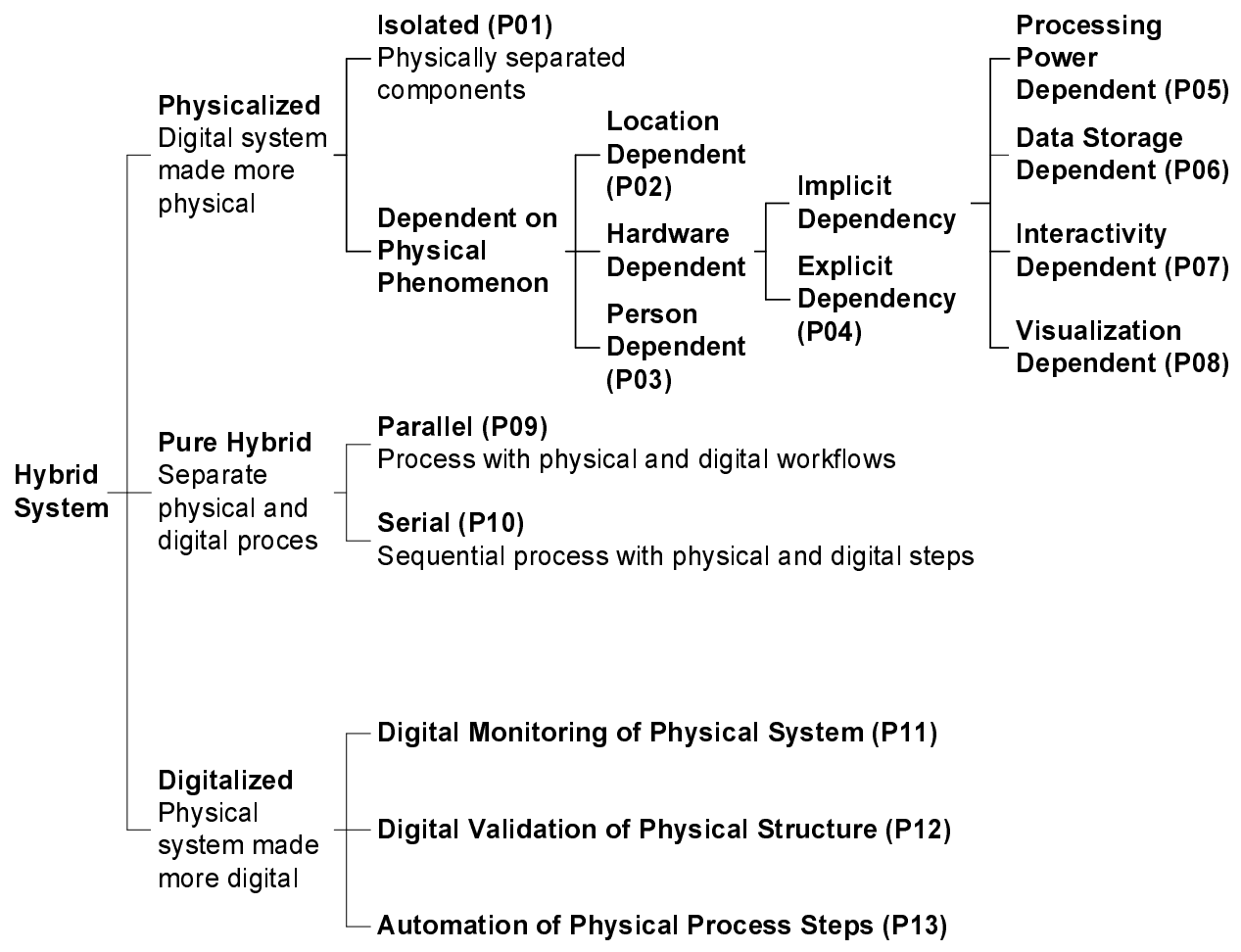

Figure 11.4: Taxonomy of patterns of hybrid systems. Each node lists its corresponding pattern ID. 


\begin{tabular}{|c|c|}
\hline Element & Description \\
\hline Explanation & Explanation of the pattern. \\
\hline Category & Place of the pattern in the taxonomy. \\
\hline Intent & Which problem is solved by the pattern. \\
\hline Example & $\begin{array}{l}\text { A real world example of the problem that can be solved by the } \\
\text { pattern. }\end{array}$ \\
\hline Applicability & $\begin{array}{l}\text { Description of requirements that a system must fulfill in order } \\
\text { to apply the pattern. }\end{array}$ \\
\hline Consequences & The positive and negative effects of the pattern. \\
\hline Implementation & Guidelines for the implementation of the pattern. \\
\hline Example Resolved & $\begin{array}{l}\text { Description of how the pattern solved the problem of the } \\
\text { example. }\end{array}$ \\
\hline Known Uses & Existing systems that apply the pattern. \\
\hline
\end{tabular}

Figure 11.5: Elements of a design pattern (adapted from Dougherty et al. [51].)

Next all patterns are further detailed in Figures 11.6.11.18.

\begin{tabular}{|c|c|}
\hline Pattern & Isolated system (P01) \\
\hline Explanation & Physically separated components \\
\hline Category & Physicalized digital system \\
\hline Intent & $\begin{array}{l}\text { Physically isolating systems reduces the chance that an attacker } \\
\text { can move from system to system. }\end{array}$ \\
\hline Example & $\begin{array}{l}\text { Attackers that have digital access to a system can use this } \\
\text { system as a stepping stone to attack other systems. }\end{array}$ \\
\hline Applicability & Different pieces of hardware \\
\hline Consequences & $\begin{array}{l}\text { Increased confidentiality and integrity, at the expense of } \\
\text { availability and maintainability }\end{array}$ \\
\hline Implementation & Separate systems physically from each other. \\
\hline Example Resolved & $\begin{array}{l}\text { A system is split into two or more physically isolated } \\
\text { components, such that an attacker can no longer move from } \\
\text { component to component using purely digital attack methods. }\end{array}$ \\
\hline Known Uses & Separate server racks in a data center \\
\hline
\end{tabular}

Figure 11.6: Isolated system pattern. 


\begin{tabular}{|c|c|}
\hline Pattern & Location dependent system (P02) \\
\hline Explanation & Process dependent on a particular location \\
\hline Category & $\begin{array}{l}\text { Physicalized digital system - dependent on physical } \\
\text { phenomenon }\end{array}$ \\
\hline Intent & $\begin{array}{l}\text { Making a system dependent on a particular location, meaning a } \\
\text { component or actor must be in a specific place for it to work, } \\
\text { increases the barrier for the attacker who cannot attack when } \\
\text { the component or actor is not in place. }\end{array}$ \\
\hline Example & $\begin{array}{l}\text { Systems are accessible from anywhere in the world, which } \\
\text { lowers the barrier for attackers to attack. }\end{array}$ \\
\hline Applicability & No specific requirements \\
\hline Consequences & $\begin{array}{l}\text { Reduces attacker surface at the expense of less ubiquitous } \\
\text { access by users }\end{array}$ \\
\hline Implementation & $\begin{array}{l}\text { Ensure that important transactions can only take place when a } \\
\text { component or actor is at a specific location. }\end{array}$ \\
\hline Example Resolved & $\begin{array}{l}\text { A system that allows critical transactions only to be executed in } \\
\text { specific locations, such that attackers cannot attack from } \\
\text { everywhere. }\end{array}$ \\
\hline Known Uses & Location-based access control \\
\hline
\end{tabular}

Figure 11.7: Location dependent system.

\begin{tabular}{|c|c|}
\hline Pattern & Person dependent system (P03) \\
\hline Explanation & Process dependent on a person or group of persons \\
\hline Category & $\begin{array}{l}\text { Physicalized digital system - dependent on physical } \\
\text { phenomenon }\end{array}$ \\
\hline Intent & $\begin{array}{l}\text { Making a system dependent on a person ensures that an attack } \\
\text { cannot be fully automated and thus raises the bar for an attacker. }\end{array}$ \\
\hline Example & $\begin{array}{l}\text { An attacker can script digital attacks in advance and execute } \\
\text { them against thousands of targets. }\end{array}$ \\
\hline Applicability & Person is in the transaction loop \\
\hline Consequences & Reduced attacker surface at the expense of slowdown \\
\hline Implementation & $\begin{array}{l}\text { Keep a person in the loop during transactions by asking input } \\
\text { that only a person can provide. }\end{array}$ \\
\hline Example Resolved & $\begin{array}{l}\text { A system that requires a person to interact with the system that } \\
\text { cannot be mimicked by a digital component, such that an attack } \\
\text { cannot be carried out fully automated. }\end{array}$ \\
\hline Known Uses & Captcha \\
\hline
\end{tabular}

Figure 11.8: Person dependent system. 


\begin{tabular}{|c|c|}
\hline Pattern & Explicit hardware dependent system (P04) \\
\hline Explanation & Process explicitly dependent on specific hardware \\
\hline Category & $\begin{array}{l}\text { Physicalized digital system - dependent on physical } \\
\text { phenomenon - hardware dependent }\end{array}$ \\
\hline Intent & $\begin{array}{l}\text { Making a system explicitly dependent on specific hardware } \\
\text { makes it difficult for an attacker to run a program on another } \\
\text { system, especially when it is not physically connected to the } \\
\text { original system. }\end{array}$ \\
\hline Example & $\begin{array}{l}\text { Cryptographic keys are necessary for secure operations but } \\
\text { must be stored closely to the system that needs them which } \\
\text { increases the chance of their compromise. }\end{array}$ \\
\hline Applicability & Hardware can be secured \\
\hline Consequences & $\begin{array}{l}\text { Increased confidentiality and integrity at the expense of } \\
\text { availability and maintainability }\end{array}$ \\
\hline Implementation & $\begin{array}{l}\text { Store specific components or data in a logically and physically } \\
\text { secured piece of hardware. }\end{array}$ \\
\hline $\begin{array}{l}\text { Example Resolved } \\
\text { Known Uses }\end{array}$ & $\begin{array}{l}\text { A system that securely stores cryptographic keys in hardware. } \\
\text { TPM, HSM }\end{array}$ \\
\hline
\end{tabular}

Figure 11.9: Explicit hardware dependent system.

\begin{tabular}{|c|c|}
\hline Pattern & Processing power dependent system (P05) \\
\hline Explanation & Process dependent on processing power \\
\hline Category & $\begin{array}{l}\text { Physicalized digital system - dependent on physical } \\
\text { phenomenon - hardware dependent - implicit hardware } \\
\text { dependent }\end{array}$ \\
\hline Intent & $\begin{array}{l}\text { Making a system dependent on the processing power makes it } \\
\text { difficult for an attacker to run a program on another platform, as } \\
\text { it must minimally have the same amount of computing power. }\end{array}$ \\
\hline Example & $\begin{array}{l}\text { Programs can be easily copied and run on thousands of systems, } \\
\text { even if a software license does not permit this. }\end{array}$ \\
\hline Applicability & Process is computation intensive \\
\hline Consequences & $\begin{array}{l}\text { Reduced ability to copy and run a system by attackers at the } \\
\text { expense of expensive hardware }\end{array}$ \\
\hline Implementation & $\begin{array}{l}\text { Design a system such that it can run only on specific high } \\
\text { performance hardware. }\end{array}$ \\
\hline Example Resolved & $\begin{array}{l}\text { A system that requires substantial computing resources only } \\
\text { available on specific hardware. }\end{array}$ \\
\hline Known Uses & 3D Rendering software \\
\hline
\end{tabular}

Figure 11.10: Processing power dependent system. 


\begin{tabular}{ll}
\hline Pattern & Data storage dependent system (P06) \\
\hline $\begin{array}{l}\text { Explanation } \\
\text { Category }\end{array}$ & $\begin{array}{l}\text { Process dependent on data storage } \\
\text { Physicalized digital system - dependent on physical } \\
\text { phenomenon - hardware dependent - implicit hardware } \\
\text { dependent } \\
\text { Making a system dependent on large data storage makes it } \\
\text { difficult for an attacker to move it to another system because it } \\
\text { must minimally have the same data storage capacity. }\end{array}$ \\
Intent & $\begin{array}{l}\text { A program that requires little data storage space is very easy to } \\
\text { copy. }\end{array}$ \\
Example & $\begin{array}{l}\text { Process is storage intensive } \\
\text { Reduced ability to copy and run a system by attackers at the } \\
\text { expense of an expensive storage system }\end{array}$ \\
Consequences & $\begin{array}{l}\text { Design a system such that it can only run using a large amount } \\
\text { of storage with specific data. }\end{array}$ \\
Amplementation & $\begin{array}{l}\text { A program that is dependent on a large amount of data in order } \\
\text { to execute. } \\
\text { Google search engine database }\end{array}$ \\
Example Resolved
\end{tabular}

Figure 11.11: Data storage dependent system.

\begin{tabular}{|c|c|}
\hline Pattern & Interactivity dependent system (P07) \\
\hline Explanation & Process dependent on interactive usage \\
\hline Category & $\begin{array}{l}\text { Physicalized digital system - dependent on physical } \\
\text { phenomenon - hardware dependent - implicit hardware } \\
\text { dependent }\end{array}$ \\
\hline Intent & $\begin{array}{l}\text { Making a system dependent on interaction with a person and } \\
\text { the environment ensures that the attacker cannot recreate the } \\
\text { same system using purely digital components. }\end{array}$ \\
\hline Example & $\begin{array}{l}\text { A program that interacts only using standard means (keyboard, } \\
\text { mouse) can be easily ported and copied to other systems. }\end{array}$ \\
\hline Applicability & Process requires special user interaction \\
\hline Consequences & $\begin{array}{l}\text { Reduced ability to copy and run a system by attackers at the } \\
\text { expense of a possibly complicated user interface. }\end{array}$ \\
\hline Implementation & $\begin{array}{l}\text { Design a system such that it offers users specific devices to } \\
\text { provide input that are difficult to duplicate. }\end{array}$ \\
\hline Example Resolved & $\begin{array}{l}\text { A program that is dependent on specific user interactions using } \\
\text { specific devices. }\end{array}$ \\
\hline Known Uses & Sony PlayStation game console \\
\hline
\end{tabular}

Figure 11.12: Interactivity dependent system. 


\begin{tabular}{|c|c|}
\hline Pattern & Visualization dependent system (P08) \\
\hline Explanation & Process dependent on specific visualization \\
\hline Category & $\begin{array}{l}\text { Physicalized digital system - dependent on physical } \\
\text { phenomenon - hardware dependent - implicit hardware } \\
\text { dependent }\end{array}$ \\
\hline Intent & $\begin{array}{l}\text { Making a system dependent on specific visualization } \\
\text { capabilities makes it difficult for an attacker to run a program } \\
\text { on another platform, as it must minimally have the same } \\
\text { visualization capabilities. }\end{array}$ \\
\hline Example & $\begin{array}{l}\text { A program that has no specific visualization requirements can } \\
\text { be easily ported and copied to other systems. }\end{array}$ \\
\hline Applicability & Process outputs to specific screen format \\
\hline Consequences & $\begin{array}{l}\text { Reduced ability to copy and run a system by attackers and the } \\
\text { expense of portability }\end{array}$ \\
\hline Implementation & $\begin{array}{l}\text { Design a system such that it depends on specific screens such } \\
\text { that displaying it on other systems causes a reduced user } \\
\text { experience. }\end{array}$ \\
\hline Example Resolved & A program that only runs in specific screen modes. \\
\hline Known Uses & iPhone \\
\hline
\end{tabular}

Figure 11.13: Visualization dependent system.

\begin{tabular}{ll}
\hline Pattern & Parallel system (P09) \\
\hline $\begin{array}{l}\text { Explanation } \\
\text { Category }\end{array}$ & $\begin{array}{l}\text { Process with physical and digital workflows } \\
\text { Intent }\end{array}$ \\
$\begin{array}{l}\text { Pure hybrid system } \\
\text { Implementing a process both physically and digitally ensures } \\
\text { that an attacker must break both the physical and digital } \\
\text { security mechanisms to attack the system successfully. } \\
\text { A pure digital or physical voting system can be attacked by } \\
\text { either digital or physical means. }\end{array}$ \\
$\begin{array}{l}\text { Applicability } \\
\text { Consequences }\end{array}$ & $\begin{array}{l}\text { Reduced attack surface at the expense of supporting two } \\
\text { processing streams and reconciliation effort }\end{array}$ \\
Implementation & $\begin{array}{l}\text { Split a system in a separate physical and digital process that run } \\
\text { in parallel but perform the same transactions and reconcile } \\
\text { differences at the end. }\end{array}$ \\
Example Resolved & $\begin{array}{l}\text { A system that executes a physical and digital process in parallel. } \\
\text { Two voting processes, one digital, one physical }\end{array}$ \\
Known Uses &
\end{tabular}

Figure 11.14: Parallel system. 


\begin{tabular}{|c|c|}
\hline Pattern & Serial system (P10) \\
\hline Explanation & Sequential process with physical and digital steps \\
\hline Category & Pure hybrid system \\
\hline Intent & $\begin{array}{l}\text { Implementing a process sequentially using physical and digital } \\
\text { steps makes it more difficult for an attacker to attack the system } \\
\text { using either physical or digital means. }\end{array}$ \\
\hline Example & $\begin{array}{l}\text { A pure digital or physical voting system can be attacked by } \\
\text { either digital or physical means. }\end{array}$ \\
\hline Applicability & Process has limited number of transactions \\
\hline Consequences & $\begin{array}{l}\text { Increased ability to detect attacks at the expense of slowdown } \\
\text { due to the physical process steps }\end{array}$ \\
\hline Implementation & $\begin{array}{l}\text { Split a system in physical and digital steps using devices such } \\
\text { as printers and scanners. }\end{array}$ \\
\hline Example Resolved & A system that executes physical and digital steps subsequently. \\
\hline Known Uses & Voting process with printer and scanner \\
\hline
\end{tabular}

Figure 11.15: Serial system.

\begin{tabular}{|c|c|}
\hline Pattern & Digital monitoring of physical system (P11) \\
\hline Explanation & Digitally monitored physical process \\
\hline Category & Digitalized physical system \\
\hline Intent & $\begin{array}{l}\text { Digitally monitoring a physical system ensures that an attacker } \\
\text { cannot change a physical system without this being detected } \\
\text { digitally. }\end{array}$ \\
\hline Example & $\begin{array}{l}\text { A physical voting system must be monitored by persons and is } \\
\text { subject to their limited capabilities, increasing the chance of } \\
\text { errors and attacks. }\end{array}$ \\
\hline Applicability & Process should be visible \\
\hline Consequences & $\begin{array}{l}\text { Increase assurance of correct process execution at the expense } \\
\text { of a reduction in privacy of users }\end{array}$ \\
\hline Implementation & $\begin{array}{l}\text { Monitor a physical system using digital cameras which feed } \\
\text { information to a system that can detect errors or attacks. }\end{array}$ \\
\hline Example Resolved & $\begin{array}{l}\text { A system that monitors a physical system and detects errors and } \\
\text { attacks. }\end{array}$ \\
\hline Known Uses & Voting process monitored by a camera \\
\hline
\end{tabular}

Figure 11.16: Digital monitoring of physical system. 


\begin{tabular}{|c|c|}
\hline Pattern & Digital validation of physical structure (P12) \\
\hline Explanation & Digitally validated physical system \\
\hline Category & Digitalized physical system \\
\hline Intent & $\begin{array}{l}\text { Digitally validation of a physical system ensures that an } \\
\text { attacker cannot change a physical system without this being } \\
\text { detected digitally. }\end{array}$ \\
\hline Example & $\begin{array}{l}\text { A physical system such as a server rack with servers is difficult } \\
\text { to audit because the physical connections are hard to trace. }\end{array}$ \\
\hline Applicability & Hardware should have digital network connections \\
\hline Consequences & $\begin{array}{l}\text { Increased assurance of the correct construction of a physical } \\
\text { system at the expense of using more hardware }\end{array}$ \\
\hline Implementation & $\begin{array}{l}\text { Add sensors to a physical infrastructure and let the sensors } \\
\text { connect to each other such that they form a network and audit } \\
\text { the network structure. }\end{array}$ \\
\hline Example Resolved & A system that validates a physical structure. \\
\hline Known Uses & Self-auditing server rack \\
\hline
\end{tabular}

Figure 11.17: Digital validation of physical structure.

\begin{tabular}{|c|c|}
\hline Pattern & Automation of physical process steps (P13) \\
\hline Explanation & Automated physical process \\
\hline Category & Digitalized physical system \\
\hline Intent & $\begin{array}{l}\text { Automating physical process steps ensures that the process is } \\
\text { carried out precisely but can be audited visually, thus making it } \\
\text { more difficult for the attacker to change the system. }\end{array}$ \\
\hline Example & $\begin{array}{l}\text { Executing a physical process is error prone and subject to } \\
\text { physical attacks by the persons involved. }\end{array}$ \\
\hline Applicability & Transactions do not have to occur instantaneously \\
\hline Consequences & $\begin{array}{l}\text { Increased assurance of the correct execution of a physical } \\
\text { process at the expense of using more hardware }\end{array}$ \\
\hline Implementation & $\begin{array}{l}\text { Use physical machines such as robots to automate a physical } \\
\text { process. }\end{array}$ \\
\hline Example Resolved & $\begin{array}{l}\text { A system that automates a physical process by physically } \\
\text { manipulating objects. }\end{array}$ \\
\hline Known Uses & Automatic unloading of ships with cranes \\
\hline
\end{tabular}

Figure 11.18: Automation of physical process steps. 


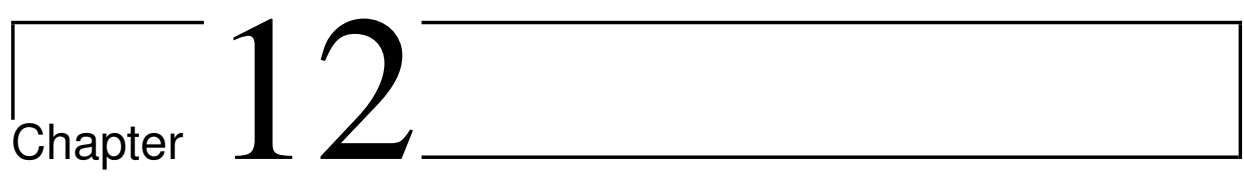

\section{Validation in Focus Group Meeting}

\subsection{Introduction}

In this chapter we summarize the results of the focus group meeting, in which the two methods presented in the previous chapter are evaluated. This is done with three objectives in mind: the first objective is to understand the current state of the practice regarding physical and digital security: if more is known about the state of practice it becomes easier to assess the practical benefits of the research results because a baseline is established. The second objective of the focus group meeting was to evaluate the usefulness of the main research results, being the method to analyze the security of systems ("method one") and the method to design integrated systems ("method two"). The third objective concerned the opportunities for improving the research results based on the recommendations of the participants. Thus we have three objectives for the focus group meeting, construed as empirical questions about opinions and responses of the participants:

FGRQ1 What is the state of the practice of the participants and how do they currently deal with digital and physical security?

FGRQ2 How useful are the developed methods?

FGRQ3 How can the methods be improved?

Before answering these questions we will first explain the research methods and the underlying conceptual framework. 


\subsection{Method}

\subsubsection{Conceptual Framework}

\section{Usefulness}

Following the framework that Nielsen developed for user experiences [147], we define usefulness as a combination of utility and usability of a design for the intended user group. Utility concerns whether the design provides the features that are necessary, and usability is the measure how easy and pleasant the design is to use. Usability has five quality components:

1. Learnability: How quickly users can apply the design after being exposed to them.

2. Efficiency: How quickly users can perform tasks.

3. Memorability: How easy it is for users to remember the design and reuse it.

4. Errors: The number of errors, their severity and the possibility to recover from them.

5. Satisfaction: The extent to which the design is pleasant to use.

Naturally this framework must be adapted and operationalized for our research purpose. First, this concerns the object of study itself: the primary outcome is not a web user interface design with specific features, but two methods aimed at improving information security: the artifact to be investigated is not a design but the research results themselves. Second, we must consider the opportunities and limitations of a focus group design:

- Regarding utility, we operationalize it as whether the methods can help to assess and improve information security.

- As for usability, because we limit the design to one session, we consider memorability not measurable and will exclude it from the evaluation. As for the other quality components, we operationalize them in the following way:

- Learnability: the time spent on the teaching the methods to users after which they can apply them independently.

- Efficiency: the time spent on performing the tasks.

- Errors: the number of errors users made in applying the methods.

- Satisfaction: general feedback on the research results. 


\section{Focus Group Meetings}

The main research method used is that of the focus group meeting [147]. In a focus group meeting, several users are brought together and studied or questioned about a particular artifact. Typically a focus group meeting takes place in the form of discussions, however in this case we asked the participants to perform specific exercises and studied if and how they were able to complete them.

\subsubsection{Meeting Design and Preparation}

\section{Meeting Structure}

As we opted for a physical meeting of the participants, there was only limited time available for evaluation, and a balance had to be struck between sufficient depth (attention to details) and breadth (coverage of the results). We chose therefore for two types of data collection:

- Survey for collecting general information (breadth)

- Exercises to collect data about the two methods (depth)

To save further time, the exercises concerned a case with which most of the participants were likely to be familiar, namely the Dutch OV Chipcard, which is a contactless smartcard solution in use by public transport in the Netherlands (bus, tram, metro, train). The solution replaced older paper tickets and its security has been widely discussed, especially after Dutch researchers managed to hack the Mifare classic chip on the tickets [98].

The structure of the meeting was as follows:

1. Introduction: we gave a general introduction into the topic and the objectives of the meeting.

2. Pretest survey: we acquired data about current practices and opinions about physical and digital security through a survey.

3. Execution: we explained the two methods and gave exercises to the participants, after which we discussed the results.

4. Post-test survey: we explicitly tested the usefulness and usability of the results.

5. Closure: we asked feedback and discussed three topics related to physical and digital security that recently had been mentioned in the news:

- A heist of Bitcoins

- The possible reintroduction of electronic voting in the Netherlands 
- The advise by the Dutch police to tape off web cams to prevent being recorded by hackers

The survey itself is listed in Appendix A

\section{Meeting Trial}

Prior to the actual focus group meeting we held a trial at the University of Twente on January 8th 2014. The objective of this session was to test the focus group setup:

- Detect and correct errors in the material (presentation and questionnaire).

- Clarify issues whenever there was the chance of confusion.

- Test the feasibility of completing the exercises in the available time.

This meeting attracted three participants and finished in time. Based on the feedback the overall meeting outline was deemed appropriate. Changes were made however to the material, specifically to reduce the amount of necessary reading, and to prevent possible confusion of the participants.

\section{Finding Participants}

The objective was to gather ten to twenty information security professionals from the industry to comment on the thesis results. To find participants, we followed several strategies:

- LinkedIn Groups. We posted a message on LinkedIn security groups (Cyber Security Research Platform ${ }^{1}$

- LSEC. LSEC ("Leaders in Security") $2^{2}$ is a non-profit organization consisting of security professionals. On request it forwarded our invitation to its members.

- Security mailing lists. We put out an email to the Dutch security research mailing list Safe-n ${ }^{3}$.

- Existing network. We contacted existing persons of our network within the industry from earlier security research projects from the University of Twente.

\footnotetext{
${ }^{1}$ http://www.linkedin.com/groups/Cyber-Security-Research-Platform-4362045/about

${ }^{2}$ https://leadersinsecurity.org

${ }^{3}$ Safe-nlsafe-nl@science.ru.nl
} 


\subsubsection{Data Analysis}

The raw data results of the exercises and survey were put into a spreadsheet. Next these results were processed. Specifically actions done were:

- Correcting for spelling errors;

- Translating Dutch sentences into English;

- Harmonizing results of different participants to make them comparable.

- Interpreting the results. A specific action was the interpretation of nonresponse and its effect on the results.

- Answering the research questions. (Figure A.1 in the appendix shows how the questions relate to the research questions.)

\subsection{Results}

This section presents a summary of the results. (The complete results are available in Appendix A.)

\subsubsection{Meeting Attendance and Duration}

The different channels that we used to attract participants had different success rates. The most participants came from the existing network, whereas the LinkedIn group was the least successful because the postings triggered a spam filter after which the messages awaited moderation by the group owners. In total eleven persons participated in the meeting that was held on March 27th 2014. Figure 12.1 shows the background of the participants.

The list shows that most of the participants were very experienced (some more than 20 years) in information security and had a diverse background, with the largest subgroup originating from the IT industry itself. The participants received a presentation about the subject of about 30 minutes. This was followed by 90 minutes of exercises, and each exercise included a short explanation about 5 minutes. The time spent teaching (relevant for the learnability subgoal) was thus limited as was the time spent making the exercises (efficiency goal).

\subsubsection{State of the Practice}

We present the answers that are relevant for the focus group first research question FGRQ1 about the state of the practice.

To begin, we asked the participants how the perform physical and/or digital security assessments. Most participants choose to use ISO 27001/27002 for this 


\begin{tabular}{llll}
\hline$\#$ & Sector & Position & Experience (years) \\
\hline 1 & Banking & Information Security Officer / Adviser & 13 \\
2 & Diversified & Security Researcher & 25 \\
3 & IT Industry & Security Consultant & 15 \\
4 & IT Industry & Corporate Security & $>20$ \\
5 & Chemical Industry & Corporate Security Officer & 9 \\
6 & Insurance & Security Officer & 10 \\
7 & IT Industry & Software Architect / Developer & 0 \\
8 & Diversified & R\&D Manager & 15 \\
9 & Banking & Security Adviser & 4 \\
10 & Utility & Security Consultant & 3 \\
11 & Academia & R\&D Coordinator & 18 \\
\hline
\end{tabular}

Figure 12.1: Details of focus group meeting participants.

(6 for physical and 7 for digital security), with others being ISF (2x), ISA 99 and Department of Homeland Security (DHS) or simply outsourcing the assessment.

Another question concerned the experience that participants had with physical and digital security. The results indicated that the participants have the most experience in digital security $(9 \mathrm{x})$ with one respondent having equal experience and one person having more experience in physical security.

We also gathered information about how participants evaluate the security of hybrid systems or design them. ISO 27001/27002 is slightly less relevant for this: it was mentioned five times for integrated analysis and three times for integrated design. This is an indication that existing methods pay limited attention to hybrid systems and their design.

Finally, participants rated the effort that is spent on physical security. On average that was $27 \%$ of the total number, with lows of zero and a high of $80 \%$. This appears to be a high number, which could be due to self-selection: only persons with an interest in physical security would participate. (We could not find any connection with the sector in with the participants were active.)

Summarizing, participants had overall more experience with digital security compared to physical security, and also spend more time on it. ISO 27001/27002 is not as dominant for security assessments and designs as could be expected of the de facto security standard. Especially it does not play an important role in evaluating the security of hybrid systems or in designing them.

\subsubsection{Method 1: System Analysis}

This section discusses the utility and usefulness of the first method (question FGRQ2). 


\section{Identifying Properties in Entities}

To begin, participants were asked to identify properties in entities. Figure 12.2 shows the model answers for each entity and property. These answers have been constructed by the author by applying the method to the example.

\begin{tabular}{llllll}
\hline Property & Central & Card & Chip & Chip & Bus, \\
& $\begin{array}{l}\text { Processing } \\
\text { System }\end{array}$ & Reader & Card & & $\begin{array}{l}\text { Tram, } \\
\text { Metro }\end{array}$ \\
\hline Active & false & false & false & false & true \\
Cohesive & false & true & true & true & true \\
Decayling & false & false & true & false & true \\
Dense & false & true & true & true & false \\
Difficultly manufacturable & true & true & true & true & true \\
Easily manufacturable & false & false & false & false & false \\
Enclosed with opening & false & false & false & false & true \\
Identifiable & possible & true & true & true & true \\
Inert & true & true & true & true & false \\
Invisible & false & false & false & false & false \\
Large & true & true & false & false & true \\
Limited spacious & false & false & false & false & true \\
Modifiable & possible & false & false & false & true \\
Movable & false & false & true & true & true \\
Slow & possible & false & false & false & false \\
Sound producing & false & true & false & false & true \\
Strong & possible & true & false & false & true \\
Uncopyable & true & possible & true & true & false \\
Visible & possible & true & true & true & true \\
Weak & possible & false & true & true & false \\
\hline & & & & & \\
& & & & & \\
& & & & &
\end{tabular}

Figure 12.2: Model answers for identifying properties in entities. "True" indicates that the entity has the property, "False" that it has not. "Possible" indicates that the property can be both present and not present, depending on the particular system that the participant is considering.

Figure 12.3 shows the correctness percentages for each property and entity with respect to these model answers. A '-' indicates that this property is excluded as both answers can considered to be correct. The last column indicates the correctness percentages for a specific property, where the number of participants is not weighted. The bottom row sums up the percentages for each entity. 


\begin{tabular}{|c|c|c|c|c|c|c|}
\hline Property & $\begin{array}{l}\text { Central } \\
\text { Processing } \\
\text { System }\end{array}$ & $\begin{array}{l}\text { Card } \\
\text { Reader }\end{array}$ & $\begin{array}{l}\text { Chip } \\
\text { Card }\end{array}$ & Chip & $\begin{array}{l}\text { Bus, } \\
\text { Tram, } \\
\text { Metro }\end{array}$ & $\begin{array}{l}\text { Unweighted } \\
\text { Average }\end{array}$ \\
\hline Active & 100 & 75 & 22 & 50 & 0 & 49 \\
\hline Cohesive & 100 & 63 & 33 & 50 & 0 & 49 \\
\hline Decayling & 100 & 88 & 78 & 50 & 100 & 83 \\
\hline Dense & 100 & 38 & 56 & 50 & 0 & 49 \\
\hline $\begin{array}{l}\text { Difficultly } \\
\text { manufac- } \\
\text { turable }\end{array}$ & 100 & 88 & 67 & 100 & 0 & 71 \\
\hline $\begin{array}{l}\text { Easily manu- } \\
\text { facturable }\end{array}$ & 100 & 88 & 78 & 100 & 100 & 93 \\
\hline $\begin{array}{l}\text { Enclosed with } \\
\text { opening }\end{array}$ & 100 & 88 & 89 & 100 & 0 & 75 \\
\hline Identifiable & - & 63 & 89 & 100 & 100 & 88 \\
\hline Inert & 100 & 88 & 0 & 0 & 100 & 58 \\
\hline Invisible & 100 & 100 & 100 & 100 & 100 & 100 \\
\hline Large & 100 & 88 & 89 & 100 & 0 & 75 \\
\hline $\begin{array}{l}\text { Limited } \\
\text { spacious }\end{array}$ & 50 & 75 & 67 & 100 & 0 & 58 \\
\hline Modifiable & - & 63 & 33 & 50 & 0 & 37 \\
\hline Movable & 100 & 88 & 89 & 100 & 0 & 75 \\
\hline Slow & - & 88 & 44 & 50 & 100 & 71 \\
\hline $\begin{array}{l}\text { Sound } \\
\text { producing }\end{array}$ & 100 & 63 & 89 & 100 & 100 & 90 \\
\hline Strong & - & 88 & 100 & 100 & 100 & 97 \\
\hline Uncopyable & 100 & - & 22 & 50 & 100 & 68 \\
\hline Visible & - & 100 & 89 & 50 & 0 & 60 \\
\hline Weak & - & 100 & 67 & 50 & 100 & 79 \\
\hline $\begin{array}{l}\text { Unweighted } \\
\text { Average }\end{array}$ & 96 & 81 & 65 & 73 & 50 & 71 \\
\hline
\end{tabular}

Figure 12.3: Correctness percentages for identifying properties. - indicates that the property is excluded as both answers can be correct. The Unweighted Average column shows the correctness percentages for a specific property, where the number of participants is not weighted. The bottom row sums up the percentages for each entity.

The numbers vary for each property, but we conclude that overall, in a large majority of the cases, participants were able to detect the properties correctly in the entities. Non-response is not distinctly observable in this figure, because it is the same as not identifying a property in an entity. 


\section{Identifying Entities and Properties}

Next, participants had to identify two physical entities in the system, in which all participants succeeded. For each of these entities, they indicated which physical security properties were applicable to them, based on a short explanation of the properties. After normalization the following entities were distilled:

- Central processing system $(2 \mathrm{x})$

- Card reader (8x)

- Chipcard (9x)

- Chip (2x)

- Bus/train/metro (1x)

We checked the identified properties against the correct values. As the correctness of the answers depends on their particular line of reasoning (which is not fully written down in this case) there is no completely accurate model answer set. For each property and entity we determine if the presence of the property was present, possibly present or not present. This in turn allowed us to score the answers on a scale of false, possible, true.

\section{Attributing Properties to Entities and Investigating Changes}

Next we asked the participants to identify an entity on which the property 'difficultly to manufacture' was applicable. All the given answers were correct, with one respondent not answering the questions, and one respondent given an answer twice.

Following this, the participants had to investigate how this property could change (more difficult or more easy to manufacture) and what the impact of that on security could be. First, respondents gave several answers as to why their entity would be more difficult to manufacture. All these answers were correct, with three users not answering the question. (Figure 12.4 shows the aggregated answers.)

Figure 12.5 shows the impact that a change of the property has on security goals. (The model answers are the same for all entities.)

We also inquired after the view of the participants regarding the usability of the method: ten out of eleven participants felt capable to identify relevant physical (and digital) security properties in real-life systems.

As for utility, five people believed the integrated risk assessment method could yield more accurate results than earlier approaches; four respondents did not know and two thought it could not. 


\begin{tabular}{llll}
\hline Entity & Count & $\begin{array}{l}\text { More difficult to } \\
\text { manufacture }\end{array}$ & Less difficult to manufacture \\
\hline Smart card & $8 \mathrm{x}$ & $\begin{array}{l}\text { use special chip } \\
\text { put (more) photos } \\
\text { change card size }\end{array}$ & $\begin{array}{l}\text { use mainstream technology } \\
\text { use non-electronic card (barcode) } \\
\text { use anonymous card } \\
\text { use same as chipcard or chipknip } \\
\text { use standard chip or SD card }\end{array}$ \\
Chip & $1 \mathrm{x}$ & use unique key & $\begin{array}{l}\text { use redundant chip } \\
\text { use standard components }\end{array}$ \\
$\begin{array}{lll}\text { Card reader } \\
\text { Network }\end{array}$ & $1 \mathrm{x}$ & - &
\end{tabular}

Figure 12.4: Difficult entities to manufacture and ways to change this property.

\begin{tabular}{llllll}
\hline Manufacture Change & Impact & Model Answer & Correct & Incorrect & No Answer \\
\hline More difficult & Confidentiality & $>$ or $=$ & $5 \mathrm{x}$ & $0 \mathrm{x}$ & $6 \mathrm{x}$ \\
,,, & Integrity & $<$ & $5 \mathrm{x}$ & $0 \mathrm{x}$ & $6 \mathrm{x}$ \\
,,, & Availability & $<$ & $4 \mathrm{x}$ & $1 \mathrm{x}$ & $6 \mathrm{x}$ \\
Less difficult & Confidentiality & $<$ & $7 \mathrm{x}$ & $1 \mathrm{x}$ & $3 \mathrm{x}$ \\
,,, & Integrity* & $<$ & $6 \mathrm{x}$ & $0 \mathrm{x}$ & $5 \mathrm{x}$ \\
,,, & Availability** & $>$ or $=$ or & $6 \mathrm{x}$ & $0 \mathrm{x}$ & $5 \mathrm{x}$ \\
\hline
\end{tabular}

Figure 12.5: Impact on security goals when the property difficultly manufacturable changes. *) Answer $>$ with explanation also correct.) ${ }^{* *}$ ) Answer $<$ with explanation also correct.

\section{Conclusions about Utility and Usability}

Utility From the previous section, we conclude that about half of the participants had the opinion that the method could improve information security; only two persons believed it could not.

Usability As for the learnability component, participants were able to apply the methods after a short training time of about five minutes per exercise. The efficiency was similarly good, most exercises were concluded within five minutes. Regarding errors, from the correctness scores, we conclude that the participants were in most cases able to correctly identify properties and made a limited number of errors. However there was a significant amount of non-response, as was shown in the last answer. This can indicate that the respondents needed additional time or did not fully understand the question. (They did not reach out for help during the meeting.) As for the satisfaction component, the overall majority felt capable of applying the methods. 


\subsubsection{Method 2: System Patterns}

This section discusses the utility and usefulness of the second method (question FGRQ2). The evaluation of this method consisted of a simpler setup than the first method. We presented the users with the aforementioned OV chipcard system. Concerning the application of patterns, we explained three specific security problems of the system. We then asked for each of these problems to identify patterns to improve the situation. Next, participants had to identify a security pattern used in a feature of the system. The answers were scored slightly different this time: we identified the correct answers, and counted the correct and incorrect answers across all participants. For example the participants proposed a total of 15 patterns, of which 8 were correct, leading to a score of 53 percent. We conclude that the participants were able to use patterns more than $50 \%$ of the time correctly, while they could identify a pattern more than $90 \%$ of the time (Figure 12.6). Nonresponse is visible as a case where a participant does not fill in any pattern and is excluded from the correctness percentages.

\begin{tabular}{lllll}
\hline$\#$ & Solve problem I & Solve problem II & Solve problem III & Identify \\
\hline 1 & 4 & 1 & 11,12 & 2,3 \\
2 & $2,10,11,14$ & $4,5,11,12$ & 2,12 & 2 \\
3 & 11 & 9,12 & 12 & 2 \\
4 & 11 & 12 & 11,3 & \\
5 & 11 & 12 & 4 & 2,3 \\
6 & 11 & 4 & 4 & \\
7 & 11 & 12 & 11 & 2,3 \\
8 & 3 & $4,9,11,12$ & 4 & 3 \\
9 & 8,2 & 11,12 & 12,4 & 3 \\
10 & 12 & 3 & 7 & 2 \\
11 & 3 & & $3,7,11$ & $2,3,7$ \\
Model Answers & $2,7,11,13$ & $4,11,12$ & $4,11,13$ & 2,3 \\
Correctness \% & 53 & 67 & 47 & 93 \\
\hline
\end{tabular}

Figure 12.6: Results for patterns. The numbers correspond to the pattern IDs. For example participant \#1 proposed patterns 11 and 12 to solve problem III. The correctness percentage is the total number of correct answers divided by the total answers. (For example in column "Identify" there are 15 correct answers out of a total of 16 .

We also inquired after the view of the participants regarding usability and utility:

- Nine persons had the opinion that the library of integrated security patterns could lead to more secure systems, implying that the method had utility. 
- Nine respondents felt capable of designing systems that use these security properties, implying that the method was usable.

\section{Conclusions about Utility and Usability}

Utility The overall majority of the participants concluded that applying the method could lead to more secure systems.

Usability Similar to the first method, we conclude good results regarding learnability and efficiency, with very limited time spent on learning and executing the exercises. As for errors: we observed that the score for solving problem I-III range from 47-67 percent. This is a relatively low score, apparently it was difficult for the participants to find patterns to improve a system design. However identifying patterns in existing systems was relatively easy, with a 93 percent correctness score. We consider three possible explanations for this difference: first, the difference can be caused by challenges that the participants encountered for the first three exercises, for example that the explanation of the patterns were not clear enough to apply them. Second, another possible explanation is that designing systems in inherently more difficult than identifying parts of an existing system. Third, it could be that the first three exercises were just more difficult, but that there is no inherent difference. (A new focus group meeting could be able to answer this.) Finally, regarding satisfaction, most people felt capable of designing systems that use a mixture of physical and digital properties.

\subsubsection{Changes of Opinion and General Utility}

Participants hold relatively steady view on the benefits of physical and integrated security methods. Initially, four out of eleven participants believed that more emphasis on physical security could improve overall security. After being present in the focus group, five people believed more emphasis on physical security could lead to better secured systems.

Nine participants initially believed that integrated methods could help to improve security. At the end of the meeting, ten participants had the opinion that integrated security could yield more secured systems.

Regarding the overall utility of both methods: asked about organizations and systems that could benefit most from the results, the participants' answers included security consultants, governments, financial organizations, military, engineering companies, manufacturers. Specific target systems included Industrial Control Systems (ICS). 


\subsubsection{Opportunities for Improvements}

We explicitly asked the participants for ways to improve the methods (question FGRQ3). Specific recommendations included the following:

- Extend the system with business goals.

- Develop a more visual approach.

- Use stricter definitions of properties and risks.

- Include system scope assessment.

- Include humans in the design.

A number of these recommendations can considered to be out of scope. for example we chose to focus on physical and digital properties and less on the social domain. Also the approach is specifically focused on the three main security goals of confidentiality, integrity and availability. Systems like KAOS can link these to business goals (and indeed KAOS was used in the case studies), but it is not part of the two methods. Others, particularly the ideas to develop a more visual approach and more stricter definitions should be investigated and could likely lead to improvements.

\subsection{Discussion and Conclusions}

In this chapter, the results of the focus group meeting were discussed. The main objective of the focus group meeting was to evaluate the usefulness of the results and specifically the two methods, which was defined and operationalized as utility and usability. Usability was further split in learnability, efficiency, errors and satisfaction. Based on the analysis presented, specifically on the results of the subgoals and the results for utility, we conclude that the participants found the method useful for their purpose.

\subsubsection{Validity of the Results}

We will now consider the validity of the results, using the four types of validity mentioned in Section 5.13 (construct, conclusion, internal and external validity). We first consider the impact of non-response, as a general threat to the validity of the results.

\section{Non-Response}

Non-response in the focus group meeting falls into two categories. 
- Non-response on general questions. An example of such a question is: "How many years of professional experience do you have in the area of information security?";

- Non-response on questions that relate to execution of the methods.

First, non-response on general questions is almost not present, so there are no threats to the validity of the results for these questions. Second, regarding the execution of the methods, we observed a significant amount of non-response for specific questions. We interpreted this as a situation where the participants were unable to formulate the correct answer, and/or potentially had insufficient time. As this type of non-response is clearly observable, it weighed into the conclusions that were reached and does not impact the validity.

In other cases non-response was not directly ore more difficultly observable, as it could not be separated from a conscious decision of a participant that reached a negative conclusion. The effects of the non-response are varied: as a first example we consider Figure 12.3 As both negative and positive answers could potentially be correct there is a potential negative effect on the validity of the conclusions reached, which could either overestimate or underestimate the correctness percentages. (The number of true and false model answers are almost equal, 45 against 48 .)

As a second example we consider Figure 12.6 In the last column the patterns identified by the participants are listed. Those participants that did not find any pattern were counted as non-response. These could also have been counted as errors, and thus lowering the correctness scores. (Two out of eleven participants did not fill in any pattern.)

\section{Construct Validity}

Construct validity concerns justification of the conceptual framework and the constructs that were measured or identified, and whether we really measured the constructs of our framework. The main construct in this chapter was the definition of usefulness, consisting of utility and usability, and the breakdown of usability into the quality aspects of learnability, efficiency, errors and satisfaction. These constructs, originating in theory on web design, represent a logical breakdown of usefulness and are widely used in practice. As such we consider these constructs to be appropriate for evaluating any design, including a design for a risk assessment method.

\section{Conclusion Validity}

Conclusion validity concerns whether the conclusions reached are supported by evidence, meaning that can be traced back to the evidence through a sequence 
of valid reasoning steps. Conclusions can be traced back to two types of information:

- The answers given by participants in the questionnaire

- The metadata about the focus group meeting (for example how participants were found and the duration of the focus group meeting).

Concerning the first types of information, the answers are given in Appendix A, Section 12.2 and 12.3 explain in detail how the conclusions were derived. Considering these, we conclude that there is almost complete traceability of the conclusions.

\section{Internal Validity}

Internal validity concerns the validity of causal explanations of the observations and whether alternative explanations are possible. In the focus group, we have not directly investigated a causal relation between two variables. However, we are interested in understanding one causal relation: does the application of the artifacts (the methods) increase or decrease information security? Based on the evidence generated from the focus group, we conclude that this effect is likely, although more evidence would be needed to achieve more certainty about this conclusion. We have no evidence that there were other artifacts introduced in the focus group meeting that could have caused this result.

\section{External Validity}

External validity concerns the issue whether the results can be generalized to other cases. For the focus group meeting, this concerns the issue whether the results obtained through the participants hold for the larger population of security experts. We used convenience sampling for the focus group: we simply used all the means available to find participants. This means that no statistical analysis is possible and neither can we perform a statistical generalization to the entire population.

A specific issue is that participants selected themselves: the participants were security experts with an interested in participating in a focus group meeting on physical and digital security. As such they do not necessarily represent the population of security experts but rather a subset of it. This would specifically impact the utility of the results, as ordinary security professionals might not be interested or able to use the results. However the number of participants that thought more emphasis on physical security was needed was limited (four at the beginning of the meeting and five after the exercises) so we expect this effect to be limited.

As for usability, we do not see that there should be a specific impact for usability as the methods do not need specific expertise in the area of physical security. 
Overall we conclude that the results, given the fact that they were positive, are a strong indication of both the utility and the usefulness in other cases too.

This provides sufficient reason to do the experiment again, and investigate if we can generalize across different focus groups (lab to lab generalization) or perform an actual field test, to see how the results hold out in practice.

\subsubsection{Lessons Learned}

Overall, the focus group was found to provide useful information for the testing of the usefulness of the methods. Within a short period, we gathered a substantial amount of information about how the participants applied them. In addition to that, participants suggested many ways to improve on the methods, which can be used to make them even more useful. 


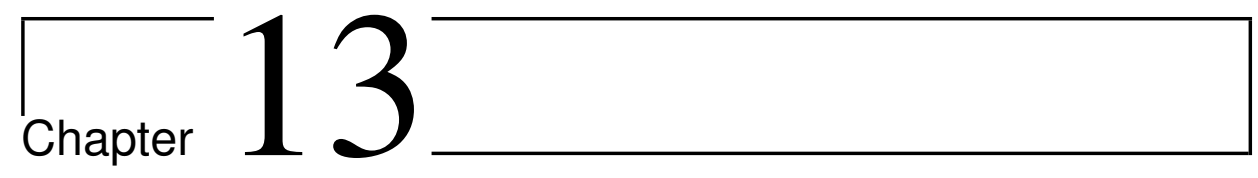

\section{Conclusions}

\subsection{Introduction}

In this final chapter we summarize the findings, reflect upon them and provide an outlook at current developments and present directions for future research.

\subsection{Summary of Findings}

Chapter 1 listed the six research questions, and we briefly list the answers to each of them.

RQ1: What are the properties of physical entities that physical security mechanisms depend upon?

In total we found twenty physical properties as listed in Section 10.3 . These were divided in five clusters of properties: the Architectural, Audible, Independence, Material and the Visual cluster.

RQ2: What are the properties of digital entities that digital security mechanisms depend upon?

In total we found five properties as listed in Section 10.4. These were divided in three clusters: Complexity, Speed and Accuracy.

RQ3: What are the key differences between physical and digital security mechanisms?

The main differences are listed in Section 10.5. Physical and digital security are realized (and threatened) in entirely different ways, and physical security appears to be more refined as there are significantly more physical properties than digital properties. 
RQ4: How can physical and digital security mechanisms be combined?

In total we found twelve different types of hybrid systems in the case studies (Figure 10.6). We used these to create a taxonomy in Section 10.7, with three main branches: pure hybrid systems, physicalized systems (that use physical protection mechanisms) and digitalized systems (that use digital protection mechanisms).

RQ5: What are the trade-offs between physical and digital mechanisms?

The trade-offs are listed in Section 10.7. From the data we conclude that analytically the confidentiality of a system decreases as it becomes more digital. The same holds true for availability but in a later stage, as hybrid systems still have good availability. Integrity in a hybrid system is higher than in a pure physical or digital system.

RQ6: How can we apply the knowledge about physical and digital properties, combinations and trade-offs to improve security in an integrated way?

We presented two methods to apply the results of the thesis: one method for assessing risks and one set of security patterns for creating hybrid systems (Section 11.4). These were validated using a focus group meeting in Chapter 12

\subsection{Reflection}

\subsubsection{Reflection on Systems and Their Context}

In this section we reflect on the role of the context and how it affects realization of security goals. Figure 13.1 lists the different systems we examined.

One major difference is the typical working process in the different case studies in terms of workload and duration. In general: the higher the workload and the longer the duration, the more difficult it is to secure.

- For access control and virtualization, the transaction volume is high. These systems are designed to be used by entire corporations or countries: no one is expecting that a payment provider would process her payments by hand because they should remain confidential. By contrast, paper voting is completely feasible, as each person delivers at most one ballot per election and the expectation that someone would count these ballots by hand is not unreasonable.

- The process for access control and virtualization is open-ended whereas it is finite for paper voting: the election has a clear beginning and ending point. For rights management systems, there are different cycles: for a stadium concert, the process lasts at most several months, from initial ticket sales to 


\begin{tabular}{|c|c|c|c|c|}
\hline & Access Control & Voting & IT Infrastructure & Rights Management \\
\hline Physical & Keys, locks & Paper voting & $\begin{array}{l}\text { Non-virtualized } \\
\text { Infrastructures }\end{array}$ & $\begin{array}{l}\text { Book, Live music, } \\
\text { Tape recorder, Radio } \\
\text { broadcast, Board game, } \\
\text { Live match, Video } \\
\text { recorder, TV broadcast }\end{array}$ \\
\hline Digital & $\begin{array}{l}\text { Logical access } \\
\text { control }\end{array}$ & $\begin{array}{l}\text { Electronic } \\
\text { voting }\end{array}$ & $\begin{array}{l}\text { Virtualized } \\
\text { Infrastructures }\end{array}$ & $\begin{array}{l}\text { PDF, Internet radio, PC } \\
\text { and MP3 player, PC } \\
\text { game, Live stream, } \\
\text { Video-sharing site }\end{array}$ \\
\hline Hybrid & $\begin{array}{l}\text { Location-based } \\
\text { access control }\end{array}$ & $\begin{array}{l}\text { Ballot printer } \\
\text { Optical Scan } \\
\text { Machine } \\
\text { Redundant } \\
\text { system } \\
\text { Scantegrity II } \\
\text { Pen system }\end{array}$ & $\begin{array}{l}\text { Partially virtualized } \\
\text { infrastructures }\end{array}$ & $\begin{array}{l}\text { E-reader and e-book, } \\
\text { Portable media player, } \\
\text { Online console game, } \\
\text { Pay-per-view }\end{array}$ \\
\hline
\end{tabular}

Figure 13.1: Overview of systems.

the concert itself. Computer games have a life cycle of two to three years at most. For on-line music usage is open ended, music sold now can still be used decades later.

Concerning the physical context, our cases differ as to the physical security that is in place, and the number of people that are involved. Physical and locationbased access control require the presence of people, but not necessarily in a strictly secured environment. By contrast, virtualized infrastructures are located in tightly controlled data centers that function best with a minimum of people inside. Voting procedures take place in a polling station that is not physically secured at all. Rights management solutions differ: for stadiums physical access control is in place, whereas game consoles are stored in private at users' homes. Access control and virtualized infrastructures are typically under the control of enterprises, whereas voting systems are controlled by governments and citizens, and RM systems by corporations and end-users.

Another major difference is the tolerance for errors and attacks. In rights management systems, one security weakness leading to content being freely available can render it useless for that content because it will be freely available on the Internet. In voting systems, one fraud case involving several hundreds of votes is not necessarily detrimental to its goals, as the results depend on millions of votes. For a physical access control system the effects entirely depend on the type of keys being used and stolen; theft of the master key can have a great impact, whereas a normal key loss may even go unnoticed. In this thesis, we implicitly aggregated probabilities and impacts into positive and negative effects, but these are clearly 
a simplification. As already mentioned in Chapter 5 we believe that our results (especially the properties and patterns) are helpful in constructing more refined probabilistic models.

\subsubsection{Reflection on Broad-range Theories}

In Chapter 3 we presented four broad-range theories about automation and its impact. We will now reflect on these, placing the result from our case studies into context. First, Sieghard [194] and Floridi [58] predicted that automation causes a decrease in the confidentiality of information. Especially with respect to confidentiality, these predictions are confirmed by our results (Figure 10.11). Blakley [21] viewed physical security as having inherent, and digital security as having imposed properties. Concerning the first, we have indeed found inherent properties of physical and digital entities. Although based on different principles the digital characteristics seem similarly inherent as their physical counterparts. Finally Lessig held the position that cyberspace is governed by code and its properties are determined by software programs - which governments can ultimately adapt and mandate as they see fit. We believe this is true to some extent, but copyability seems a fixed characteristic for now; the problems of rights management are more likely to be solved by implicit mechanisms (Section 10.7) than by explicit methods such as TPM.

\subsubsection{Reflection on Completely Automated Systems}

A recurrent theme is the security of completely automated systems: what happens to security if a system is fully automated? Our evidence is very conclusive about their security: they are less secure than hybrid systems. Vice versa, the security of completely automated systems is increased by adding physical security mechanisms.

For access control, completely automated systems are subject to access from everywhere. Physical constraints, such as location-based access control can improve their security. For voting, we have seen that completely electronic systems have a low assurance rate concerning the confidentiality and integrity goals. Only if we drop confidentiality we can be assured of the system's integrity. As for IT infrastructures, we observed that properties such as uniqueness, locationboundedness and monotonicity are threatened when systems are completely virtualized. Rights management of purely digital files, which do not depend on any physical entities is impossible. Each security mechanism to protect content has a physical basis. 


\subsubsection{Reflection on Case Study Approach}

The overarching method in the thesis was a multiple case study approach, where we investigated the security of physical, digital and hybrid systems across four domains. With the results having been presented, we can reflect on the approach. For this reflection, we choose to ways:

1. Explain pitfalls and challenges in executing the research.

2. Contrast the approach with a complete alternative one, namely to study checklists and security standards regarding physical and digital security. (for example ISO 27002, PCI-DSS, DHS etc. etc.).

(We will not discuss the validity of actual outcomes in detail, because this was extensively evaluated in Section 10.8 .)

\section{Difficulties in the Approach}

Depth of the Case Studies and Effort Involved As we studied so many systems, a very large amount of literature had to be studied to learn about existing system designs and risks. In addition to that, actual on-site visits and interviews took place. This approach resulted in deep case studies that took a long time to develop and report on. Even though a large amount of data was collected, we would still have preferred to include more actual site visits than were done, specifically to have direct access to practitioners with domain expertise. This proved to be difficult, as several organizations that were approached declined to participate or did not have actual data available that they were able to share.

Data Processing The study of all these systems resulted in an enormous amount of data. Investigating this data, harmonizing and checking it proved to be challenging. In the end data was loaded into a special database, which allowed consistency checks with the data and automatically generating the main tables that contained the results. Loading data, programming the scripts and validating the results proved to be a very labor intensive process.

\section{Alternative Approach}

A completely alternative approach would have been to study what are seen best practices, such as ISO 27001/27002 checklists. These are also concerned with physical and digital security, and codify knowledge about threats and mitigations in these domains. Through this study, we could also derive mechanisms about the workings of digital and physical security. This approach would have had three drawbacks compared to the approach taken: 
first, the consequence would have been that combinations of digital and physical security would not have been included, because hybrid systems designs and security are not considered in general checklists. A complimentary approach could be the study of a specific class of hybrid systems, such as industrial control systems (commonly known as ICS or SCADA systems), and the checklists that are used in that domain. However, this would make it difficult to generalize the data to other types of hybrid systems. Second, the results would have been based on common industry best practices, which are often outdated compared to what experts know today. Third, the results would not be grounded as much in actual systems. The advantage of the chosen case study approach is that there is a relatively tight integration with actual systems and their security; this would not have been the case had we studied checklists.

Advantages of checklist investigation would be that the results are more easily understood by practitioners, as they are derived from explicit knowledge that they already apply. Furthermore, this would have been a relatively quick approach because the checklists are readily available. The time saved could then be used for further validation or experimentation.

Overall we conclude that a faster approach would have been possible, which would have resulted in less data that would have to be complimented by other means to achieve the same level of depth as in the case study approach.

\subsection{Outlook}

Ongoing technological developments weaken properties that we found to be the cornerstones of physical security. We explain this for three clusters of properties: the visual, independence and material cluster. Concerning the visual cluster, we explained before that limited visibility enables security. However, limited visibility is threatened:

- New devices enable us to see too much. Cameras and image processing software are becoming better and these enable us to take pictures of locks (Chapter 6), or observe the typing passwords from a distance with unprecedented precision. It cannot be expected that devices will reduce their capabilities, in fact their capabilities will increase. Methods that are proposed to limit capabilities (such as disabling cameras in sensitive locations [188]) will likely not be realized in practice because the cameras are under the direct control of the users, and enforcement would require unprecedented collaboration from vendors.

- Devices such as cameras are being miniaturized and can be hidden almost anywhere. This makes it increasingly hard to be certain of the absence of cameras as persons can no longer identify them visually. Thus security 
mechanisms that depend on limited visibility and personal inspection are becoming outdated: we require specific devices to assess the absence of other devices. Already smartphones can pose problems with voting processes (Chapter7) and movies played in cinemas can be recorded with simple digital cameras (Chapter 9).

The independence cluster, specifically the inertness property plays such an important role in assuring the confidentiality and integrity of systems is under threat as well. With the coming Internet of Things [85], soon there will be no "trusted third space" any more, which is not under some form of control by an agent. Technologies such as RFID chips require little or no power, and can be embedded everywhere, communicating with each other and sensing their environment. This "activates" existing inert material: paper with RFID chips suddenly becomes capable of storing, processing and communicating information. Thus a technology as RFID can threaten the natural confidentiality that exists in the physical world. Other examples include USB connections that integrate the power connection with data communication and wireless charging methods. They make it very hard to ensure that a device is indeed switched off and does not transmit data.

Regarding the material cluster: paper voting depends on the inability of persons to duplicate key voting material, access control on the impossibility of duplicating persons and keys, IT infrastructure on the inability to duplicate cables and servers. The long trend of increasing manufacturing capabilities is eroding the limits of manufacturing. For example key duplication does not require specialized equipment, as keys can simply be printed [163]. Likewise copyright on physical entities becomes more difficult to enforce as everyone can scan and recreate items. $3 \mathrm{D}$ printers such as the RepRap ${ }^{1}$ are still in their early stages of development, but will become cheaper and more powerful over time.

The implications of these developments are that physical security as currently in use will likely cease to work as it does now: the future will paradoxically require digital mechanisms to ensure physical properties. For example the absence of devices in a location will only be assured by using high-tech scanners, and 3D printers might be subjected to tight regulation to prevent undesired objects appearing [50].

\subsection{Future Work}

In this section we offer promising directions for further research that so far we were incapable of undertaking yet.

1 http://reprap.org 
Formalization The properties of physical and digital entities are relevant and useful for formal modeling. As so far no such formal model has been built that incorporates all these properties, we believe this is a very promising research direction.

A good test case for such a model would be finance. Not unlike content producers, central banks are facing counterfeiters who are forging banknotes, taking all sorts of preventive and detective mechanisms such as watermarking and auditing. Furthermore, cash comes in both purely digital form (a bank account), physical (bank account) and hybrid forms on smart cards ${ }^{2}$

Simulations In Chapter 3 we outlined three conceptual strategies for the reintroduction of physical processes, which could potentially improve information security.

1. de-automation: making automated systems physical again

2. recombination: combining physical and digital mechanisms

3. simulation: simulating physical systems in IT

This thesis has primarily investigated recombination, and it has been found a good solution for improving security. De-automation is a very strong measure but has been applied in practice, for example for the Dutch and German elections (Chapter 7). Simulation remains to be investigated.

Voting Systems In the section on applications, we outlined three strategies for governments to ensure voting security, using existing technologies. As future work, we believe that a fourth option can be developed based on the pattern of "physical automation" from Section 10.6. With physical automation we mean the usage of conveyor belts, robots and other industrial equipment that can be used to reorganize the voting process. Citizens would still be using paper, but the actions after the ballot deposit, including transportation and counting, would be carefully scripted and automated. Such automation would greatly improve the integrity and assurance of the voting process and eliminate many human errors. In addition, the visibility of the process would not be limited (it could even be enhanced, because the process is standardized), and the paper would still be inert. To ensure adequate confidentiality, ballots could even be shredded on-site, automatically after counting them.

\footnotetext{
${ }^{2}$ Even location-based access control is already applied, as the Dutch Rabobank blocks pin transactions from outside of Europe by default.
} 
Potential for New Mechanisms A final research direction is to simulate physical properties in IT. Our research shows the general tendencies of IT systems to be loss of location boundedness, uniqueness and monotonicity, which are core traits of physical security mechanisms. We are interested in IT-based methods that can somehow recreate these properties inside IT, simulating for example the slowness of physical transportation.

\subsection{Conclusion}

De-perimeterization is an ongoing problem that negatively impacts information security. The examination of this phenomenon led us to investigate the security-relevant differences between physical and digital security mechanisms and the potential of combinations. Our results indicate that the outcomes depend on context and specific security goals but clearly hybrid security mechanisms are to be preferred over digital mechanisms. We hope that the conclusions will be used to improve the state of information security, as well as the research thereof. 


\section{Part IV}

\section{Appendix}





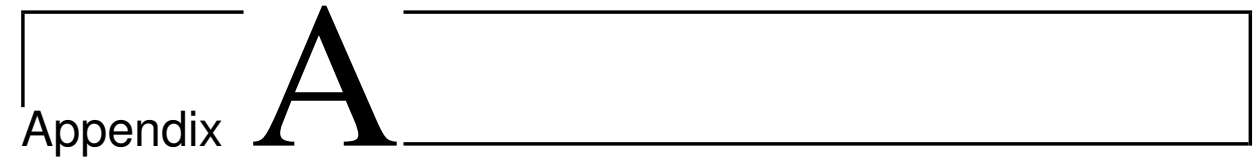

\section{Focus Group Meeting Material}

This appendix presents material used in the focus group meeting. Originally printed on A4, the layout has been modified to fit this appendix. 


\section{A.1 Questionnaire}

\section{Survey}

\# Question

Answer

Part I (Background, experience and views)

1. What type of organization do 0 IT Industry

you work for?

0 Academia

(Select one or more)

0 Government

0 Non-profit

0 Other ...

2. Which position do you hold in this organization?

3. How many years of professional experience do you have in the area of information security?

4. How would you currently perform an assessment of physical security? (Select one or more) ....... years How would you currently perform an assessment of digital security? (Select one or more)

6. In which field is your experience greater, in physical or in digital security mechanisms?

7. How would you perform an analysis of a system that uses both physical and digital security mechanisms? (Select one or more)

8. What method/approach would you use to design a system that uses both physical and digital security mechanisms? (Select one or more) 0 Generic risk assessment / threat analysis 0 ISO 27002 checklist 0 Own checklists 0 Other ... 0 Don't know 0 Generic risk assessment / threat analysis 0 ISO 27002 checklist 0 Own checklists 0 Other ... 0 Don't know 0 Physical security 0 Digital security 0 Equal experience

0 Generic risk assessment / threat analysis 0 ISO 27002 checklist

0 Own checklists

0 Other ... 0 Don't know

0 Generic risk assessment / threat analysis 0 Requirements engineering 0 ISO 27002 checklist 0 Own checklists 0 Other ... 0 Don't know 
9. For all systems that you have analyzed or designed, how much effort is put in physical security, if the total security effort is $100 \%$ ?

(Given an estimation as a percentage)

10. Could security be improved by putting more emphasis on physical security mechanisms?

... ... percent physical security

11. Could security be improved by tighter integrating physical and digital security mechanisms?

0 Yes

0 No

0 Don't know

0 Yes

0 No

0 Don't know

<< End of Survey >> 


\section{Exercises}

The Dutch OV Chip card system uses a contactless smartcard. Persons who use the public transport system must swipe their card when they enter or exit the system, for example when boarding or off boarding a bus. (Check-in or checkout) The chip card holds money and the amount stored on the card is updated during check-in and checkout. Check-in and checkout events are sent to a central processing system over a mobile network.

The next questions concern this system.

\section{Question 1 (Analysis Method)}

1.a list two physical entities used in this system

\begin{tabular}{|c|c|}
\hline Physical entity 1 & \\
\hline Physical entity 2 & (2) \\
\hline
\end{tabular}


1.b. Identify which physical security properties apply to these entities (as many as possible).

If a property applies to the entity, indicate this by placing a " $\mathrm{X}$ " in the cell.

\begin{tabular}{|c|c|c|c|c|}
\hline Property & Explanation & Example & $\begin{array}{l}\text { Physical } \\
\text { entity } 1\end{array}$ & $\begin{array}{l}\text { Physical } \\
\text { entity } 2\end{array}$ \\
\hline Active & Moving, action & Person & ……................ & (...................... \\
\hline Cohesive & Tightly fit together & Brick & ……................... & …..................... \\
\hline Decaying & Wearing down & Newspaper & …................... & ….................. \\
\hline Dense & No holes, cavities & Brick & …..................... & …................... \\
\hline $\begin{array}{l}\text { Difficultly } \\
\text { manufacturable }\end{array}$ & Hard to create & Banknote & & $\ldots$. \\
\hline $\begin{array}{l}\text { Easily } \\
\text { manufacturable }\end{array}$ & Easy to create & A4 paper & f........................ & f...................... \\
\hline $\begin{array}{l}\text { Enclosed with } \\
\text { opening }\end{array}$ & $\begin{array}{l}\text { Hollow object with } \\
\text { opening }\end{array}$ & Wine bottle & ………….... & …………..... \\
\hline Identifiable & Can be identified & Person & ...................... & ........................ \\
\hline Inert & Does not move & Brick & …….................. & ……................. \\
\hline Invisible & Cannot be seen & Radio wave & ....................... & ..................... \\
\hline Large & $\begin{array}{l}\text { Difficult to hide by } \\
\text { a person }\end{array}$ & Table & ………............ & ........................ \\
\hline $\begin{array}{l}\text { Limited } \\
\text { spacious }\end{array}$ & $\begin{array}{l}\text { Space in which } \\
\text { objects can still be } \\
\text { tracked }\end{array}$ & $\begin{array}{l}\text { Meeting } \\
\text { room }\end{array}$ & …………....... & …….............. \\
\hline Modifiable & Can be changed & Paper & . & . \\
\hline Movable & $\begin{array}{l}\text { Can be moved by a } \\
\text { person }\end{array}$ & Brick & . & ….................. \\
\hline Slow & $\begin{array}{l}\text { Can be intercepted } \\
\text { by a person }\end{array}$ & Person & . & ………......... \\
\hline $\begin{array}{l}\text { Sound } \\
\text { producing }\end{array}$ & Produces sound & Claxon & …..................... & ........................ \\
\hline Strong & Difficult to break & Brick wall & . & ……............... \\
\hline Uncopyable & Cannot be copied & Person & ………............. & ……................ \\
\hline Visible & Can be seen & Brick & …................... & 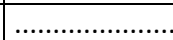 \\
\hline Weak & $\begin{array}{l}\text { Easy to break by a } \\
\text { person }\end{array}$ & Paper & f....................... & f....................... \\
\hline
\end{tabular}


Consider the following physical property with a hypothesis about security

\begin{tabular}{|l|l|}
\hline Property & Difficultly manufacturable \\
\hline Category & Manufacture \\
\hline Effect & Positive effect on confidentiality and integrity \\
\hline Explanation & $\begin{array}{l}\text { Manufacturing increases the chance of manipulating business } \\
\text { process to the attacker's advantage. When objects are difficult to } \\
\text { manufacture that increases the confidentiality and integrity. }\end{array}$ \\
\hline Conditions & Business process depends on limited and /or unique items. \\
\hline
\end{tabular}

1.c. On which entity is this property applicable? (Name one)

1.d. For the entity identified in $1 c$, can this property be changed, meaning that it becomes more difficult to manufacture or less difficult to manufacture? What would be the effect of that change?

\begin{tabular}{|c|c|c|}
\hline Change & If/How this could happen & Effect on security \\
\hline $\begin{array}{l}\text { More difficult to } \\
\text { manufacture }\end{array}$ & 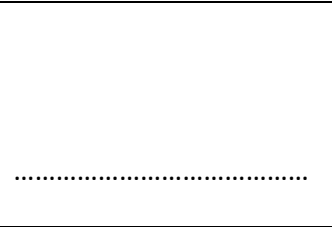 & $\begin{array}{l}\text { Confidentiality: } \\
\text { Integrity: } \\
\text { Availability: }\end{array}$ \\
\hline $\begin{array}{l}\text { Less difficult to } \\
\text { manufacture }\end{array}$ & & $\begin{array}{l}\text { Confidentiality: } \\
\text { Integrity: } \\
\text { Availability: }\end{array}$ \\
\hline
\end{tabular}




\section{Exercise 2: Design}

For the OV Chip card there are three specific security problems:

I. Users forget to check-in or checkout (integrity problem)

II. An attacker can put a nearly invisible sticker with a custom chip on the smartcard that can be charged by the attacker. When checking in, the custom chip is used rather than the original. (integrity problem)

III. Fraud can take place by copying the chip cards (confidentiality, integrity problem)

2.a. Using the security design patterns, suggest ways to change the system design to solve problem I.

2.b. Using the security design patterns, suggest ways to change the system design to solve problem II.

2.c. Using the security design patterns, suggest ways to change the system design to solve problem III.

Consider that at some point in time, users can order chip cards over the Internet, which are sent to their address by ordinary mail. This opens up opportunities of fraud, for example pranksters requesting cards on behalf of others. Therefore it is decided that users must pickup their card themselves at a railway station.

2.d. What pattern is applied in the new situation? 


\section{Evaluation}

\begin{tabular}{|c|c|c|}
\hline \# & Question & Answer \\
\hline 1. & $\begin{array}{l}\text { Do you feel capable of } \\
\text { identifying relevant physical } \\
\text { and digital security } \\
\text { properties in real-life } \\
\text { systems? } \\
\text { (explain your answer) }\end{array}$ & $\begin{array}{l}0 \text { Yes } \\
0 \text { No } \\
\text { O Don't know } \\
\text { Explanation: }\end{array}$ \\
\hline 2. & $\begin{array}{l}\text { Do you feel capable of } \\
\text { designing systems that use } \\
\text { physical and digital security } \\
\text { properties? } \\
\text { (explain your answer) }\end{array}$ & $\begin{array}{l}0 \text { Yes } \\
0 \text { No } \\
0 \text { Don't know } \\
\text { Explanation: }\end{array}$ \\
\hline 3. & $\begin{array}{l}\text { If you consider your past } \\
\text { experiences, could the } \\
\text { integrated risk assessment } \\
\text { method yield more accurate } \\
\text { results as compared to your } \\
\text { earlier approaches / } \\
\text { methods? } \\
\text { (explain your answer) }\end{array}$ & $\begin{array}{l}0 \text { Yes } \\
0 \text { No } \\
0 \text { Don't know } \\
\text { Explanation: }\end{array}$ \\
\hline 4. & $\begin{array}{l}\text { If you consider your past } \\
\text { experiences, could the } \\
\text { library of integrated security } \\
\text { design patterns yield more } \\
\text { secure systems as compared } \\
\text { to your earlier approaches / } \\
\text { methods? } \\
\text { (explain your answer) }\end{array}$ & $\begin{array}{l}0 \text { Yes } \\
0 \text { No } \\
0 \text { Don't know } \\
\text { Explanation: }\end{array}$ \\
\hline 5. & $\begin{array}{l}\text { Which type of organization / } \\
\text { system could benefit most } \\
\text { from this research? } \\
\text { (explain your answer) }\end{array}$ & 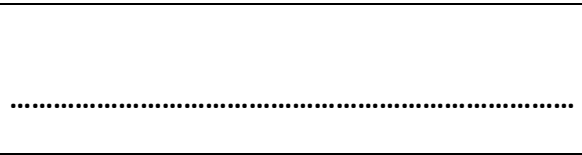 \\
\hline
\end{tabular}




\begin{tabular}{|c|c|c|}
\hline 6. & $\begin{array}{l}\text { After being present in the } \\
\text { focus group meeting, could } \\
\text { security be improved by } \\
\text { putting more emphasis on } \\
\text { physical security } \\
\text { mechanisms? }\end{array}$ & $\begin{array}{l}0 \text { Yes } \\
0 \text { No } \\
0 \text { Don't know }\end{array}$ \\
\hline 7. & $\begin{array}{l}\text { After being present in the } \\
\text { focus group meeting, could } \\
\text { security be improved by } \\
\text { tighter integrating physical } \\
\text { and digital security } \\
\text { mechanisms? }\end{array}$ & $\begin{array}{l}0 \text { Yes } \\
\text { O No } \\
\text { O Don't know }\end{array}$ \\
\hline 8. & $\begin{array}{l}\text { Do you have any } \\
\text { recommendation to improve } \\
\text { the integrated risk } \\
\text { assessment method? } \\
\text { (explain your answer) }\end{array}$ & 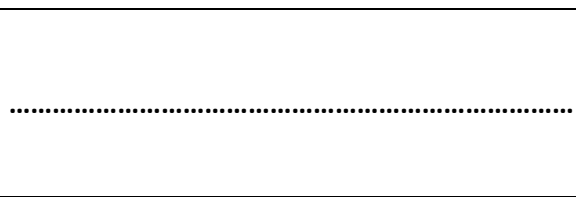 \\
\hline 9. & $\begin{array}{l}\text { Do you have any } \\
\text { recommendation to improve } \\
\text { the library of integrated } \\
\text { security design patterns? } \\
\text { (explain your answer) }\end{array}$ & 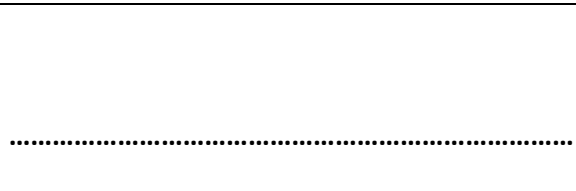 \\
\hline 10. & $\begin{array}{l}\text { Do you have any other } \\
\text { recommendations to } \\
\text { improve the focus group } \\
\text { meeting? } \\
\text { (explain your answer) }\end{array}$ & (1) \\
\hline 11. & $\begin{array}{l}\text { Would you like to receive } \\
\text { the complete methods } \\
\text { digitally? }\end{array}$ & $\begin{array}{l}0 \text { Yes } \\
\text { Name: } \\
0 \text { No }\end{array}$ \\
\hline
\end{tabular}

< End of Evaluation >> 


\section{A.2 Linkage between Focus Group Questions and Re- search Questions}

Figure A.1 explains how the questions contribute to the research questions of the survey.

\begin{tabular}{llll}
\hline Section & Question & Research Question & Details \\
\hline Survey & $1-9$ & FGRQ1: Background & - \\
& 10 & FGRQ2: Usefulness-Utility & Pre-test opinion physical security \\
& 11 & FGRQ2: Usefulness-Utility & Pre-test opinion more integration \\
Exercises & $1 \mathrm{a}-1 \mathrm{~d}$ & OB1: Usefulness-Usability & Method 1 \\
& $2 \mathrm{a}-2 \mathrm{~d}$ & OB2: Usefulness-Usability & Method 2 \\
Evaluation & 1 & OB2: Usefulness-Usability & Method 1 \\
& 2 & FGRQ2: Usefulness-Usability & Method 2 \\
& 3 & FGRQ2: Usefulness-Utility & Method 1 \\
& 4 & FGRQ2: Usefulness-Utility & Method 2 \\
& 5 & FGRQ2: Usefulness-Utility & General \\
& 6 & FGRQ2: Usefulness-Utility & Post test opinion physical security \\
& 7 & FGRQ2: Usefulness-Utility & Post test opinion more integration \\
& 8 & FGRQ3: Improvement & Method 1 \\
& 9 & FGRQ3: Improvement & Method 2 \\
& 10 & FGRQ3: Improvement & Focus group \\
& 11 & FGRQ1: Background & - \\
\hline
\end{tabular}

Figure A.1: Explanation of which survey questions yield information for which research questions (obtain background information, test usefulness in the form of utility and usability, and obtain recommendations for improvements).

\section{A.3 Answers for Method One}

Central Processing System Figure A.2 shows the results of the properties for the central processing system.

Card Reader Figure A.3 shows the results of the properties for the card reader.

Chipcard Figure A.4 shows the results of the properties for the chipcard.

Chip Figure A.5 shows the results of the properties for the chip.

Bus, Train, Metro Figure A.6 shows the results of the properties for the bus, tram or metro. 


\begin{tabular}{|c|c|c|c|c|}
\hline Property & $\# 1$ & $\# 2$ & Model Answer & Correctness $\%$ \\
\hline Active & & & false & $100 \%$ \\
\hline Cohesive & & & false & $100 \%$ \\
\hline Decaying & & & false & $100 \%$ \\
\hline Dense & & & false & $100 \%$ \\
\hline Difficultly manufacturable & $\mathrm{x}$ & $\mathrm{x}$ & true & $100 \%$ \\
\hline Easily manufacturable & & & false & $100 \%$ \\
\hline Enclosed with opening & & & false & $100 \%$ \\
\hline Identifiable & & $\mathrm{x}$ & possible & - \\
\hline Inert & $\mathrm{x}$ & $\mathrm{x}$ & true & $100 \%$ \\
\hline Invisible & & & false & $100 \%$ \\
\hline Large & $\mathrm{x}$ & $\mathrm{x}$ & true & $100 \%$ \\
\hline Limited spacious & & $\mathrm{x}$ & false & $50 \%$ \\
\hline Modifiable & & $\mathrm{x}$ & possible & - \\
\hline Movable & & & false & $100 \%$ \\
\hline Slow & & & possible & - \\
\hline Sound producing & & & false & $100 \%$ \\
\hline Strong & $\mathrm{x}$ & & possible & - \\
\hline Uncopyable & $\mathrm{x}$ & $\mathrm{x}$ & true & $100 \%$ \\
\hline Visible & & $\mathrm{x}$ & possible & - \\
\hline Weak & & $\mathrm{x}$ & possible & - \\
\hline
\end{tabular}

Figure A.2: Central processing system properties as identified by the two participants \#1 and \#2. The last columns shows the percentage of participants that gave the correct answer, except for those properties that are ambiguous. 


\begin{tabular}{|c|c|c|c|c|c|c|c|c|c|c|}
\hline Property & $\# 1$ & $\# 2$ & $\# 3$ & \#4 & $\# 5$ & \#6 & \#7 & \#8 & $\begin{array}{l}\text { Model } \\
\text { Answer }\end{array}$ & $\begin{array}{l}\text { Correctnes } \\
\%\end{array}$ \\
\hline Active & $\mathrm{x}$ & & & & & & & $\mathrm{x}$ & false & 75 \\
\hline Cohesive & $\mathrm{x}$ & $\mathrm{x}$ & $\mathrm{x}$ & & & $\mathrm{x}$ & $\mathrm{x}$ & & true & 63 \\
\hline Decaying & & & & & $\mathrm{x}$ & & & & false & 88 \\
\hline Dense & & $\mathrm{x}$ & & & $\mathrm{x}$ & & & $\mathrm{x}$ & true & 38 \\
\hline $\begin{array}{l}\text { Difficultly } \\
\text { manufacturable }\end{array}$ & $\mathrm{x}$ & $\mathrm{x}$ & $\mathrm{x}$ & $\mathrm{x}$ & $\mathrm{x}$ & $\mathrm{x}$ & & $\mathrm{x}$ & true & 88 \\
\hline $\begin{array}{l}\text { Easily } \\
\text { manufacturable }\end{array}$ & & & & & & $\mathrm{x}$ & & & false & 88 \\
\hline $\begin{array}{l}\text { Enclosed with } \\
\text { opening }\end{array}$ & & & & & & $\mathrm{x}$ & & & false & 88 \\
\hline Identifiable & $\mathrm{x}$ & $\mathrm{x}$ & & $\mathrm{x}$ & & & $\mathrm{x}$ & $\mathrm{x}$ & true & 63 \\
\hline Inert & & $\mathrm{x}$ & $\mathrm{x}$ & $\mathrm{x}$ & $\mathrm{x}$ & $\mathrm{x}$ & $\mathrm{x}$ & $\mathrm{x}$ & true & 88 \\
\hline Invisible & & & & & & & & & false & 100 \\
\hline Large & $\mathrm{x}$ & $\mathrm{x}$ & $\mathrm{x}$ & $\mathrm{x}$ & $\mathrm{x}$ & $\mathrm{x}$ & & $\mathrm{x}$ & true & 88 \\
\hline Limited spacious & & & $\mathrm{x}$ & & & $\mathrm{x}$ & & & false & 75 \\
\hline Modifiable & $\mathrm{x}$ & & & & & $\mathrm{x}$ & $\mathrm{x}$ & & false & 63 \\
\hline Movable & & & & & & & $\mathrm{x}$ & & false & 88 \\
\hline Slow & & & & & & & $\mathrm{x}$ & & false & 88 \\
\hline Sound producing & & $\mathrm{x}$ & & $\mathrm{x}$ & $\mathrm{x}$ & & $\mathrm{x}$ & $\mathrm{x}$ & true & 63 \\
\hline Strong & $\mathrm{x}$ & $\mathrm{x}$ & $\mathrm{x}$ & $\mathrm{x}$ & $\mathrm{x}$ & $\mathrm{x}$ & & $\mathrm{x}$ & true & 88 \\
\hline Uncopyable & $\mathrm{x}$ & & & & & $\mathrm{x}$ & & & possible & - \\
\hline Visible & $\mathrm{x}$ & $\mathrm{x}$ & $\mathrm{x}$ & $\mathrm{x}$ & $\mathrm{x}$ & $\mathrm{x}$ & $\mathrm{x}$ & $\mathrm{x}$ & true & 100 \\
\hline Weak & & & & & & & & & false & 100 \\
\hline
\end{tabular}

Figure A.3: Card reader properties as identified by the participants. 


\begin{tabular}{|c|c|c|c|c|c|c|c|c|c|c|c|}
\hline Property & $\# 1$ & $\# 2$ & $\# 3$ & $\# 4$ & \#5 & $\# 6$ & $\# 7$ & \#8 & \#9 & $\begin{array}{l}\text { Model } \\
\text { Answer }\end{array}$ & $\begin{array}{l}\text { Correctness } \\
\%\end{array}$ \\
\hline Active & $\mathrm{x}$ & & $\mathrm{x}$ & $\mathrm{x}$ & $\mathrm{x}$ & $\mathrm{x}$ & $\mathrm{x}$ & $\mathrm{x}$ & & false & 22 \\
\hline Cohesive & & $\mathrm{x}$ & & & & $\mathrm{x}$ & & & $\mathrm{x}$ & true & 33 \\
\hline Decaying & $\mathrm{x}$ & $\mathrm{x}$ & $\mathrm{x}$ & $\mathrm{x}$ & $\mathrm{x}$ & $\mathrm{x}$ & & $\mathrm{x}$ & & true & 78 \\
\hline Dense & & $\mathrm{x}$ & & & $\mathrm{x}$ & $\mathrm{x}$ & & $\mathrm{x}$ & $\mathrm{x}$ & true & 56 \\
\hline $\begin{array}{l}\text { Difficultly } \\
\text { manufacturable }\end{array}$ & $\mathrm{x}$ & $\mathrm{x}$ & & $\mathrm{x}$ & & $\mathrm{x}$ & $\mathrm{x}$ & & $\mathrm{x}$ & true & 67 \\
\hline $\begin{array}{l}\text { Easily } \\
\text { manufacturable }\end{array}$ & & & $\mathrm{x}$ & & & & & $\mathrm{x}$ & & false & 78 \\
\hline $\begin{array}{l}\text { Enclosed with } \\
\text { opening }\end{array}$ & & & & & $\mathrm{x}$ & & & & & false & 89 \\
\hline Identifiable & $\mathrm{x}$ & $\mathrm{x}$ & $\mathrm{x}$ & $\mathrm{x}$ & & $\mathrm{x}$ & $\mathrm{x}$ & $\mathrm{x}$ & $\mathrm{x}$ & true & 89 \\
\hline Inert & & & & & & & & & & true & 0 \\
\hline Invisible & & & & & & & & & & false & 100 \\
\hline Large & & & & & & & & & $\mathrm{x}$ & false & 89 \\
\hline Limited spacious & & & $\mathrm{x}$ & & & & & $\mathrm{x}$ & $\mathrm{x}$ & false & 67 \\
\hline Modifiable & & & $\mathrm{x}$ & $\mathrm{x}$ & $\mathrm{x}$ & $\mathrm{x}$ & $\mathrm{x}$ & $\mathrm{x}$ & & false & 33 \\
\hline Movable & & $\mathrm{x}$ & $\mathrm{x}$ & $\mathrm{x}$ & $\mathrm{x}$ & $\mathrm{x}$ & $\mathrm{x}$ & $\mathrm{x}$ & $\mathrm{x}$ & true & 89 \\
\hline Slow & & $\mathrm{x}$ & $\mathrm{x}$ & & & & $\mathrm{x}$ & $\mathrm{x}$ & $\mathrm{x}$ & false & 44 \\
\hline Sound producing & $\mathrm{x}$ & & & & & & & & & false & 89 \\
\hline Strong & & & & & & & & & & false & 100 \\
\hline Uncopyable & $\mathrm{x}$ & & & $\mathrm{x}$ & & & & & & true & 22 \\
\hline Visible & $\mathrm{x}$ & $\mathrm{x}$ & $\mathrm{X}$ & $\mathrm{x}$ & $\mathrm{x}$ & & $\mathrm{x}$ & $\mathrm{x}$ & $\mathrm{x}$ & true & 89 \\
\hline Weak & & $\mathrm{x}$ & $\mathrm{x}$ & $\mathrm{x}$ & $\mathrm{x}$ & $\mathrm{x}$ & & & $\mathrm{x}$ & true & 67 \\
\hline
\end{tabular}

Figure A.4: Chip card properties as identified by the participants. 


\begin{tabular}{lllll}
\hline Property & $\# 1$ & $\# 2$ & Model Answer & Correctness \% \\
\hline Active & & 1 & false & 50 \\
Cohesive & & 1 & true & 50 \\
Decayling & 1 & & false & 50 \\
Dense & & 1 & true & 50 \\
Difficultly manufacturable & 1 & 1 & true & 100 \\
Easily manufacturable & & & false & 100 \\
Enclosed with opening & & & false & 100 \\
Identifiable & 1 & 1 & true & 100 \\
Inert & & & true & 0 \\
Invisible & $?$ & & false & 100 \\
Large & & & false & 100 \\
Limited spacious & & & false & 100 \\
Modifiable & 1 & & false & 50 \\
Movable & 1 & 1 & true & 100 \\
Slow & & 1 & false & 50 \\
Sound producing & & & false & 100 \\
Strong & & & false & 100 \\
Uncopyable & $?$ & 1 & true & 50 \\
Visible & & 1 & true & 50 \\
Weak & & 1 & true & 50 \\
\hline
\end{tabular}

Figure A.5: Chip properties as identified by the participants. 


\begin{tabular}{llll}
\hline Property & $\# 1$ & Model Answer & Correctness \% \\
\hline Active & & true & 0 \\
Cohesive & & true & 0 \\
Decayling & $\mathrm{x}$ & true & 100 \\
Dense & $\mathrm{X}$ & false & 0 \\
Difficultly manufacturable & & true & 0 \\
Easily manufacturable & & false & 100 \\
Enclosed with opening & & true & 0 \\
Identifiable & $\mathrm{x}$ & true & 100 \\
Inert & & false & 100 \\
Invisible & & false & 100 \\
Large & & true & 0 \\
Limited spacious & & true & 0 \\
Modifiable & & true & 0 \\
Movable & & true & 0 \\
Slow & & false & 100 \\
Sound producing & $\mathrm{x}$ & true & 100 \\
Strong & $\mathrm{x}$ & true & 100 \\
Uncopyable & & false & 100 \\
Visible & & true & 0 \\
Weak & & false & 100 \\
\hline
\end{tabular}

Figure A.6: Bus, tram or metro properties as identified by the participants. 


\section{A.4 Integrated Security Patterns}

\begin{tabular}{|c|c|c|}
\hline 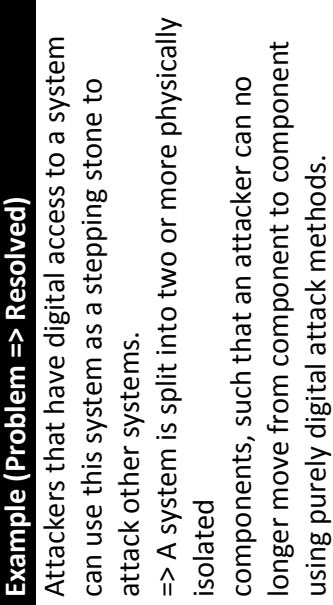 & 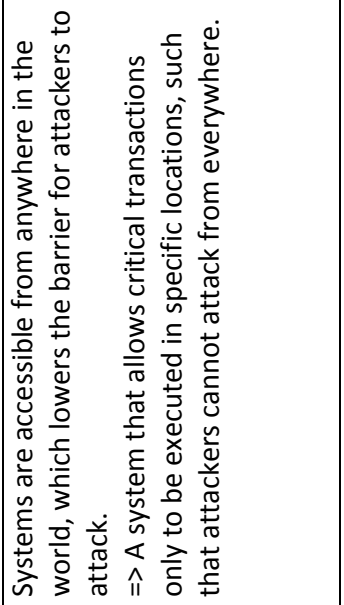 & 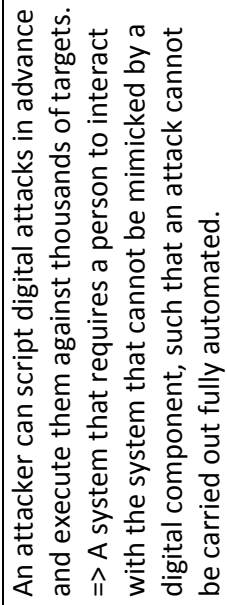 \\
\hline 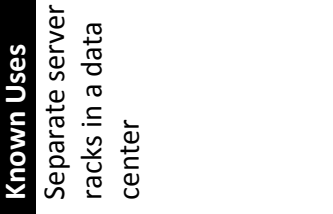 & 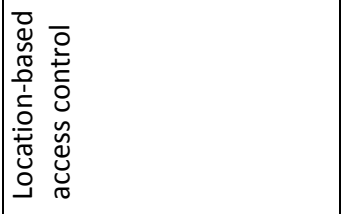 & $\mid \begin{array}{l}0 \\
\frac{\pi}{0} \\
\frac{2}{2} \\
0 \\
0\end{array}$ \\
\hline 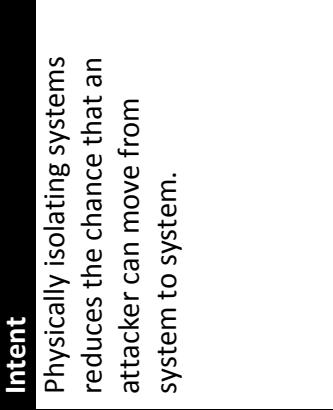 & 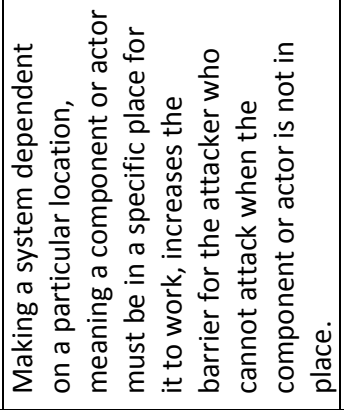 & 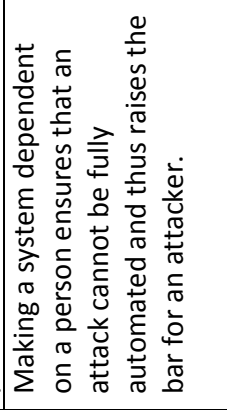 \\
\hline 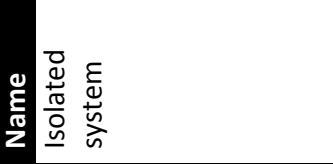 & 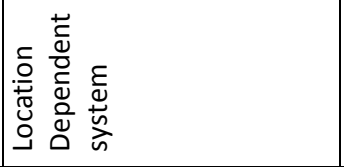 & 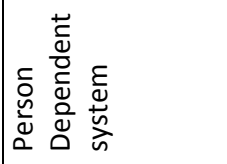 \\
\hline$\#-$ & $\sim$ & \\
\hline
\end{tabular}




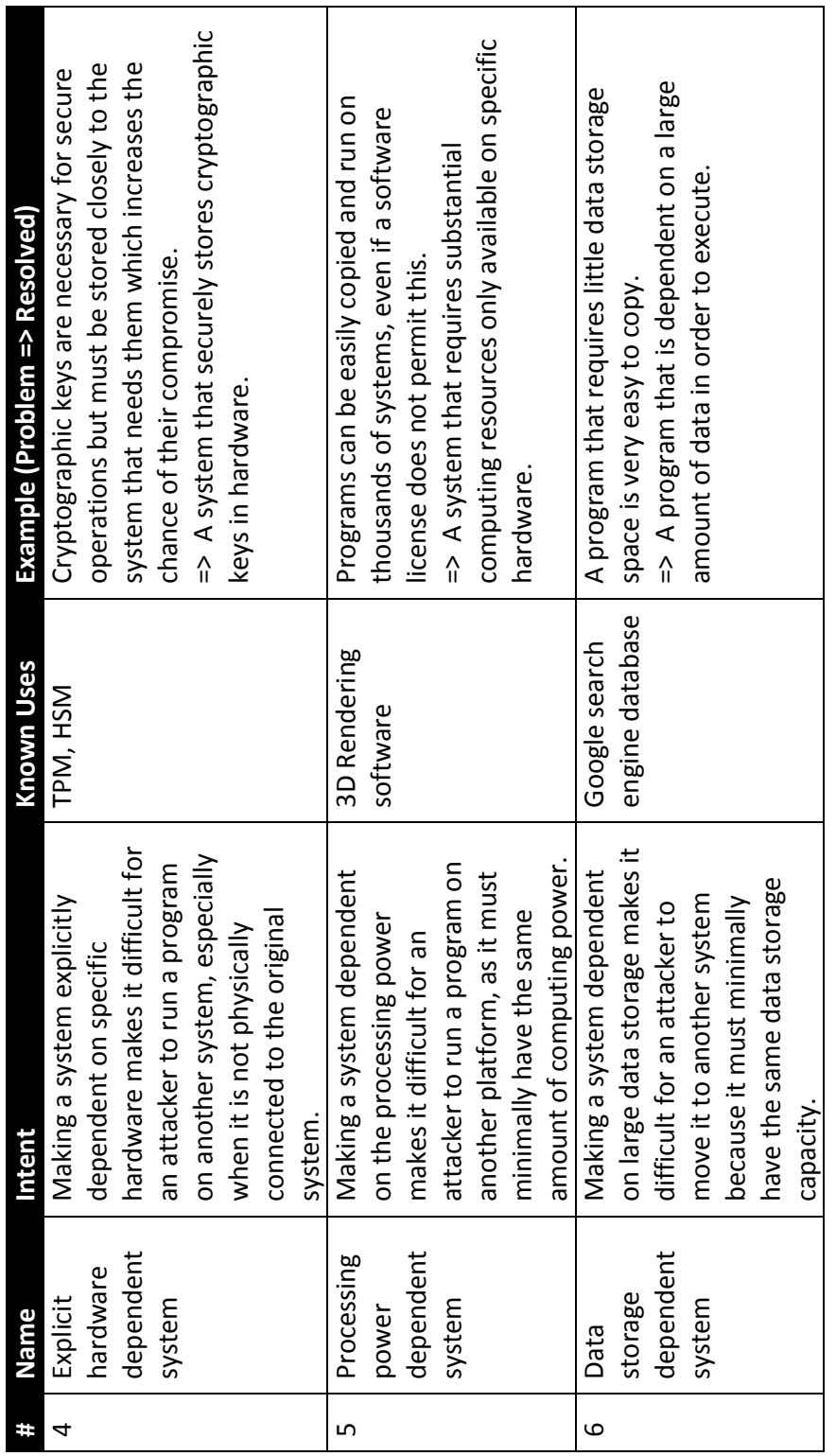




\begin{tabular}{|c|c|c|}
\hline 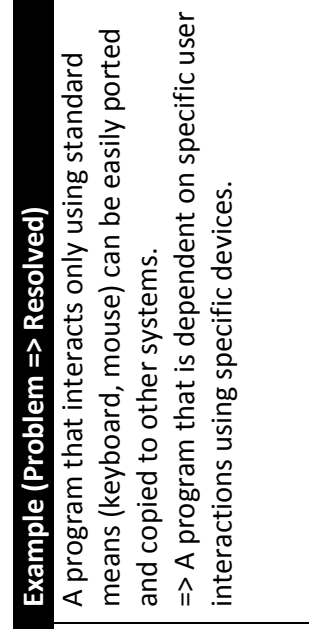 & 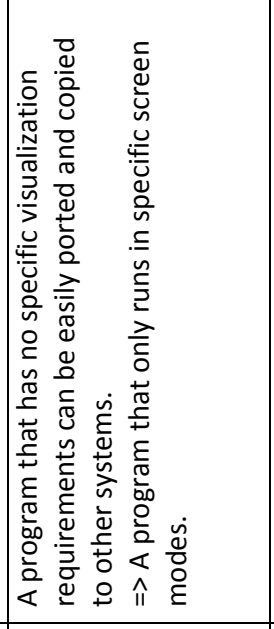 & 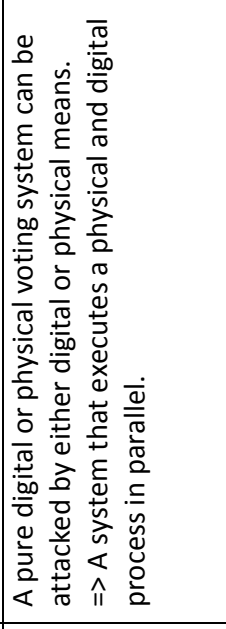 \\
\hline 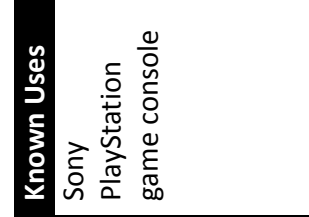 & 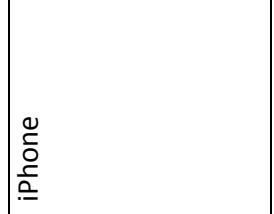 & 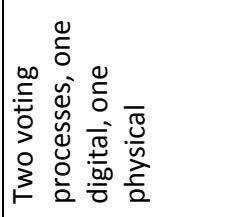 \\
\hline 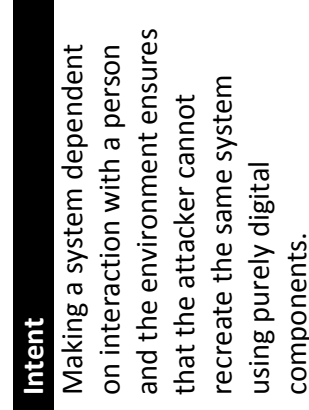 & 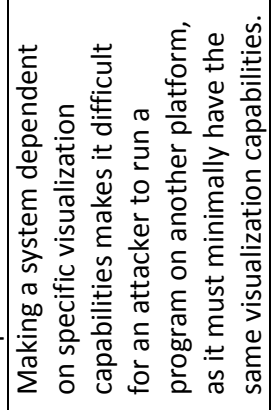 & 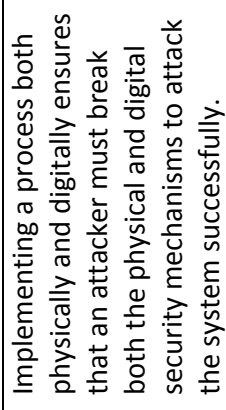 \\
\hline 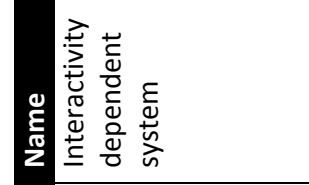 & 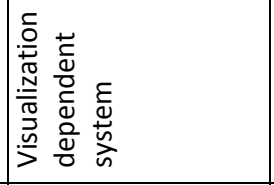 & 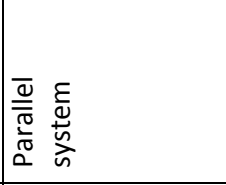 \\
\hline$\# \wedge$ & $\infty$ & $\sigma$ \\
\hline
\end{tabular}




\begin{tabular}{|c|c|c|c|}
\hline 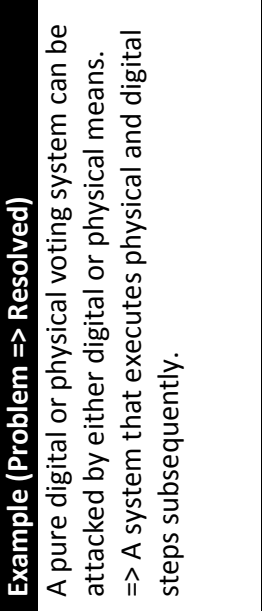 & 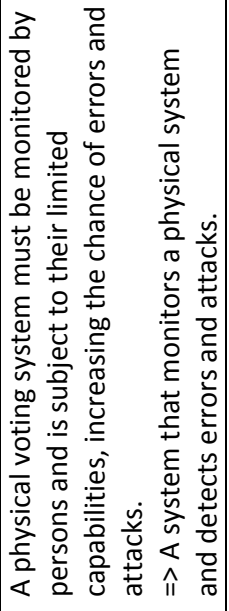 & 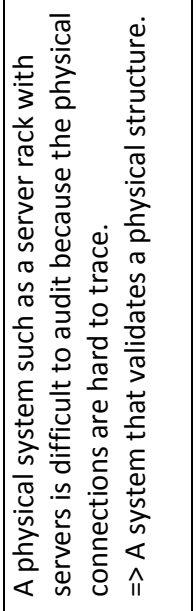 & 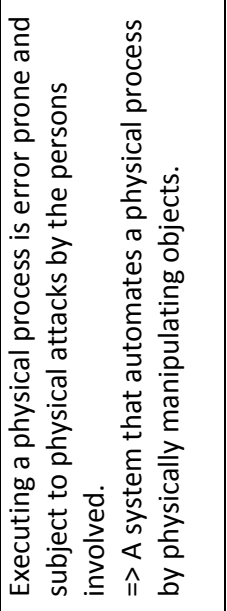 \\
\hline 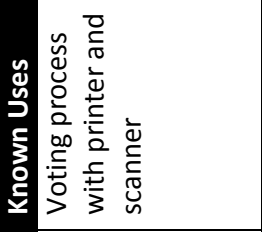 & 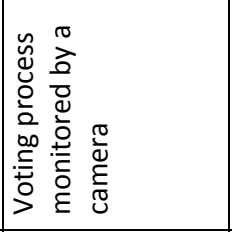 & 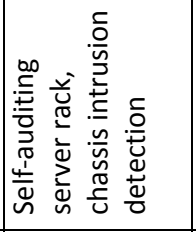 & 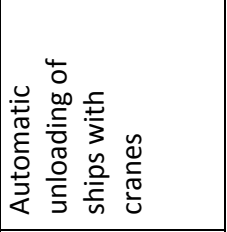 \\
\hline 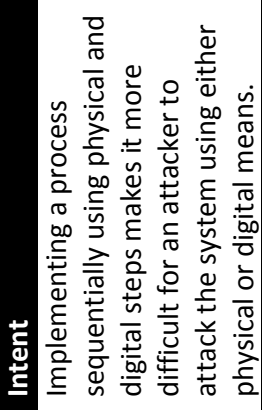 & 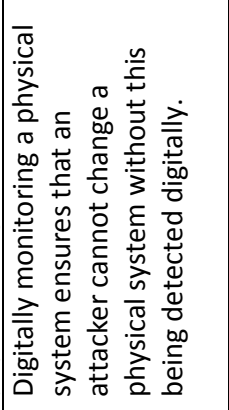 & 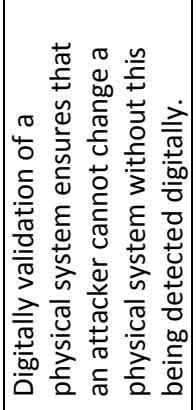 & 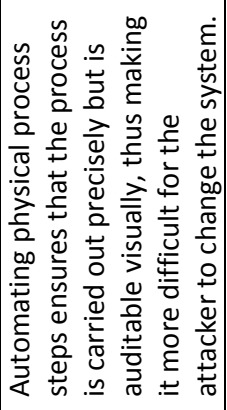 \\
\hline 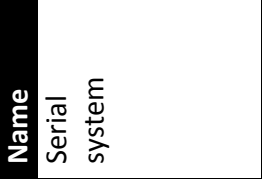 & 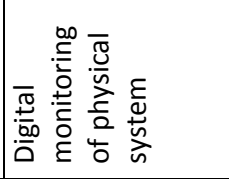 & 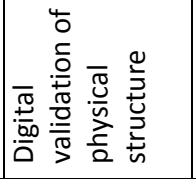 & 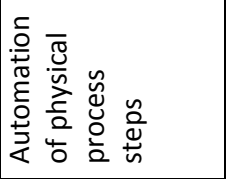 \\
\hline * 우 & $\exists$ & $\approx$ & $\stackrel{m}{r}$ \\
\hline
\end{tabular}




\section{Bibliography}

[1] R. Agrawal, J. Kiernan, R. Srikant, and Y. Xu. Hippocratic databases. Proceedings of the 28th international conference on Very Large Data Bases, 28:143-154, 2002. 26, 27

[2] J.E. Aken. Management research based on the paradigm of the design sciences: The quest for field-tested and grounded technological rules. Journal of management studies, 41(2):219-246, 2004. 50

[3] Amazon Web Services. Amazon Web Services: Overview of Security Processes. http://awsmedia.s3.amazonaws.com/pdf/AWS_ Security_Whitepaper.pdf, 2010. Retrieved 2010-11-06. 148

[4] M. Anand, E. Cronin, M. Sherr, M. Blaze, Z. Ives, and I. Lee. Security challenges in next generation cyber physical systems. Beyond SCADA: Networked Embedded Control for Cyber Physical Systems, 2006. 41

[5] R.J. Anderson. Security Engineering: A Guide to Building Dependable Distributed Systems. Wiley Publishing, 2 edition, 2008. 78, 193

[6] Network World (Andreas M. Antonopoulos). It security's scariest acronym: Byod, bring your own device, 2011. http://www . networkworld. com/columnists/2011/072711-andreas.html,"'Retrieved 2011-07-28”. 32

[7] Apple. Apple's App Store Downloads Top 15 Billion. https: //www.apple.com/pr/library/2011/07/07Apples-AppStore-Downloads-Top-15-Billion.html, 2011. Retrieved 2012-04-23. 186

[8] C. Ardagna, M. Cremonini, E. Damiani, S.D.C. di Vimercati, and P. Samarati. Supporting location-based conditions in access control policies. In 
Proceedings of the 2006 ACM Symposium on Information, computer and communications security, page 222. ACM, 2006. 77, 96

[9] A. Avizienis, J.-C. Laprie, B. Randell, and C. Landwehr. Basic concepts and taxonomy of dependable and secure computing. Dependable and Secure Computing, IEEE Transactions on, 1(1):11-33, 2004. 16

[10] S. Bandhakavi, C. C. Zhang, and M. Winslett. Super-sticky and declassifiable release policies for flexible information dissemination control. In WPES '06: Proceedings of the 5th ACM workshop on Privacy in electronic society, pages 51-58, New York, NY, USA, 2006. ACM. 27

[11] J. Bannet, D.W. Price, A. Rudys, J. Singer, and D.S. Wallach. Hack-a-vote: Security issues with electronic voting systems. Security \& Privacy, IEEE, 2(1):32-37, 2004. 131

[12] B. Barak, O. Goldreich, R. Impagliazzo, S. Rudich, A. Sahai, S. Vadhan, and K. Yang. On the (im)possibility of obfuscating programs. In Joe Kilian, editor, Advances in Cryptology CRYPTO 2001, volume 2139 of Lecture Notes in Computer Science, pages 1-18. Springer Berlin / Heidelberg, 2001. 200

[13] K. Barr, P. Bungale, S. Deasy, V. Gyuris, P. Hung, C. Newell, H. Tuch, and B. Zoppis. The vmware mobile virtualization platform: is that a hypervisor in your pocket? ACM SIGOPS Operating Systems Review, 44(4):124-135, 2010. 32

[14] BBC. Siprnet: Where the leaked cables came from, 2010. http: / / www.bbc.co.uk/news/world-us-canada11863618,"'Retrieved 2011-07-27'. 20

[15] W. Benjamin. The Work of Art in the Age of Mechanical Reproduction. Illuminations: Essays and Reflections, pages 217-52, 1936. 188

[16] S. Berger, R. Caceres, K. Goldman, D. Pendarakis, R. Perez, JR Rao, E. Rom, R. Sailer, W. Schildhauer, D. Srinivasan, et al. Security for the cloud infrastructure: Trusted virtual data center implementation. IBM Journal of Research and Development, 53(4):6, 2010. 148

[17] E. Bertino, P.A. Bonatti, and E. Ferrari. TRBAC: a temporal role-based access control model. In Proceedings of the fifth ACM workshop on Rolebased access control, pages 21-30. ACM, 2000. 101

[18] R. Bhatti, E. Bertino, and A. Ghafoor. A trust-based context-aware access control model for web-services. Distributed and Parallel Databases, 18(1):83-105, 2005. 97 
[19] R. Bhatti, B. Shafiq, M. Shehab, and A. Ghafoor. Distributed access management in multimedia IDCs. Computer, 38(9):60-69, 2005. 96

[20] A. Blaich and A. Striegel. Is High Definition a natural DRM? Computer Communications, pages 1-4, 2009. 209

[21] B. Blakley. The Emperor's old armor. In Proceedings of the 1996 workshop on New security paradigms, pages 2-16. ACM, 1996. 28, 38, 280

[22] S. Bratus, M.E. Locasto, A. Ramaswamy, and S.W. Smith. Traps, events, emulation, and enforcement: managing the yin and yang of virtualizationbased security. In VMSec '08: Proceedings of the 1st ACM workshop on Virtual machine security, pages 49-58, New York, NY, USA, 2008. ACM. $148,165,183$

[23] V. Bryl, F. Dalpiaz, R. Ferrario, and A. Mattioli. Evaluating procedural alternatives: a case study in e-voting. Electronic Government, an International Journal, 6(2):213-231, 2009. 41, 111

[24] J. A. Calandrino, W. Clarkson, and E. W. Felten. Some consequences of paper fingerprinting for elections. In Proceedings of the 2009 conference on Electronic voting technology/workshop on trustworthy elections, EVT/WOTE'09, Berkeley, CA, USA, 2009. USENIX Association. 143

[25] California Institute of Technology and The Massachusetts Institute of Technology Corporation. VOTING: What Is; What Could Be, 2001. 121

[26] A.A. Cárdenas, S. Amin, and S. Sastry. Secure control: Towards survivable cyber-physical systems. In Distributed Computing Systems Workshops, 2008. ICDCS'08. 28th International Conference on, pages 495-500. IEEE, 2008. 41

[27] J.G. Cederquist, R. Corin, M.A.C. Dekker, S. Etalle, J.I. den Hartog, and G. Lenzini. Audit-based compliance control. International Journal of Information Security, 6(2):133-151, 2007. 81

[28] S. Chandran and JBD Joshi. LoT-RBAC: A location and time-based RBAC model. Web Information Systems Engineering-WISE 2005, pages 361-375, 2005. 97

[29] D. Chaum, R. Carback, J. Clark, A. Essex, S. Popoveniuc, R.L. Rivest, P.Y.A. Ryan, E. Shen, and A.T. Sherman. Scantegrity II: End-to-end verifiability for optical scan election systems using invisible ink confirmation codes. In Proceedings of the USENIX/Accurate Electronic Voting Technology Workshop, 2008. 114, 121, 138 
[30] S. Chen, Y. Zhang, and W. Trappe. Inverting sensor networks and actuating the environment for spatio-temporal access control. In Proceedings of the fourth ACM workshop on Security of ad hoc and sensor networks, page 12. ACM, 2006. 97

[31] L. Cheung and C. Newport. Provably secure ciphertext policy ABE. In Proceedings of the 14th ACM conference on Computer and communications security, pages 456-465. ACM, 2007. 27

[32] Citrix. Xenserver administrator's guide. support.citrix. com/servlet/KbServlet/download/18051-102-19048/ reference.pdf, September 2008. Retrieved 2009-03-18. 168

[33] A. van Cleeff, W. Pieters, and R. Wieringa. Benefits of location-based access control: A literature study. In Proceedings of the 2010 IEEE/ACM Int'l Conference on Green Computing and Communications \& Int'l Conference on Cyber, Physical and Social Computing, GREENCOM-CPSCOM '10, pages 739-746, Washington, DC, USA, 2010. IEEE Computer Society. 40

[34] R.H. Coase. The Nature of the Firm. Economica, 4(16):386-405, 1937. 31

[35] J.M. Corbin and A. Strauss. Grounded theory research: Procedures, canons, and evaluative criteria. Qualitative sociology, 13(1):3-21, 1990. 78

[36] Corsec Security. VMware Inc ESX Server 3.0.2 and VirtualCenter 2.0.2. Www.commoncriteriaportal.org/files/epfiles/ vmware-sec-e.pdf, 2008. Common Criteria Security Target, EAL4+, Retrieved 2009-04-20. 161

[37] PCI Security Standard Council. Payment Card Industry (PCI) Data Security Standard. https://www.pcisecuritystandards.org/ security_standards/download.html?id=pci_dss_v11.pdf, September 2006. 29

[38] Common Criteria. Common criteria for information technology security evaluation.http://www.commoncriteriaportal.org/files/ CCfiles/CCPART1V3.1R1.pdf, September 2006. 22

[39] T.D. Crowe and D.L. Zahm. Crime prevention through environmental design. Butterworth-Heinemann Boston, 2000. 101

[40] M.L. Damiani, E. Bertino, and C. Silvestri. Approach to Supporting Continuity of Usage in Location-Based Access Control. In 12th IEEE International Workshop on Future Trends of Distributed Computing Systems, 2008. FTDCS'08, pages 199-205, 2008. 96 
[41] M.L. Damiani, E. Bertino, and C. Silvestri. Spatial Domains for the Administration of Location-based Access Control Policies. Journal of Network and Systems Management, 16(3):277-302, 2008. 79,97,98

[42] M.L. Damiani, H. Martin, Y. Saygin, M.R. Spada, and C. Ulmer. Spatiotemporal access control: challenges and applications. In Proceedings of the 14th ACM symposium on Access control models and technologies, pages 175-176. ACM, 2009. 72

[43] M.L. Damiani and C. Silvestri. Towards movement-aware access control. In Proceedings of the SIGSPATIAL ACM GIS 2008 International Workshop on Security and Privacy in GIS and LBS, pages 39-45. ACM, 2008. 96,97

[44] S. Davtyan, S. Kentros, A. Kiayias, L. Michel, N. Nicolaou, A. Russell, A. See, N. Shashidhar, and A.A. Shvartsman. Taking total control of voting systems: Firmware manipulations on an optical scan voting terminal. In Proceedings of the 2009 ACM symposium on Applied Computing, pages 2049-2053. ACM, 2009. 114

[45] R.J. Deibert. Dark guests and great firewalls: The internet and chinese security policy. Journal of Social Issues, 58(1):143-159, 2002. 30

[46] F. den Braber, T. Dimitrakos, B.A. Gran, M.S. Lund, H. Stølen, and J.Ø Aagedal. The coras methodology: model-based risk assessment using uml and up. UML and the Unified Process, pages 332-357, 2003. 251

[47] D.E. Denning and P.F. MacDoran. Location-based authentication: Grounding cyberspace for better security. Computer Fraud \& Security, 1996(2):12-16, 1996. 39, 72, 99

[48] T. Dimkov, W. Pieters, and P. Hartel. Portunes: representing attack scenarios spanning through the physical, digital and social domain. In ARSPAWITS, 2010. 41, 78

[49] Distributed Management Task Force, Inc. Open virtualization format specification. Www.dmtf.org/standards/published_documents/ DSP0243_1.0.0.pdf, February 2009. Version 1.0.0, Retrieved 200904-20. 165

[50] C. Doctorow. The Coming War on General Purpose Computation, 2011. http://boingboing.net/2011/12/27/the-comingwar-on-general-purp.html, 283

[51] C. Dougherty, K. Sayre, R. C. Seacord, D. Svoboda, and K. Togashi. Secure design patterns. Technical Report CMU/SEI-2009-TR-010, Carnegie- 
Mellon University Pittsburgh PA Software Engineering Institute, 2009. 9 . 46, 62, 65, 252, 254

[52] G.W. Dunlap, S. T. King, S. Cinar, M.A. Basrai, and P.M. Chen. Revirt: Enabling intrusion analysis through virtual-machine logging and replay. In In Proceedings of the 2002 Symposium on Operating Systems Design and Implementation (OSDI), pages 211-224, New York, NY, USA, 2002. ACM. 148, 166

[53] K.M. Eisenhardt. Building theories from case study research. Academy of management review, pages 532-550, 1989. 46, 56, 68, 220

[54] C. Everett. Is DRM fit for purpose? Computer Fraud \& Security, 2010(4):5 $-7,2010.185$

[55] A.J. Feldman, J.A. Halderman, and E.W. Felten. Security analysis of the diebold accuvote-ts voting machine. In Proceedings of the USENIX Workshop on Accurate Electronic Voting Technology, pages 2-2. USENIX Association, 2007. 114, 127

[56] E. Fernandez, J. Ballesteros, A. Desouza-Doucet, and M. Larrondo-Petrie. Security patterns for physical access control systems. In Steve Barker and Gail-Joon Ahn, editors, Data and Applications Security XXI, volume 4602 of Lecture Notes in Computer Science, pages 259-274. Springer Berlin Heidelberg, 2007. 252

[57] D. Ferraiolo, J. Cugini, and D.R. Kuhn. Role-based access control (rbac): Features and motivations. In Proceedings of 11th Annual Computer Security Application Conference, pages 11-15, 1995. 78, 89

[58] L. Floridi. The ontological interpretation of informational privacy. Ethics and Information Technology, 7(4):185-200, 2005. 37, 280

[59] Jericho Forum. An Overview and How to Get Involved. http://www . opengroup.org/jericho/brochureJF080402.pdf, 2007. 20

[60] Jericho Forum. Jericho Forum Report Back. https: //www.opengroup.org/projects/jericho/Webinar_ Dec09_d4.pdf, 2009.4

[61] V.N.L. Franqueira, A. van Cleeff, P. van Eck, and R. Wieringa. External insider threat: a real security challenge in enterprise value webs. In 2010 International Conference on Availability, Reliability and Security, pages 446-453. IEEE, 2010. 21 
[62] E. Gamma, R. Helm, R. Johnson, and J. Vlissides. Design patterns: Abstraction and reuse of object-oriented design. Springer, 1993. 252

[63] S.L. Garfinkel, A. Juels, and R. Pappu. Rfid privacy: an overview of problems and proposed solutions. Security Privacy, IEEE, 3(3):34 - 43, mayjune 2005. 34

[64] T. Garfinkel, B. Pfaff, J. Chow, M. Rosenblum, and D. Boneh. Terra: a virtual machine-based platform for trusted computing. SIGOPS Oper. Syst. Rev., 37(5):193-206, 2003. 167

[65] T. Garfinkel and M. Rosenblum. A virtual machine introspection based architecture for intrusion detection. In Proceedings of Network and Distributed Systems Security Symposium, pages 191-206, 2003. 166

[66] T. Garfinkel and M. Rosenblum. When virtual is harder than real: Security challenges in virtual machine based computing environments. In 10th Workshop on Hot Topics in Operating Systems, 2005. 167, 168, 169

[67] T. Garfinkel and A. Warfield. What virtualization can do for security. ; $L O$ GIN:, 32(6):28-34, December 2007. 166

[68] G. Gebel. Federated Identity: A Progress Report. In ISSE 2005 Securing Electronic Business Processes: Highlights of the Information Security Solutions Europe 2005 Conference. Springer, 2005. 32

[69] C. Gebhardt and A Tomlinson. Security consideration for virtualization. Technical Report RHUL-MA-2008-16, Royal Holloway, University of London, Egham, Surrey TW20 0EX, England, 2008. 168

[70] C. Gentry. Fully homomorphic encryption using ideal lattices. In Proceedings of the 4lst annual ACM symposium on Theory of computing, pages 169-178. ACM, 2009. 28

[71] S.N. Goggin and M.D. Byrne. An examination of the auditability of voter verified paper audit trail (vvpat) ballots. In Proceedings of the USENIX Workshop on Accurate Electronic Voting Technology, pages 10-10, Berkeley, CA, USA, 2007. USENIX Association. 114,139

[72] P. Golle and N. Ducheneaut. Preventing bots from playing online games. Computers in Entertainment (CIE), 3(3):3, 2005. 39

[73] R. Gonggrijp and W.J. Hengeveld. Studying the nedap/groenendaal es3b voting computer: A computer security perspective. In Proceedings of the USENIX Workshop on Accurate Electronic Voting Technology, pages 1-1. USENIX Association, 2007. 114, 134 
[74] Google. Google health. http://www.google.com/intl/en-US/ health/about/, 2008. 31

[75] T. Grandison, M. Bilger, L. O'Connor, M. Graf, M. Swimmer, M. Schunter, A. Wespi, and N. Zunic. Elevating the discussion on security management: The data centric paradigm. In Business-Driven IT Management, 2007. BDIM '07. 2nd IEEE/IFIP International Workshop on, pages 84-93. IEEE, May 2007. 26

[76] R. Gross and A. Acquisti. Information revelation and privacy in online social networks. In Proceedings of the 2005 ACM workshop on Privacy in the electronic society, pages 71-80. ACM, 2005. 17

[77] The Open Group. TOGAF Version 9, 2011. http: //pubs.opengroup.org/architecture/togaf9-doc/ arch/,'Retrieved 2011-07-28". 40

[78] J. Grove. ACM statement on voting systems. Communications of the ACM, 47(10):69-70, 2004. 110

[79] S.K.S. Gupta, T. Mukherjee, K. Venkatasubramanian, and T.B. Taylor. Proximity based access control in smart-emergency departments. In Proceedings - Fourth Annual IEEE International Conference on Pervasive Computing and Communications Workshops, PerCom Workshops 2006, volume 2006, pages 512-516, 2006. 99

[80] C.B. Haley, R. Laney, JD Moffett, and B. Nuseibeh. Security requirements engineering: A framework for representation and analysis. IEEE Transactions on Software Engineering, 34(1):133-153, 2008. 49

[81] J.P. Harris. Election administration in the United States. The Brookings Institution, 1934. 112, 121

[82] S. Harris. CISSP all-in-one exam guide. McGraw-Hill, 4th edition, 2008. 74,78

[83] B. Hayes. Cloud computing. Communications of the ACM, 51(7):9-11, 2008. 22

[84] M.T. Hoesing. Virtualization Security Assessment. Information Security Journal: A Global Perspective, 18(3):124-130, 2009. 39

[85] J. Holler, V. Tsiatsis, C. Mulligan, S. Avesand, S. Karnouskos, and D. Boyle. From Machine-to-machine to the Internet of Things: Introduction to a New Age of Intelligence. Academic Press, 2014. 283 
[86] V. Hourdin, J.Y. Tigli, S. Lavirotte, G. Rey, and M. Riveill. Contextsensitive authorization in interaction patterns. In Proceedings of the 6th International Conference on Mobile Technology, Application \& Systems, pages 1-8. ACM, 2009. 100

[87] V.C. Hu, D.F. Ferraiolo, and D.R. Kuhn. Assessment of access control systems. Interagency Report, 7316:20899-8930, 2006. 79

[88] R.J. Hulsebosch, A.H. Salden, M.S. Bargh, P.W.G. Ebben, and J. Reitsma. Context sensitive access control. In Proceedings of the tenth ACM symposium on Access control models and technologies, pages 111-119. ACM New York, NY, USA, 2005. 72, 77, 79, 97, 100

[89] IEEE P1817 Working Group. Initial Technical Description Of The P1817 Standard. http://grouper.ieee.org/groups/1817/pub_ docs/TechDescription_095.pdf/Retrieved 2010-08-18. 186

[90] International Organization for Standardization (ISO/IEC). ISO/IEC 74982:1989 Information technology - Open Systems Interconnection - Basic Reference Model: The Basic Model, 1989. 16

[91] International Organization for Standardization (ISO/IEC). ISO/IEC 133351:2004 Information technology - Security techniques - Management of information and communications technology security - Part 1: Concepts and models for information and communications technology security management, 2004. 3, 16, 188

[92] International Organization for Standardization (ISO/IEC). ISO/IEC 27001:2005 Information technology - Security techniques - Code of Practice for Information Security Management, 2005. 16, 23

[93] International Organization for Standardization (ISO/IEC). ISO/IEC 27002:2005 Information technology - Security techniques - Code of Practice for Information Security Management, 2005. 23

[94] International Organization for Standardization (ISO/IEC). ISO/IEC 27000:2009 Information technology - Security techniques - Overview and vocabulary, 2009. 16, 23, 71,

[95] International Organization for Standardization (ISO/IEC). Preliminary draft for ISO/IEC 27036-3 - Information technology - Security techniques - Information security for supplier relationships - Part 3: Guidelines for ICT supply chain security. http://www.iso.org/iso/iso_catalogue/catalogue_ tc/catalogue_detail.htm?csnumber=59688, 2011.23 
[96] IT Governance Institute. CObIT 4.1. Isaca, Rolling Meadows, IL 60008 USA, 2007. 147, 154

[97] IT Governance Institute. Aligning CobiT 4.1, ITIL V3 and ISO/IEC 27002 for Business Benefit. ITGI, Rolling Meadows, IL 60008 USA, 2008. 154

[98] B. Jacobs. Architecture is politics: Security and privacy issues in transport and beyond. In Data Protection in a Profiled World, pages 289-299. Springer, 2010. 263

[99] B. Jacobs and W. Pieters. Electronic voting in the netherlands: from early adoption to early abolishment. Foundations of Security Analysis and Design $V$, pages 121-144, 2009. 114

[100] B. Jacobs, W. Pieters, and M. Warnier. Statically checking confidentiality via dynamic labels. In Proceedings of the 2005 workshop on Issues in the theory of security, pages 50-56. ACM, 2005. 18

[101] B. Jansen, H.G.V. Ramasamy, M. Schunter, and A. Tanner. Architecting Dependable and Secure Systems Using Virtualization. Lecture Notes In Computer Science, pages 124-149, 2008. 161, 170, 176

[102] W. Jansen, S. Gavrila, and V. Korolev. Proximity-based authentication for mobile devices. In Proceedings of The 2005 International Conference on Security and Management, SAM'05, pages 398-404, 2005. 96

[103] The Open Group Jericho Forum. Jericho whitepaper. http://www . opengroup.org/jericho/vision_wp.pdf, 2005. 20, 21

[104] The Open Group Jericho Forum. Jericho forum commandments. http: //www.opengroup.org/jericho/commandments_v1.2.pdf. 2007. 4, 25, 26

[105] The Open Group Jericho Forum. White paper business rationale for deperimeterisation. http://opengroup.org/jericho/Business_ Case_for_DP_v1.0.pdf, 2007.20

[106] The Open Group Jericho Forum. Jericho forum - about us. http: //www . opengroup.org/jericho/about.htm, 2008. 20

[107] The Open Group Jericho Forum. Position Paper - COA Framework. http://www.opengroup.org/jericho/COAFwk_v2.0. pdf, 2008. 4, 25

[108] The Open Group Jericho Forum. Cloud Cube Model. http://www . opengroup.org/jericho/cloud_cube_model_v1.0.pdf, 2009. 4 
[109] The Open Group Jericho Forum. Secure Business Collaboration: Do It Now! https://opengroup.org/jericho/COAandCloud_ SCWC.pdf, 2009. 26, 29

[110] R. Johnson, R. Helm, J. Vlissides, and E. Gamma. Design Patterns: Elements of Reusable Object-Oriented Software. Addison-Wesley Professional, 1995. 65

[111] D.W. Jones. Chain voting. In Developing an Analysis of Threats to Voting Systems: Preliminary Workshop Summary, pages 53-55, 2005. 122

[112] D.W. Jones. Counting ballots, 2005. http://www.divms.uiowa. edu/ jones/voting/counting.html, Retrieved 2011-11-03.1123

[113] D.W. Jones. Threats to voting systems. In Developing an Analysis of Threats to Voting Systems: Preliminary Workshop Summary, pages 171179, 2005. 121

[114] H.L. Jonker. Security Matters: Privacy in Voting and Fairness in Digital Exchange. $\mathrm{PhD}$ thesis, University of Luxembourg/Technische Universiteit Eindhoven, 2009. 115

[115] J.B.D. Joshi, R. Bhatti, E. Bertino, and A. Ghafoor. Access-control language for multidomain environments. IEEE Internet Computing, pages 40-50, 2004. 96

[116] C. Kalloniatis, E. Kavakli, and S. Gritzalis. Addressing privacy requirements in system design: the PriS method. Requirements Engineering, 13(3):241-255, 2008. 115

[117] P.A. Karger and D.R. Safford. I/O for Virtual Machine Monitors: Security and Performance Issues. IEEE Security \& Privacy, 6(5):16-23, 2008. 165 . 166,167

[118] G. Kim. Seven steps to a secure virtual environment. Network Security, 2008(8):14-18, 2008. 39

[119] Y.G. Kim, C.J. Mon, D. Jeong, J.O. Lee, C.Y. Song, and D.K. Baik. Context-aware access control mechanism for ubiquitous applications. Advances in Web Intelligence, pages 236-242, 2005. 97

[120] M. Kirkpatrick and E. Bertino. Context-Dependent Authentication and Access Control. iNetSec 2009-Open Research Problems in Network Security, pages $63-75,2009$. 98 
[121] Wired (David Kravets). Internet 'kill switch' legislation back in play, 2011. http://www.wired.com/threatlevel/2011/01/ kill-switch-legislation/,"Retrieved 2011-07-27". 30

[122] R. Krimmer and M. Volkamer. Bits or paper? comparing remote electronic voting to postal voting. In EGOV (Workshops and Posters), pages 225-232, 2005. 115

[123] B.H. Krogh, E. Lee, I. Lee, A. Mok, R. Rajkumar, L.R. Sha, A.S. Vincentelli, K. Shin, J. Stankovic, J. Sztipanovits, et al. Cyber-Physical Systems, Executive Summary, 2008. 41

[124] W. Ku and C.H. Chi. Survey on the technological aspects of digital rights management. Information Security, pages 391-403, 2004. 186, 187

[125] M. Kumar and R.E. Newman. STRBAC - An approach towards spatiotemporal role-based access control. In Proceedings of the Third IASTED International Conference on Communication, Network, and Information Security, CNIS 2006, pages 150-155, 2006. 96,97

[126] G. Lawton. New technology prevents data leakage. Computer, 41(9):1417, 2008. 192

[127] B. Laxton, K. Wang, and S. Savage. Reconsidering physical key secrecy: Teleduplication via optical decoding. In Proceedings of the 15th ACM conference on Computer and communications security, pages 469-478. ACM, 2008. 84

[128] A. Lazouski, F. Martinelli, and P. Mori. Usage control in computer security: A survey. Computer Science Review, 4(2):81-99, 2010. 68,74

[129] L. Lessig. Code: Version 2.0. Basic Books, Inc., New York, NY, USA, 2006. 30, 38, 189, 193, 196, 199

[130] The British Informatics Society Limited. Block criminal clouds. ISNOW, 5(Part 2):12-13, Winter 2010. http: / /www . infosec.co.uk/ ExhibitorLibrary/251/ISNOW-Winter2010_23.pdf, 30

[131] A. Marinos and G. Briscoe. Community cloud computing. Cloud Computing, pages $472-484,2009.32$

[132] S. Mauw and M. Oostdijk. Foundations of attack trees. Information Security and Cryptology-ICISC 2005, pages 186-198, 2006. 51

[133] H. Meer. On screen keyboards considered harmful today, 2011. http:// thinkst.com/stuff/ocv/osk-thinkst.pdf, Retrieved 201108-02. 34 
[134] Microsoft. Microsoft healthvault. http://www.healthvault. $\mathrm{com} /$, 2008. 31

[135] Microsoft. Microsoft passport. http://www.passport.net, 2008. 30

[136] Microsoft Corporation. Windows Server 2008 Hyper-V Technical Overview. http://www.microsoft.com/ windowsserver2008/en/us/hyperv-overview.aspx, 2008. Retrieved 2009-04-20. 167

[137] Ministery van Binnenlandse Zaken en Koninkrijksrelaties. Werkmap voor stembureauleden - versie 'stemmen in een willekeurig stemlokaal', 2009. (in Dutch). 113,121

[138] David L. Morgan. Focus groups as qualitative research. Sage Publications, Inc, 1988. 9, 46, 65

[139] A. Mowshowitz. Virtual organization. Communications of the ACM, 40(9):30-37, 1997. 32

[140] A. Muhlbauer, R. Safavi-Naini, F. Salim, N.P. Sheppard, and M. Surminen. Location constraints in digital rights management. Computer Communications, 31(6):1173-1180, 2008. 96

[141] G. Myles and S. Nusser. Content protection for games. IBM systems journal, 45(1):119-143, 2006. 193, 199

[142] K. Nance, M. Bishop, and B. Hay. Virtual Machine Introspection: Observation or Interference? IEEE Security \& Privacy, 6(5):32-37, 2008. 166. 167

[143] NEDAP/GROENENDAAL.

Handleiding integraal stem systeem gemeenten, December 2011. http:// wijvertrouwenstemcomputersniet.nl/images/2/22/ Nedap_-_handleiding_ISS_-_gemeenten.pdf. 114

[144] Nederlands Forensisch Instituut. Deskundigenrapport, Zaaknummer (2006.04.03.004), June 2006. http:// wijvertrouwenstemcomputersniet.nl/images/6/60/ NFI_rapport_Te_Meerman.pdf, 114, 129

[145] W.L. Neuman. Social research methods: Qualitative and quantitative approaches, volume 8 . Allyn and Bacon, 2003. 46 
[146] S. Newman and C. Marshall. Pushing toulmin too far: Learning from an argument representation scheme. Xerox PARC, Palo Alto, CA, USA, Technical Report SSL-92-45, 1991. 49, 50

[147] J. Nielsen. Usability engineering. Elsevier, 1994. 262, 263

[148] NIST. CVE-2009-1244. http://web.nvd.nist.gov/view/ vuln/detail?vulnId=CVE-2009-1244, April 2009. Retrieved 2009-04-20. 166

[149] L.D. Norden. The Machinery of Democracy: Voting System Security, Accessibility, Usability, and Cost. Brennan Center for Justice at NYU School of Law, 2006. 121

[150] NSF. Report: Cyber-Physical Systems Summit. http://varma.ece. cmu.edu/Summit/CPS_Summit_Report.pdf, 2008. Retrieved 2011-03-21. 41

[151] J. Oberheide, E. Cooke, and F. Jahanian. Empirical exploitation of live virtual machine migration. In Proceedings of BlackHat DC convention, 2008. 168

[152] A. Odlyzko. Digital rights management: desirable, inevitable, and almost irrelevant. In Proceedings of the 2007 ACM workshop on Digital Rights Management, page 40. ACM, 2007. 193

[153] M. Oh, J. Lee, B.M. Chang, J. Ahn, and K.G. Doh. A programming environment for ubiquitous computing environment. ACM SIGPLAN Notices, 42(4):14-22, 2007. 96, 97

[154] T. Ormandy. An empirical study into the security exposure to host of hostile virtualized environments. In CanSecWest 2007, Vancouver BC, April 2007. 166

[155] OSCE Office for Democratic Institutions and Human Rights (ODIHR). Guidelines for Reviewing a Legal Framework for Elections. ODIHR, 2001. 112,115

[156] OSCE Office for Democratic Institutions and Human Rights (ODIHR). Handbook for Domestic Election Observers. ODIHR, 2003. ISBN 83912750-8-6. 112

[157] OSCE Office for Democratic Institutions and Human Rights (ODIHR). Election Observation Handbook. ODIHR, 5th edition, 2005. ISBN 8360190-00-3. 112, 121, 123 
[158] G. Palmer. De-perimeterisation: Benefits and limitations. Information Security Technical Report, 10(4):189-203, 2005. 26

[159] M. Parameswaran, X. Zhao, A.B. Whinston, and F. Fang. Reengineering the internet for better security. Computer, 40(1):40-44, 2007. 30

[160] H. Pardue, J. Landry, and A. Yasinsac. A Risk Assessment Model for Voting Systems using Threat Trees and Monte Carlo Simulation. In Proceedings of the 2009 First International Workshop on Requirements Engineering for e-Voting Systems, pages 55-60. IEEE Computer Society, 2009. 42,111

[161] J. Park and R. Sandhu. Towards usage control models: beyond traditional access control. In Proceedings of the seventh ACM symposium on Access control models and technologies, pages 57-64. ACM, 2002. 74, 193

[162] S.H. Park, J.S. Cho, Y.J. Han, and T.M. Chung. Design and Implementation of Context-Aware Security Management System for Ubiquitous Computing Environment. In Frontiers of High Performance Computing and Networking ISPA 2007 Workshops, pages 235-244. Springer, 2007. 97

[163] N. Patel. Physical Keygen: Duplicating House Keys on a 3D Printer, 2011. http://eclecti.cc/hardware/physical-keygenduplicating-house-keys-on-a-3d-printer, 84, 283

[164] N. Paul and A.S. Tanenbaum. Trustworthy Voting: From Machine to System. Computer, 42(5):23-29, 2009. 114,131

[165] B.D. Payne, M. Carbone, M. Sharif, and W. Lee. Lares: An architecture for secure active monitoring using virtualization. Security and Privacy, IEEE Symposium on, pages 233-247, 2008. 165

[166] R. Perez, L. van Doorn, and R. Sailer. Virtualization and Hardware-Based Security. IEEE Security \& Privacy, 6(5):24-31, 2008. 39, 161, 165

[167] S. Popoveniuc, J. Clark, R. Carback, A. Essex, and D. Chaum. Securing optical-scan voting. Towards Trustworthy Elections, pages 357-369, 2010. 114

[168] C.W. Probst, R.R. Hansen, and F. Nielson. Where can an insider attack? In Formal Aspects in Security and Trust, volume 4691 of LNCS, pages 127142. Springer Berlin / Heidelberg, 2007. 41, 78, 82

[169] J. Puiggali and V. Morales-Rocha. Remote voting schemes: A comparative analysis. In E-Voting and Identity, volume 4896 of Lecture Notes in Computer Science, pages 16-28. Springer Berlin / Heidelberg, 2007. 115 
[170] I. Ray and M. Kumar. Towards a location-based mandatory access control model. Computers \& Security, 25(1):36-44, 2006. 97

[171] I. Ray, M. Kumar, and L. Yu. LRBAC: A Location-Aware Role-Based Access Control Model. Lecture Notes in Computer Science, 4332:147, 2006. 96

[172] I. Ray and M. Toahchoodee. A spatio-temporal role-based access control model. Lecture Notes in Computer Science (including subseries Lecture Notes in Artificial Intelligence and Lecture Notes in Bioinformatics), 4602 LNCS:211-226, 2007. 97

[173] I. Ray and L. Yu. Short paper: Towards a location-aware role-based access control model. In Proceedings of the First International Conference on Security and Privacy for Emerging Areas in Communications Networks, pages 234-236. IEEE Computer Society, 2005. 96, 97

[174] Respect-IT. KAOS Tutorial v1.0, October 2007. http://www. objectiver.com/fileadmin/download/documents / KaosTutorial.pdf, Retrieved 2009-09-21. 52

[175] E. Rissanen. eXtensible Access Control Markup Language (XACML) Version 3.0 (Committee Specification 01), 2010. http://docs.oasis-open.org/xacml/3.0/xacml-3.0core-spec-cs-01-en.pdf, 75

[176] T. Ristenpart, E. Tromer, H. Shacham, and S. Savage. Hey, you, get off of my cloud: exploring information leakage in third-party compute clouds. In Proceedings of the 16th ACM conference on Computer and communications security, CCS '09, pages 199-212, New York, NY, USA, 2009. ACM. 22

[177] P. Rittwage. C64 Preservation Project - Copy Protection Methods, 2011. http: / / c64preservation.com/dp.php?pg=protection Retrieved 2010-08-18. 200

[178] R.L. Rivest. On the notion of 'software independence' in voting systems. Philosophical Transactions of the Royal Society A: Mathematical, Physical and Engineering Sciences, 366(1881):3759-3767, 2008. 146

[179] Philip Robinson and Jochen Haller. Revisiting the firewall abolition act. Hawaii International Conference on System Sciences, 7:206a, 2003. 23

[180] W. S. Robinson. The logical structure of analytic induction. American Sociological Review, pages 812-818, 1951. 8, 46, 56, 220 
[181] M. Rosenblum and T. Garfinkel. Virtual machine monitors: Current technology and future trends. Computer, 38(5):39-47, 2005. 168

[182] J.H. Saltzer and M.D. Schroeder. The protection of information in computer systems. Proceedings of the IEEE, 63(9):1278-1308, 1975. 81

[183] P. Samuelson. Trade secrets vs. free speech. Communications of the ACM, 46(6):19-23, 2003. 200

[184] R.S. Sandhu, E.J. Coyne, H.L. Feinstein, and C.E. Youman. Role-based access control models. Computer, 29(2):38-47, 1996. 79

[185] R.S. Sandhu and P. Samarati. Access control: principle and practice. Communications Magazine, IEEE, 32(9):40-48, 1994. 73

[186] C. Saran. Windows update blamed for Skype outage. http://www . computerweekly.com/Articles/2007/08/20/226281/ windows-update-blamed-for-skype-outage.htm, Aug 2007. 22

[187] N. Sastry, U. Shankar, and D. Wagner. Secure verification of location claims. In Proceedings of the 2nd ACM workshop on Wireless security, page 10. ACM, 2003. 79,98

[188] A.U. Schmidt, N. Kuntze, and J. Abendroth. Trust for location-based authorisation. In IEEE Wireless Communications and Networking Conference, WCNC, pages 3163-3168, 2008. 97, 282

[189] B. Schneier. Crypto-gram newsletter, May 2001. https://www . schneier.com/crypto-gram-0105.html. 185

[190] B. Schneier. Crypto-gram newsletter, January 2011. http://www . schneier.com/crypto-gram-1101.html. 20

[191] V. Shah and F. Hill. An aspect-oriented security framework. DARPA Information Survivability Conference and Exposition, 2:143, 2003. 41

[192] M.I. Shamos. Paper v. electronic voting records an assessment. In Developing an Analysis of Threats to Voting Systems: Preliminary Workshop Summary, pages 228-254, 2005. 123

[193] Y.C. Shim. Distributed Processing of Context-Aware Authorization in Ubiquitous Computing Environments. Computational Science and Its Applications-ICCSA 2006, pages 125-134, 2006. 97

[194] P. Sieghart. Privacy and computers. Latimer New Dimensions, 1976. as cited in Bennett, Regulating Privacy, p. 17. 37, 280 
[195] T. Sim, S. Zhang, R. Janakiraman, and S. Kumar. Continuous verification using multimodal biometrics. IEEE transactions on pattern analysis and machine intelligence, pages 687-700, 2007. 98

[196] P. Simmonds. De-perimeterisation. http://www.blackhat.com/ presentations/bh-europe-04/bh-eu-04-simmonds.pdf, 2004. Presentation given at BlackHat Europe '04. 20, 26

[197] J.E. Smith and R. Nair. The architecture of virtual machines. Computer, 38(5):32-38, 2005. 149

[198] M. Stieghahn and T. Engel. Law-aware access control for international financial environments. In Proceedings of the Eighth ACM International Workshop on Data Engineering for Wireless and Mobile Access, pages 33 40. ACM, 2009. 96, 97, 100

[199] R. Templeman, Z. Rahman, D. Crandall, and A. Kapadia. Placeraider: Virtual theft in physical spaces with smartphones. arXiv preprint arXiv:1209.5982, 2012. 246

[200] The Register. Webhost hack wipes out data for 100,000 sites. http:// www.theregister.co.uk/2009/06/08/webhost_attack/, 2009. Retrieved 2009-06-25. 170

[201] New York Times. Stuxnet, 2011. http://topics.nytimes.com/ top/reference/timestopics/subjects/c/computer_ malware/stuxnet/index.html,"Retrieved 2011-07-27". 19

[202] New York Times. Wikileaks, 2011. https://www.nytimes. com/interactive/world/statessecrets.html,'Retrieved 2011-07-27”. 19

[203] M. Toahchoodee and I. Ray. On the formal analysis of a spatio-temporal role-based access control model. Lecture Notes in Computer Science (including subseries Lecture Notes in Artificial Intelligence and Lecture Notes in Bioinformatics), 5094 LNCS:17-32, 2008. 81, 97

[204] A. Toninelli, R. Montanari, L. Kagal, and O. Lassila. A semantic contextaware access control framework for secure collaborations in pervasive computing environments. The Semantic Web-ISWC 2006, pages 473-486, 2006. 79, 96, 100

[205] R. Uhlig, G. Neiger, D. Rodgers, AL Santoni, FCM Martins, AV Anderson, SM Bennett, A. Kagi, FH Leung, and L. Smith. Intel virtualization technology. Computer, 38(5):48-56, 2005. 165 
[206] S. Vaarala. Security considerations of commodity x 86 virtualization, May 2006. Helsinki University of Technology, Licentiate Thesis. 161

[207] A. van Cleeff. Realizing security requirements using physical properties: A case study of voting. Technical report, University of Twente, 2010. 109

[208] A van Cleeff, R.J. Pieters, W. Wieringa, and F. van Tiel. Integrated assessment and mitigation of physical and digital security threats: Case studies on virtualization. Information Security Technical Report, -(0):-, 2011. In press. 147, 247

[209] A. van Cleeff, W. Pieters, and R. Wieringa. Benefits of location-based access control: A literature study. In Green Computing and Communications (GreenCom), 2010 IEEE/ACM Int'l Conference on Int'l Conference on Cyber, Physical and Social Computing (CPSCom), pages 739 -746, dec. 2010. 71

[210] A. van Cleeff, W. Pieters, and R.J. Wieringa. Security Implications of Virtualization: A Literature Study. In 2009 International Conference on Computational Science and Engineering, pages 353-358. IEEE, 2009. 147, 148

[211] A. van Cleeff and R.J. Wieringa. Rethinking de-perimeterisation: Problem analysis and solutions. In Proceedings of the IADIS International Conference Information Systems 2009, Barcelona, pages 105-112, Barcelona, February 2009. IADIS press. 19

[212] L. van Doorn. Hardware virtualization trends. In Proceedings of the 2nd international conference on Virtual execution environments, pages 45-45. ACM New York, NY, USA, 2006. 165

[213] J.G. van Eerden and R. de Jong, editors. Fraude en ongewenste beïnloeding bij verkiezingen. Kiesraad, 2008. (in Dutch). 121

[214] A. van Lamsweerde. Requirements Engineering: From System Goals to UML Models to Software Specifications. Wiley, 2009. 51,52

[215] S.J. Vaughan-Nichols. Virtualization Sparks Security Concerns. Computer, 41(8):13-15, 2008. 39, 169

[216] VMware. VMware Infrastructure 3 - Security Hardening. http://www . vmware.com/pdf/vi3_security_hardening_wp.pdf, 2007. Retrieved 2010-10-31. 39, 148

[217] VMware. VMware VMotion datasheet. Www.vmware.com/pdf/ vmotion_datasheet.pdf, 2007. Retrieved 2009-03-18. 168 
[218] VMware. VMware vCenter Update Manager. Www.vmware.com/ files/pdf/update_manager_datasheet.pdf, 2008. Product datasheet, Retrieved 2009-04-20. 168,169

[219] VMware. ESX Server 3 Configuration Guide. http://www . vmware. com/pdf/vi3_35/esx_3/r35/vi3_35_25_3_server_ config.pdf, 2010. Retrieved 2010-11-01. 39, 148

[220] M. Volkamer and R. Vogt. New generation of voting machines in germanythe hamburg way to verify correctness. In Proceedings of the Frontiers in Electronic Elections (FEE 2006) Workshop, 2006. 115

[221] J. Walker. The extended security perimeter. Information Security Technical Report, 10(4):220-227, 2005. 20, 29

[222] A. Wang. The shazam music recognition service. Commun. ACM, 49:4448, August 2006. 187

[223] J. Webster and R. T. Watson. Analyzing the past to prepare for the future: Writing a literature review. MIS Quarterly, 26:13-23, 2002. 8, 53

[224] J. Webster and R.T. Watson. Analyzing the past to prepare for the future: Writing a literature review. MIS Quarterly, 26(2):13-23, 2002. 77

[225] K. Weldemariam and A. Villafiorita. Procedural security analysis: A methodological approach. Journal of Systems and Software, 84(7):1114 - 1129, 2011. 41,111

[226] R. Wieringa. Design Methods for Reactive Systems: Yourdon, Statemate, and the UML. Morgan Kaufmann, 2003. 13, 50

[227] R.J. Wieringa. Design science methodology for information systems and software engineering. Springer, 2014. 65

[228] S. Wolchok, E. Wustrow, J. A. Halderman, H. K. Prasad, A. Kankipati, S. K. Sakhamuri, V. Yagati, and R. Gonggrijp. Security analysis of india's electronic voting machines. In Proceedings of the 17th ACM conference on Computer and communications security, CCS '10, pages 1-14, New York, NY, USA, 2010. ACM. 33, 109

[229] Wired (Mic Wright). Your gas meter: The new frontline in cyberwar, 2011. http://www.wired.co.uk/magazine/ archive/2011/03/start/the-new-front-line-incyberwar?page=2,"Retrieved 2011-07-27'. 20

[230] A. Yasinsac and M. Bishop. The Dynamics of Counting and Recounting Votes. IEEE Security and Privacy, 6(3):22-29, 2008. 109, 117 
[231] H. Zhang, Y. He, and Z. Shi. A formal model for access control with supporting spatial context. Science in China Series F: Information Sciences, 50(3):419-439, 2007. 96

[232] C. Zimmer, B. Bhat, F. Mueller, and S. Mohan. Time-based intrusion detection in cyber-physical systems. In Proceedings of the 1st ACM/IEEE International Conference on Cyber-Physical Systems, pages 109-118. ACM, 2010. 41 


\section{SIKS Dissertation Series}

1998

1998-1 Johan van den Akker (CWI)

DEGAS - An Active, Temporal Database of Autonomous Objects

1998-2 Floris Wiesman (UM)

Information Retrieval by Graphically Browsing Meta-Information

1998-3 Ans Steuten (TUD)

A Contribution to the Linguistic Analysis of Business Conversations within the Language/Action Perspective

1998-4 Dennis Breuker (UM)

Memory versus Search in Games

1998-5 E.W.Oskamp (RUL)

Computerondersteuning bij Straftoemeting

1999

$===$

1999-1 Mark Sloof (VU)

Physiology of Quality Change Modelling; Automated modelling of Quality Change of Agricultural Products

1999-2 Rob Potharst (EUR)

Classification using decision trees and neural nets

1999-3 Don Beal (UM)

The Nature of Minimax Search

1999-4 Jacques Penders (UM)

The practical Art of Moving Physical Objects

1999-5 Aldo de Moor (KUB)

Empowering Communities: A Method for the Legitimate User-Driven Specification of Network Information Systems

1999-6 Niek J.E. Wijngaards (VU)

Re-design of compositional systems

1999-7 David Spelt (UT)

Verification support for object database design 
1999-8 Jacques H.J. Lenting (UM)

Informed Gambling: Conception and Analysis of a Multi-Agent Mechanism for Discrete Reallocation.

2000

$===$

2000-1 Frank Niessink (VU)

Perspectives on Improving Software Maintenance

2000-2 Koen Holtman (TUE)

Prototyping of CMS Storage Management

2000-3 Carolien M.T. Metselaar (UvA)

Sociaal-organisatorische gevolgen van kennistechnologie; een

procesbenadering en actorperspectief.

2000-4 Geert de Haan (VU)

ETAG, A Formal Model of Competence Knowledge for User Interface Design

2000-5 Ruud van der Pol (UM)

Knowledge-based Query Formulation in Information Retrieval.

2000-6 Rogier van Eijk (UU)

Programming Languages for Agent Communication

2000-7 Niels Peek (UU)

Decision-theoretic Planning of Clinical Patient Management

2000-8 Veerle Coupé (EUR)

Sensitivity Analyis of Decision-Theoretic Networks

2000-9 Florian Waas (CWI)

Principles of Probabilistic Query Optimization

2000-10 Niels Nes (CWI)

Image Database Management System Design Considerations, Algorithms and Architecture

2000-11 Jonas Karlsson (CWI)

Scalable Distributed Data Structures for Database Management

2001

$===$

2001-1 Silja Renooij (UU)

Qualitative Approaches to Quantifying Probabilistic Networks

2001-2 Koen Hindriks (UU)

Agent Programming Languages: Programming with Mental Models

2001-3 Maarten van Someren (UvA)

Learning as problem solving

2001-4 Evgueni Smirnov (UM)

Conjunctive and Disjunctive Version Spaces with Instance-Based Boundary Sets 
2001-5 Jacco van Ossenbruggen (VU)

Processing Structured Hypermedia: A Matter of Style

2001-6 Martijn van Welie (VU)

Task-based User Interface Design

2001-7 Bastiaan Schonhage (VU)

Diva: Architectural Perspectives on Information Visualization

2001-8 Pascal van Eck (VU)

A Compositional Semantic Structure for Multi-Agent Systems Dynamics.

2001-9 Pieter Jan 't Hoen (RUL)

Towards Distributed Development of Large Object-Oriented Models, Views of Packages as Classes

2001-10 Maarten Sierhuis (UvA)

Modeling and Simulating Work Practice BRAHMS: a multiagent modeling and simulation language for work practice analysis and design

2001-11 Tom M. van Engers (VUA)

Knowledge Management: The Role of Mental Models in Business Systems Design

2002

$====$

2002-01 Nico Lassing (VU)

Architecture-Level Modifiability Analysis

2002-02 Roelof van Zwol (UT)

Modelling and searching web-based document collections

2002-03 Henk Ernst Blok (UT)

Database Optimization Aspects for Information Retrieval

2002-04 Juan Roberto Castelo Valdueza (UU)

The Discrete Acyclic Digraph Markov Model in Data Mining

2002-05 Radu Serban (VU)

The Private Cyberspace Modeling Electronic Environments inhabited by

Privacy-concerned Agents

2002-06 Laurens Mommers (UL)

Applied legal epistemology; Building a knowledge-based ontology of the legal domain

2002-07 Peter Boncz (CWI)

Monet: A Next-Generation DBMS Kernel For Query-Intensive Applications

2002-08 Jaap Gordijn (VU)

Value Based Requirements Engineering: Exploring Innovative E-Commerce Ideas

2002-09 Willem-Jan van den Heuvel (KUB)

Integrating Modern Business Applications with Objectified Legacy Systems

2002-10 Brian Sheppard (UM)

Towards Perfect Play of Scrabble 
2002-11 Wouter C.A. Wijngaards (VU)

Agent Based Modelling of Dynamics: Biological and Organisational Applications

2002-12 Albrecht Schmidt (UvA)

Processing XML in Database Systems

2002-13 Hongjing Wu (TUE)

A Reference Architecture for Adaptive Hypermedia Applications

2002-14 Wieke de Vries (UU)

Agent Interaction: Abstract Approaches to Modelling, Programming and Verifying Multi-Agent Systems

2002-15 Rik Eshuis (UT)

Semantics and Verification of UML Activity Diagrams for Workflow Modelling

2002-16 Pieter van Langen (VU)

The Anatomy of Design: Foundations, Models and Applications

2002-17 Stefan Manegold (UvA)

Understanding, Modeling, and Improving Main-Memory Database

Performance

2003

$===$

2003-01 Heiner Stuckenschmidt (VU)

Ontology-Based Information Sharing in Weakly Structured Environments

2003-02 Jan Broersen (VU)

Modal Action Logics for Reasoning About Reactive Systems

2003-03 Martijn Schuemie (TUD)

Human-Computer Interaction and Presence in Virtual Reality Exposure

Therapy

2003-04 Milan Petkovic (UT)

Content-Based Video Retrieval Supported by Database Technology

2003-05 Jos Lehmann (UvA)

Causation in Artificial Intelligence and Law - A modelling approach

2003-06 Boris van Schooten (UT)

Development and specification of virtual environments

2003-07 Machiel Jansen (UvA)

Formal Explorations of Knowledge Intensive Tasks

2003-08 Yongping Ran (UM)

Repair Based Scheduling

2003-09 Rens Kortmann (UM)

The resolution of visually guided behaviour

2003-10 Andreas Lincke (UvT)

Electronic Business Negotiation: Some experimental studies on the interaction between medium, innovation context and culture 
2003-11 Simon Keizer (UT)

Reasoning under Uncertainty in Natural Language Dialogue using Bayesian

Networks

2003-12 Roeland Ordelman (UT)

Dutch speech recognition in multimedia information retrieval

2003-13 Jeroen Donkers (UM)

Nosce Hostem - Searching with Opponent Models

2003-14 Stijn Hoppenbrouwers (KUN)

Freezing Language: Conceptualisation Processes across ICT-Supported

Organisations

2003-15 Mathijs de Weerdt (TUD)

Plan Merging in Multi-Agent Systems

2003-16 Menzo Windhouwer (CWI)

Feature Grammar Systems - Incremental Maintenance of Indexes to Digital

Media Warehouses

2003-17 David Jansen (UT)

Extensions of Statecharts with Probability, Time, and Stochastic Timing

2003-18 Levente Kocsis (UM)

Learning Search Decisions

2004

2004-01 Virginia Dignum (UU)

A Model for Organizational Interaction: Based on Agents, Founded in Logic

2004-02 Lai Xu (UvT)

Monitoring Multi-party Contracts for E-business

2004-03 Perry Groot (VU)

A Theoretical and Empirical Analysis of Approximation in Symbolic Problem

Solving

2004-04 Chris van Aart (UvA)

Organizational Principles for Multi-Agent Architectures

2004-05 Viara Popova (EUR)

Knowledge discovery and monotonicity

2004-06 Bart-Jan Hommes (TUD)

The Evaluation of Business Process Modeling Techniques

2004-07 Elise Boltjes (UM)

Voorbeeldig onderwijs; voorbeeldgestuurd onderwijs, een opstap naar abstract denken, vooral voor meisjes

2004-08 Joop Verbeek (UM)

Politie en de Nieuwe Internationale Informatiemarkt, Grensregionale politile gegevensuitwisseling en digitale expertise

2004-09 Martin Caminada (VU)

For the Sake of the Argument; explorations into argument-based reasoning

2004-10 Suzanne Kabel (UvA)

Knowledge-rich indexing of learning-objects 
2004-11 Michel Klein (VU)

Change Management for Distributed Ontologies

2004-12 The Duy Bui (UT)

Creating emotions and facial expressions for embodied agents

2004-13 Wojciech Jamroga (UT)

Using Multiple Models of Reality: On Agents who Know how to Play

2004-14 Paul Harrenstein (UU)

Logic in Conflict. Logical Explorations in Strategic Equilibrium

2004-15 Arno Knobbe (UU)

Multi-Relational Data Mining

2004-16 Federico Divina (VU)

Hybrid Genetic Relational Search for Inductive Learning

2004-17 Mark Winands (UM)

Informed Search in Complex Games

2004-18 Vania Bessa Machado (UvA)

Supporting the Construction of Qualitative Knowledge Models

2004-19 Thijs Westerveld (UT)

Using generative probabilistic models for multimedia retrieval

2004-20 Madelon Evers (Nyenrode)

Learning from Design: facilitating multidisciplinary design teams

2005

$===$

2005-01 Floor Verdenius (UvA)

Methodological Aspects of Designing Induction-Based Applications

2005-02 Erik van der Werf (UM))

AI techniques for the game of Go

2005-03 Franc Grootjen (RUN)

A Pragmatic Approach to the Conceptualisation of Language

2005-04 Nirvana Meratnia (UT)

Towards Database Support for Moving Object data

2005-05 Gabriel Infante-Lopez (UvA)

Two-Level Probabilistic Grammars for Natural Language Parsing

2005-06 Pieter Spronck (UM)

Adaptive Game AI

2005-07 Flavius Frasincar (TUE)

Hypermedia Presentation Generation for Semantic Web Information Systems

2005-08 Richard Vdovjak (TUE)

A Model-driven Approach for Building Distributed Ontology-based Web

Applications

2005-09 Jeen Broekstra (VU)

Storage, Querying and Inferencing for Semantic Web Languages

2005-10 Anders Bouwer (UvA)

Explaining Behaviour: Using Qualitative Simulation in Interactive Learning

Environments 
2005-11 Elth Ogston (VU)

Agent Based Matchmaking and Clustering - A Decentralized Approach to Search

2005-12 Csaba Boer (EUR)

Distributed Simulation in Industry

2005-13 Fred Hamburg (UL)

Een Computermodel voor het Ondersteunen van Euthanasiebeslissingen

2005-14 Borys Omelayenko (VU)

Web-Service configuration on the Semantic Web; Exploring how semantics meets pragmatics

2005-15 Tibor Bosse (VU)

Analysis of the Dynamics of Cognitive Processes

2005-16 Joris Graaumans (UU)

Usability of XML Query Languages

2005-17 Boris Shishkov (TUD)

Software Specification Based on Re-usable Business Components

2005-18 Danielle Sent (UU)

Test-selection strategies for probabilistic networks

2005-19 Michel van Dartel (UM)

Situated Representation

2005-20 Cristina Coteanu (UL)

Cyber Consumer Law, State of the Art and Perspectives

2005-21 Wijnand Derks (UT)

Improving Concurrency and Recovery in Database Systems by Exploiting Application Semantics

\footnotetext{
2006

$====$

2006-01 Samuil Angelov (TUE)

Foundations of B2B Electronic Contracting

2006-02 Cristina Chisalita (VU)

Contextual issues in the design and use of information technology in organizations

2006-03 Noor Christoph (UvA)

The role of metacognitive skills in learning to solve problems

2006-04 Marta Sabou (VU)

Building Web Service Ontologies

2006-05 Cees Pierik (UU)

Validation Techniques for Object-Oriented Proof Outlines

2006-06 Ziv Baida (VU)

Software-aided Service Bundling - Intelligent Methods \& Tools for Graphical Service Modeling

2006-07 Marko Smiljanic (UT)

XML schema matching - balancing efficiency and effectiveness by means of clustering
} 
2006-08 Eelco Herder (UT)

Forward, Back and Home Again - Analyzing User Behavior on the Web

2006-09 Mohamed Wahdan (UM)

Automatic Formulation of the Auditor's Opinion

2006-10 Ronny Siebes (VU)

Semantic Routing in Peer-to-Peer Systems

2006-11 Joeri van Ruth (UT)

Flattening Queries over Nested Data Types

2006-12 Bert Bongers (VU)

Interactivation - Towards an e-cology of people, our technological

environment, and the arts

2006-13 Henk-Jan Lebbink (UU)

Dialogue and Decision Games for Information Exchanging Agents

2006-14 Johan Hoorn (VU)

Software Requirements: Update, Upgrade, Redesign - towards a Theory of

Requirements Change

2006-15 Rainer Malik (UU)

CONAN: Text Mining in the Biomedical Domain

2006-16 Carsten Riggelsen (UU)

Approximation Methods for Efficient Learning of Bayesian Networks

2006-17 Stacey Nagata (UU)

User Assistance for Multitasking with Interruptions on a Mobile Device

2006-18 Valentin Zhizhkun (UvA)

Graph transformation for Natural Language Processing

2006-19 Birna van Riemsdijk (UU)

Cognitive Agent Programming: A Semantic Approach

2006-20 Marina Velikova (UvT)

Monotone models for prediction in data mining

2006-21 Bas van Gils (RUN)

Aptness on the Web

2006-22 Paul de Vrieze (RUN)

Fundaments of Adaptive Personalisation

2006-23 Ion Juvina (UU)

Development of Cognitive Model for Navigating on the Web

2006-24 Laura Hollink (VU)

Semantic Annotation for Retrieval of Visual Resources

2006-25 Madalina Drugan (UU)

Conditional log-likelihood MDL and Evolutionary MCMC

2006-26 Vojkan Mihajlovic (UT)

Score Region Algebra: A Flexible Framework for Structured Information

Retrieval

2006-27 Stefano Bocconi (CWI)

Vox Populi: generating video documentaries from semantically annotated media repositories

2006-28 Borkur Sigurbjornsson (UvA)

Focused Information Access using XML Element Retrieval 
2007-01 Kees Leune (UvT)

Access Control and Service-Oriented Architectures

2007-02 Wouter Teepe (RUG)

Reconciling Information Exchange and Confidentiality: A Formal Approach

2007-03 Peter Mika (VU)

Social Networks and the Semantic Web

2007-04 Jurriaan van Diggelen (UU)

Achieving Semantic Interoperability in Multi-agent Systems: a

dialogue-based approach

2007-05 Bart Schermer (UL)

Software Agents, Surveillance, and the Right to Privacy: a Legislative

Framework for Agent-enabled Surveillance

2007-06 Gilad Mishne (UvA)

Applied Text Analytics for Blogs

2007-07 Natasa Jovanovic' (UT)

To Whom It May Concern - Addressee Identification in Face-to-Face

Meetings

2007-08 Mark Hoogendoorn (VU)

Modeling of Change in Multi-Agent Organizations

2007-09 David Mobach (VU)

Agent-Based Mediated Service Negotiation

2007-10 Huib Aldewereld (UU)

Autonomy vs. Conformity: an Institutional Perspective on Norms and Protocols

2007-11 Natalia Stash (TUE)

Incorporating Cognitive/Learning Styles in a General-Purpose Adaptive

Hypermedia System

2007-12 Marcel van Gerven (RUN)

Bayesian Networks for Clinical Decision Support: A Rational Approach to

Dynamic Decision-Making under Uncertainty

2007-13 Rutger Rienks (UT)

Meetings in Smart Environments; Implications of Progressing Technology

2007-14 Niek Bergboer (UM)

Context-Based Image Analysis

2007-15 Joyca Lacroix (UM)

NIM: a Situated Computational Memory Model

2007-16 Davide Grossi (UU)

Designing Invisible Handcuffs. Formal investigations in Institutions and

Organizations for Multi-agent Systems

2007-17 Theodore Charitos (UU)

Reasoning with Dynamic Networks in Practice

2007-18 Bart Orriens (UvT)

On the development an management of adaptive business collaborations 
2007-19 David Levy (UM)

Intimate relationships with artificial partners

2007-20 Slinger Jansen (UU)

Customer Configuration Updating in a Software Supply Network

2007-21 Karianne Vermaas (UU)

Fast diffusion and broadening use: A research on residential adoption and usage of broadband internet in the Netherlands between 2001 and 2005

2007-22 Zlatko Zlatev (UT)

Goal-oriented design of value and process models from patterns

2007-23 Peter Barna (TUE)

Specification of Application Logic in Web Information Systems

2007-24 Georgina Ramírez Camps (CWI)

Structural Features in XML Retrieval

2007-25 Joost Schalken (VU)

Empirical Investigations in Software Process Improvement

2008

2008-01 Katalin Boer-Sorbán (EUR)

Agent-Based Simulation of Financial Markets: A modular, continuous-time approach

2008-02 Alexei Sharpanskykh (VU)

On Computer-Aided Methods for Modeling and Analysis of Organizations

2008-03 Vera Hollink (UvA)

Optimizing hierarchical menus: a usage-based approach

2008-04 Ander de Keijzer (UT)

Management of Uncertain Data - towards unattended integration

2008-05 Bela Mutschler (UT)

Modeling and simulating causal dependencies on process-aware information systems from a cost perspective

2008-06 Arjen Hommersom (RUN)

On the Application of Formal Methods to Clinical Guidelines, an Artificial Intelligence Perspective

2008-07 Peter van Rosmalen (OU)

Supporting the tutor in the design and support of adaptive e-learning

2008-08 Janneke Bolt (UU)

Bayesian Networks: Aspects of Approximate Inference

2008-09 Christof van Nimwegen (UU)

The paradox of the guided user: assistance can be counter-effective

2008-10 Wauter Bosma (UT)

Discourse oriented summarization

2008-11 Vera Kartseva (VU)

Designing Controls for Network Organizations: A Value-Based Approach

2008-12 Jozsef Farkas (RUN)

A Semiotically Oriented Cognitive Model of Knowledge Representation 
2008-13 Caterina Carraciolo (UvA)

Topic Driven Access to Scientific Handbooks

2008-14 Arthur van Bunningen (UT)

Context-Aware Querying; Better Answers with Less Effort

2008-15 Martijn van Otterlo (UT)

The Logic of Adaptive Behavior: Knowledge Representation and Algorithms

for the Markov Decision Process Framework in First-Order Domains.

2008-16 Henriette van Vugt (VU)

Embodied agents from a user's perspective

2008-17 Martin Op 't Land (TUD)

Applying Architecture and Ontology to the Splitting and Allying of

Enterprises

2008-18 Guido de Croon (UM)

Adaptive Active Vision

2008-19 Henning Rode (UT)

From Document to Entity Retrieval: Improving Precision and Performance of Focused Text Search

2008-20 Rex Arendsen (UvA)

Geen bericht, goed bericht. Een onderzoek naar de effecten van de introductie van elektronisch berichtenverkeer met de overheid op de administratieve lasten van bedrijven.

2008-21 Krisztian Balog (UvA)

People Search in the Enterprise

2008-22 Henk Koning (UU)

Communication of IT-Architecture

2008-23 Stefan Visscher (UU)

Bayesian network models for the management of ventilator-associated pneumonia

2008-24 Zharko Aleksovski (VU)

Using background knowledge in ontology matching

2008-25 Geert Jonker (UU)

Efficient and Equitable Exchange in Air Traffic Management Plan Repair using Spender-signed Currency

2008-26 Marijn Huijbregts (UT)

Segmentation, Diarization and Speech Transcription: Surprise Data Unraveled

2008-27 Hubert Vogten (OU)

Design and Implementation Strategies for IMS Learning Design

2008-28 Ildiko Flesch (RUN)

On the Use of Independence Relations in Bayesian Networks

2008-29 Dennis Reidsma (UT)

Annotations and Subjective Machines - Of Annotators, Embodied Agents,

Users, and Other Humans

2008-30 Wouter van Atteveldt (VU)

Semantic Network Analysis: Techniques for Extracting, Representing and Querying Media Content

2008-31 Loes Braun (UM)

Pro-Active Medical Information Retrieval 
2008-32 Trung H. Bui (UT)

Toward Affective Dialogue Management using Partially Observable Markov Decision Processes

2008-33 Frank Terpstra (UvA)

Scientific Workflow Design; theoretical and practical issues

2008-34 Jeroen de Knijf (UU)

Studies in Frequent Tree Mining

2008-35 Ben Torben Nielsen (UvT)

Dendritic morphologies: function shapes structure

2009

$===$

2009-01 Rasa Jurgelenaite (RUN)

Symmetric Causal Independence Models

2009-02 Willem Robert van Hage (VU)

Evaluating Ontology-Alignment Techniques

2009-03 Hans Stol (UvT)

A Framework for Evidence-based Policy Making Using IT

2009-04 Josephine Nabukenya (RUN)

Improving the Quality of Organisational Policy Making using Collaboration Engineering

2009-05 Sietse Overbeek (RUN)

Bridging Supply and Demand for Knowledge Intensive Tasks - Based on

Knowledge, Cognition, and Quality

2009-06 Muhammad Subianto (UU)

Understanding Classification

2009-07 Ronald Poppe (UT)

Discriminative Vision-Based Recovery and Recognition of Human Motion

2009-08 Volker Nannen (VU)

Evolutionary Agent-Based Policy Analysis in Dynamic Environments

2009-09 Benjamin Kanagwa (RUN)

Design, Discovery and Construction of Service-oriented Systems

2009-10 Jan Wielemaker (UvA)

Logic programming for knowledge-intensive interactive applications

2009-11 Alexander Boer (UvA)

Legal Theory, Sources of Law \& the Semantic Web

2009-12 Peter Massuthe (TUE, Humboldt-Universitaet zu Berlin)

Operating Guidelines for Services

2009-13 Steven de Jong (UM)

Fairness in Multi-Agent Systems

2009-14 Maksym Korotkiy (VU)

From ontology-enabled services to service-enabled ontologies (making ontologies work in e-science with ONTO-SOA)

2009-15 Rinke Hoekstra (UvA)

Ontology Representation - Design Patterns and Ontologies that Make Sense 
2009-16 Fritz Reul (UvT)

New Architectures in Computer Chess

2009-17 Laurens van der Maaten (UvT)

Feature Extraction from Visual Data

2009-18 Fabian Groffen (CWI)

Armada, An Evolving Database System

2009-19 Valentin Robu (CWI)

Modeling Preferences, Strategic Reasoning and Collaboration in

Agent-Mediated Electronic Markets

2009-20 Bob van der Vecht (UU)

Adjustable Autonomy: Controling Influences on Decision Making

2009-21 Stijn Vanderlooy (UM)

Ranking and Reliable Classification

2009-22 Pavel Serdyukov (UT)

Search For Expertise: Going beyond direct evidence

2009-23 Peter Hofgesang (VU)

Modelling Web Usage in a Changing Environment

2009-24 Annerieke Heuvelink (VUA)

Cognitive Models for Training Simulations

2009-25 Alex van Ballegooij (CWI)

RAM: Array Database Management through Relational Mapping

2009-26 Fernando Koch (UU)

An Agent-Based Model for the Development of Intelligent Mobile Services

2009-27 Christian Glahn (OU)

Contextual Support of social Engagement and Reflection on the Web

2009-28 Sander Evers (UT)

Sensor Data Management with Probabilistic Models

2009-29 Stanislav Pokraev (UT)

Model-Driven Semantic Integration of Service-Oriented Applications

2009-30 Marcin Zukowski (CWI)

Balancing vectorized query execution with bandwidth-optimized storage

2009-31 Sofiya Katrenko (UvA)

A Closer Look at Learning Relations from Text

2009-32 Rik Farenhorst (VU) and Remco de Boer (VU)

Architectural Knowledge Management: Supporting Architects and Auditors

2009-33 Khiet Truong (UT)

How Does Real Affect Affect Affect Recognition In Speech?

2009-34 Inge van de Weerd (UU)

Advancing in Software Product Management: An Incremental Method

Engineering Approach

2009-35 Wouter Koelewijn (UL)

Privacy en Politiegegevens; Over geautomatiseerde normatieve

informatie-uitwisseling

2009-36 Marco Kalz (OUN)

Placement Support for Learners in Learning Networks

2009-37 Hendrik Drachsler (OUN)

Navigation Support for Learners in Informal Learning Networks 
2009-38 Riina Vuorikari (OU)

Tags and self-organisation: a metadata ecology for learning resources in a multilingual context

2009-39 Christian Stahl (TUE, Humboldt-Universitaet zu Berlin)

Service Substitution - A Behavioral Approach Based on Petri Nets

2009-40 Stephan Raaijmakers (UvT)

Multinomial Language Learning: Investigations into the Geometry of

Language

2009-41 Igor Berezhnyy (UvT)

Digital Analysis of Paintings

2009-42 Toine Bogers (UvT)

Recommender Systems for Social Bookmarking

2009-43 Virginia Nunes Leal Franqueira (UT)

Finding Multi-step Attacks in Computer Networks using Heuristic Search and Mobile Ambients

2009-44 Roberto Santana Tapia (UT)

Assessing Business-IT Alignment in Networked Organizations

2009-45 Jilles Vreeken (UU)

Making Pattern Mining Useful

2009-46 Loredana Afanasiev (UvA)

Querying XML: Benchmarks and Recursion

$===$
2010

$===$

2010-01 Matthijs van Leeuwen (UU)

Patterns that Matter

2010-02 Ingo Wassink (UT)

Work flows in Life Science

2010-03 Joost Geurts (CWI)

A Document Engineering Model and Processing Framework for Multimedia documents

2010-04 Olga Kulyk (UT)

Do You Know What I Know? Situational Awareness of Co-located Teams in Multidisplay Environments

2010-05 Claudia Hauff (UT)

Predicting the Effectiveness of Queries and Retrieval Systems

2010-06 Sander Bakkes (UvT)

Rapid Adaptation of Video Game AI

2010-07 Wim Fikkert (UT)

Gesture interaction at a Distance

2010-08 Krzysztof Siewicz (UL)

Towards an Improved Regulatory Framework of Free Software. Protecting user freedoms in a world of software communities and eGovernments

2010-09 Hugo Kielman (UL)

A Politiele gegevensverwerking en Privacy, Naar een effectieve waarborging 
2010-10 Rebecca Ong (UL)

Mobile Communication and Protection of Children

2010-11 Adriaan Ter Mors (TUD)

The world according to MARP: Multi-Agent Route Planning

2010-12 Susan van den Braak (UU)

Sensemaking software for crime analysis

2010-13 Gianluigi Folino (RUN)

High Performance Data Mining using Bio-inspired techniques

2010-14 Sander van Splunter (VU)

Automated Web Service Reconfiguration

2010-15 Lianne Bodenstaff (UT)

Managing Dependency Relations in Inter-Organizational Models

2010-16 Sicco Verwer (TUD)

Efficient Identification of Timed Automata, theory and practice

2010-17 Spyros Kotoulas (VU)

Scalable Discovery of Networked Resources: Algorithms, Infrastructure,

Applications

2010-18 Charlotte Gerritsen (VU)

Caught in the Act: Investigating Crime by Agent-Based Simulation

2010-19 Henriette Cramer (UvA)

People's Responses to Autonomous and Adaptive Systems

2010-20 Ivo Swartjes (UT)

Whose Story Is It Anyway? How Improv Informs Agency and Authorship of Emergent Narrative

2010-21 Harold van Heerde (UT)

Privacy-aware data management by means of data degradation

2010-22 Michiel Hildebrand (CWI)

End-user Support for Access to Heterogeneous Linked Data

2010-23 Bas Steunebrink (UU)

The Logical Structure of Emotions

2010-24 Dmytro Tykhonov

Designing Generic and Efficient Negotiation Strategies

2010-25 Zulfiqar Ali Memon (VU)

Modelling Human-Awareness for Ambient Agents: A Human Mindreading

Perspective

2010-26 Ying Zhang (CWI)

XRPC: Efficient Distributed Query Processing on Heterogeneous XQuery

Engines

2010-27 Marten Voulon (UL)

Automatisch contracteren

2010-28 Arne Koopman (UU)

Characteristic Relational Patterns

2010-29 Stratos Idreos (CWI)

Database Cracking: Towards Auto-tuning Database Kernels

2010-30 Marieke van Erp (UvT)

Accessing Natural History - Discoveries in data cleaning, structuring, and retrieval 
2010-31 Victor de Boer (UvA)

Ontology Enrichment from Heterogeneous Sources on the Web

2010-32 Marcel Hiel (UvT)

An Adaptive Service Oriented Architecture: Automatically solving Interoperability Problems

2010-33 Robin Aly (UT)

Modeling Representation Uncertainty in Concept-Based Multimedia Retrieval

2010-34 Teduh Dirgahayu (UT)

Interaction Design in Service Compositions

2010-35 Dolf Trieschnigg (UT)

Proof of Concept: Concept-based Biomedical Information Retrieval

2010-36 Jose Janssen (OU)

Paving the Way for Lifelong Learning; Facilitating competence development through a learning path specification

2010-37 Niels Lohmann (TUE)

Correctness of services and their composition

2010-38 Dirk Fahland (TUE)

From Scenarios to components

2010-39 Ghazanfar Farooq Siddiqui (VU)

Integrative modeling of emotions in virtual agents

2010-40 Mark van Assem (VU)

Converting and Integrating Vocabularies for the Semantic Web

2010-41 Guillaume Chaslot (UM)

Monte-Carlo Tree Search

2010-42 Sybren de Kinderen (VU)

Needs-driven service bundling in a multi-supplier setting - the computational e3-service approach

2010-43 Peter van Kranenburg (UU)

A Computational Approach to Content-Based Retrieval of Folk Song

Melodies

2010-44 Pieter Bellekens (TUE)

An Approach towards Context-sensitive and User-adapted Access to

Heterogeneous Data Sources, Illustrated in the Television Domain

2010-45 Vasilios Andrikopoulos (UvT)

A theory and model for the evolution of software services

2010-46 Vincent Pijpers (VU)

e3alignment: Exploring Inter-Organizational Business-ICT Alignment

2010-47 Chen Li (UT)

Mining Process Model Variants: Challenges, Techniques, Examples

2010-48 Withdrawn

2010-49 Jahn-Takeshi Saito (UM)

Solving difficult game positions

2010-50 Bouke Huurnink (UvA)

Search in Audiovisual Broadcast Archives

2010-51 Alia Khairia Amin (CWI)

Understanding and supporting information seeking tasks in multiple sources 
2010-52 Peter-Paul van Maanen (VU)

Adaptive Support for Human-Computer Teams: Exploring the Use of

Cognitive Models of Trust and Attention

2010-53 Edgar Meij (UvA)

Combining Concepts and Language Models for Information Access

2011

$===$

2011-01 Botond Cseke (RUN)

Variational Algorithms for Bayesian Inference in Latent Gaussian Models

2011-02 Nick Tinnemeier (UU)

Organizing Agent Organizations. Syntax and Operational Semantics of an

Organization-Oriented Programming Language

2011-03 Jan Martijn van der Werf (TUE)

Compositional Design and Verification of Component-Based Information

Systems

2011-04 Hado van Hasselt (UU)

Insights in Reinforcement Learning; Formal analysis and empirical evaluation of temporal-difference learning algorithms

2011-05 Base van der Raadt (VU)

Enterprise Architecture Coming of Age - Increasing the Performance of an Emerging Discipline.

2011-06 Yiwen Wang (TUE)

Semantically-Enhanced Recommendations in Cultural Heritage

2011-07 Yujia Cao (UT)

Multimodal Information Presentation for High Load Human Computer

Interaction

2011-08 Nieske Vergunst (UU)

BDI-based Generation of Robust Task-Oriented Dialogues

2011-09 Tim de Jong (OU)

Contextualised Mobile Media for Learning

2011-10 Bart Bogaert (UvT)

Cloud Content Contention

2011-11 Dhaval Vyas (UT)

Designing for Awareness: An Experience-focused HCI Perspective

2011-12 Carmen Bratosin (TUE)

Grid Architecture for Distributed Process Mining

2011-13 Xiaoyu Mao (UvT)

Airport under Control. Multiagent Scheduling for Airport Ground Handling

2011-14 Milan Lovric (EUR)

Behavioral Finance and Agent-Based Artificial Markets

2011-15 Marijn Koolen (UvA)

The Meaning of Structure: the Value of Link Evidence for Information

Retrieval 
2011-16 Maarten Schadd (UM)

Selective Search in Games of Different Complexity

2011-17 Jiyin He (UvA)

Exploring Topic Structure: Coherence, Diversity and Relatedness

2011-18 Mark Ponsen (UM)

Strategic Decision-Making in complex games

2011-19 Ellen Rusman (OU)

The Mind' s Eye on Personal Profiles

2011-20 Qing Gu (VU)

Guiding service-oriented software engineering - A view-based approach

2011-21 Linda Terlouw (TUD)

Modularization and Specification of Service-Oriented Systems

2011-22 Junte Zhang (UvA)

System Evaluation of Archival Description and Access

2011-23 Wouter Weerkamp (UvA)

Finding People and their Utterances in Social Media

2011-24 Herwin van Welbergen (UT)

Behavior Generation for Interpersonal Coordination with Virtual Humans On

Specifying, Scheduling and Realizing Multimodal Virtual Human Behavior

2011-25 Syed Waqar ul Qounain Jaffry (VU))

Analysis and Validation of Models for Trust Dynamics

2011-26 Matthijs Aart Pontier (VU)

Virtual Agents for Human Communication - Emotion Regulation and

Involvement-Distance Trade-Offs in Embodied Conversational Agents and Robots

2011-27 Aniel Bhulai (VU)

Dynamic website optimization through autonomous management of design patterns

2011-28 Rianne Kaptein (UvA)

Effective Focused Retrieval by Exploiting Query Context and Document

Structure

2011-29 Faisal Kamiran (TUE)

Discrimination-aware Classification

2011-30 Egon van den Broek (UT)

Affective Signal Processing (ASP): Unraveling the mystery of emotions

2011-31 Ludo Waltman (EUR)

Computational and Game-Theoretic Approaches for Modeling Bounded

Rationality

2011-32 Nees-Jan van Eck (EUR)

Methodological Advances in Bibliometric Mapping of Science

2011-33 Tom van der Weide (UU)

Arguing to Motivate Decisions

2011-34 Paolo Turrini (UU)

Strategic Reasoning in Interdependence: Logical and Game-theoretical

Investigations

2011-35 Maaike Harbers (UU)

Explaining Agent Behavior in Virtual Training 
2011-36 Erik van der Spek (UU)

Experiments in serious game design: a cognitive approach

2011-37 Adriana Burlutiu (RUN)

Machine Learning for Pairwise Data, Applications for Preference Learning and Supervised Network Inference

2011-38 Nyree Lemmens (UM)

Bee-inspired Distributed Optimization

2011-39 Joost Westra (UU)

Organizing Adaptation using Agents in Serious Games

2011-40 Viktor Clerc (VU)

Architectural Knowledge Management in Global Software Development

2011-41 Luan Ibraimi (UT)

Cryptographically Enforced Distributed Data Access Control

2011-42 Michal Sindlar (UU)

Explaining Behavior through Mental State Attribution

2011-43 Henk van der Schuur (UU)

Process Improvement through Software Operation Knowledge

2011-44 Boris Reuderink (UT)

Robust Brain-Computer Interfaces

2011-45 Herman Stehouwer (UvT)

Statistical Language Models for Alternative Sequence Selection

2011-46 Beibei Hu (TUD)

Towards Contextualized Information Delivery: A Rule-based Architecture for the Domain of Mobile Police Work

2011-47 Azizi Bin Ab Aziz (VU)

Exploring Computational Models for Intelligent Support of Persons with Depression

2011-48 Mark Ter Maat (UT)

Response Selection and Turn-taking for a Sensitive Artificial Listening Agent

2011-49 Andreea Niculescu (UT)

Conversational interfaces for task-oriented spoken dialogues: design aspects influencing interaction quality

2012

$===$

2012-01 Terry Kakeeto (UvT)

Relationship Marketing for SMEs in Uganda

2012-02 Muhammad Umair (VU)

Adaptivity, emotion, and Rationality in Human and Ambient Agent Models

2012-03 Adam Vanya (VU)

Supporting Architecture Evolution by Mining Software Repositories

2012-04 Jurriaan Souer (UU)

Development of Content Management System-based Web Applications

2012-05 Marijn Plomp (UU)

Maturing Interorganisational Information Systems 
2012-06 Wolfgang Reinhardt (OU)

Awareness Support for Knowledge Workers in Research Networks

2012-07 Rianne van Lambalgen (VU)

When the Going Gets Tough: Exploring Agent-based Models of Human

Performance under Demanding Conditions

2012-08 Gerben de Vries (UvA)

Kernel Methods for Vessel Trajectories

2012-09 Ricardo Neisse (UT)

Trust and Privacy Management Support for Context-Aware Service Platforms

2012-10 David Smits (TUE)

Towards a Generic Distributed Adaptive Hypermedia Environment

2012-11 J.C.B. Rantham Prabhakara (TUE)

Process Mining in the Large: Preprocessing, Discovery, and Diagnostics

2012-12 Kees van der Sluijs (TUE)

Model Driven Design and Data Integration in Semantic Web Information

Systems

2012-13 Suleman Shahid (UvT)

Fun and Face: Exploring non-verbal expressions of emotion during playful interactions

2012-14 Evgeny Knutov (TUE)

Generic Adaptation Framework for Unifying Adaptive Web-based Systems

2012-15 Natalie van der Wal (VU)

Social Agents. Agent-Based Modelling of Integrated Internal and Social

Dynamics of Cognitive and Affective Processes.

2012-16 Fiemke Both (VU)

Helping people by understanding them - Ambient Agents supporting task execution and depression treatment

2012-17 Amal Elgammal (UvT)

Towards a Comprehensive Framework for Business Process Compliance

2012-18 Eltjo Poort (VU)

Improving Solution Architecting Practices

2012-19 Helen Schonenberg (TUE)

What's Next? Operational Support for Business Process Execution

2012-20 Ali Bahramisharif (RUN)

Covert Visual Spatial Attention, a Robust Paradigm for Brain-Computer

Interfacing

2012-21 Roberto Cornacchia (TUD)

Querying Sparse Matrices for Information Retrieval

2012-22 Thijs Vis (UvT)

Intelligence, politie en veiligheidsdienst: verenigbare grootheden?

2012-23 Christian Muehl (UT)

Toward Affective Brain-Computer Interfaces: Exploring the Neurophysiology of Affect during Human Media Interaction

2012-24 Laurens van der Werff (UT)

Evaluation of Noisy Transcripts for Spoken Document Retrieval 
2012-25 Silja Eckartz (UT)

Managing the Business Case Development in Inter-Organizational IT

Projects: A Methodology and its Application

2012-26 Emile de Maat (UvA)

Making Sense of Legal Text

2012-27 Hayrettin Gurkok (UT)

Mind the Sheep! User Experience Evaluation \& Brain-Computer Interface

Games

2012-28 Nancy Pascall (UvT)

Engendering Technology Empowering Women

2012-29 Almer Tigelaar (UT)

Peer-to-Peer Information Retrieval

2012-30 Alina Pommeranz (TUD)

Designing Human-Centered Systems for Reflective Decision Making

2012-31 Emily Bagarukayo (RUN)

A Learning by Construction Approach for Higher Order Cognitive Skills

Improvement, Building Capacity and Infrastructure

2012-32 Wietske Visser (TUD)

Qualitative multi-criteria preference representation and reasoning

2012-33 Rory Sie (OUN)

Coalitions in Cooperation Networks (COCOON)

2012-34 Pavol Jancura (RUN)

Evolutionary analysis in PPI networks and applications

2012-35 Evert Haasdijk (VU)

Never Too Old To Learn - On-line Evolution of Controllers in Swarm- and

Modular Robotics

2012-36 Denis Ssebugwawo (RUN)

Analysis and Evaluation of Collaborative Modeling Processes

2012-37 Agnes Nakakawa (RUN)

A Collaboration Process for Enterprise Architecture Creation

2012-38 Selmar Smit (VU)

Parameter Tuning and Scientific Testing in Evolutionary Algorithms

2012-39 Hassan Fatemi (UT)

Risk-aware design of value and coordination networks

2012-40 Agus Gunawan (UvT)

Information Access for SMEs in Indonesia

2012-41 Sebastian Kelle (OU)

Game Design Patterns for Learning

2012-42 Dominique Verpoorten (OU)

Reflection Amplifiers in self-regulated Learning

2012-43 Withdrawn

2012-44 Anna Tordai (VU)

On Combining Alignment Techniques

2012-45 Benedikt Kratz (UvT)

A Model and Language for Business-aware Transactions 
2012-46 Simon Carter (UvA)

Exploration and Exploitation of Multilingual Data for Statistical Machine

Translation

2012-47 Manos Tsagkias (UvA)

Mining Social Media: Tracking Content and Predicting Behavior

2012-48 Jorn Bakker (TUE)

Handling Abrupt Changes in Evolving Time-series Data

2012-49 Michael Kaisers (UM)

Learning against Learning - Evolutionary dynamics of reinforcement learning algorithms in strategic interactions

2012-50 Steven van Kervel (TUD)

Ontologogy driven Enterprise Information Systems Engineering

2012-51 Jeroen de Jong (TUD)

Heuristics in Dynamic Sceduling; a practical framework with a case study in elevator dispatching

2013

$===$

2013-01 Viorel Milea (EUR)

News Analytics for Financial Decision Support

2013-02 Erietta Liarou (CWI)

MonetDB/DataCell: Leveraging the Column-store Database Technology for

Efficient and Scalable Stream Processing

2013-03 Szymon Klarman (VU)

Reasoning with Contexts in Description Logics

2013-04 Chetan Yadati (TUD)

Coordinating autonomous planning and scheduling

2013-05 Dulce Pumareja (UT)

Groupware Requirements Evolutions Patterns

2013-06 Romulo Goncalves (CWI)

The Data Cyclotron: Juggling Data and Queries for a Data Warehouse

Audience

2013-07 Giel van Lankveld (UvT)

Quantifying Individual Player Differences

2013-08 Robbert-Jan Merk (VU)

Making enemies: cognitive modeling for opponent agents in fighter pilot simulators

2013-09 Fabio Gori (RUN)

Metagenomic Data Analysis: Computational Methods and Applications

2013-10 Jeewanie Jayasinghe Arachchige (UvT)

A Unified Modeling Framework for Service Design.

2013-11 Evangelos Pournaras (TUD)

Multi-level Reconfigurable Self-organization in Overlay Services

2013-12 Marian Razavian (VU)

Knowledge-driven Migration to Services 
2013-13 Mohammad Safiri (UT)

Service Tailoring: User-centric creation of integrated IT-based homecare

services to support independent living of elderly

2013-14 Jafar Tanha (UvA)

Ensemble Approaches to Semi-Supervised Learning Learning

2013-15 Daniel Hennes (UM)

Multiagent Learning - Dynamic Games and Applications

2013-16 Eric Kok (UU)

Exploring the practical benefits of argumentation in multi-agent deliberation

2013-17 Koen Kok (VU)

The PowerMatcher: Smart Coordination for the Smart Electricity Grid

2013-18 Jeroen Janssens (UvT)

Outlier Selection and One-Class Classification

2013-19 Renze Steenhuizen (TUD)

Coordinated Multi-Agent Planning and Scheduling

2013-20 Katja Hofmann (UvA)

Fast and Reliable Online Learning to Rank for Information Retrieval

2013-21 Sander Wubben (UvT)

Text-to-text generation by monolingual machine translation

2013-22 Tom Claassen (RUN)

Causal Discovery and Logic

2013-23 Patricio de Alencar Silva (UvT)

Value Activity Monitoring

2013-24 Haitham Bou Ammar (UM)

Automated Transfer in Reinforcement Learning

2013-25 Agnieszka Anna Latoszek-Berendsen (UM)

Intention-based Decision Support. A new way of representing and implementing clinical guidelines in a Decision Support System

2013-26 Alireza Zarghami (UT)

Architectural Support for Dynamic Homecare Service Provisioning

2013-27 Mohammad Huq (UT)

Inference-based Framework Managing Data Provenance

2013-28 Frans van der Sluis (UT)

When Complexity becomes Interesting: An Inquiry into the Information eXperience

2013-29 Iwan de Kok (UT)

Listening Heads

2013-30 Joyce Nakatumba (TUE)

Resource-Aware Business Process Management: Analysis and Support

2013-31 Dinh Khoa Nguyen (UvT)

Blueprint Model and Language for Engineering Cloud Applications

2013-32 Kamakshi Rajagopal (OUN)

Networking For Learning; The role of Networking in a Lifelong Learner's

Professional Development

2013-33 Qi Gao (TUD)

User Modeling and Personalization in the Microblogging Sphere 
2013-34 Kien Tjin-Kam-Jet (UT)

Distributed Deep Web Search

2013-35 Abdallah El Ali (UvA)

Minimal Mobile Human Computer Interaction

2013-36 Than Lam Hoang (TUe)

Pattern Mining in Data Streams

2013-37 Dirk Börner (OUN)

Ambient Learning Displays

2013-38 Eelco den Heijer (VU)

Autonomous Evolutionary Art

2013-39 Joop de Jong (TUD)

A Method for Enterprise Ontology based Design of Enterprise Information

Systems

2013-40 Pim Nijssen (UM)

Monte-Carlo Tree Search for Multi-Player Games

2013-41 Jochem Liem (UvA)

Supporting the Conceptual Modelling of Dynamic Systems: A Knowledge

Engineering Perspective on Qualitative Reasoning

2013-42 Léon Planken (TUD)

Algorithms for Simple Temporal Reasoning

2013-43 Marc Bron (UvA)

Exploration and Contextualization through Interaction and Concepts

2014

$===$

2014-01 Nicola Barile (UU)

Studies in Learning Monotone Models from Data

2014-02 Fiona Tuliyano (RUN)

Combining System Dynamics with a Domain Modeling Method

2014-03 Sergio Raul Duarte Torres (UT)

Information Retrieval for Children: Search Behavior and Solutions

2014-04 Hanna Jochmann-Mannak (UT)

Websites for children: search strategies and interface design - Three studies on children's search performance and evaluation

2014-05 Jurriaan van Reijsen (UU)

Knowledge Perspectives on Advancing Dynamic Capability

2014-06 Damian Tamburri (VU)

Supporting Networked Software Development

2014-07 Arya Adriansyah (TUE)

Aligning Observed and Modeled Behavior

2014-08 Samur Araujo (TUD)

Data Integration over Distributed and Heterogeneous Data Endpoints

2014-09 Philip Jackson (UvT)

Toward Human-Level Artificial Intelligence: Representation and Computation of Meaning in Natural Language 
2014-10 Ivan Salvador Razo Zapata (VU)

Service Value Networks

2014-11 Janneke van der Zwaan (TUD)

An Empathic Virtual Buddy for Social Support

2014-12 Willem van Willigen (VU)

Look Ma, No Hands: Aspects of Autonomous Vehicle Control

2014-13 Arlette van Wissen (VU)

Agent-Based Support for Behavior Change: Models and Applications in

Health and Safety Domains

2014-14 Yangyang Shi (TUD)

Language Models With Meta-information

2014-15 Natalya Mogles (VU)

Agent-Based Analysis and Support of Human Functioning in Complex

Socio-Technical Systems: Applications in Safety and Healthcare

2014-16 Krystyna Milian (VU)

Supporting trial recruitment and design by automatically interpreting

eligibility criteria

2014-17 Kathrin Dentler (VU)

Computing healthcare quality indicators automatically: Secondary Use of

Patient Data and Semantic Interoperability

2014-18 Mattijs Ghijsen (VU)

Methods and Models for the Design and Study of Dynamic Agent

Organizations

2014-19 Vinicius Ramos (TUE)

Adaptive Hypermedia Courses: Qualitative and Quantitative Evaluation and

Tool Support

2014-20 Mena Habib (UT)

Named Entity Extraction and Disambiguation for Informal Text: The Missing

Link

2014-21 Kassidy Clark (TUD)

Negotiation and Monitoring in Open Environments

2014-22 Marieke Peeters (UU)

Personalized Educational Games - Developing agent-supported

scenario-based training

2014-23 Eleftherios Sidirourgos (UvA/CWI)

Space Efficient Indexes for the Big Data Era

2014-24 Davide Ceolin (VU)

Trusting Semi-structured Web Data

2014-25 Martijn Lappenschaar (RUN)

New network models for the analysis of disease interaction

2014-26 Tim Baarslag (TUD)

What to Bid and When to Stop

2014-27 Rui Jorge Almeida (EUR)

Conditional Density Models Integrating Fuzzy and Probabilistic

Representations of Uncertainty

2014-28 Anna Chmielowiec (VU)

Decentralized k-Clique Matching 
2014-29 Jaap Kabbedijk (UU)

Variability in Multi-Tenant Enterprise Software

2014-30 Peter de Cock (UvT)

Anticipating Criminal Behaviour

2014-31 Leo van Moergestel (UU)

Agent Technology in Agile Multiparallel Manufacturing and Product Support

2014-32 Naser Ayat (UvA)

On Entity Resolution in Probabilistic Data

2014-33 Tesfa Tegegne (RUN)

Service Discovery in eHealth

2014-34 Christina Manteli (VU)

The Effect of Governance in Global Software Development: Analyzing

Transactive Memory Systems.

2014-35 Joost van Ooijen (UU)

Cognitive Agents in Virtual Worlds: A Middleware Design Approach

2014-36 Joos Buijs (TUE)

Flexible Evolutionary Algorithms for Mining Structured Process Models

2014-37 Maral Dadvar (UT)

Experts and Machines United Against Cyberbullying

2014-38 Danny Plass-Oude Bos (UT)

Making brain-computer interfaces better: improving usability through post-processing.

2014-39 Jasmina Maric (UvT)

Web Communities, Immigration, and Social Capital

2014-40 Walter Omona (RUN)

A Framework for Knowledge Management Using ICT in Higher Education

2014-41 Frederic Hogenboom (EUR)

Automated Detection of Financial Events in News Text

2014-42 Carsten Eijckhof (CWI/TUD)

Contextual Multidimensional Relevance Models

2014-43 Kevin Vlaanderen (UU)

Supporting Process Improvement using Method Increments

2014-44 Paulien Meesters (UvT)

Intelligent Blauw. Intelligence-gestuurde politiezorg in gebiedsgebonden eenheden.

2014-45 Birgit Schmitz (OUN)

Mobile Games for Learning: A Pattern-Based Approach

2014-46 Ke Tao (TUD)

Social Web Data Analytics: Relevance, Redundancy, Diversity

2014-47 Shangsong Liang (UvA)

Fusion and Diversification in Information Retrieval

2015

$===$ 
2015-01 Niels Netten (UvA)

Machine Learning for Relevance of Information in Crisis Response

2015-02 Faiza Bukhsh (UvT)

Smart auditing: Innovative Compliance Checking in Customs Controls

2015-03 Twan van Laarhoven (RUN)

Machine learning for network data

2015-04 Howard Spoelstra (OUN)

Collaborations in Open Learning Environments

2015-05 Christoph Bösch (UT)

Cryptographically Enforced Search Pattern Hiding

2015-06 Farideh Heidari (TUD)

Business Process Quality Computation - Computing Non-Functional

Requirements to Improve Business Processes

2015-07 Maria-Hendrike Peetz (UvA)

Time-Aware Online Reputation Analysis

2015-08 Jie Jiang (TUD)

Organizational Compliance: An agent-based model for designing and evaluating organizational interactions

2015-09 Randy Klaassen (UT)

HCI Perspectives on Behavior Change Support Systems

2015-10 Henry Hermans (OUN)

OpenU: design of an integrated system to support lifelong learning

2015-11 Yongming Luo (TUE)

Designing algorithms for big graph datasets: A study of computing

bisimulation and joins

2015-12 Julie M. Birkholz (VU)

Modi Operandi of Social Network Dynamics: The Effect of Context on

Scientific Collaboration Networks

2015-13 Giuseppe Procaccianti (VU)

Energy-Efficient Software

2015-14 Bart van Straalen (UT)

A cognitive approach to modeling bad news conversations

2015-15 Klaas Andries de Graaf (VU)

Ontology-based Software Architecture Documentation

2015-16 Changyun Wei (TUD)

Cognitive Coordination for Cooperative Multi-Robot Teamwork

2015-17 André van Cleeff (UT)

Physical and Digital Security Mechanisms: Properties, Combinations and Trade-offs 


\section{Samenvatting}

Automatisering impliceert de vervanging van fysieke door digitale systemen: we gebruiken informatietechnologie immers omdat sommige eigenschappen van software, zoals hoge snelheid, lage kosten en accuratesse wenselijker zijn dan de eigenschappen van fysieke systemen.

Helaas zijn digitale systemen niet altijd beter dan fysieke systemen. Specifiek kan automatisering een negatief effect hebben op de beschikbaarheid, integriteit en vertrouwelijkheid van informatie. Specifiek vergemakkelijkt het Internet de verspreiding van informatie en het daarmee wordt het moeilijker om informatie vertrouwelijk te houden. De toename in connectiviteit veroorzaakt door het Internet leidt ertoe dat organisaties"gedeperimeteriseerd" worden, de fysieke barrières die ze voorheen afschermden van de omgeving worden afgebroken.

We constateren niettemin dat er geen uitgebreid en gestructureerd geheel van kennis is over de verschillen tussen fysieke en digitale systemen en de wijze waarop deperimeterisatie plaatsvindt. Het verkrijgen van deze kennis wordt belangrijker nu fysieke en digitale systemen samensmelten in het Internet of Things: alleen wanneer we de verschillen tussen fysieke en digitale systemen begrijpen kunnen we systemen als smart buildings met camera's en op afstand bestuurbare deuren werkelijk veilig realiseren.

Het ontwikkelen van deze kennis begint met een simpel conceptueel raamwerk: systemen variëren van compleet fysiek tot compleet digital. Fysieke systemen gebruiken alleen fysieke beveiligingsmechanismen, en digitale systemen gebruiken alleen digitale beveiligingsmechanismen. Hier tussenin is een categorie gemengde of hybride systemen, die zowel digitale als fysieke beveiligingsmechanismen gebruiken. Met behulp van dit raamwerk onderzoeken we de beveiliging van fysieke, digitale en hybride systemen in vier domeinen: toegangsbeheer, stemmen in verkiezingen, IT infrastructuur en copyright.

We beginnen met een onderzoek naar de onderliggende eigenschappen van fysieke en digitale systemen: karakteristieken van een fysiek of digitaal object die, onder specifieke condities, positieve of negatieve effecten hebben op de beveiliging. In totaal identificeren we twintig fysieke en vijf digitale beveiligingseigenschappen. Hiermee worden de verschillen tussen fysieke en digitale systemen geïdentificeerd. 
Vervolgens onderzoeken we hybride systemen om te begrijpen hoe fysieke en digitale beveiligingsmechanismen gecombineerd kunnen worden, en wat de tradeoffs zijn tussen deze twee. Tot slot gebruiken we deze resultaten om twee methoden te ontwikkelen die gebruikt kunnen worden om systemen beter te beveiligen:

- Een methode voor het inschatten van informatiebeveiligingsrisico's van fysieke, digitale en hybride systemen. Deze methode is gebaseerd op de genoemde beveiligingseigenschappen: ze worden gebruikt om de beveiliging van bestaande systemen te begrijpen (door de eigenschappen te identificeren en hoe deze zouden kunnen veranderen) of om nieuwe systemen te ontwerpen (door de eigenschappen in te bouwen die een positief effect hebben op informatiebeveiliging).

- Een methode voor het ontwerpen en analyseren van hybride systemen met behulp van patterns. Deze herbruikbare ontwerpen laten zien hoe fysieke en digitale beveiliging optimaal gecombineerd kunnen worden. In totaal presenteren we dertien patterns die zowel bruikbaar zijn voor het ontwerpen als voor het evalueren van hybride systemen.

Beide methoden zijn met succes getest in een focus group meeting met beveiligingsexperts. 

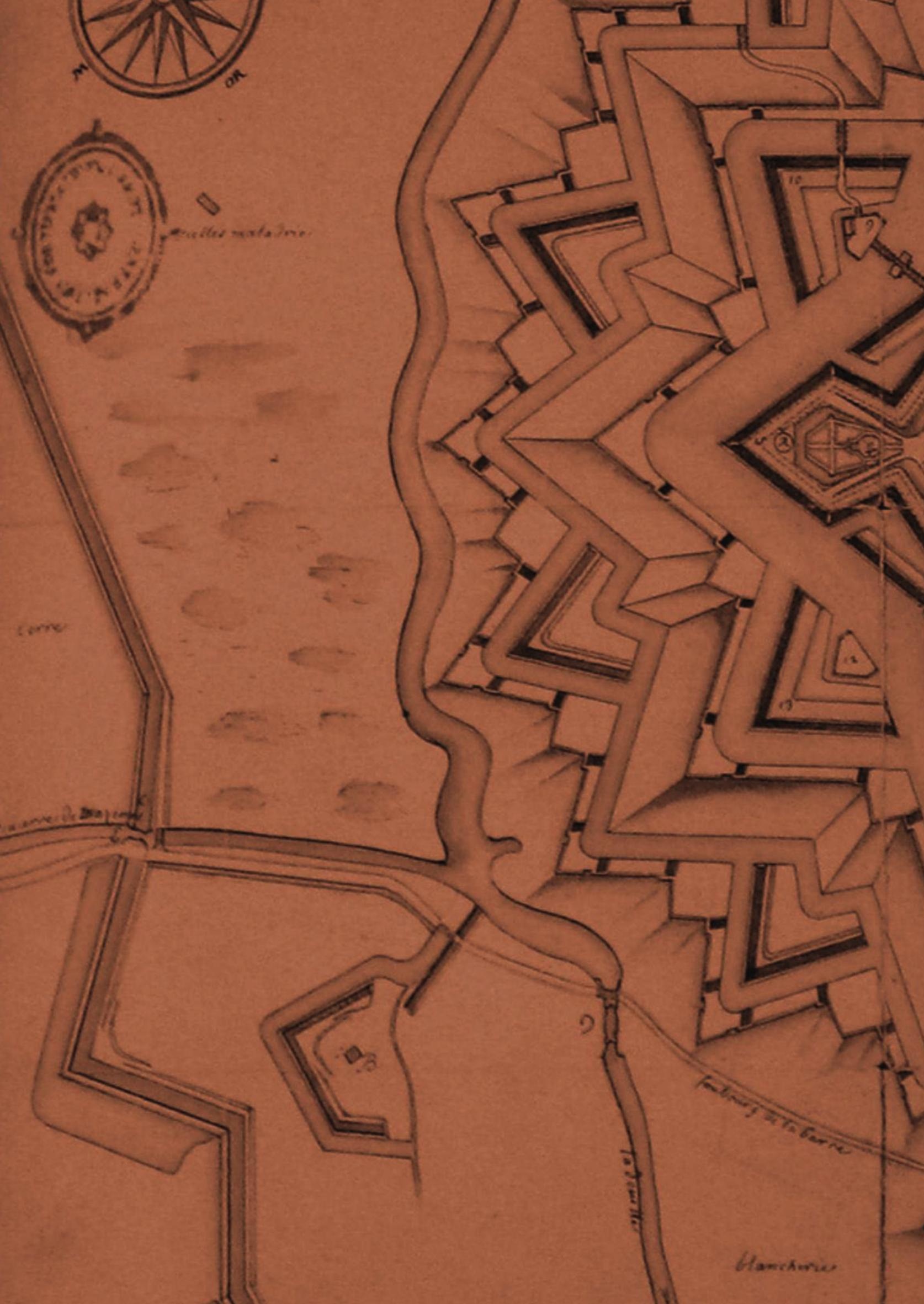